\title{
Trade-offs between multiple ecosystem services in UK and US salt marshes
}

\author{
Kate Davidson \\ Department of Biosciences
}

Swansea University

2019

Submitted to Swansea University in fulfilment of the requirements for the Degree of Doctor of Philosophy

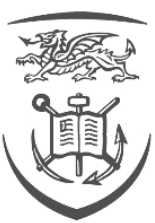

Prifysgol Abertawe Swansea University 


\section{Summary}

Salt marshes supply vital ecosystem services (ES), providing material goods and recreation space, regulating natural hazards, and supporting diverse wildlife. However, increases in the utilisation of one ES can lead to reductions or 'trade-offs' in others. Because salt marshes are commonly used for grazing livestock, it is important to understand how this grazing impacts the saltmarsh ecosystem, and the consequences for ES supply. This thesis (i) uses a global meta-analysis to investigate the effects of livestock grazing on saltmarsh properties, and finds multiple significant changes to soil, vegetation and fauna properties. The meta-analysis reveals that the response of soil carbon is context dependent - there is no effect in Europe but a reduction in the Americas. (ii) Extensive surveys of soil carbon in grazed and ungrazed US marshes, controlling for key covariates, confirm that grazing trades-off against carbon storage in US marshes. These observational surveys, together with 18-month experimental exclusion of horses from a salt marsh in Georgia, show that grazing also disrupts the plant community in US marshes, but has little effect on resident invertebrates. (iii) Focussing on bees in salt marshes, a three-year study in south Wales, UK shows that grazing trades-off against bee habitat by reducing the flower cover of two key food plants, and that increases in plant diversity with grazing do not compensate for this negative effect. (iv) Spatial analyses of seven saltmarsh ES supplied by an estuary complex in south Wales show that marshes are not achieving their potential as a bee habitat here, due to the predominance of grazing. These analyses also show that the provision of ES by salt marshes is spatially heterogeneous, dependent on management, size and location. As a whole, this thesis adds to the understanding of grazer impacts and ES trade-offs, and supplies crucial data to support evidence-based management of salt marshes. 


\section{Declarations and statements}

\section{DECLARATION 1}

This work has not previously been accepted in substance for any degree and is not being concurrently submitted in candidature for any degree.

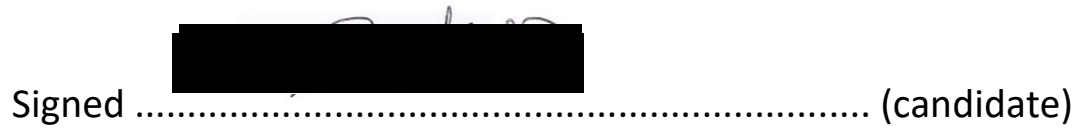

Date .........13/09/2019.

\section{STATEMENT 1}

This thesis is the result of my own investigations, except where otherwise stated. Other sources are acknowledged by footnotes giving explicit references. A bibliography is appended.

Signed (candidate)

Date $.13 / 09 / 2019$

\section{STATEMENT 2}

I hereby give consent for my thesis, if accepted, to be available for photocopying and for inter-library loan, and for the title and summary to be made available to outside organisations.

Signed

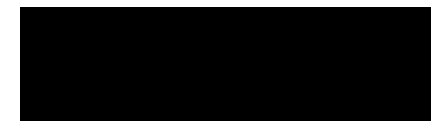

Date $.13 / 09 / 2019$ 


\section{DECLARATION 2}

The following people and institutions contributed to the publication of work undertaken as part of this thesis:

Candidate: Kate Davidson, College of Science, Swansea University

Author 1: John Griffin, Swansea University

Author 2: $\quad$ Mike Fowler, Swansea University

Author 3: Martin Skov, Bangor University

Author 4: $\quad$ Stefan Doerr, Swansea University

Author 5: $\quad$ Nicola Beaumont, Plymouth Marine Laboratory

Author 6: Daniel Forman, Swansea University

Author 7: Jamie Alison, Centre for Ecology and Hydrology

Author 8: $\quad$ Marc Botham, Centre for Ecology and Hydrology

\section{Proportion of work undertaken:}

Chapter 1 (not published)

The thesis introduction was written by the candidate, with feedback from Author 1.

\section{Chapter 2}

This chapter is an abridged version of the manuscript published as Davidson $\mathrm{KE}$, Fowler MS, Skov MW, Doerr S, Beaumont N, Griffin JN (2017). Livestock grazing alters multiple ecosystem properties and services in salt marshes: a meta-analysis. Journal of Applied Ecology.

The candidate and Author 1 conceived the ideas and designed the methodology. The candidate collected and analysed the data, produced data visualisations, and drafted the manuscript, with guidance from Author 1 . Authors 1 and 2 provided guidance on statistical methods. Authors 1, 2, 3, 4 and 5 contributed critically to the drafts.

\section{Chapter 3 (not published)}

The candidate and Author 1 conceived the ideas and designed the methodology. The candidate conducted field sampling, laboratory work and statistical analysis, produced data visualisations, and led the writing of the chapter, with guidance and feedback from Author 1.

\section{Chapter 4 (in review)}

This manuscript is currently under review with Agriculture, Ecosystems and Environment as Davidson KE, Fowler MS, Skov MW, Forman D, Alison J, Botham M, Beaumont N, Griffin JN. Grazing suppresses flowering of key species to reduce bee abundance and diversity on salt marshes.

The candidate conceived the ideas and designed the methodology, conducted salt marsh field sampling, laboratory work and statistical analysis, produced data visualisations, and drafted the manuscript, with guidance from Author 1. Authors 1 , 
2, 3 and 6 provided guidance on methodology for salt marsh surveys. Authors 1 and 2 provided guidance on statistical methods. Author 8 co-ordinated the terrestrial surveys and Author 7 curated the terrestrial survey data for subsequent analysis. Authors 1, 2, 3, 5, 6, 7 and 8 contributed critically to the drafts.

\section{Chapter 5}

Earlier versions of the maps produced in this chapter have been published as Davidson KE \& Griffin JN (2018). Saltmarsh Ecosystem Services in Carmarthen Bay and Estuaries SAC. NRW Evidence Report Series, Report No: 260, 72pp, NRW, Bangor. The candidate and Author 1 conceived the ideas and designed the methodology. The candidate collected the data, conducted statistical analysis, produced data visualisations, and led the writing of this chapter, with guidance and feedback from Author 1.

\section{Chapter 6 (not published)}

The thesis discussion was written by the candidate, with feedback from Author 1.

We the undersigned agree with the above stated "proportion of work undertaken" for each of the above published peer-reviewed manuscripts contributing to this thesis:

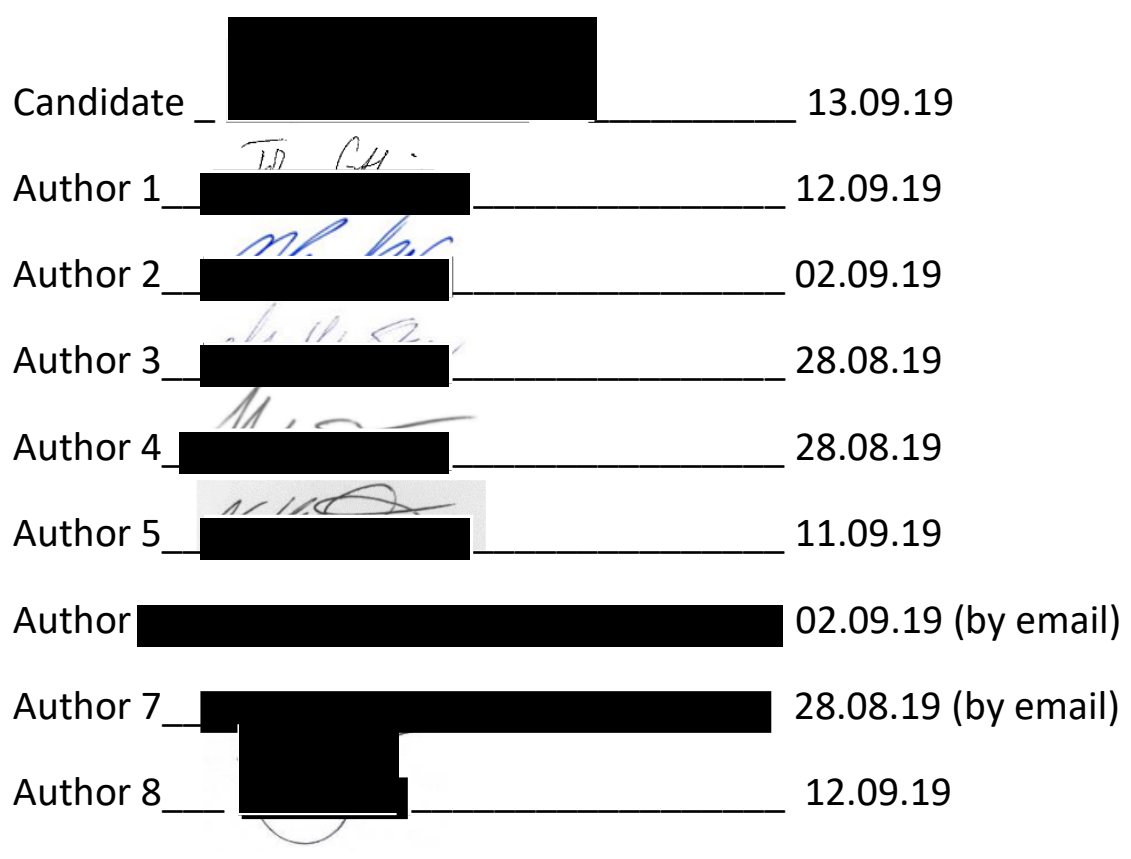


Table of contents

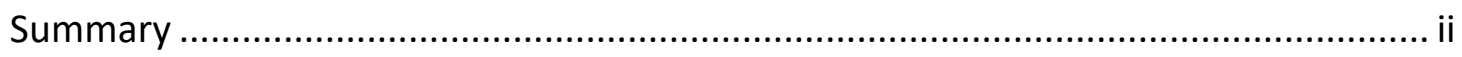

Declarations and statements ...................................................................................

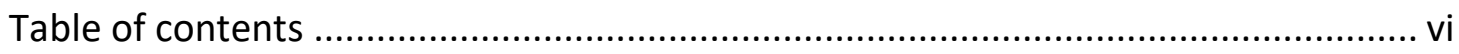

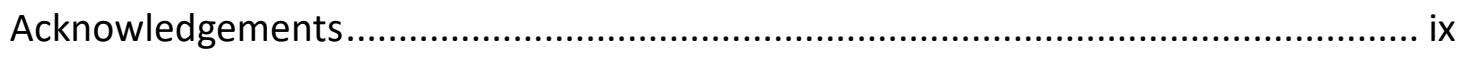

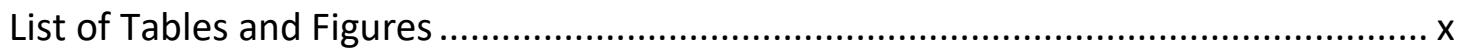

Chapter 1. Introduction: Salt marshes and their ecosystem services ...................... 2

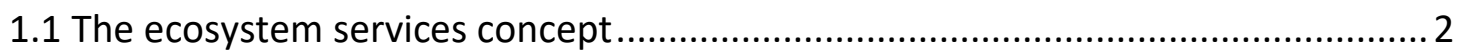

1.2 Multifunctional ecosystems and ecosystem service trade-offs ........................... 4

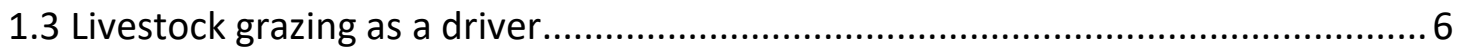

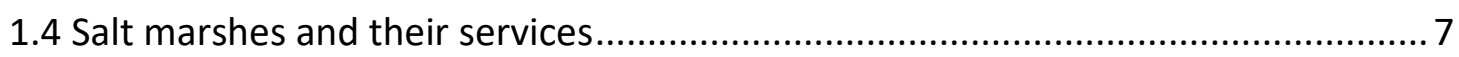

1.5 Livestock grazing in salt marshes ................................................................... 10

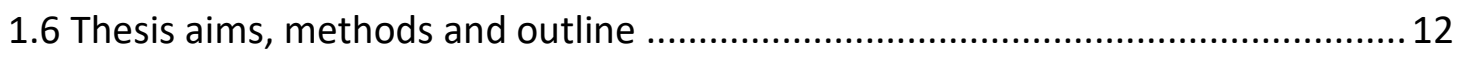

Chapter 2: Livestock grazing alters multiple ecosystem properties and services in salt marshes: a meta-analysis ...................................................................... 14

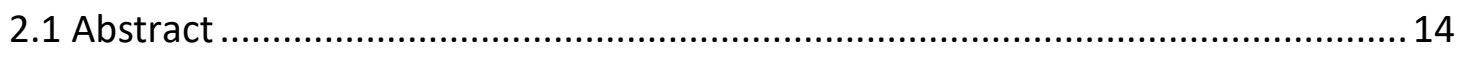

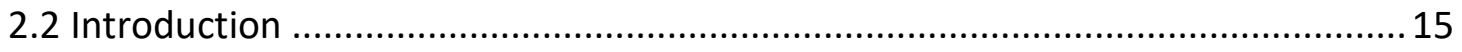

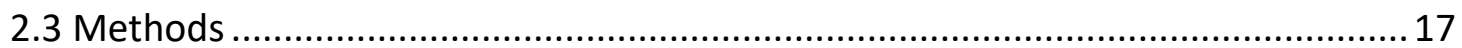

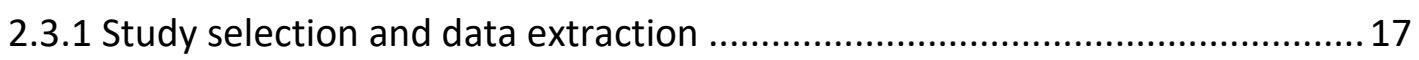

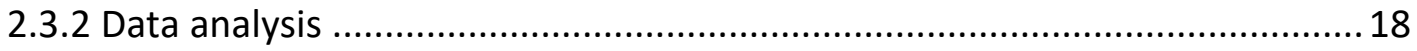

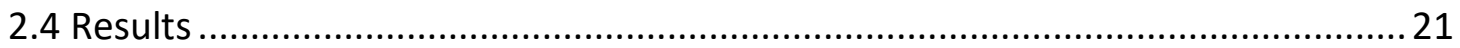

2.4.1. Weighted meta-analysis for mean effects of livestock grazing .................... 22

2.4.2 Coded meta-analysis of all reported outcomes.......................................... 23

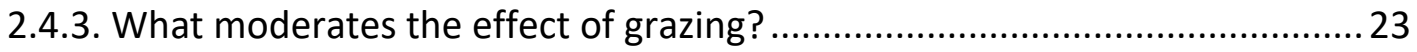

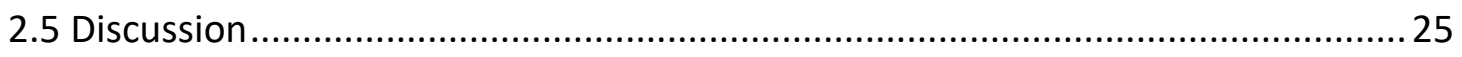

2.5.1 From ecosystem properties to ecosystem services ...................................... 26

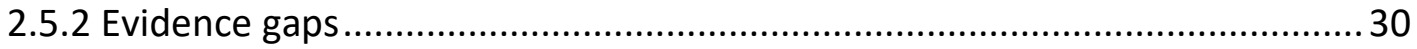

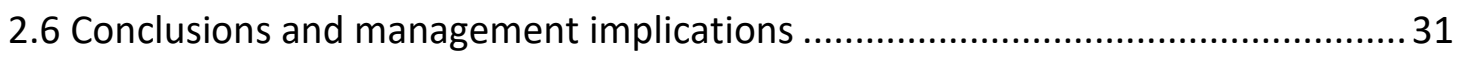

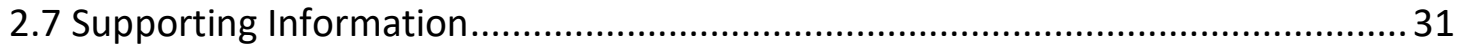

Chapter 3. Large grazers disrupt plant community and reduce soil carbon in US salt

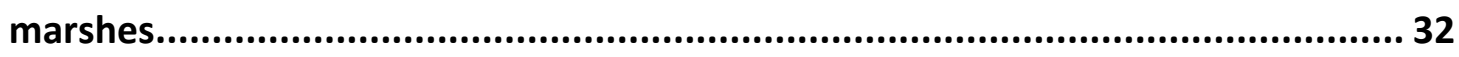

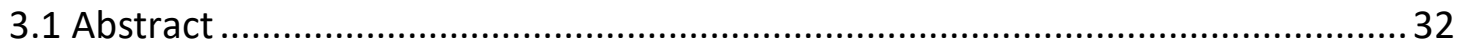




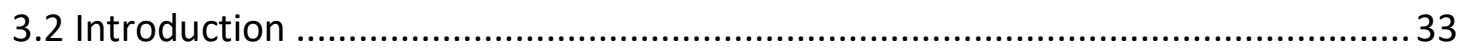

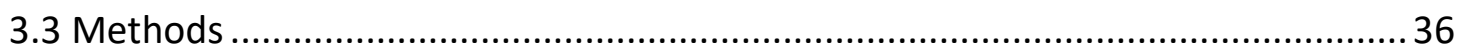

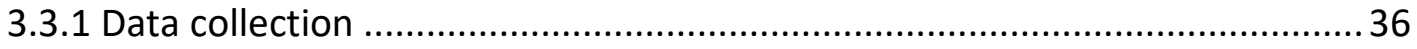

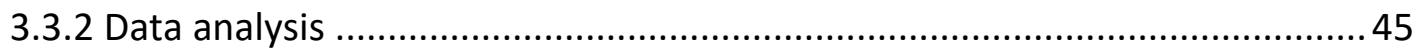

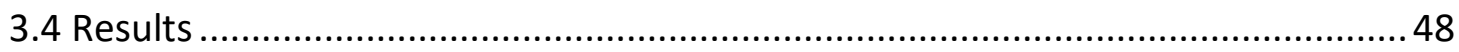

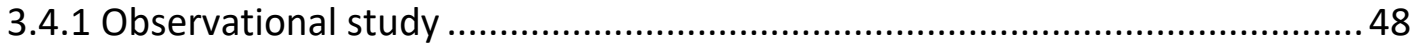

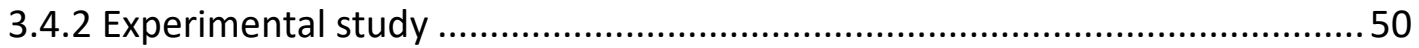

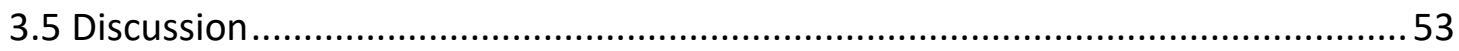

3.6 Conclusions and management implications …................................................. 59

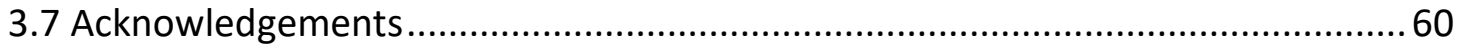

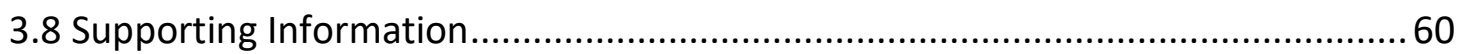

Chapter 4: Grazing suppresses flowering of key species to reduce bee abundance

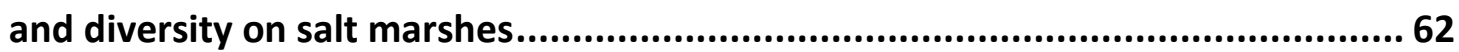

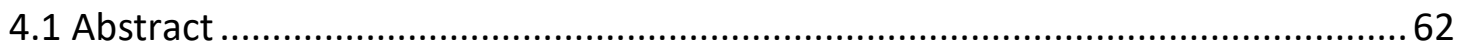

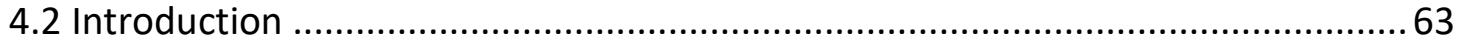

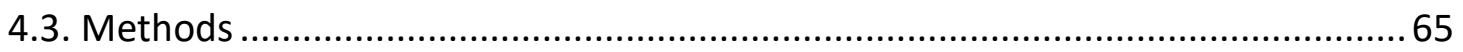

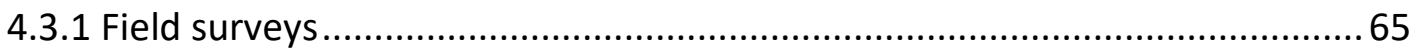

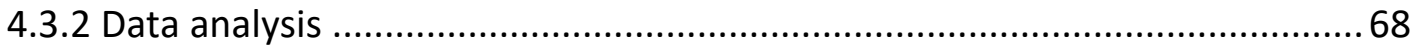

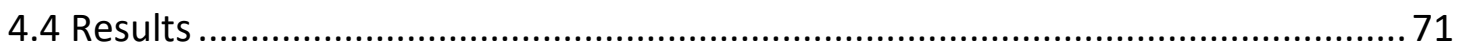

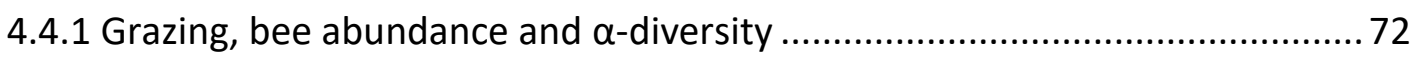

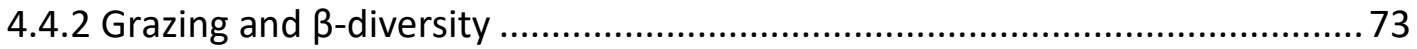

4.4.3 Comparing salt marshes with terrestrial habitats ....................................... 75

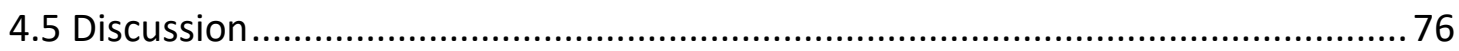

4.6 Conclusions and management implications ….................................................... 79

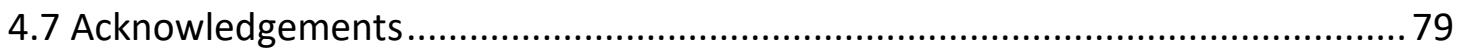

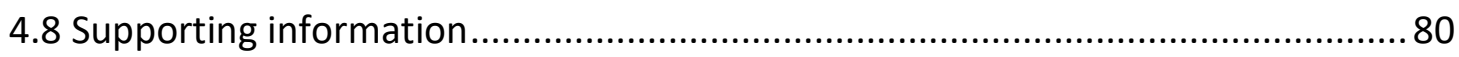

Chapter 5: Mapping ecosystem services, trade-offs and multifunctionality in salt marshes: a case study in south Wales ......................................................... 81

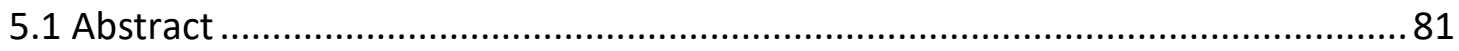

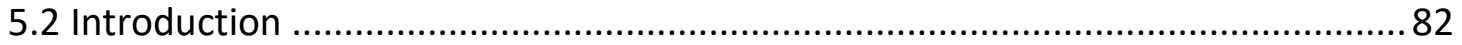

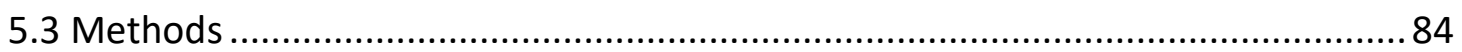

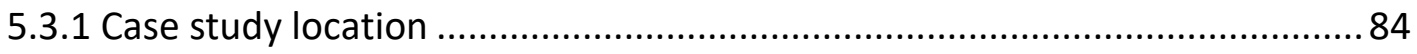

5.3.2 Ecosystem service selection and quantification ......................................... 86 
5.3.3 Multifunctionality and interactions among services .89

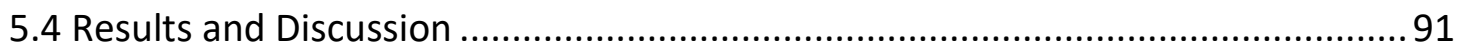

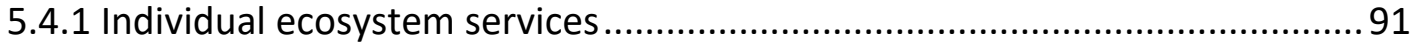

5.4.2 Multifunctionality and interactions among services ................................ 95

5.4.3 Data limitations and recommendations for future study ...........................106

5.5 Conclusions and management implications ..................................................... 107

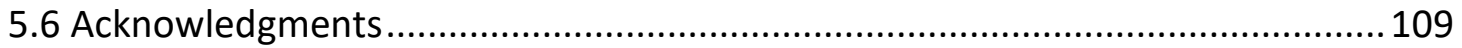

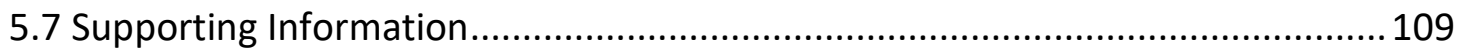

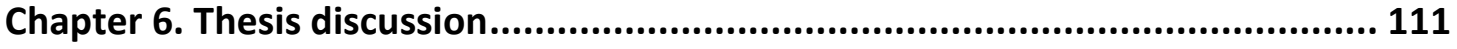

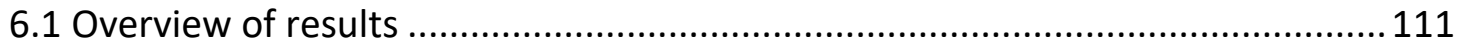

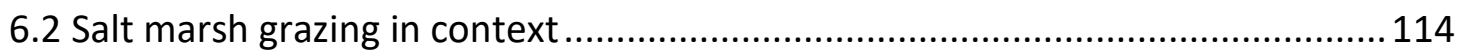

6.3 Reflections on the ecosystem services approach …......................................... 117

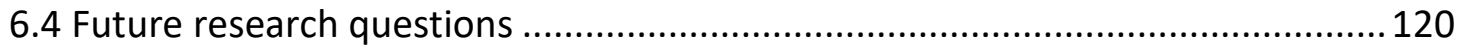

Appendix A: Supporting information for Chapter 2 ........................................ 123

Appendix B: Supporting information for Chapter 3 ......................................... 145

Appendix C: Supporting information for Chapter 4 ........................................... 159

Appendix D: Supporting information for Chapter 5 ......................................... 180

Appendix E: Owner/occupier interviews of saltmarsh management ................... 200

Appendix F: Expert opinion birdwatching survey ............................................. 204

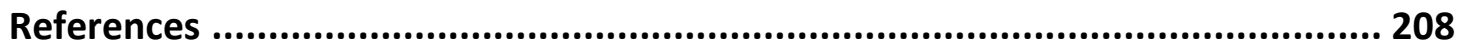




\section{Acknowledgements}

This PhD has afforded many moments of joy, and one of the most joyful aspects has been all the wonderful people that it has been a privilege and pleasure to work alongside. Firstly, thanks to my supervisor, John Griffin, who has offered unlimited support, advice, motivation, inspiration, consolation, understanding, and the freedom to follow my own interests throughout the project. His guidance has been invaluable, and I couldn't have asked for a better supervisor! I would also like to thank my second supervisor, Mike Fowler, who has always been generous with his time and wisdom (especially relating to statistical matters!)

I have received support, advice and assistance from so many people during this $\mathrm{PhD}$ - far too many to mention here. I would particularly like to thank the Resilcoast team for adopting me as an honorary member - I have gained so much from being involved with their group. My studies in America allowed me to work with the Angelini Lab in the University of Florida. The entire lab was so welcoming and helpful, but I would particularly like to thank Christine Angelini and Sean Sharp for their generosity with time, expertise, and spare bedrooms! I have received help from many governmental staff for my projects, but in particular, Doug Hoffman on Cumberland Island has been a real godsend, and helped resolve many difficulties. All my lab-mates in Swansea have been great, for working with, commiserating with, and general good fun. The single regret of my PhD is that, because I stayed living in Cardiff, I didn't get to spend more time with them.

In my personal life, my wonderful friends have kept me sane and distracted, by not giving two hoots about saltmarsh ecosystem services and wanting to talk about anything and everything other than science. My dad has undertaken his own 'Payments for Ecosystem Services' in the form of unasked for, but much appreciated, bank top-ups when my scholarship payments finished. And finally, the biggest thanks to my wonderful husband Kevin James. He has offered emotional, financial and practical support, and has got eagerly stuck into fieldwork and labwork on both sides of the Atlantic. Not once has he expressed resentment at this huge beast that has taken over my (our?) life for the past four years, and I thank him utterly. 


\section{List of Tables and Figures}

\begin{tabular}{lll}
\hline Tables & & Page \\
\hline Table 2.1 & Moderators found to be significant $(P<0.05)$ in regression analyses. & 25 \\
\hline Table 3.1 & $\begin{array}{l}\text { Effect of livestock grazing on key abiotic and biotic variables in salt } \\
\text { marshes }\end{array}$ & 34 \\
\hline Table 3.2 & $\begin{array}{l}\text { Location, grazing history and grazing levels at the 26 surveyed } \\
\text { marshes. }\end{array}$ & 39 \\
\hline Table 3.3 & $\begin{array}{l}\text { Responses measured at Cumberland Island experimental site at 0, 7, } \\
\text { 13 and 18 months. }\end{array}$ & 43 \\
\hline Table 3.4 & $\begin{array}{l}\text { Results of LMMs to predict soil organic carbon (SOC) by grazing } \\
\text { (grazed or ungrazed), soil sand content (\%) and soil clay content (\%). }\end{array}$ & 51 \\
\hline Table 3.5 & $\begin{array}{l}\text { Effect of livestock grazing on properties and processes in salt } \\
\text { marshes. }\end{array}$ & 55 \\
\hline Table 5.1 & $\begin{array}{l}\text { Summary of ecosystem services mapped for the project and upper } \\
\text { and lower boundaries used to rescale values. }\end{array}$ & 90 \\
\hline Table 5.2 & $\begin{array}{l}\text { Results of linear regression analysis testing the effect of marsh } \\
\text { management, area and location on individual ecosystem service (ES) } \\
\text { and multifunctionality measures (MF). }\end{array}$ & 95 \\
\hline Table 5.3 & Marsh characteristics for each ES bundle. & 106
\end{tabular}

\begin{tabular}{|c|c|c|}
\hline Figures & & Page \\
\hline Fig 1.1 & $\begin{array}{l}\text { The ecosystem service cascade from properties to services to } \\
\text { benefits and, ultimately, human well-being. }\end{array}$ & 4 \\
\hline Fig. 1.2 & Salt marsh zonation. & 8 \\
\hline Fig. 1.3 & Global salt marsh distribution. & 8 \\
\hline Fig. 2.1 & $\begin{array}{l}\text { Breakdown of the } 89 \text { studies by a) Continent and country (number } \\
\text { of studies in brackets, some European studies encompassed }>1 \\
\text { country); b) type of grazer; c) study design. }\end{array}$ & 21 \\
\hline Fig. 2.2 & $\begin{array}{l}\text { Weighted mean effects (Log Response Ratio, } \ln R R \text { ) } \pm 95 \% \text { confidence } \\
\text { intervals of livestock grazing on salt marsh properties. }\end{array}$ & 22 \\
\hline Fig. 2.3 & $\begin{array}{l}\text { Regression analyses. Effects of moderators found to be significant in } \\
\text { FDR-corrected analyses. }\end{array}$ & 24 \\
\hline Fig. 2.4 & $\begin{array}{l}\text { Conceptual diagram of how changes in ecosystem properties predict } \\
\text { ecosystem service provision. }\end{array}$ & 26 \\
\hline Fig. 3.1 & $\begin{array}{l}\text { Location of nine observational survey sites along southeast US } \\
\text { coast. }\end{array}$ & 37 \\
\hline Fig. 3.2 & Experimental design on Cumberland Island. & 41 \\
\hline Fig. 3.3 & $\begin{array}{l}\text { The relationship between large mammal grazing and ecosystem } \\
\text { properties at a landscape scale. }\end{array}$ & 50 \\
\hline Fig. 3.4 & $\begin{array}{l}\text { The relationship between grazing intensity and a) stem density and } \\
\text { b) stem height. }\end{array}$ & 50 \\
\hline Fig. 3.5 & $\begin{array}{l}\text { The effect of excluding horses on ecosystem properties after } 18 \\
\text { months. }\end{array}$ & 53 \\
\hline Fig. 3.6 & $\begin{array}{l}\text { Recovery from simulated hog damage in grazed and ungrazed plots } \\
\text { after } 6 \text { months and after } 12 \text { months. }\end{array}$ & 54 \\
\hline Fig. 4.1 & Mechanisms by which grazing may affect bee communities. & 67 \\
\hline Fig. 4.2 & Location of 11 study sites across south Wales, UK. & 68 \\
\hline
\end{tabular}


Fig. 4.3 Model predictions (blue lines) and partial residuals (points) for net grazing effects $(a-c)$ and seasonal effects $(d-f)$ on bees.

Fig. 4.4 The mechanisms of grazing effects investigated via a series of LMMs for $a$ ) all insect-pollinated (IP) plant species combined and $b$ ) key plant species separated out. 75

Fig. 4.5 Plant-pollinator network at each grazing level. 76

Fig. 4.6 Bee abundances in different habitats.

Fig. 5.1 Location of case-study site (Carmarthen Bay) in South Wales, UK (a), and protected designations at study site (b). 86

Fig. 5.2 ES multifunctionality as average ES level. $\quad 98$

Fig. 5.3 ES multifunctionality as Simpson's diversity. 98

Fig. 5.4 ES multifunctionality over a low supply threshold. 99

Fig. 5.5 ES multifunctionality over a moderate supply threshold. 100

Fig. 5.6 ES multifunctionality over a high supply threshold. 101

Fig. 5.7 Correlations between ES and multifunctionality measures. 102

Fig. 5.8 Ecosystem service (ES) bundles within the study area. 104

Fig. 5.9 Distribution of ecosystem service bundles across the study area. 105 


\section{Trade-offs between multiple ecosystem services in UK and US salt marshes}
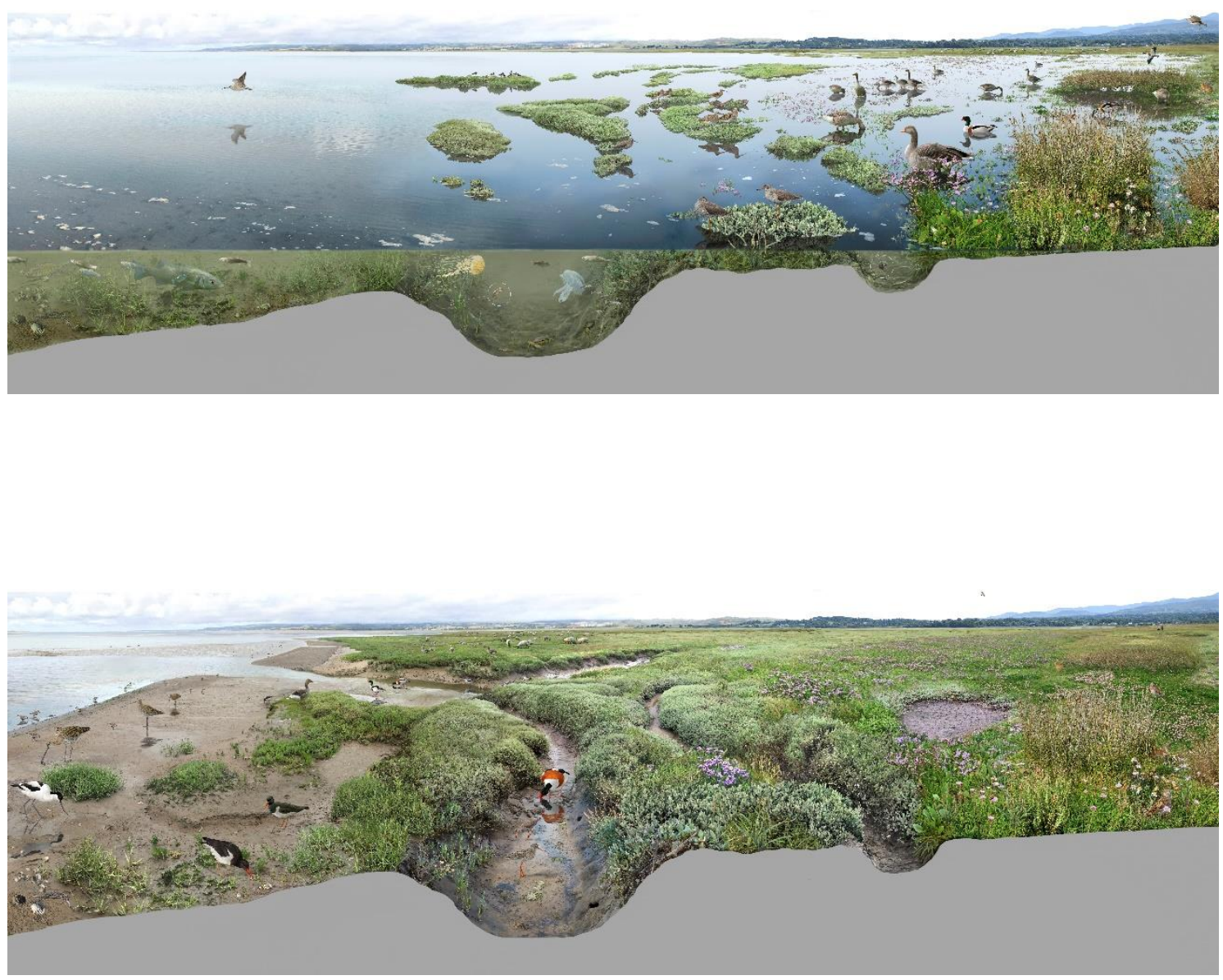

Images of a salt marsh at high and low tide

Illustrations created by @tonixllobet for Resilcoast (http://www.nrn-lcee.ac.uk/resilcoast/) and CoastWEB (https://valuing-nature.net/coastweb). 


\title{
Chapter 1. Introduction: Salt marshes and their ecosystem services
}

\begin{abstract}
Ecosystem services are the conditions and processes through which natural ecosystems, and the species that make them up, sustain and fulfil human life. They maintain biodiversity and the production of ecosystem goods such as seafood, forage, timber, biomass fuels, natural fiber, and many pharmaceuticals, industrial products, and their precursors... In addition to the production of goods, ecosystem services are the actual life-support functions, such as cleansing, recycling, and renewal, and they confer many intangible aesthetic and cultural benefits as well. (Daily 1997)
\end{abstract}

\subsection{The ecosystem services concept}

The concept of ecosystem services (ES) - as material and non-material benefits that people obtain from ecosystems - has a long history, but the term came to prominence in 1997 following the publication of a book titled Nature's Services (Daily 1997) and a Nature article assigning an economic value to the world's ecosystem services (Costanza et al. 1997). The concept gained currency with its adoption by the Millennium Ecosystem Assessment in 2005 (MA 2005a). The MA was initiated by the United Nations and used the ES approach to assess the consequences for human well-being of ecosystem change, and to establish a scientific basis for the actions needed to improve the conservation and sustainable use of ecosystems. The ES paradigm sits at the interface of natural and social science, economics, policy, and industry, and allows ecological, sociological and economic concerns to be considered together, when taking decisions around the management of natural resources. 
The ES approach has attracted controversy, with detractors arguing that it encourages an anthropocentric approach to nature that does not value it for its own sake, prioritises economic considerations above all else, and leaves ecosystems and biodiversity vulnerable to market forces (Monbiot 2014; Silvertown 2015; Chan et al. 2016). Much of this criticism stems from the view that ES analyses are primarily concerned with applying an economic valuation to all aspects of nature, and headline figures such as the huge economic value of crop pollination services certainly capture public attention (France-Presse 2015; Knapton 2015). However, monetisation is not inevitable or necessary for the study of ES values (Boerema et al. 2016). A growing body of ES literature explores non-monetary values held by individuals and communities (Chan et al. 2012; Daniel et al. 2012), and much ES work relates to measuring and communicating the importance of nature to people (Potschin et al. 2016). Applying an economic value to biodiversity can be useful, however, as it allows biodiversity to be incorporated into policy and decision-making, where it may otherwise be ignored (Seddon et al. 2016).

Various frameworks exist to value and categorise ES, although there is a general recognition of a cascade from ecosystem properties, via ecosystem functions and services, to provide goods and benefits that support human well-being (Fig. 1.1). The MA recognised four broad ES categories: provisioning services that provide materials for consumption (e.g. food, water, fuel), services that regulate environmental media or processes (e.g. climate regulation, flood regulation), services that that fulfil cultural needs (e.g. aesthetic, spiritual, recreational), and the supporting services that underpin these other three (e.g. nutrient cycling, soil formation). Some frameworks do not include supporting services in their valuations, classifying these as intermediate ES or ecosystem processes, rather than final ES that provide direct benefits; they therefore exclude supporting services in economic valuations, to avoid double-counting (e.g. Mace et al. 2011). Under different frameworks, biodiversity (or habitat supporting biodiversity) is variously considered as a supporting service (Boerema et al. 2016; TEEB 2019), as a final ES falling across the cultural and provisioning categories (Mace et al. 2011), or as residing outside the four ES categories and underpinning the supply of all services (MA 2005a). In this thesis I have classified biodiversity as a supporting service, as it feeds into multiple final ES, 
and also supports long-term sustainability of ES supply (MA 2005a; Cardinale et al. 2012). This categorisation allows biodiversity and wildlife habitat to be considered as ES in their own right, thus negating some of the criticism of the ES concept, and facilitates ES assessments, as biodiversity/habitat quality is easier and more practical to quantify than many of the final ES that result from biodiversity (e.g. genetic resources, pollination, aesthetic appreciation, spiritual wellbeing).

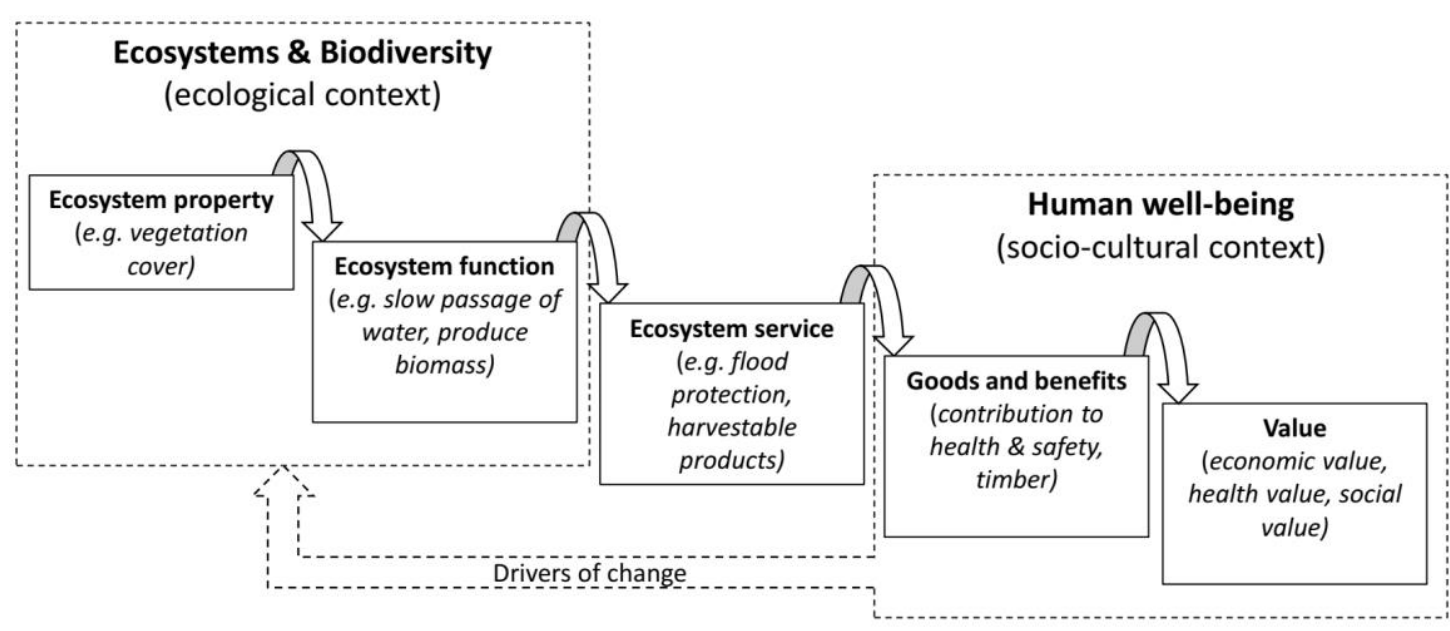

Fig. 1.1. The ecosystem service cascade from properties to services to benefits and, ultimately, human well-being (adapted from de Groot et al. 2010; Haines-Young and Potschin 2010; Mace et al. 2011; Boerema et al. 2016).

\subsection{Multifunctional ecosystems and ecosystem service trade-offs}

Following decades of rapid growth, the world population has reached 7.7 billion, and is expected to exceed 10 billion by 2100 (United Nations 2019). Over the past half century, humans have changed ecosystems more extensively and rapidly than at any time in human history, contributing to substantial gains in human well-being and economic development (MA 2005a). However, $60 \%$ of the ES that humans rely on are degenerating or being used unsustainably, and there has been "substantial and largely irreversible loss in the diversity of life on Earth" (MA 2005a). There is therefore an urgent need to change current policy and practice in the management of natural resources, to secure continuing benefits for future generations and reverse the degradation of ecosystems (MA 2005a). Due to huge pressures from human populations, there is increasing recognition of the need to manage landscapes to 
supply multiple ES concurrently to multiple stakeholders (multifunctionality). Much research is now focussed into exploring the factors that predict ecosystem multifunctionality, particularly biodiversity (Byrnes et al. 2014; Manning et al. 2018). These studies generally explore ecosystem function (EF) multifunctionality, where functioning has no value judgement, rather than ES-multifunctionality, where multifunctionality is valued from an explicitly human perspective (Manning et al. 2018, see Box 1 for definitions). However, because ES flow from EFs (Fig. 1.1) we expect that ES-multifunctionality will relate strongly to EF-multifunctionality, although many of the relationships between EFs and ES are yet to be quantified (Duncan et al. 2015; Bruins et al. 2016; Nilsson et al. 2017).

A key feature of predicting ES-multifunctionality is in understanding how ES interact with each other. ES can interact due to a direct impact of one ES on another, or due to two ES reacting to a common driver (Bennett et al. 2009). Synergies occur where two ES can co-exist at a high level, but trade-offs occur where high levels of one ES preclude high levels of another, or where two ES respond to the same driver in opposite fashions (Bennett et al. 2009). A review of these interactions has shown that trade-offs are more common than synergies, and that trade-offs between food provisioning services and other ES types are particularly common (Howe et al. 2014; Holt et al. 2016). However, the majority of ES publications consider ES in isolation, neglecting complex interaction effects (Boerema et al. 2016). There is a clear need

\section{Box 1.}

Definitions of multifunctionality (from Manning 2018)

We propose that studies should clearly differentiate between (1) measures of multifunctionality including only ecosystem functions, which therefore constitute a metric of the overall performance of an ecosystem, which we term ecosystem function multifunctionality (EF-multifunctionality); and (2) measures that include ecosystem services and where multifunctionality is defined and valued from a human perspective, which we term ecosystem service multifunctionality (ES-multifunctionality). A key distinction between these measures is that EF-multifunctionality attempts to objectively represent overall ecosystem functioning without any value judgement regarding the desired level or types of function, whereas ES-multifunctionality represents the supply of ecosystem services relative to human demand. 
for more research considering the interactions between multiple ES (Boerema et al. 2016). Analysis of spatial concurrence of multiple ES can help to identify trade-offs and synergies, although a lack of overlap in space does not necessarily mean that trade-offs between ES are inevitable, therefore it is important to also investigate the mechanisms underlying these interactions (Bennett et al. 2009). Through understanding the interactions between ES, and the mechanisms underpinning them, managers can seek to enhance synergies and minimise or accommodate tradeoffs, to increase overall ES-multifunctionality across a landscape.

\subsection{Livestock grazing as a driver}

The global demand for livestock products is growing, with accompanying impacts on the environment and ecosystem services (MA 2005b). Livestock production is the single largest user of land, through direct grazing and the production of fodder and feed-grains (MA 2005b). Approximately half of the world's livestock are grazed on dryland rangelands (MA 2005a). Rangelands are natural or semi-natural grazing or browsing lands, comprised of predominantly native vegetation, with low input from humans (EPA 2019). They cover approximately $25 \%$ of the world's land area, and $10-$ $60 \%$ of existing rangeland (depending on biome) is used for livestock grazing (Alkemade et al. 2013). Management of rangelands is primarily concerned with the management of rangeland grazing, and grazing has traditionally been considered the primary driver of rangeland condition (Quirk 2002). The MA listed overgrazing as one of the major concerns related to rangeland degradation, among other threats including land conversion and climate change (MA 2005a). Livestock alter rangeland ecosystems by removing biomass, trampling soil and replacing wild grazers, generally leading to reductions in native biodiversity (Alkemade et al. 2013) and multifunctionality (Ren et al. 2018). However, livestock grazing is not always negative, and in some circumstances, grazing is used to achieve conservation objectives (Lunt et al. 2007). The intermediate-disturbance hypothesis (Grime 1973; Connell 1978) predicts that plant diversity will be low in ungrazed conditions (due to competitive exclusion by dominant species), high under moderate grazing (due to control of dominant species by removal of biomass), and low under heavy grazing 
(due to physiological stress from intense defoliation). This effect has been confirmed in some studies (reviewed by Kershaw and Mallik 2013), and can also cascade to higher trophic levels (Lázaro et al. 2016). However, rangelands cover a wide variety of habitats, including grassland, savanna, wetlands, deserts, tundra, and forb and shrub communities (EPA 2019), each subject to hugely variable biotic and abiotic conditions. Responses to grazing are dependent on many variables, including rangeland type and climate (McSherry and Ritchie 2013; Daskin and Pringle 2016; Hobley et al. 2016; Andriuzzi and Wall 2017). Despite a wealth of research into grazer impacts, there is still no clear picture regarding the relative importance of livestock grazing as a driver in rangelands, and how to define appropriate grazing management within each system. Where rangelands are subject to unique and distinct environmental pressures, such as tidal flooding in salt marshes, there is every chance they will exhibit distinct responses to the pressure of grazing.

\subsection{Salt marshes and their services}

Salt marshes are vegetated wetlands comprised of halophytic grasses, forbs and shrubs, regularly inundated by tides and host to overlapping communities of marine and terrestrial organisms. They are usually intersected by a network of meandering drainage channels and display distinct zonation of plant communities, from those that can tolerate daily inundation of saltwater in the lower regions, to those in the upper zones that are inundated only on the highest spring tides (Fig. 1.2). Salt marshes occur on sheltered coasts across the temperate world, covering approximately 5.5 million ha (McOwen et al. 2017, Fig 1.3), although different regions have distinct flora, fauna and hydrological regimes (Adam 1990; Bakker et al. 2015). For example, in northeastern Atlantic (European) marshes, accretion is primarily by the deposition of sand and sediment during tidal flooding, plant diversity is relatively high, and the dominant top-down control is by domesticated herbivores, which can also facilitate grazing by geese, rabbits and hares (Adam 1990; Bakker et al. 2015). In contrast, northwestern Atlantic (N. American) marshes primarily accrete by the organic accumulation of decaying plant matter, are more expansive, have lower plant diversity, with the major intertidal area dominated by Spartina 
alterniflora, and are subject to strong top-down control by invertebrates (Adam 1990; Bakker et al. 2015).

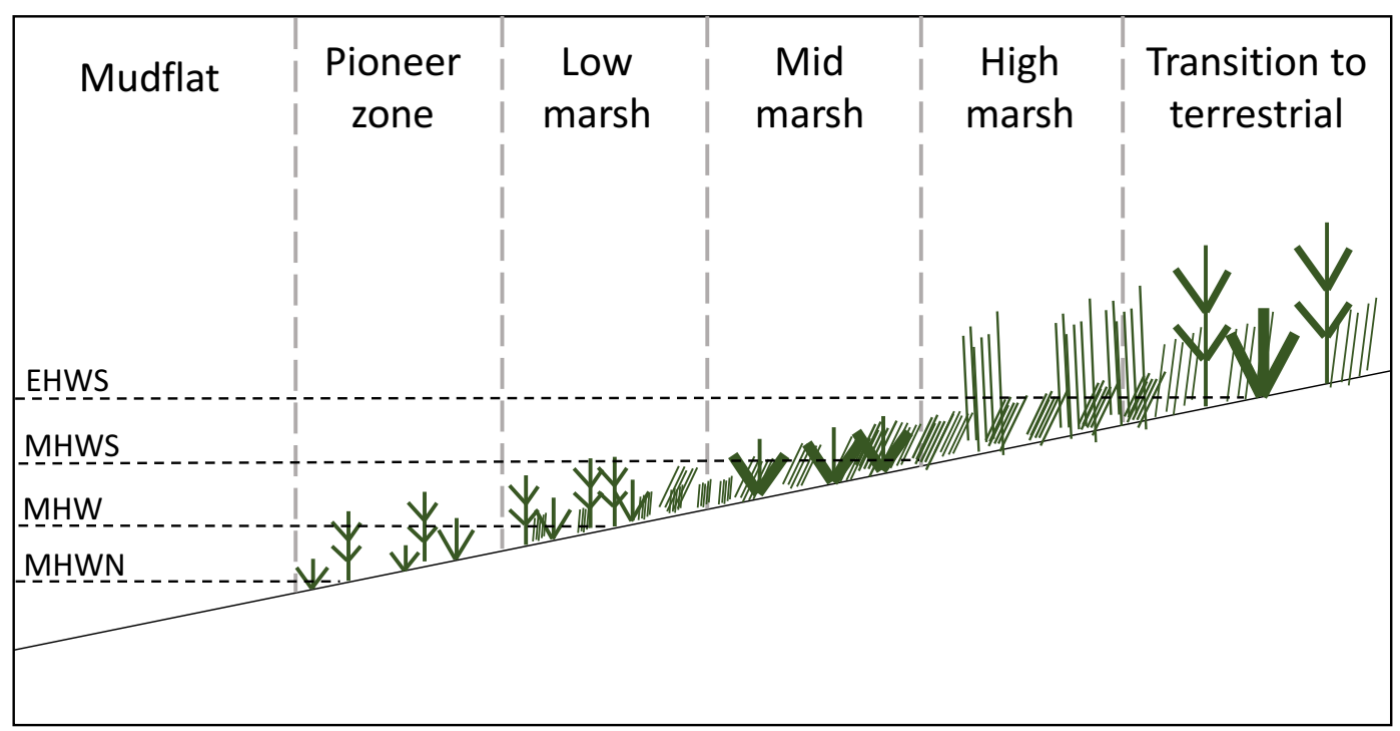

Fig. 1.2. Salt marsh zonation. Representation of saltmarsh zonation driven by tidal inundation. $\mathrm{MHWN}=$ mean high water neap tide, $\mathrm{MHW}=$ mean high water, $\mathrm{MHWS}=$ mean high water spring tide, EHWS=extreme high water spring tide (adapted from Kingham 2013).

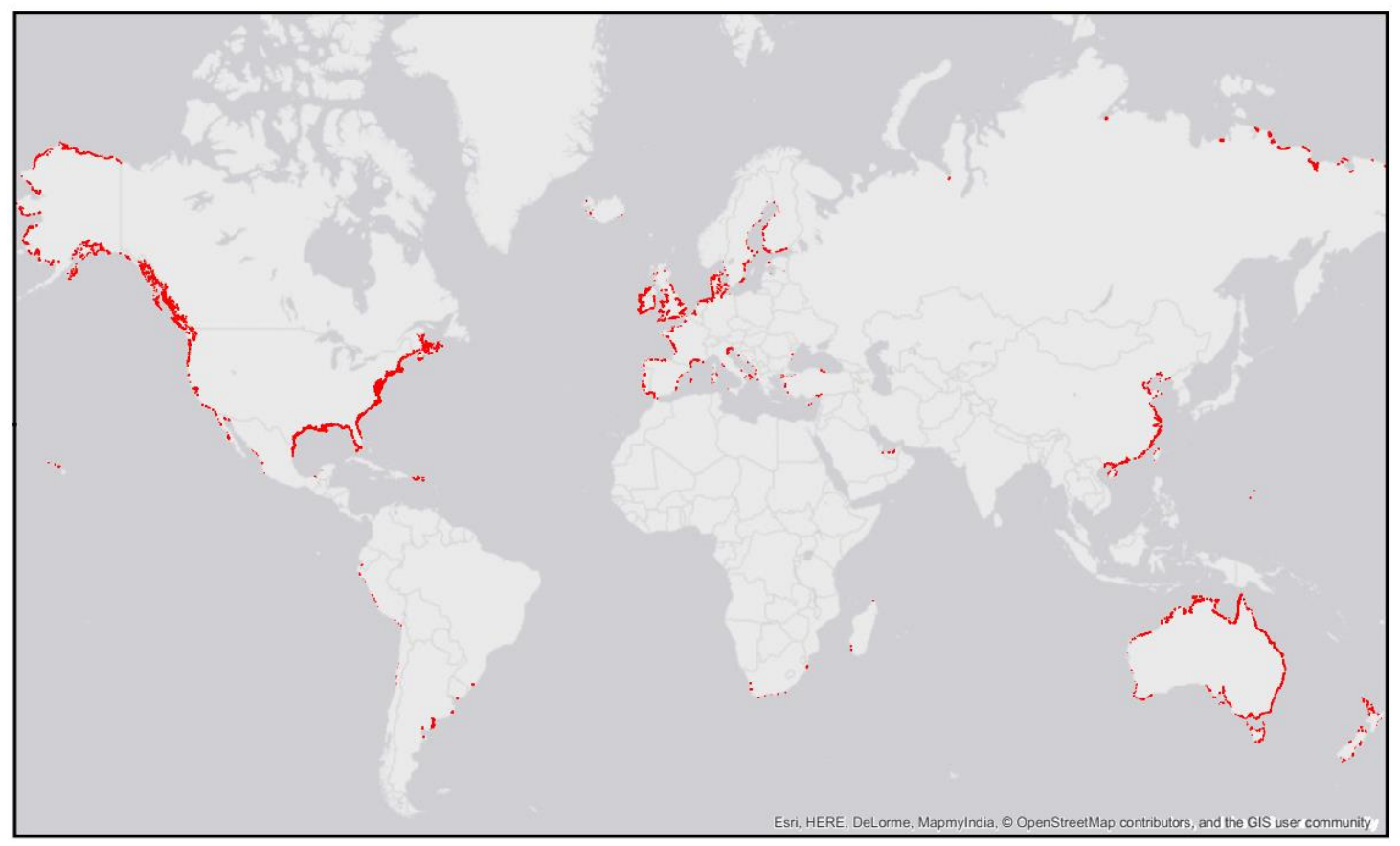

Fig. 1.3. Global salt marsh distribution. Salt marsh extent shown in red (data from McOwen et al. 2017). 
In the arts and popular imagination, salt marsh is commonly portrayed as a dangerous, hostile, even supernatural environment (e.g. VanderMeer 2014; Perry 2016), and historically was often considered a bleak wasteland, home only to mosquitoes and bad odours (Smith et al. 1989; Weis and Butler 2009). A long programme of land 'reclamation' and coastal development has reduced their historical extent by $25 \%$ (McLeod et al. 2011). However, in recent decades, there has been increasing recognition of the varied and valuable services supplied by salt marshes, and their important role within coastal systems (e.g. Costanza et al. 1997; MEA 2005b; Barbier et al. 2011).

Salt marshes yield multiple provisioning services by supplying pastureland for domestic livestock, salt marsh hay, and habitat for wild foods such as Salicornia, wildfowl, fish and crustaceans (Jones et al. 2011). Salt marshes also supply regulating services that help mitigate climate change and other anthropogenic impacts: they sequester and store atmospheric carbon belowground, providing globally important 'blue carbon' sinks (Chmura et al. 2003; McLeod et al. 2011); they offer coastal protection from extreme weather events, estimated to be worth $\$ 8240 \mathrm{ha}^{-1} \mathrm{yr}^{-1}$ in the US (Costanza et al. 2008); and filter nutrients and pollutants from terrestrial runoff (Ribeiro and Mucha 2011; Alldred and Baines 2016). The cultural services of salt marshes are many and varied: they attract bird-watchers, walkers and wildfowlers, offer artistic inspiration, aesthetic beauty and educational opportunities (Jones et al. 2011). Supporting services such as primary production, nutrient cycling, soil formation and biodiversity underly the production of all other services, and the unique characteristics of the saltmarsh environment can enhance these services. For example, salt marshes have high primary productivity as they are unshaded and nutrients are replenished through tidal flooding (Mitsch and Gosselink 2008), underpinning their value as grazing land. The anaerobic conditions in salt marsh soils slow decomposition of organic matter, high sulphates minimise the production of methane, and they continually accrete new sediment and organic matter, maximising their utility for long-term carbon storage (Chmura 2009). Additionally, salt marshes provide a unique habitat for wildlife, supporting abundant and diverse biota (Adam 1990), from which much of their cultural value is derived. 
Salt marshes are subject to multiple anthropogenic pressures, threatening their ES provision and long-term survival. These pressures were reviewed by Gedan et al. (2009) and include resource exploitation and extraction; land reclamation and hydrological alterations such as ditching and the building of roads; industrial and agricultural pollution; overharvesting of predators leading to runaway consumer control; and the introduction of non-native species. Of major concern are the likely impacts of climate change. Rising sea-levels pose an existential threat, potentially leading to the drowning of large areas of marsh (Crosby et al. 2016), while alterations in macroclimate are likely to drive changes in plant community composition, or trigger conversion to mangrove or unvegetated mudflat (Gabler et al. 2017). The increased recognition of the high value of salt marsh, and of the myriad threats risking their functioning and survival, has led to increased protections of saltmarsh habitat, restoration of formerly 'reclaimed' marshes, and an increased drive to sensitively manage the salt marshes that remain for the benefit of both people and nature (Adnitt et al. 2007; Chmura 2009; Roman and Burdick 2012). Livestock grazing is of particular interest for conservation management, as it is one of the easiest ways that managers can effect change in a salt marsh (Lambert 2000; Adnitt et al. 2007).

\subsection{Livestock grazing in salt marshes}

Livestock grazing is the most common resource use of salt marshes (Gedan et al. 2009). European marshes have been grazed by domestic ungulates since pre-historic times (Barr and Bell 2016) and are still widely grazed today (Dijkema 1990), with salt marsh meat obtaining a higher market value than standard products (Jones et al. 2011). However, in some areas, management authorities have excluded livestock for conservation purposes (Bakker et al. 2003). In China, many marshes are intensively grazed (Greenberg et al. 2014), as are those in South America, although here too there is pressure to stop grazing within conservation areas (Costa et al. 2009). In North America, salt marsh grazing is less common (Yu and Chmura 2010), but at several sites there are concerns over the effects of uncontrolled grazing by feral horse populations (Turner 1988; Taggart 2008). 
In salt marshes, poor-quality, non-specific grazing management advice means conservation objectives are often not met (Mason et al. 2019), so there is a clear need for an improved evidence base to inform saltmarsh grazing management. A review into control of coastal vegetation found that studies investigating the effects of wild and domesticated herbivores (top-down effects) are vastly outnumbered by studies investigating bottom-up environmental drivers (He and Silliman 2016). In spite of this unbalanced research focus, there is a long history of studies investigating livestock grazing in salt marshes (Ranwell 1961; Cadwalladr and Morley 1974; Reimold et al. 1975). However, the majority of these studies come from Europe, and particularly the Wadden sea region, where there are several long-term grazing studies (de Vlas et al. 2013). The numerous studies investigating livestock grazing have not previously been quantitatively synthesised, making it difficult to identify consistent trends, or recognise whether the over-weighting of European studies is unduly influencing the general picture that is emerging of livestock impacts. As an example, mechanistic studies of grazing in European marshes indicate that grazing does not trade-off against climate regulation in salt marshes (Ford, Garbutt, L. Jones, et al. 2012; Harvey et al. 2019), but this pattern may not apply in other regions. In terms of conservation grazing, there is considerable research investigating the impact of grazing on vegetation (e.g. Reimold et al. 1975; Jutila 1997; Bouchard et al. 2003; Meirland et al. 2013; Lagendijk et al. 2017), birds (e.g. Laursen 1977; Böhme et al. 1999; Malpas et al. 2013; Cardoni et al. 2015; Sharps et al. 2015) and terrestrial invertebrates (e.g. Armonies 1986; Andresen et al. 1990; Meyer et al. 1995; Böhme et al. 1999; Ford et al. 2013). However, several faunal groups are under-represented, including mammals (Kuijper et al. 2008; Lagendijk et al. 2018), fish (Laffaille et al. 2000; Friese et al. 2018), amphibians (Rannap et al. 2017) and pollinators (Meyer et al. 1995; Rickert et al. 2012; Rickert et al. 2018). The knowledge gap related to saltmarsh pollinators is particularly important from an ES perspective, due to a pressing need to recognise, preserve, and sensitively manage flower-rich habitats in order to prevent a collapse in crop pollination services (Goulson et al. 2015).

Where trade-offs between grazing and other ES are explicitly investigated, these generally concern trade-offs with carbon storage (Ford, Garbutt, L. Jones, et al. 2012; Elschot et al. 2015; Harvey et al. 2019). Investigations into interactions between 
saltmarsh grazing and more than one other ES are extremely rare (but see Ford 2012). This ecological, rather than ES focus, means that the broad societal impacts of saltmarsh grazing are rarely considered. Given the high value and variety of saltmarsh ES, the myriad threats faced by tidal marshes, and the widespread practice of saltmarsh grazing, there is a clear need for a better evidence base on the impacts of livestock grazing on other services, to inform better management of these unique habitats.

\subsection{Thesis aims, methods and outline}

The overall aim of this thesis is to investigate how anthropogenic changes to ecological communities affect multiple ES, using salt marshes as a focal system. Specifically, I will examine the impacts of livestock grazing on saltmarsh properties, functioning, and the supply of other saltmarsh ES. To explore these impacts, I use multiple approaches, including a meta-analysis of the academic literature (Chapter 2), experimental study (Chapter 3 ), observational surveys (Chapters $3 \& 4$ ), interviews (Chapter 5) and analysis of unpublished secondary data (Chapters $4 \& 5$ ). I investigate the effects of grazing at a range of scales, from the global (Chapter 2) through regional (Chapters $3 \& 4$ ) to a local scale (Chapters $3 \& 5$ ).

In Chapter $2 \mathrm{I}$ investigate potential trade-offs and synergies between livestock grazing and other ES, using a systematic review of academic literature reporting ecosystem properties in grazed and ungrazed salt marshes. This chapter analyses data for 29 saltmarsh properties extracted from 89 published studies to obtain, for the first time, a global picture of grazer impacts on salt marshes, identify significant moderators of grazer impacts, and highlight evidence gaps.

Because grazing is less common in US marshes, there are few grazing studies from this region, and many evidence gaps relating to top-down control by large grazers. Chapter 3 uses both experimental exclusion on a grazed island in Georgia, USA, and broad-scale observational surveys in 26 marshes conducted along 1100 km of US coastline, to investigate the impacts of large ungulate grazers in US Atlantic coast salt 
marshes. Chapter 3 builds on and strengthens the results presented in Chapter 2, and adds new data relating to grazer effects on saltmarsh properties and resilience.

There is much published research regarding the effect of livestock grazing on European saltmarsh properties and processes. However, no studies have previously explored the impact of grazers on saltmarsh bee communities. In Chapter 4 I use extensive vegetation and bee surveys, conducted along $121.6 \mathrm{~km}$ of transect in 11 Welsh marshes over three summers, to investigate the effects of grazing management on the quality of foraging habitat for bees, and the mechanisms driving these effects. I also compare these results with terrestrial pollinator survey data, to establish the importance of salt marsh as a bee habitat within the wider landscape.

Although many studies have investigated individual saltmarsh ES, very few investigate interactions between ES in salt marshes, and none have previously mapped multiple services across a saltmarsh landscape. In Chapter 5 I collate and analyse data from charities, government, universities and private individuals to map the supply of seven ES from 3154 ha of salt marsh in Carmarthen Bay, UK. I use these maps to explore the ES-multifunctionality, trade-offs and synergies within this system. Finally, in the Discussion, I consolidate the results from each chapter, briefly consider the practical applications, and discuss the wider context of my results. 


\section{Chapter 2: Livestock grazing alters multiple ecosystem properties and services in salt marshes: a meta-analysis}

Kate E Davidson, MS Fowler, MW Skov, SH Doerr, N Beaumont, JN Griffin

\subsection{Abstract}

The far-reaching impacts of livestock grazing in terrestrial grasslands are widely appreciated, but how livestock affect the structure and functions of sensitive coastal ecosystems has hitherto lacked synthesis. Grazing-induced changes in salt marshes have the potential to alter the provision of valuable ecosystem services, such as coastal protection, blue carbon and biodiversity conservation. To investigate how livestock alter soil, vegetation and faunal properties in salt marshes, we conducted a global meta-analysis of ungulate grazer impacts on commonly measured ecosystem properties (498 individual responses from 89 studies). We also tested stocking density, grazing duration, grazer identity, continent and vegetation type as potential modifiers of the grazing effect. The majority of studies were conducted in Europe (75) or the Americas (12), and investigated cattle (43) or sheep (22) grazing.

All measures of aboveground plant material (height, cover, aboveground biomass, litter) were decreased by grazing, potentially impairing coastal protection through diminished wave attenuation. Soil carbon was reduced by grazing in American, but not European marshes, indicating a trade-off with climate regulation that varies geographically. Additionally, grazing increased soil bulk density, salinity and daytime temperature, and reduced redox potential. Biodiversity responses depended on 
focal group, with positive effects of grazing on vegetation species richness, but negative effects on invertebrate richness. Grazing reduced the abundance of herbivorous invertebrates, which may affect fish and crustaceans that feed in the marsh. Overall vertebrate abundance was not affected, but there was provisional evidence for increases over a longer duration of grazing, potentially increasing birdwatching and wildfowling opportunities.

Synthesis and applications. Our results reveal that the use of salt marshes for livestock production affects multiple ecosystem properties, creating trade-offs and synergies with other ecosystem services. Grazing leads to reductions in blue carbon in the Americas but not in Europe. Grazing may compromise coastal protection and the provision of a nursery habitat for fish while creating provisioning and cultural benefits through increased wildfowl abundance. These findings can inform salt marsh grazing management, based on local context and desired ecosystem services.

\subsection{Introduction}

Livestock are grazed in semi-wild rangelands throughout the world. In terrestrial systems, their impacts on biodiversity and ecosystem properties are now wellestablished (e.g. Tanentzap and Coomes 2012; Alkemade et al. 2013; Daskin and Pringle 2016) together with the determinants of these impacts such as grazer density, type and plant composition (O'Rourke and Kramm 2012; McSherry and Ritchie 2013). However, livestock are also widely grazed in salt marshes - halophytic grasslands distributed along the world's wave-sheltered temperate shorelines which may respond differently due to their distinct soil properties (e.g. higher salinity, lower redox potential), environmental stressors (tidal flooding) and plant communities. Although many empirical studies have measured livestock impacts in salt marshes, a comprehensive synthesis of these studies is currently lacking. Salt marshes are widely recognised for the value of their Ecosystem Services (ES) (Costanza et al. 1997; Barbier et al. 2011), but have suffered large losses in extent and are subject to multiple anthropogenic threats (Gedan et al. 2009). As such, it is vital that remaining areas of salt marsh are managed sensitively to maximise their ES value. 
Livestock pasturage is the most common resource use of salt marshes (Gedan et al. 2009). Large grazers alter the biophysical structures and processes of an environment (ecosystem properties, EPs) via trampling, removal of vegetation, and defecation. These alterations will drive changes in ecosystem functioning, with consequences for the provision of ecosystem services (Haines-Young and Potschin 2010). For example, direct removal of plant material, and direct and indirect effects on biogeochemical cycling can lead to reduced storage of carbon in soils, diminishing the service of climate regulation (Tanentzap and Coomes 2012). These cascading effects enable EPs to be used as proxy indicators for ES provision in the absence of direct measurements of services (Van Oudenhoven et al. 2012). A recent synthesis showed livestock grazing affects salt marsh vegetation properties (He and Silliman 2016). However, equivalent syntheses of grazer effects on belowground properties and faunal biodiversity in salt marshes are missing. To understand how salt marshes and their ES are affected by grazing, it is necessary to analyse a broad range of EPs, and explore how management decisions and other contextual variables will moderate these effects.

Research from terrestrial rangelands has demonstrated that the direction and strength of livestock effects on ecosystem properties is moderated by variables relating to grazing management, such as stocking density and grazer species (Rook et al. 2004; Stewart and Pullin 2008; Paz-Kagan et al. 2016). Other local contextual variables such as climate, soil type and vegetation can moderate the impact of herbivory (He and Silliman 2016). European and American marshes differ in their soil formation (mainly derived from mineral deposits vs mainly derived from organic material, respectively) and vegetation (high diversity vs low diversity) characteristics (Cattrijsse and Hampel 2006; Bakker et al. 2015), which may cause grazing responses to vary between these continents. European saltmarsh vegetation consists of taxa from diverse lineages, with attendant diversity of traits, which may drive differential responses to grazing, depending on the dominating species. For example, grasses are generally more tolerant of grazing than forbs, due to the location of their growing regions (Briske and Richards 1995). Similarly, faunal responses may be moderated by trophic level and clade. Herbivorous invertebrates are likely to suffer most strongly from livestock grazing, as they are in direct competition for the plant biomass 
(Tscharntke 1997). Conversely, grazing wildfowl are likely to benefit, as they favour nutritious, young plant shoots (Lambert 2000).

Here, we conduct a global systematic review and meta-analysis of the effects of ungulate grazers on salt marsh EPs. We analyse 498 responses from 89 studies to identify significant changes in a suite of soil, vegetation and faunal properties. We hypothesise that these responses are moderated by stocking density, grazing duration, grazer identity, continent, vegetation type and faunal functional group. We show that grazing alters 11 out of the 21 EPs tested, and that grazing effects are dependent upon the nature of grazing, geography and vegetation. We use the observed responses to predict how salt marsh grazing impacts on ecosystem functioning and service provision.

\subsection{Methods}

\subsubsection{Study selection and data extraction}

We comprehensively searched published literature using standard techniques (detailed in Appendix A: Supplementary methods). For inclusion, studies must have measured an EP on a grazed and ungrazed area of salt marsh. Only ungulate grazers (hereafter 'livestock') were considered. Both observational and experimental studies were included, as were those that explicitly replicated the effects of livestock by clipping or trampling.

From the figures, tables and text of each study we extracted grazed and ungrazed means, sample sizes and measures of variance (standard deviation, SD; standard error, SE; 95\% confidence intervals, $\mathrm{Cl}$ ) for each EP. The results sections were also scanned for descriptions of changes induced by grazing, even if no mean values were provided. Often, multiple EPs were measured per study, thereby generating multiple grazing outcomes (hereafter referred to as 'entries'). In total, 498 entries for 29 properties were extracted from the 89 included studies (Appendix A: Table A1).

Where possible, study-specific variables were extracted for each entry (detailed fully in Appendix A: Supplementary methods). Potential moderating variables relating to grazing management were recorded: stocking density (converted to a common 
metric of livestock units per hectare, LSU/ha), grazer species and grazing duration (time in years since introduction/removal of grazers). The dominant vegetation in grazed and ungrazed plots was classified as Spartina, other graminoids, or forbs. Marsh zone and sediment type were also noted, but were not tested as potential moderators due to a lack of data.

\subsubsection{Data analysis}

The data were analysed using three different approaches. (i) A weighted metaanalysis, by inverse of variance (Hedges and Olkin 1985), was used to calculate an overall average effect of grazing for every EP that had mean and variance values from $\geq 3$ separate publications. (ii) A coded meta-analysis (Evans et al. 2011) was used to visually summarise all extracted grazing responses, including those that reported only a qualitative description, or reported means without sample size and variances. While only semi-quantitative, due to its inclusiveness, this method provides a wider overview of all studies investigating grazer effects. (iii) For all EPs with $\geq 10$ entries, linear regression models were used to investigate potential moderators for their influence on the effect of grazing. To increase sample sizes, these meta-regressions were unweighted, allowing entries without a reported variance to be included. All analyses were performed using R statistical software version 3.1.2 (R Core Team 2014).

(i) Weighted meta-analysis

For each individual entry, the effect size of grazing treatment was quantified as the log Response Ratio $(\ln R R)$ of the mean of the grazed group $\left(\bar{X}_{G}\right)$ against the mean of the ungrazed group $\left(\bar{X}_{U}\right)$

$\ln R R=\ln \frac{\left(\bar{X}_{G}\right)}{\left(\bar{X}_{U}\right)}$ [Eqn. 1]

The variance for each entry was then calculated as 
$\operatorname{Var}=\frac{S D_{G}^{2}}{N_{G} \bar{X}_{G}^{2}}+\frac{S D_{U}^{2}}{N_{U} \bar{X}_{U}^{2}}$

Where $S D_{G}=$ SD of grazed group, $S D_{U}=$ SD of ungrazed group, $N_{G}=$ sample size of grazed group, $N_{U}=$ sample size of ungrazed group and $S D=\sqrt{N} \times S E$ or $=$ $\sqrt{N} \times \frac{C I}{1.96}$.

When the SD could not be derived from the publication, the variance was estimated as

$\operatorname{Var}_{e s t .}=\left[\frac{N_{G}+N_{U}}{N_{G} N_{U}}\right]+\left[\frac{\ln R R^{2}}{2\left(N_{G}+N_{U}\right)}\right]$ (Hedges \& Olkin 1985).

For each EP, a random-effects, multilevel linear model was used to combine individual effect sizes to estimate an overall mean effect with $95 \% \mathrm{Cl}$. Models were fitted with a restricted maximum likelihood (REML) structure using the rma.mv function within the metafor package (Viechtbauer 2010) in R. Study (i.e. publication) nested within Site was included as a random factor to account for non-independence of multiple entries extracted from the same study, and multiple studies conducted at the same site. In addition, we examined funnel plots to assess publication bias (Sterne and Egger 2001).

\section{ii. Coded meta-analysis}

Entries were coded by the direction and significance of the effect of grazing as causing a statistically significant $(P \leq 0.05)$ increase in the EP, an increase, no change, a decrease, or a statistically significant decrease. Entries were coded as no change when the difference between the grazed and ungrazed means was not significant and $<2 \%$. $P$-values were not always reported, therefore some changes may be recorded as not significant while actually being statistically significant. 


\section{iii. Regression analyses}

To assess potential moderators of the grazing effect, linear, mixed-effect metaregressions were conducted to test whether stocking density $\left(\right.$ LSU ha-1 ${ }^{-1}$, grazing duration (years), grazer identity (sheep; cattle, including water buffalo; mixed species; other), or continent (America; Europe) had a significant effect on the $\ln R R$ of that EP. Within European studies only, vegetation type (graminoid-dominant; forbdominant) was also tested. Spartina spp. were excluded from the graminoid category due to physiological differences ( $C_{4}$ vs $C_{3}$ photosynthesis; Osborne et al. 2014) and habitat preference (Spartina are pioneer species found at the seaward edge of European marshes; Bakker et al. 2015). There were insufficient European Spartina replicates ( 3 studies) to treat it as a separate category, so this vegetation type was not analysed. Because grazing can alter the plant community composition (de Vlas et al. 2013), vegetation type was only included when it was consistent across grazed and ungrazed plots, to allow it to be treated as a predictor of grazing effects, rather than a response to grazing.

There were missing values for each moderator, and frequent collinearity of moderators; as such, each potential moderator was tested for significance in separate models and $P$-values were adjusted for multiple comparisons within that EP using the False Discovery Rate (FDR, Benjamini and Hochberg 1995). Unadjusted Pvalues were also examined, to gain insight into moderators that may potentially be important. All models had Study nested within Site as a random effect. For the EPs of invertebrate abundance and vertebrate abundance, functional group (benthos, detritivore, herbivore, predator; goose, passerine, wader, hare, fish respectively) was included as a random term in each model, to control for varying responses by each group. We also tested functional group as a fixed term in separate models. The majority of studies were conducted at stocking density 0-2.0 LSU ha-1, but two studies were conducted at 6 and 12 LSU ha- $^{-1}$ respectively. Similarly, all studies had a duration of 0.1-100 years, except a single study reporting 210 years of grazing. In these cases, models were run with these outliers (>3 SD from the mean) included and excluded, to determine whether this changed the result. Predictions were only conducted using the models that excluded the outliers, so that these unusual observations did not exert undue influence on the outcomes. 
Models were fitted with a REML structure using the Imer function within the Ime4 package (Bates et al. 2015) in R. Visual checks of residual plots were used to confirm model residuals met assumptions of normality and heteroscedasticity (Pardoe 2012). Model predictions were made using the predictInterval command in the merTools package (Knowles and Frederick 2016) with 1000 simulations, for an unspecified Site and Study. This analysis resamples from the normal distribution of the fixed coefficients, incorporating residual variation to simulate new predictions, and returning a mean prediction and 95\% prediction intervals (PI).

\subsection{Results}

The majority of the 89 studies included were conducted in Europe and over 30\% originated from a single country - the Netherlands (Fig. 2.1a). A variety of grazers were investigated: cattle, sheep, horses, deer and water buffalo, with cattle being most common (Fig. 2.1b). Several manipulative study designs were used (installation of exclosures/enclosures, artificial replication by clipping and trampling, before/after comparison, laboratory study), but over half of the studies were observational (Fig. 2.1c). The duration of grazing ranged from short-term 4-week exclosure experiments, to observational studies in marshes grazed for over 200 years.

a)

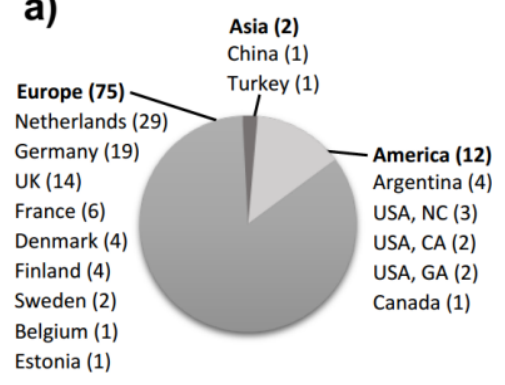

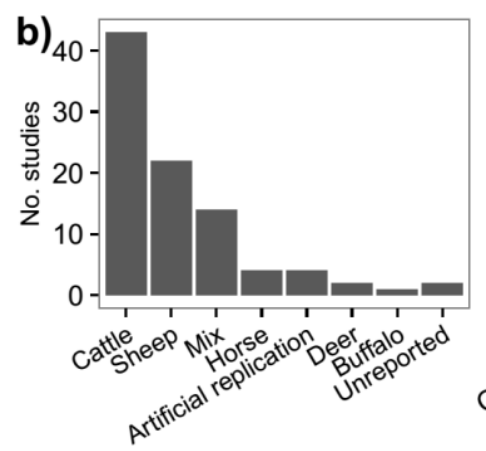

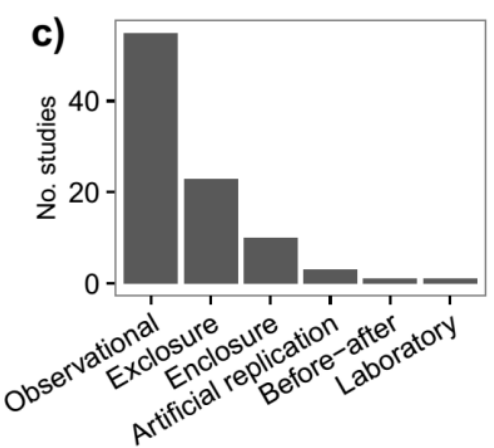

Fig. 2.1 Breakdown of the 89 studies by a) Continent and country (number of studies in brackets, some European studies encompassed $>1$ country); b) type of grazer; c) study design. 


\subsubsection{Weighted meta-analysis for mean effects of livestock grazing}

We found that livestock grazing affected 11 of the 21 EPs tested, spanning soil, vegetation and faunal response variables (Fig. 2.2, Table A2). Grazing significantly altered four of seven soil variables: increasing soil bulk density, salinity and daytime temperature, and decreasing redox potential. Mean accretion rate, soil carbon content and $\mathrm{pH}$ were all unaffected. Grazing also significantly affected five of seven vegetation responses: increasing species richness while reducing aboveground biomass (AGB), cover, canopy height and litter biomass. There was no effect on belowground biomass (BGB) or plant nitrogen content. Grazing was associated with a significant reduction in invertebrate richness, but did not affect vertebrate or total invertebrate abundance. However, when invertebrate abundance data were analysed by functional group, herbivore abundance was significantly reduced by grazing. The majority of the vertebrate data were extracted from studies on bird abundance ( $85 \%$ of entries) and goose abundance in particular (62\%). When goose abundance was analysed separately, the mean effect was positive, but not significant.

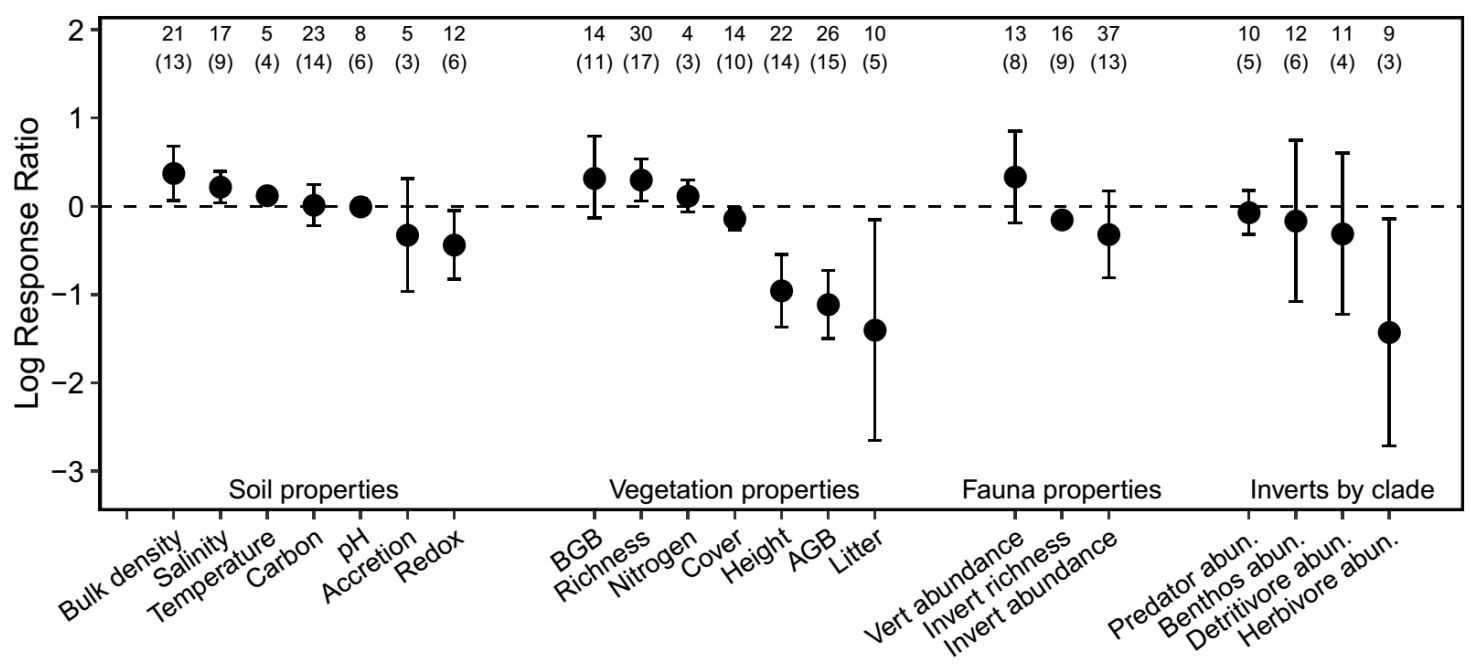

Fig. 2.2 Weighted meta-analysis. Weighted mean effects (Log Response Ratio, $\ln R R$ ) $\pm 95 \%$ confidence intervals of livestock grazing on salt marsh properties. An $\ln R R>0$ indicates a positive effect of grazing on that property, while an $\ln R R<0$ indicates a negative effect of grazing. Effects are significant $(P \leq 0.05)$ where confidence intervals do not intercept 0 . Numbers above points represent number of entries (number of studies). See Table A2 for statistics. 
The ability to detect reporting bias is limited with smaller sample sizes (Sedgwick 2013), but for most properties, no bias was evident from visual assessment of funnel plots (Fig. A1). The exceptions were redox potential, plant cover and plant richness, all of which indicated bias towards reporting of negative effects in smaller, less precise studies (those with a larger standard error). This indicates that the true effects on redox, cover and plant richness may be more positive than our calculated values. Exclusion of 'artificial replication' entries did not alter the direction or significance of the grazing effect for any EP.

\subsubsection{Coded meta-analysis of all reported outcomes}

Results from the coded meta-analysis demonstrate that most EPs have displayed both positive and negative responses to grazing in different studies (Fig. A2). Generally, the balance of responses support the results produced by the weighted meta-analysis. However, the weighted meta-analysis for accretion ( 5 entries) showed no significant effect of grazing, whereas the coded meta-analysis reveals that 11 out of a total 13 entries for accretion showed a negative effect of grazing. Additional patterns were revealed for EPs that could not be analysed statistically in the weighted meta-analysis. Grazing had predominantly negative effects on flowering (8 out of 8 entries) and fish richness/abundance (3 out of 3 ), but had positive effects on stem density (5 out of 6 ) and hare abundance (2 out of 2). Grazing had generally positive effects on wader abundance ( 8 out of 12 ) but negative effects on wader nest survival ( 3 out of 3 ).

\subsubsection{What moderates the effect of grazing?}

Regression analyses adjusted for multiple comparisons

Two moderators that significantly influenced the outcome of grazing were highlighted using linear regression analyses with adjusted $P$-values (Table 2.1). Continent moderated the effect of grazing on soil carbon: grazing is predicted to reduce soil carbon in American marshes but slightly (non-significantly) increase soil 
carbon in European marshes (Fig. 2.3a). Stocking density moderated the effect on canopy height: a higher density of livestock more strongly reduced canopy height (Fig. 2.3b).
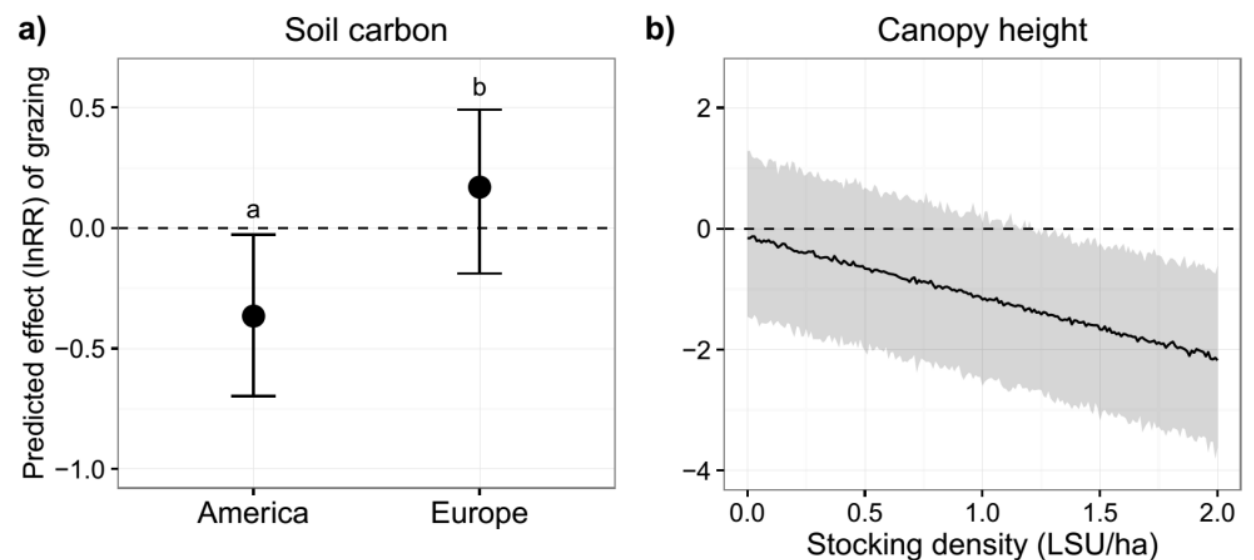

Fig. 2.3. Regression analyses. Effects of moderators found to be significant in FDR-corrected analyses. Predicted effects of a) Continent and b) stocking density on grazing outcomes, with $95 \%$ Prediction Intervals. Different letters indicate categories are significantly different from each other. $\mathrm{LSU} / \mathrm{ha}=$ livestock units per hectare (see Appendix S1 for calculation).

\section{Unadjusted analyses}

Examination of unadjusted $P$-values allowed the identification of other, potentially important moderators (Table 2.1), although these results were considered less robust. The effect of grazing management (stocking density, duration and type of grazer) was significant for five EPs (Fig. A3). Increased stocking density reduced soil salinity and aboveground biomass. Increased grazing duration led to increased vertebrate abundance. Additionally, a positive effect of grazing on BGB was stronger for cattle relative to sheep or a mixture of domestic grazers. For the BGB subset of data, the cattle studies were conducted at a lower stocking density than the sheep or mixture studies, so this result could be an artefact of stocking density (although stocking density was not found to be a significant moderator for BGB when analysed directly). Within European studies, the dominant vegetation type was a significant moderator for two EPs (Fig. A4): areas dominated by forbs experienced larger reductions in percentage cover and species richness than areas dominated by graminoids. 
Table 2.1. Moderators found to be significant $(P<0.05)$ in regression analyses. $n(N)=$ number of entries (number of studies); $\mathrm{df}, \mathrm{F}$ and $P$ show results of ANOVA; FDR- $P$ = False Discovery Rate-adjusted $P$ value; Marginal $R^{2}=$ proportion of variance explained by fixed moderator. FDR- $P$ values $<0.05$ are highlighted in bold. Moderators: stocking density ('LSU'; livestock units per hectare), duration of grazing at site ('Duration'; years), grazer identity ('Grazer'; artificial, cow, sheep, mixed, other), location of study ('Continent'; America, Europe), dominant vegetation type in European studies ('Vegetation'; forbs, graminoids). Functional group ('FG') was also tested for invertebrate abundance (benthic invertebrate, herbivore, predator, detritivore) and vertebrate abundance (goose, wader). The following EPs were tested but had no significant moderators: bulk density*, redox*†‡, litter biomass*, nitrogen content*†‡, invertebrate abundance* and invertebrate richness. Full results of regression analyses, including conditional $\mathrm{R}^{2}$ values, model intercepts, estimates and standard errors are given in Table $\mathrm{A} 3$.

\begin{tabular}{|c|c|c|c|c|c|c|c|}
\hline Ecosystem Property & Moderator & $n(N)$ & df & $\mathbf{F}$ & $P$ & FDR-P & Marginal $\mathbf{R}^{2}$ \\
\hline Soil carbon* & Continent & $27(16)$ & $1,14.8$ & 9.06 & 0.009 & 0.036 & 0.33 \\
\hline Salinity* & LSU & $14(7)$ & $1,11.0$ & 5.84 & 0.034 & 0.136 & 0.33 \\
\hline AGB & LSU & $18(10)$ & $1,15.4$ & 7.76 & 0.014 & 0.070 & 0.32 \\
\hline BGB* $\ddagger$ & Grazer & $14(9)$ & $2,5.9$ & 6.25 & 0.035 & 0.105 & 0.59 \\
\hline Vegetation cover & Vegetation & $10(7)$ & $1,3.3$ & 9.87 & 0.045 & 0.225 & 0.21 \\
\hline \multirow[t]{2}{*}{ Canopy height $\ddagger$} & LSU & $32(16)$ & $1,22.4$ & 12.91 & 0.002 & 0.008 & 0.28 \\
\hline & Duration & $24(12)$ & $1,6.6$ & 6.28 & 0.043 & 0.086 & 0.22 \\
\hline Vegetation richness & Vegetation & 23(14) & $1,21.0$ & 5.05 & 0.036 & 0.180 & 0.19 \\
\hline $\begin{array}{l}\text { Vertebrate } \\
\text { abundance* }\end{array}$ & Duration & $13(7)$ & $1,6.5$ & 5.79 & 0.050 & 0.250 & 0.22 \\
\hline
\end{tabular}

\subsection{Discussion}

We have synthesised four decades of individual studies to highlight key salt marsh properties affected by livestock grazing, including increased plant richness, reduced invertebrate richness and herbivorous invertebrate abundance, reductions in plant material and altered soil conditions. We have also identified previously unappreciated moderating variables that alter the strength or direction of these responses, including an effect of continent on soil carbon and, provisionally, an effect of grazing duration on vertebrate abundance. The findings are applicable to predicting how grazing affects ecosystem functioning and service provision in salt marsh landscapes (see Fig. 2.4 for conceptual diagram). 


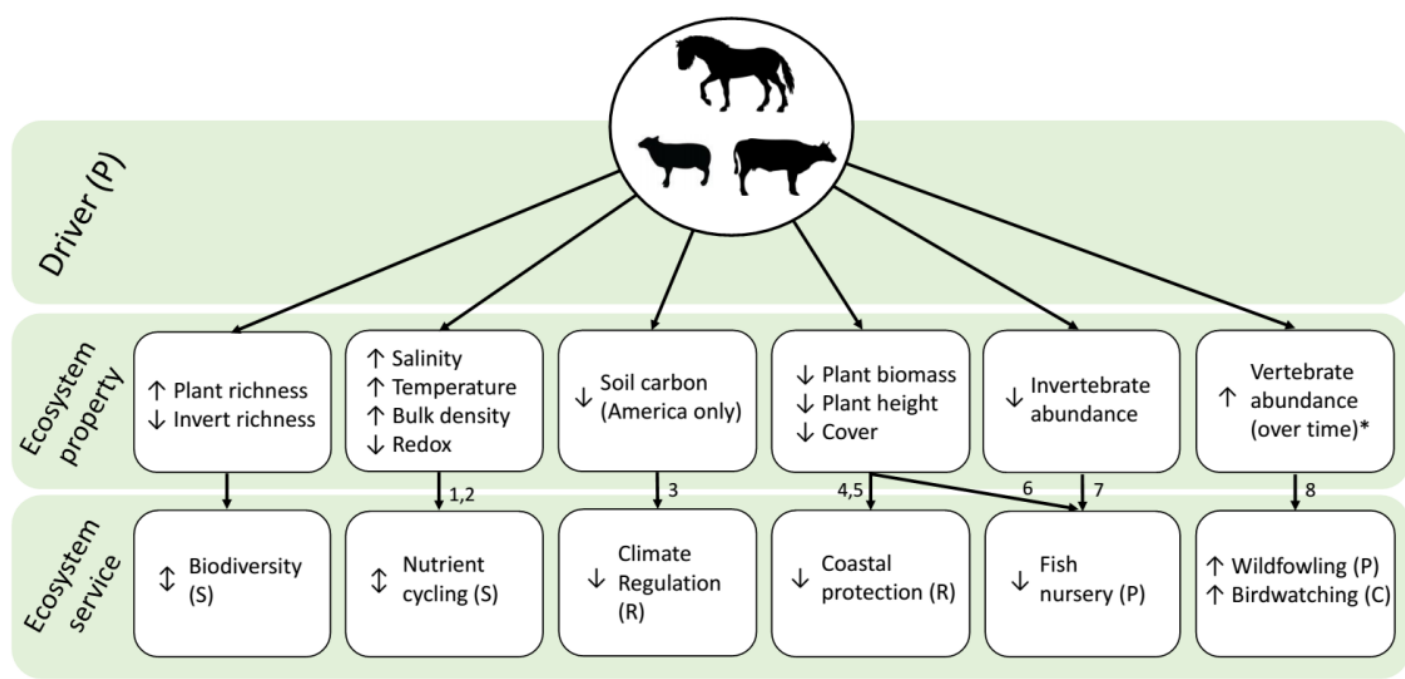

Fig. 2.4 Conceptual diagram of how changes in ecosystem properties predict ecosystem service provision. Services categorised as supporting $(S)$, regulating $(R)$, provisioning $(P)$ and cultural $(C)$. Examples of studies demonstrating ecosystem property - service link are shown as: ${ }^{1}$ Husson 2013; ${ }^{2}$ Wichern et al. 2006; ${ }^{3}$ McLeod et al. 2011; ${ }^{4}$ Möller et al. 2014; ${ }^{5}$ Paul et al. 2016; ${ }^{6}$ Levin et al. 2002; ${ }^{7}$ Cattrijsse and Hampel 2006; ${ }^{8} \mathrm{Green}$ and Elmberg 2014. *This result was not significant after correction for multiple comparisons.

\subsubsection{From ecosystem properties to ecosystem services}

\section{Species richness, soil properties and supporting services}

Biodiversity supports many services and high biodiversity appears to promote ecosystem stability and resilience (Seddon et al. 2016). Extensive grazing is often used as a management method to maintain grassland diversity, as the removal of plant biomass prevents highly competitive species from becoming dominant (WallisDeVries et al. 1998). Our results reveal that grazing is generally beneficial to salt marsh plant richness (Fig. 2.2). However, biodiversity responses were inconsistent: provisional results indicate that increases in richness are only achieved in graminoid-dominated plots (Fig. A4b). Moreover, the overall increase in plant richness was offset by reductions in invertebrate richness and herbivorous invertebrate abundance (Fig. 2.2). These results confirm that responses to land management vary among taxa, and plant richness cannot be used as a broad indicator of biodiversity (Hess et al. 2006). 
Altered soil conditions can drive changes to biotic communities and their functioning, affecting supporting services such as nutrient cycling (Wichern et al. 2006; Husson 2013). Soil bulk density, daytime temperature and salinity all increased with grazing, while redox potential decreased (Fig. 2.2). The increase in bulk density is expected as a direct effect of trampling by large herbivores (Southorn and Cattle 2004; Bell et al. 2011) and this leads to decreased oxygen diffusion and more reduced conditions (Husson 2013). An increase in soil temperature is widely reported from other grazed systems (e.g. van der Wal et al. 2001) as a result of reduced shading, compacted soil and anaerobic respiration. Increased evaporation from warmer, unshaded soils will lead to the observed increase in salinity. Evidence of how these effects will manifest and interact in salt marshes is lacking, and direct measurements of ecosystem functioning are needed to disentangle their mechanisms. Some studies have begun to address grazer impacts on salt marsh biogeochemical cycles (e.g. Olsen et al. 2011; Ford et al. 2012; Schrama et al. 2013), although there were insufficient data to combine in our meta-analysis.

Soil formation in a salt marsh occurs by accumulation of sediment and plant biomass, and allows marshes to accrete vertically in response to rising sea-levels (Bakker et al. 2015; Boyd and Sommerfield 2016). Our analyses revealed that grazers compact the sediment and reduce aboveground biomass, but this did not translate into a significant overall reduction in accretion rates (Fig. 2.2). This may be because grazerdriven compaction increases the strength of the soil, making it more resistant to erosion (Ghebreiyessus et al. 1994). There is also evidence from salt marshes that increased plant richness improves sediment stability (Ford et al. 2016). Therefore grazers may directly and indirectly stabilise the marsh surface and protect against lateral and horizontal erosion. However, accretion rates are highly contextdependent, driven by local factors such as sediment input (Bakker et al. 2015), which may mask the effects of grazing in some studies. In light of the results of our coded meta-analysis (11 out of 13 entries presented negative results for accretion), we recommend further research on the mechanisms and context-dependency of livestock-impacts, as reduced capacity for vertical accretion could lead to submergence under rising seas with concomitant loss in the provision of all services. 


\section{Soil carbon and climate regulation}

In salt marshes, the majority of the carbon stock is stored as soil organic carbon (Murray et al. 2011), so reductions in aboveground biomass are of limited relevance when assessing this service. Overall, soil carbon content was not affected by livestock grazing. However, our analysis revealed that the impact of grazing varied geographically; grazing was found to reduce soil carbon in American marshes, with no consistent effect in the European studies which dominated the dataset (Fig. 2.3). A range of factors could be driving this geographical effect. Reductions in plant material are likely to have a stronger impact on soil quality in organogenic American marshes compared to minerogenic European marshes, where sediment supply will have a stronger effect (Bakker et al. 2015). Moreover, soils in American marshes may be more easily degraded by livestock due to more frequent flooding and a lower stem density compared to European marshes (Cattrijsse and Hampel 2006). American marshes tend to be dominated by Spartina spp., a favoured food plant of livestock (Furbish and Albano 1994), whereas European marshes have a higher floral diversity (Cattrijsse and Hampel 2006), which may confer an increased capacity for grazing resistance (Callaway et al. 2005). The aerial extent of American marshes is an order of magnitude higher than that of European marshes (Ouyang and Lee 2014). Therefore a negative impact of grazing on soil carbon has potential consequences for global storage of 'blue carbon'. Comparative studies in American and European Spartina marshes are needed to determine the variables and mechanisms driving grazer impacts on soil carbon.

\section{Vegetation and coastal protection}

Vegetated coastal regions reduce wave energy more effectively than bare mudflats (Möller et al. 1999; Shepard et al. 2011), with tall, denser vegetation being most effective (Möller et al. 2014; Paul et al. 2016). Unsurprisingly, aboveground biomass, canopy height and cover were reduced in the presence of livestock, with a general trend of stronger effects at higher stocking density or duration of grazing (Fig. 2.3b, Fig. A3) and within forb-dominated plots (Fig. A4a). These alterations could lead to reduced wave attenuation in a grazed salt marsh. However, geomorphological 
characteristics, such as lateral expanse and slope, contribute significantly to wave height reduction (Shepard et al. 2011; van Loon-Steensma and Vellinga 2013). Therefore, the impact of grazing must be considered alongside these known determinants of wave attenuation. Considering the high value of the coastal protection service offered by salt marshes (Costanza et al. 2008), it is worthwhile addressing this grazer effects on wave attenuation through direct field measurements, laboratory study and modelling.

\section{Species abundance and provisioning services}

Provisional results show that vertebrate abundance (predominantly geese) increased with grazing duration (Fig. A3d), indicating that livestock grazing supports the provision of vertebrate prey for wildfowlers. The benefit of longer-term grazing is probably due to the site-fidelity exhibited by migratory birds (Hestbeck et al. 1991). However, there are indications of a trade-off with fish populations, as the three fish studies included in the coded meta-analysis presented negative outcomes of grazing. Decreased herbivorous invertebrate abundance (Fig. 2.2) reduces food resources for juvenile fish and crustaceans, while decreased cover (Fig. 2.2) reduces the shelter value of salt marshes (Levin et al. 2002; Colclough et al. 2005; Kritzer et al. 2016). These effects are likely to be more important in North America than Europe, where marshes are larger and play a greater role as nursery habitat for commercially important fish and crustaceans (Cattrijsse and Hampel 2006).

\section{Cultural services}

In ES research, cultural services are often undervalued or left out altogether, as they are difficult to quantify and are interlinked with both provisioning and regulating services (Chan et al. 2016). The present evidence on how grazing alters EPs nevertheless informs an assessment of cultural services. The provision of optimal wildfowl habitat will promote the conservation of charismatic species and attract birdwatchers (Green and Elmberg 2014). Not all cultural services are likely to benefit from grazing. The presence of livestock may impede access to the marsh, and could alter aesthetic appreciation through changes to floral diversity and abundance (Clay 
and Daniel 2000; Ryan 2011). Conversely, the livestock themselves can act as a tourist attraction and point of interest (van Zanten et al. 2016). Further interdisciplinary research is necessary to assess how appreciation and use of the salt marsh environment may be enhanced or degraded by the presence of grazers.

\subsubsection{Evidence gaps}

These analyses were dominated by European studies. Only one EP (soil carbon) displayed a significantly different response in American marshes. However, there was limited power to detect effects across continents due to the small number of American studies. Additionally, no Australian studies and only one Chinese study were included in this review, despite these countries harbouring a large proportion of the global extent of salt marshes (Ouyang and Lee 2014). Addressing this evidence gap would lead to a more globally representative understanding of livestock grazing impacts in salt marshes.

Due to collinearity of some moderators, and incomplete reporting of study-specific information, we were unable to test for several potentially important moderators (e.g. marsh zone, soil type), nor could we test for interactions between moderators. We did not analyse the effect of plot scale, although this can influence species richness responses in salt marshes (Wanner et al. 2014). We were also unable to assess certain services, such as pollution control and water quality regulation among the most important services provided by salt marshes (Adnitt et al. 2007) and recommend that future work investigate how grazing affects bioremediation in salt marshes. We have used ecosystem properties to inform an assessment of livestock impacts on ES provision, but the links between properties, functions and services are not fully understood. Future research to gain a more mechanistic understanding would facilitate quantitative predictions of the impacts of livestock grazing on ES provision. 


\subsection{Conclusions and management implications}

We have conducted the first meta-analysis of the above- and below-ground effects of livestock grazing in a salt marsh, identifying key patterns that can be used to inform management and direct future research. Reductions in plant biomass, height and cover will diminish coastal defence through reduced wave attenuation, therefore grazing should be carefully managed in salt marshes fronting coastal structures at risk from storm surges. In general, European marshes can be grazed without compromising their blue carbon value. However, we have presented evidence that grazing may impair carbon storage in American marshes. Species richness responses varied by taxa, therefore managers should not use plant richness as a proxy for overall richness. Grazing management for conservation is particularly important as the biodiversity of a salt marsh underpins many services. Ultimately, considering the high value of salt marsh ecosystem services, and the widespread use of these marshes for grazing purposes, further research into the nature of trade-offs and synergies between these services, especially in regions outside of Europe, is strongly recommended.

\subsection{Supporting Information}

The following supplementary material can be found in Appendix A.

Supplementary methods. Study selection and data extraction.

Table A1. Ecosystem properties investigated and list of data sources.

Table A2. Full results of weighted meta-analysis.

Table A3. Full results of regression analyses.

Fig. A1. Forest plots and funnel plots for all EPs.

Fig. A2. Results of coded meta-analysis.

Fig. A3. Predicted moderating effects of stocking density, duration and identity of grazer (significant at $\mathrm{P}<0.05$ ).

Fig. A4. Predicted moderating effects of dominant vegetation in Europe (significant at $P<0.05)$. 


\section{Chapter 3. Large grazers disrupt plant community and reduce soil carbon in US salt marshes}

\subsection{Abstract}

Salt marshes of the US east coast provide multiple ecosystem services (ES). However, many are grazed by horses and other livestock, which may impact ecosystem properties and ES. The majority of grazer-impact studies have been conducted in Europe, and there is evidence that responses in US marshes may be different. To explore how grazing impacts US marshes, I surveyed 14 long-term grazed (horse, cattle) and 12 long-term ungrazed marshes along the US east coast. Long-term grazing reduced soil organic carbon $(\mathrm{SOC})$ in saltmarsh sediments. Long-term grazing also affected multiple plant properties (reduced plant cover and stem height, increased stem density and variation in plant community), but did not impact invertebrates. To control for the effect of environmental variables, and allow for measurement of a larger suite of responses over time, I excluded horses from 8 paired experimental plots on Cumberland Island, GA for 18 months. Horse exclusion led to increased stem height, plant cover and flowering, and decreased algae concentrations. The plant community composition also altered, showing increased Spartina dominance in ungrazed plots. Ungrazed plots had higher densities of adult fiddler crabs (Uca pugnax) but no changes to other crabs, mussels or snails. Exclusion of horses had no clear effect on ecosystem resilience.

Taken together, these results show that grazing consistently disrupts the saltmarsh plant community and structure, but has little effect on resident fauna. Long-term 
grazing reduces soil carbon, but these effects are not apparent following short-term (18 month) changes in grazing regime. Short-term experimental results indicate that resilience of these marshes is not substantially harmed by grazing, therefore the current levels of grazing may be sustainable. However, due to the significant tradeoffs between grazing and carbon storage, and grazing and ES related to plant biomass, horse and livestock grazing should not be practiced on a broad scale in these, or functionally similar, marshes.

\subsection{Introduction}

Salt marshes are extremely valuable to human society, due to their multiple Ecosystem Services (ES) that mitigate environmental hazards, provide material goods, recreational opportunities and wildlife habitat (Costanza et al. 1997; Barbier et al. 2011). Salt marshes are characterised by low plant diversity, but very high primary and secondary production, which drives much of their ES provisioning (Barbier et al. 2011). Grazing livestock on salt marshes can drive significant changes in soil, vegetation and faunal properties (Table 3.1), leading to trade-offs between livestock grazing and other ecosystem services (Chapter 2: Davidson et al. 2017). However, the majority of studies investigating livestock effects have been conducted in European marshes (Chapter 2). There is much geographical variation in saltmarsh flora, fauna, processes and scale (Adam 1990); and as $44 \%$ of the global salt marsh coverage is in North and South America (McOwen et al. 2017), there is a clear need for additional research into livestock effects in American salt marshes. This research is particularly necessary, since the limited data available from the Americas (four studies, three of them observational) suggests that livestock grazing reduces the carbon stored in American saltmarsh soils, in contrast to the neutral effects of livestock in European marshes (Chapter 2). Salt marshes are disproportionally important natural carbon sinks, accounting for 1-2\% of the United States' total carbon sink (Chmura 2009) for $0.21 \%$ of its conterminous land area (McOwen et al. 2017). Sensitive management of this habitat is vital to preserve and enhance this 'blue carbon' service (Chmura 2009; Murray et al. 2011). 
Table 3.1. Effect of livestock grazing on key abiotic and biotic variables in salt marshes. Data summarised from global meta-analysis (Chapter 2). Changes to properties are shown as significantly positive ( $\uparrow)$, no significant effect (-), or significantly negative $(\downarrow)$.

\begin{tabular}{|c|c|c|}
\hline & Property & Response to grazing \\
\hline Soil & $\begin{array}{l}\text { Bulk density } \\
\text { Salinity } \\
\text { Daytime temperature } \\
\text { Organic carbon } \\
\text { pH } \\
\text { Accretion } \\
\text { Redox }\end{array}$ & ( $\downarrow$ in Americas) \\
\hline Vegetation & $\begin{array}{l}\text { Species richness } \\
\text { Belowground biomass } \\
\text { Leaf nitrogen } \\
\text { Cover } \\
\text { Canopy height } \\
\text { Aboveground biomass } \\
\text { Litter }\end{array}$ & $\begin{array}{l}\uparrow \\
- \\
- \\
\downarrow \\
\downarrow \\
\downarrow \\
\downarrow\end{array}$ \\
\hline Fauna & $\begin{array}{l}\text { Vertebrate abundance } \\
\text { Invertebrate abundance } \\
\text { Invertebrate richness }\end{array}$ & ( $\downarrow$ for herbivores) \\
\hline
\end{tabular}

There is a long history of European settlers grazing horses and other livestock on American salt marshes, although this practice is now much declined in the US (Reimold et al. 1975; Smith et al. 1989; Gedan et al. 2009; Gruenberg 2015). However, along the US east coast there are multiple barrier islands - all of them National Seashores, Wildlife Refuges or Reserves - with free-ranging horses (Gruenberg 2015). These horses preferentially graze on saltmarsh vegetation, particularly Spartina alterniflora (Stevens 1986; Wood et al. 1987; Hay and Wells 1991; Furbish and Albano 1994) and a number of studies have suggested that feral horses are causing damage to the natural environment, and recommended the horse populations be reduced (Turner 1987; Turner 1988; Hay and Wells 1991) or removed altogether (Dolan 2002; Taggart 2008). The east coast barrier islands provide shelter to the mainland from storms (Feagin et al. 2010), support commercially important fish (Layman 2000; National Park Service 2014; Baker et al. 2016), and provide important habitat for birds and sea turtles (Erwin et al. 2003; National Park Service 2014). As protected areas, management bodies have a duty to preserve their natural ecosystems and native species, and assess non-native species for their potential for harm (National Park Service 1991). The free-roaming horses are classified as nonnative, feral animals (although some dispute this definition, see Kirkpatrick and Fazio 2010), having been brought to America with European settlers, and then abandoned 
on the islands (Gruenberg 2015). However, removal of the horse populations would be extremely controversial, as 'wild horses' have a key place in American culture, are widely appreciated, have many lobby groups (Gruenberg 2015), and are protected on public land under the Wild and Free-Roaming Horses and Burros Act 1971. It is therefore crucial that there is a sound evidence base underpinning the management of these east coast horse populations.

Of the US studies investigating grazing by horses, all have recorded effects on saltmarsh vegetation (Reimold et al. 1975; Turner 1987; Wood et al. 1987; Hay and Wells 1991; Reader and Craft 1999; Dolan 2002), but fewer have queried the impacts on fauna (benthic infauna: Reader \& Craft 1999; birds, crabs and fish: Levin et al. 2002; fiddler crabs: Reimold et al. 1975; periwinkle snails: Turner 1987), soil properties (C:N:P, bulk density and organic matter: Reader \& Craft 1999), or ecosystem processes (sedimentation: Hay \& Wells 1991). The majority of these investigations have been observational studies comparing neighbouring grazed and ungrazed islands, making it difficult to disentangle the influence of other environmental variables (for example, horses may prefer to graze on firmer, sandier soil types). Saltmarsh fauna, particularly crabs, snails and mussels, can have strong positive or negative effects on ecosystem functioning and resilience (Bertness 1985; McCraith et al. 2003; Silliman et al. 2005; Daleo et al. 2007; Holdredge et al. 2009; Gittman and Keller 2013; Hensel and Silliman 2013; Angelini et al. 2016; Vu et al. 2017; Angelini et al. 2018), so there is a need for further investigation to establish whether the effects of livestock on saltmarsh vegetation cascade down to affect the invertebrate community, saltmarsh multifunctionality, and saltmarsh resilience (the capacity to resist and recover from disturbance, Oliver et al., 2015).

US salt marshes are subject to various threats and disturbances, such as invasive species (e.g. common reed Phragmites australis, feral hogs Sus scrofa), climate change (sea-level rise, drought, increased storminess), and runaway consumer effects (e.g. snails and crabs released from predation) (Gedan et al. 2009). When multiple stressors combine, this can increase the severity of negative effects on vegetation and retard recovery (Silliman et al. 2005; Sharp and Angelini 2016; 
Angelini et al. 2018). If horse-grazing is having strong negative effects on saltmarsh vegetation, this could lead to reduced resilience in the face of other stressors.

In this study, I used a combination of broad-scale observational surveys and experimental exclusion to investigate how large ungulate grazers affect US east coast salt marshes. To assess the impacts of long-term grazing across a geographic range, I measured key biotic and abiotic properties in 14 grazed and 12 ungrazed salt marshes over a 1100 km stretch of coast between Florida and Maryland, southeast USA. To control for the effect of environmental variables, allow for measurement of a larger suite of responses, and assess recovery from grazing over time, I established eight grazer exclusion plots in S. alterniflora salt marsh on Cumberland Island, GA. Here I present the effects on biotic and abiotic properties, and ecosystem resilience, after 18 months of grazer exclusion. I predict that: (i) the long-term observational responses to grazing will match those responses reported in the global meta-analysis, including a negative effect of grazing on soil carbon (Table 3.1); (ii) the short-term exclusion plots will display some responses to grazing, but 18 months of exclusion may not be long enough for all responses to become evident.

\subsection{Methods}

\subsubsection{Data collection}

\section{Observational study}

To assess the effects of grazing across the southeast Atlantic coast, I sampled in 26 marshes at nine sites (e.g. islands or nature reserves) in 5 states along a $1100 \mathrm{~km}$ stretch of coast between $30.52^{\circ} \mathrm{N}$ (Florida) and $38.30^{\circ} \mathrm{N}$ (Maryland) during March and October 2017 (Fig. 3.1, see Table 3.2 for site details). I identified 14 marshes across five sites that were grazed by large ungulates, then selected 12 ungrazed marshes (across seven sites) with similar geomorphology that could act as ungrazed controls. Control, ungrazed marshes were preferably located at the same site, but if this was not possible, I selected marshes at a nearby site $(<25 \mathrm{~km})$ with similar geomorphology. I assessed the grazing level on each marsh as light, moderate or 


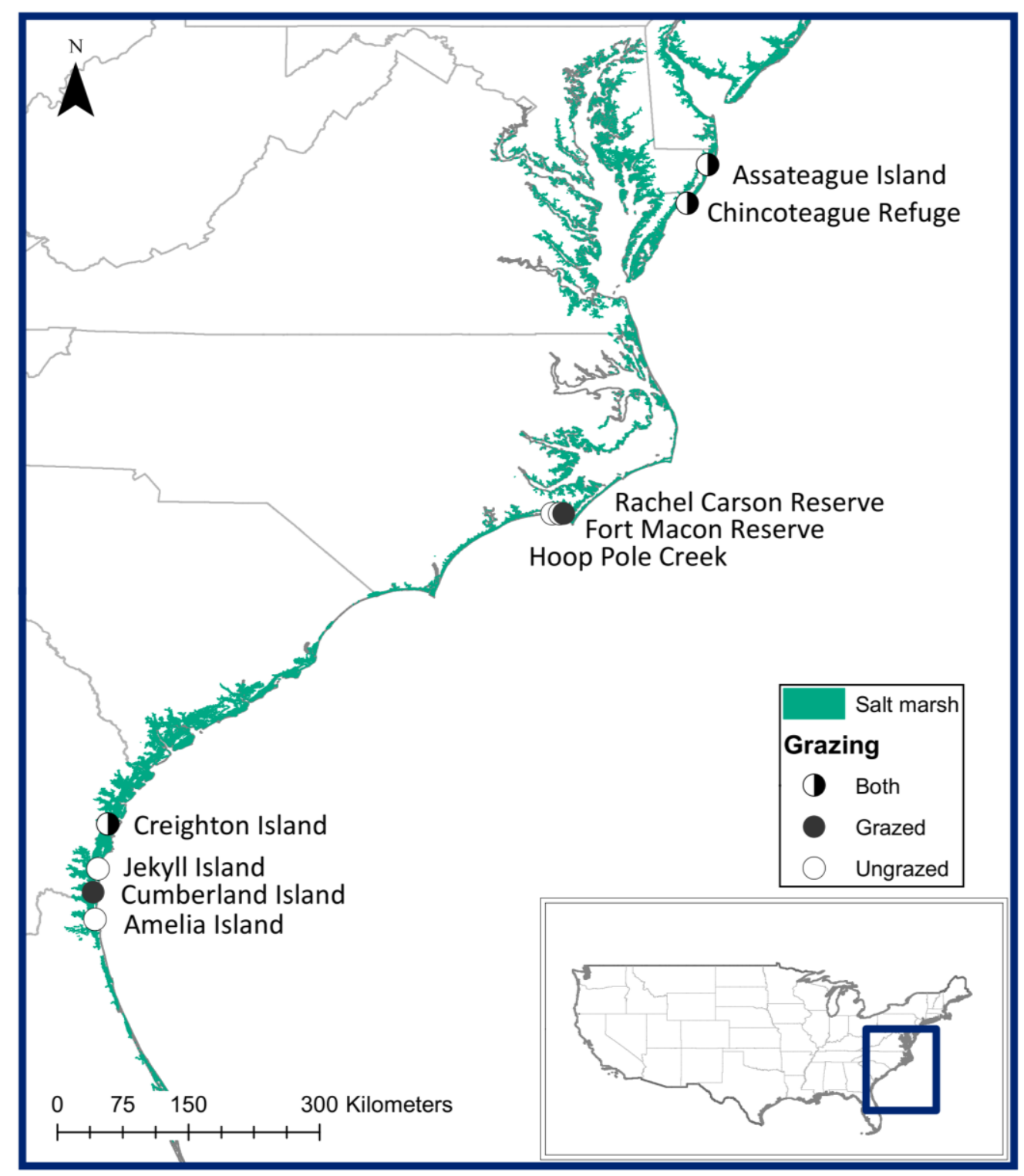

Fig. 3.1. Location of nine observational survey sites along southeast US coast. Each site was surveyed within multiple marshes. See Table 3.2 for details.

heavy based on visual assessment (see Appendix B, Table B1) and information provided by site managers. All of the grazed marshes were grazed by horses except for a single marsh grazed by cattle. I elected to include this cattle-grazed marsh to increase sample size, and because horses and cattle are likely have broadly similar effects due to their similar body size, energy requirements and grazing habits, compared to smaller browsing ungulates and other grazers (Halls 1970; Hubbard and Hansen 1976; Cymbaluk 1990; He and Silliman 2016). Visual inspection of data plots confirmed that cattle-grazed marshes did not produce outliers for any response. 
At each marsh I surveyed along two parallel transects, starting $10-20 \mathrm{~m}$ from the creek edge and running inland. Transects were spaced $20 \mathrm{~m}-200 \mathrm{~m}$ apart, placed to obtain the maximum possible transect length (up to $100 \mathrm{~m}$ ), with separation dictated by natural features such as creeks, areas of maritime forest, and marsh size. Each transect was surveyed within a $1 \times 1 \mathrm{~m}$ quadrat at the start, midpoint and end of the transect. Within each quadrat I measured the redox potential at $2 \mathrm{~cm}$ depth using a portable electrode (Orion Star probe and Accumet Portable OrP Meter, Thermo Fisher Scientifc, Waltham MA, USA), and extracted sediment porewater from $10 \mathrm{~cm}$ depth using a rhizon sampler (Rhizosphere Research Products, Wageningen, The Netherlands) to test for salinity and $\mathrm{pH}$ using a refractometer (Extech Instruments, Waltham MA, USA) and portable pH probe (Mettler Toledo, Columbus OH, USA). I recorded the percentage cover of live vegetation, the proportional cover of all plant species present, the stem length of 15 randomly selected Spartina alterniflora (hereafter Spartina) stems, and the number of live mussels (Geukensia demissa) visible at the surface. Within two $0.3 \times 0.3 \mathrm{~m}$ sub-quadrats, I recorded the stem density, number of marsh periwinkle snails (Littoria irrorata, only those $>5 \mathrm{~mm}$ ), and the number of small $(<5 \mathrm{~mm})$, medium $(5-15 \mathrm{~mm})$ and large $(>15 \mathrm{~mm})$ crab burrows. Burrow densities correspond closely to crab densities in the field, and the three burrow size classes correspond to juvenile Atlantic marsh fiddler crabs (Uca pugnax), adult marsh fiddler crabs and mud/Sesarma crabs (Eurytium limosum, Panopeus obesus, Sesarma reticulatum) respectively (Angelini et al. 2015).

I extracted one soil core ( $7 \mathrm{~cm}$ diameter $\times 15 \mathrm{~cm}$ depth) from each quadrat, divided into an upper $(0-5 \mathrm{~cm})$ and lower portion $(5-15 \mathrm{~cm})$ and stored on ice for subsequent analysis of root biomass, soil organic carbon, and soil texture in the laboratory. The results presented below are for the combined upper and lower portions (i.e. the full 0-15 cm core), as results for the upper and lower strata did not differ from the full core results (see supplementary results, Appendix B). I sampled to a depth of $15 \mathrm{~cm}$, as the upper strata are most likely to be affected by grazers, are most relevant for carbon accumulation (Chmura et al. 2003), and live roots rarely occur at depths below 15-20 cm in Spartina marshes (Blum 1993; Good et al. 1982; Hemminga et al. 1988). 
Table 3.2. Location, grazing history and grazing levels at the 26 surveyed marshes. $N(n)$ shows the number of transects(number of quadrats) surveyed per marsh.

\begin{tabular}{|c|c|c|c|c|c|c|c|}
\hline Site & $\begin{array}{l}\text { Herd size and } \\
\text { access }\end{array}$ & Grazing history & Marsh & Lat, Long & $\begin{array}{l}\text { Grazing } \\
\text { level }\end{array}$ & $\begin{array}{l}\text { Survey } \\
\text { date(s) }\end{array}$ & $N(n)$ \\
\hline \multirow{2}{*}{$\begin{array}{l}\text { Amelia Island, } \\
\mathrm{FL}\end{array}$} & \multirow[t]{2}{*}{-} & \multirow[t]{2}{*}{-} & Al South & $30.52,-81.45$ & - & 06.10 .17 & $2(4)$ \\
\hline & & & Al North & $30.69,-81.45$ & - & 06.10 .17 & $2(4)$ \\
\hline \multirow{3}{*}{$\begin{array}{l}\text { Cumberland } \\
\text { Island } \\
\text { National } \\
\text { Seashore, GA }\end{array}$} & \multirow{3}{*}{$\begin{array}{l}150-160 \text { horses } \\
\text { in } 190 \text { ha } \\
\text { marsh }+6143 \\
\text { ha upland } \\
\text { (most is forest } \\
\text { with no } \\
\text { forage) }^{1,2}\end{array}$} & \multirow{3}{*}{$\begin{array}{l}\text { Grazed by free- } \\
\text { roaming horses \& } \\
\text { livestock for }>200 \text { yrs. } \\
\text { Cattle removed } 20^{\text {th }} \\
\text { century }{ }^{3} \text {. Now only } \\
\text { horses and wild deer, } \\
\text { no herd } \\
\text { management }{ }^{1} .\end{array}$} & Cl Dock & $30.80,-81.47$ & Light & 18.10 .17 & $2(4)$ \\
\hline & & & Cl South & $30.81,-81.47$ & Moderate & 11.03 .17 & $3(23)$ \\
\hline & & & $\mathrm{Cl}$ North & $30.87,-81.45$ & Heavy & $\begin{array}{l}11.03 .17 \\
18.10 .17\end{array}$ & $\begin{array}{l}3(23) \\
2(6)\end{array}$ \\
\hline \multirow{2}{*}{$\begin{array}{l}\text { Jekyll Island, } \\
\text { GA }\end{array}$} & \multirow[t]{2}{*}{-} & \multirow[t]{2}{*}{-} & JI South & $31.04,-81.42$ & - & 29.09 .17 & $2(6)$ \\
\hline & & & JI North & $31.11,-81.42$ & - & 29.09 .17 & $2(6)$ \\
\hline \multirow{2}{*}{$\begin{array}{l}\text { Creighton } \\
\text { Island, GA }\end{array}$} & \multirow{2}{*}{$\begin{array}{l}\text { Unknown size } \\
\text { herd of cattle }\end{array}$} & \multirow[t]{2}{*}{ Unknown } & CR East & $31.50,-81.32$ & - & 28.09 .17 & $2(6)$ \\
\hline & & & CR Grazed & $31.50,-81.32$ & Moderate & 28.09 .17 & $1(3)$ \\
\hline \multirow{3}{*}{$\begin{array}{l}\text { Hoop Pole } \\
\text { Creek, NC }\end{array}$} & \multirow[t]{3}{*}{-} & \multirow[t]{3}{*}{-} & HP East & $34.70,-76.75$ & - & 17.03 .17 & $2(18)$ \\
\hline & & & HP West & $34.70,-76.75$ & - & 17.03 .17 & $2(18)$ \\
\hline & & & & & & 02.10 .17 & $1(3)$ \\
\hline \multirow{2}{*}{$\begin{array}{l}\text { Fort Macon } \\
\text { Reserve, NC }\end{array}$} & \multirow[t]{2}{*}{-} & \multirow[t]{2}{*}{-} & FM East & $34.70,-76.67$ & - & 02.10 .17 & $2(6)$ \\
\hline & & & FM West & $34.70,-76.70$ & - & 02.10 .17 & $2(6)$ \\
\hline \multirow{5}{*}{$\begin{array}{l}\text { Rachel } \\
\text { Carson } \\
\text { Reserve, NC }\end{array}$} & \multirow{5}{*}{$\begin{array}{l}33 \text { horses in } \\
125 \text { ha upland } \\
+109 \text { ha salt } \\
\text { marsh }^{4,5}\end{array}$} & \multirow{5}{*}{$\begin{array}{l}\text { Grazed by feral horses } \\
\text { since } 1940 \text { s when a } \\
\text { small band swam over } \\
\text { from a neighbouring } \\
\text { island. Herd size } \\
\text { formerly up to } 70 . \\
\text { Contracepted to } \\
\text { target } 30^{4} \text {. }\end{array}$} & RC Central & $34.70,-76.63$ & Moderate & 16.03 .17 & $2(18)$ \\
\hline & & & RC Centralb & $34.70,-76.65$ & Moderate & 03.10 .17 & $2(6)$ \\
\hline & & & RC East & $34.70,-76.63$ & Light & 16.03 .17 & $2(15)$ \\
\hline & & & RC Beach & $34.71,-76.65$ & Moderate & 03.10 .17 & $2(6)$ \\
\hline & & & RC West & $34.71,-76.65$ & Heavy & $\begin{array}{l}16.03 .17 \\
03.10 .17\end{array}$ & $\begin{array}{l}2(18) \\
2(6)\end{array}$ \\
\hline \multirow{4}{*}{$\begin{array}{l}\text { Chincoteague } \\
\text { National } \\
\text { Wildlife } \\
\text { Refuge, VA }\end{array}$} & \multirow{4}{*}{$\begin{array}{l}110-140 \text { horses } \\
\text { in two pens } \\
\text { totalling } 1500 \\
\text { ha salt marsh } 6\end{array}$} & \multirow{4}{*}{$\begin{array}{l}\text { Grazed by free- } \\
\text { roaming horses \& } \\
\text { livestock for }>300 \text { yrs. } \\
\text { Livestock removed } \\
\text { mid } 20^{\text {th }} \text { century }{ }^{3} \text {. } \\
\text { Horses restricted to } 2 \\
\text { pens, maintained } \\
<150 \text { adults by annual } \\
\text { auction. Wild deer } \\
\text { present }^{6} \text {. }\end{array}$} & $\begin{array}{l}\text { CH South } \\
\text { Pen }\end{array}$ & $37.89,-75.36$ & Light & 22.3 .17 & $2(10)$ \\
\hline & & & CH South & $37.93,-75.33$ & - & 22.3 .17 & $2(10)$ \\
\hline & & & $\mathrm{CH}$ Central & $37.94,-75.31$ & Heavy & 22.3 .17 & $2(10)$ \\
\hline & & & $\mathrm{CH}$ North & $37.99,-75.30$ & Moderate & 22.3 .17 & $2(16)$ \\
\hline \multirow{4}{*}{$\begin{array}{l}\text { Assateague } \\
\text { Island } \\
\text { National } \\
\text { Seashore, MD }\end{array}$} & \multirow{4}{*}{$\begin{array}{l}65 \text { horses in } \\
2267 \text { ha upland } \\
+1296 \text { ha of } \\
\text { salt marsh }^{5,7}\end{array}$} & \multirow{4}{*}{$\begin{array}{l}\text { Grazed by free- } \\
\text { roaming horses } \& \\
\text { livestock for }>300 \text { yrs. } \\
\text { Livestock removed in } \\
\text { mid } 20^{\text {th }} \text { century }{ }^{3} \text {. } \\
\text { Contracepted to } \\
\text { target } 80-100 . \text { Wild } \\
\text { deer present }^{7}\end{array}$} & AS South & $38.22,-75.15$ & Light & 21.3.17 & $2(18)$ \\
\hline & & & AS West & $38.22,-75.19$ & - & 21.3 .17 & $2(18)$ \\
\hline & & & $\begin{array}{l}\text { AS North } \\
\text { Exclosure }\end{array}$ & $38.30,-75.11$ & - & 21.3 .17 & $2(10)$ \\
\hline & & & AS North & $38.30,-75.11$ & Light & 21.3 .17 & $2(10)$ \\
\hline
\end{tabular}

${ }^{1}$ Doug Hoffman (July 2019), Wildlife Biologist, Cumberland Island National Seashore, pers. comm.;

${ }^{2}$ Dolan (2002); ${ }^{3}$ Gruenberg (2015); ${ }^{4}$ Brandon Puckett (March 2017), Research Co-ordinator, North

Carolina Department of Environment and Natural Resources, pers. comm.; ${ }^{5}$ Taggart (2008); ${ }^{6}$ Kevin

Holcomb (March 2017), Wildlife Biologist, Chincoteague National Wildlife Refuge, pers. comm.; ${ }^{7}$ Bill

Hulslander (March 2017), Chief Resources Management, Assateague Island National Seashore, pers.

comm. 
In the first round of surveys (March 2017) I also recorded vegetation and invertebrate responses (but not sediment responses) in quadrats placed every $10 \mathrm{~m}$ along the transect. On some occasions, due to rising tides, I was unable to sample from every quadrat as planned: in total I collected data from 308 quadrats (113 ungrazed, 57 lightly, 81 moderately, 57 heavily grazed) and 150 soil cores (65, 22, 42, 21). The total number of quadrats and transects surveyed per marsh is shown in Table 3.2 .

\section{Experimental study}

Study site

Cumberland Island is a $29 \mathrm{~km}$ long barrier island located off the coast of Georgia, southeast USA at $30.85^{\circ} \mathrm{N}, 81.45^{\circ} \mathrm{W}$. Cumberland is the largest barrier island in Georgia and the majority of the island was designated a National Seashore in 1972 for its diverse ecology, cultural resources, visitor experience, wilderness, and physical isolation (National Park Service 2014). The island comprises 14743 ha, of which 6 822 ha are marsh, mud flat and tidal creeks (National Park Service 2014). The majority of the National Seashore is accessible to feral horses (Dolan 2002) which have free-ranged on the island since at least the $18^{\text {th }}$ century (Goodloe et al. 2000). The feral horses are unmanaged, have no natural predators, and currently number $150-160$ (D. Hoffman, personal communication, July 18, 2019). Population size is limited by natural death due to starvation, old age, storms and injury. The horses predominantly graze on sand dune and saltmarsh habitat (Dolan 2002), and there is concern that grazing may be causing damage to the natural, protected habitats (Turner 1987; Dolan 2002). The island is also grazed by native white-tailed deer, and is home to feral hogs, although populations of both are controlled by hunting. I studied the effect of horse grazing on multiple ecosystem properties by excluding horses from eight $5 \times 5 \mathrm{~m}$ experimental plots (the maximum size permitted by regulating authorities). The plots were divided between two marshes lying either side of the major creek (Beach Creek) on the southern end of the island (Fig. 3.2a). The plots were erected in March 2017 using 2.5 m metal posts driven into the ground to $1 \mathrm{~m}$ depth, and strung with four strands of high-tensile barbed wire. The plots 
were positioned $15 \mathrm{~m}( \pm 5)$ from the nearest drainage creek, in positions with a similar elevation, vegetation community and faunal community. All plots were positioned in the mid-marsh zone, where short- and medium-form Spartina dominates, because this is the dominant species in east coast US marshes (Adam 1990) and is where the horses predominantly graze. Each $5 \times 5$ m experimental plot was paired with a $5 \times 5 \mathrm{~m}$ control plot, consisting of four corner posts but no barbed wire, positioned $8 \mathrm{~m}$ away. Each pair was spaced $120 \mathrm{~m}( \pm 60)$ from each other. I also established six unmarked reference points, plotted mid-way between each pair. Grazing indicators were assessed at these points and compared with grazing indicators at the control plots, to ensure the corner posts were not acting as an attractant or repellent to the horses.

a)
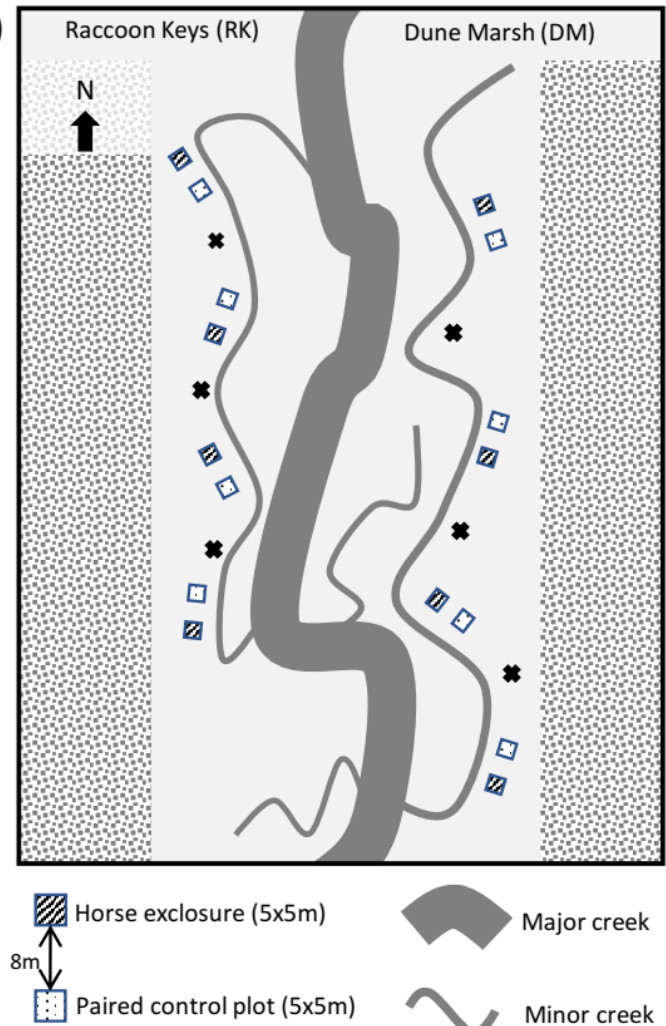

* Unmarked reference point
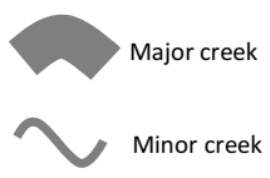

Maritime forest

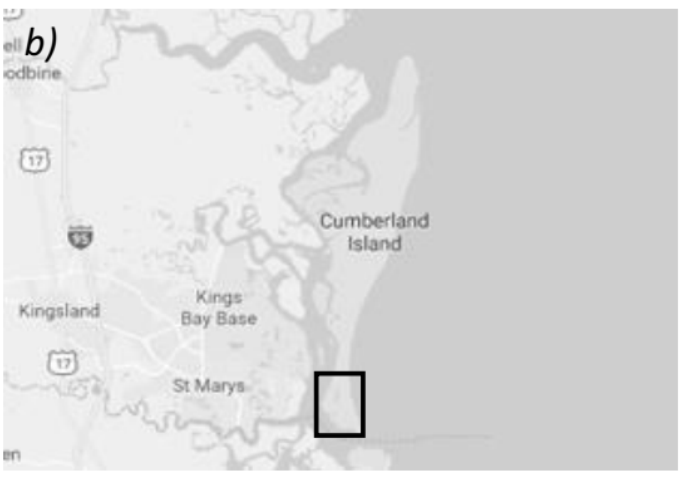

c)

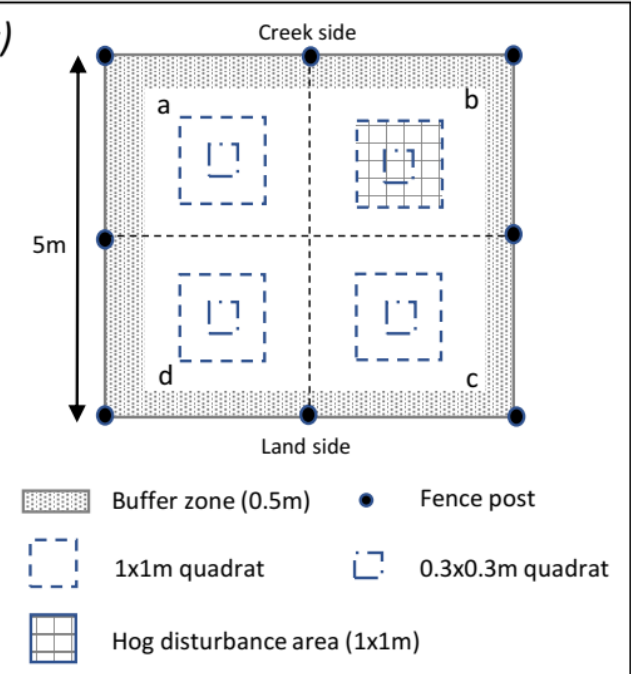

Fig. 3.2. Experimental design on Cumberland Island. Diagrams illustrating a) the distribution of paired plots and reference points in each marsh; b) the location of the study area on Cumberland Island (map data (02019 Google); and c) the sampling regime in each plot in 2018. Not to scale. 
Plots were sampled at 0, 7, 13 and 18 months after establishment (March 2017, October 2017, April 2018, September 2018). At each visit, I collected data on sediment properties, plant properties, the invertebrate community, and ecosystem processes, as summarised in Table 3.3. At 0 and 7 months, I sampled from three quadrats distributed randomly within each plot and used the mean value of every variable for each plot. After one year, each plot was divided into four quarters (a-d), and each plot quarter was sampled independently (Fig. 3.2c). Within each plot, the outer $0.5 \mathrm{~m}$ edge was used as a buffer zone and was never sampled.

Biotic and abiotic responses

I recorded surface elevation using real-time kinematic GPS (Trimble Inc., Sunnyvale, CA, USA) at the corners of each plot at 0 months, and at four points within each plot quarter at 18 months. From every $1 \times 1 \mathrm{~m}$ quadrat, I tested for sediment redox potential, porewater salinity and $\mathrm{pH}$, as described above. I extracted one soil core $(7 \mathrm{~cm}$ diameter $\times 15 \mathrm{~cm}$ depth) from the area just outside each quadrat (so as not to affect the other measurements), divided this into upper $(0-5 \mathrm{~cm})$ and lower $(5-15 \mathrm{~cm})$ strata, and stored in the freezer in ziplock bags for subsequent analysis of root biomass, soil organic carbon, and soil texture in the laboratory.

Within each $1 \times 1 \mathrm{~m}$ quadrat I recorded the percentage cover of living vegetation, the percentage cover of dead vegetation, the proportional live cover of all plant species present, the stem length of 15 randomly selected Spartina stems, and the number of living mussels visible at the surface. I recorded benthic algal concentration (green, blue-green/cyanobacteria, diatoms; three readings of each per quadrat) using chlorophyll-a fluorescence (BenthoTorch, bbe Moldaenke $\mathrm{GmbH}$, Schwentinental, Germany). Within a $0.3 \times 0.3 \mathrm{~m}$ sub-quadrat I recorded the density of living Spartina stems, the number of flowering Spartina stems, and the number of bitten stems (displaying a clean, blunt cut across a leaf). I also counted the number of marsh periwinkle snails, and the number of small $(<5 \mathrm{~mm})$, medium $(5-15 \mathrm{~mm})$ and large (>15 mm) crab burrows. 
Above- and belowground decomposition of primary productivity supports terrestrial and marine foodwebs, nutrient cycling and the structural integrity of saltmarsh soils (Teal 1962; Good et al. 1982). To measure aboveground decomposition rate, I assessed the percentage of mass lost from Spartina 'plugs' left in the field for one month (Hensel and Silliman 2013). To produce these plugs, I harvested standing dead Spartina biomass from both study marshes in April 2018. This biomass was washed and dried (air dried for five days at $\sim 25^{\circ} \mathrm{C}$ then finished in a domestic oven at $\sim 60{ }^{\circ} \mathrm{C}$ for an hour), then three stems were weighed ( $5 \pm 1.3 \mathrm{~g}$ ) and cable-tied to a $60 \mathrm{~cm}$ bamboo pole. I inserted one plug per plot quarter, with the Spartina matter above the sediment level. The Spartina plugs were retrieved after 35 days then washed, dried at $50^{\circ} \mathrm{C}$ for $48 \mathrm{~h}$ and reweighed. To measure belowground decomposition rate, I assessed the percentage of mass lost from teabags buried in the sediment for one month (Keuskamp et al. 2013). In each plot quarter I buried two Lipton green teabags at $5 \mathrm{~cm}$ depth. After 35 days the teabags were retrieved, washed, dried at $50^{\circ} \mathrm{C}$ for $48 \mathrm{~h}$ and reweighed. I also washed, dried and weighed 10 fresh teabags, and used the average value as the pre-decomposition weight.

On each sampling visit I recorded the number of horses present on the marsh at hourly intervals. I assessed grazing intensity by counting hoofprints in each plot, although hoofprint clarity was reduced in muddy or sandy soils, and presence was dependent on tidal cycles. I attempted to assess accretion using an artificial marker horizon (Cahoon and Turner 1989) but the horizon was not visible in soil cores. I also attempted to assess sedimentation using deposition on a filter paper (Nolte et al. 2013a), but many of these filter papers were partially consumed by snails.

\section{Resilience}

After the start of the experiment, some areas of the study marshes were affected by salt marsh die-off (sudden death and decomposition of large areas of Spartina, reviewed by Alber et al. 2008). Therefore, on every visit, I allocated each plot/plot quarter a score of 0-3 for die-off severity (not affected, slightly affected, moderately affected, severely affected). This allowed an assessment of saltmarsh resilience/resistance to die-off under different grazing treatments. To investigate 
saltmarsh resilience/capacity for recovery under different grazing treatments, I simulated feral hog damage within a 1x1 m area in each plot in October 2017. Invasive feral hogs damage standing biomass and soil structure through their trampling, rooting, and wallowing activities (Sharp and Angelini 2016; Sharp and Angelini 2019). I uprooted and discarded 20 clumps of Spartina to simulate root herbivory and vigorously trampled on a square of plastic mesh for $60 \mathrm{~s}$, to simulate wallowing. Data collected from these 'damaged' quadrats (located in quarter ' $b$ ' of each plot) were analysed separately from data collected in undisturbed quadrats.

\section{Laboratory analyses}

Soil cores were oven dried at $65^{\circ} \mathrm{C}$, ground with a pestle and mortar, and sieved through a $2 \mathrm{~mm}$ mesh sieve, separating the root matter from the soil. Both soil and root matter were weighed. I used Loss on Ignition (LOI, ignited at $550^{\circ} \mathrm{C}$ for $3 \mathrm{hrs,}$ Hoogsteen et al. 2015) to determine the organic matter content (\% of dry mass lost) of a $5 \mathrm{~g}( \pm 0.01 \mathrm{~g})$ subsample of the homogenised soil sample. I converted this value to soil organic carbon concentration using the relationship for saltmarsh soils established in North Carolina (Craft et al. 1991):

$$
\text { Organic carbon }=0.40[\text { LOI }]+0.0025[\text { LOI }]^{2}
$$

Soil cores collected from October 2017 onwards were analysed for soil texture using the United States Department of Agriculture (USDA) soil texture analysis protocol (USDA 2017 adapted from Thien 1979). This protocol uses grittiness, stickiness and a 'ribbon test' of a wetted soil sample to allocate soils to one of 11 texture classifications. I then used a soil texture triangle (Fig. B1) to assign each texture class a value for percentage clay and percentage sand content. All soil cores collected in 2018 were additionally tested for soil moisture content (\% of dry mass) by weighing the soil core prior to and after oven drying. 


\subsubsection{Data analysis}

\section{Observational study}

To explore how long-term grazing affects ecosystem properties across a wide range of sites, I used linear mixed models (LMMs) to test how grazing affects multiple sediment (salinity, $\mathrm{pH}$, redox, sand and clay content, soil organic matter), plant (root biomass, living plant cover, plant species richness, stem height, stem density) and invertebrate (periwinkle, juvenile fiddler crab, adult fiddler crab, mud/Sesarma crab, and mussel density) responses in the observational study sites. All LMMs were fitted with grazing (present or absent), season, distance from creek and latitude as fixed effects, and marsh nested within site as a random effect. I tested whether grazing intensity was a significant factor by repeating all models with grazing intensity (ungrazed, light, moderate, heavy) instead of grazer presence as a predictor.

I performed all models on z-transformed data, to allow calculation of standardised effect sizes and coefficients, and comparison of each response on the same scale. Model assumptions were verified by examining residual plots (scaled residuals versus predicted values and versus each covariate in the model) and testing goodness of fit (Kologorov-Smirnov) of observed versus expected values. Response variables were log-transformed where necessary to meet model assumptions. I used likelihood ratio tests (LRTs) using maximum likelihood (ML) estimation to test the significance of each fixed effect and used post hoc Tukey tests for pairwise comparison of intensity levels when grazing intensity was found to be significant.

I was unable to test the effect of grazing on the proportional cover of each plant species, as the highly skewed data violated model assumptions. Instead, I used a binomial generalised LMM to test whether grazing (presence and intensity) significantly affected the probability of a quadrat being $100 \%$ Spartina, while controlling for the fixed effects of season, distance from the creek, and latitude, and the random effect of marsh nested in site. To further explore whether grazing affected the plant community, I used Permutational Multivariate Analysis of Variance (PERMANOVA, Anderson 2001) on pairwise dissimilarity matrices calculated from presence-absence species data (Sørensen dissimilarity) and species percentage cover data (Bray-Curtis dissimilarity), to test whether grazing caused significant variation in 
species composition. Because PERMANOVA is sensitive to differences in within-group dispersion, I also ran a permutation-based test for homogeneity of multivariate dispersions (PERMDISP, Anderson, 2006).

Soil organic carbon (SOC) is positively correlated with fine-textured, high-clay soils (Hu et al. 2014; Bai et al. 2016; Kelleway et al. 2016) and in the observational soil cores there was a significant positive correlation between SOC and clay content (Pearson's $r=0.42, t=4.10, d f=80, P<0.0001$ ). I hypothesised that large grazers may avoid high-clay areas due to the difficulty of walking here, and would instead target more sandy areas for grazing. Therefore, to account for the potentially confounding co-variance of grazer presence and soil texture I tested competing models to predict SOC with (G) grazing, (S) sand content, (C) clay content, (GS) grazing + sand content, (GC) grazing + clay content, as well as a null model (0). I tested the significance of each predictor using LRTs and compared each model's AIC value to determine which factors are most important to predict SOC. All LMMs predicting SOC additionally controlled for the fixed effects of season, distance from the creek, and latitude, and the random effect of marsh nested in site.

\section{Experimental study}

Baseline tests

To test whether control and experimental plots differed at the start of the experiment, I used linear regression models (LMs) for every variable recorded at $\mathrm{T}=0$ (Table 3.3), with treatment (grazed control, ungrazed experimental) and plot pair as predictors. I also tested whether the control plots experienced similar grazing levels to the rest of the marsh using linear (mixed) models with each vegetation and grazing variable (Table 3.3) as the response and treatment (grazed control, unmarked reference point) and marsh as the predictors. Each response was tested the start of the experiment $(T=0)$, and after installation of the plots ( $T=7 \& 18$ months, with plot as a random effect to control for repeated measures at each plot). 
Effect of grazing on ecosystem properties

To test whether horse grazing affects saltmarsh ecosystem properties, I used linear mixed models (LMMs) to test every variable measured at $\mathrm{T}=18$ months (Table 3.3) with treatment (grazed control, ungrazed experimental) as the predictor and plot pair as a random effect. For variables that showed a significant difference between grazed and ungrazed plots at $\mathrm{T}=18$, I explored how quickly these variables responded to the altered grazing regime, using data collected at all time points, and allowing an interaction of treatment and time as the predictor and plot pair as a random effect. I then performed post hoc comparisons of the effect of grazing treatment at each time point, using Tukey's HSD test (Abdi \& Williams 2010).

Effect of grazing on recovery from damage

To investigate how horse grazing affects the capacity of a salt marsh to recover from simulated hog damage, I compared four plant responses in grazed control, grazed damaged, ungrazed control and ungrazed damaged quadrats. I used LMMs to test the interaction between grazing treatment and damage on living plant cover, dead plant cover, stem height, and stem density, while controlling for the random effect of plot pair. I tested each of these plant responses 6 months after and 12 months after the hog damage was simulated. I used post hoc Tukey tests to compare damaged and undamaged quadrats within and across grazing treatments to explore two types of recovery: (i) across-grazing recovery (absolute recovery) - has the damaged vegetation in grazed plots recovered to the same state as the damaged vegetation in the ungrazed plots?; and (ii) within-grazing recovery (relative recovery) - has the damaged vegetation recovered to the same state as undamaged vegetation under the same grazing treatment?

All statistical analyses were conducted in R Studio running R3.5.2 (R Core Team 2018). (G)LMMs were fitted using Ime4 (Bates et al. 2015) and $R^{2}$ values were calculated using piecewiseSEM (Lefcheck 2016). I used the emmeans package (Lenth et al. 2019) to perform post hoc pairwise contrasts of factor levels and calculate 
predicted means for graphing. Multivariate analyses were conducted using the vegan package (Oksanen et al. 2019).

\subsection{Results}

\subsubsection{Observational study}

I analysed ecosystem properties from 14 grazed and 12 ungrazed marshes spread over nine sites. I found that grazing was associated with significant differences in three sediment and three plant properties, but not with any invertebrate properties (Fig. 3.3, Table B2). Grazing was associated with increased stem density, but reduced plant cover and stem height. The effects of grazing on stem density and stem height strengthened with increased grazing intensity (Fig. 3.4, Table B3). Raw plant species composition data (Fig. B2) indicate that grazing is associated with reduced Spartina cover and increased cover of other species (Salicornia, Distichlis, Juncus, Batis), although the increased species richness and reduced probability of a grazed quadrat being $100 \%$ Spartina was not significant (Table B2). Grazing did significantly affect the plant community composition, irrespective of whether a presence/absence or abundance related community dissimilarity measure was used (PERMANOVA F=3.66, $\mathrm{P}=0.039$ and $\mathrm{F}=4.89, \mathrm{P}=0.014$ for Sørensen and Bray-Curtis dissimilarity matrices, respectively). Multivariate tests of group dispersions indicate that these differences are due to increased dispersion within grazed marshes, i.e. the plant communities within grazed marshes are more dissimilar than the plant communities within ungrazed marshes (PERMDISP $F=4.28, \mathrm{P}=0.044$ and $\mathrm{F}=6.79, \mathrm{P}=0.008$ for $\mathrm{S} \varnothing$ rensen and Bray-Curtis dissimilarity matrices, respectively). 


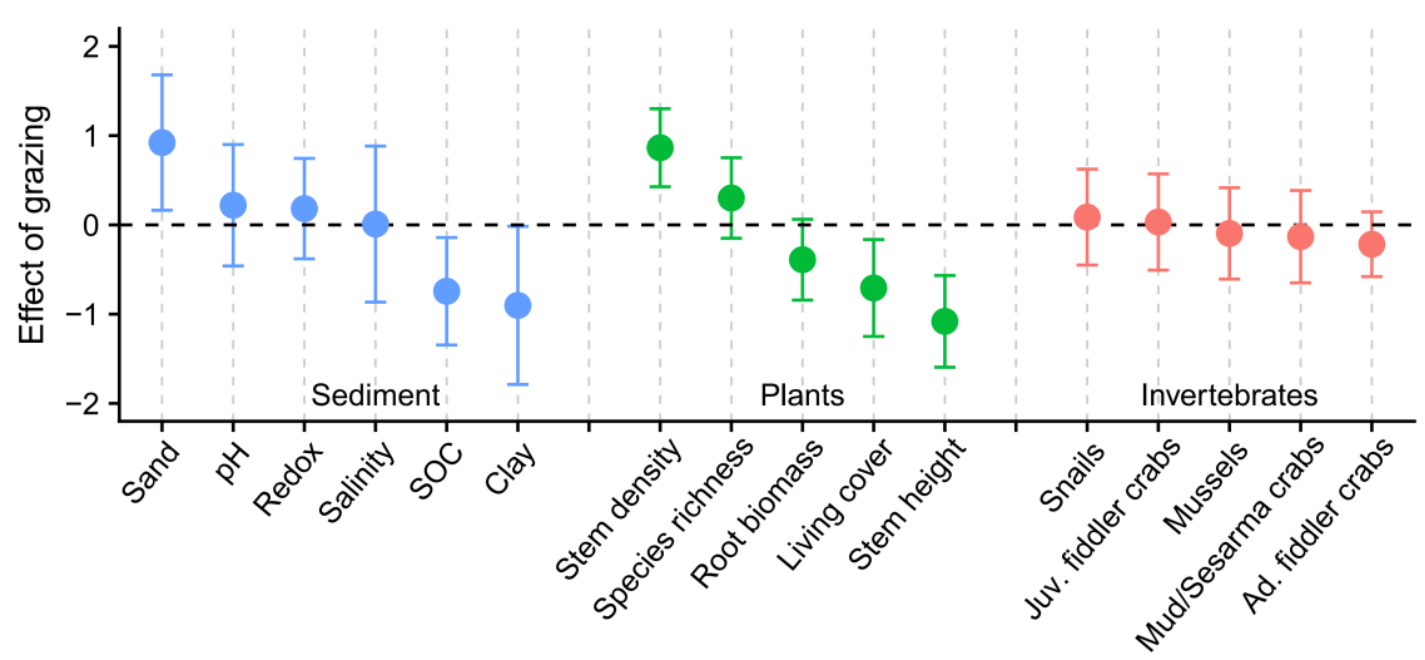

Fig. 3.3. The relationship between large mammal grazing and ecosystem properties at a landscape scale. Points show the standardised coefficients $\pm 95 \%$ confidence intervals for the effect of grazer presence relative to ungrazed control marshes. Models also controlled for season, distance from creek and latitude as fixed effects, and marsh nested within site as a random effect. SOC = soil organic carbon, Ad. = adult, Juv. = juvenile..
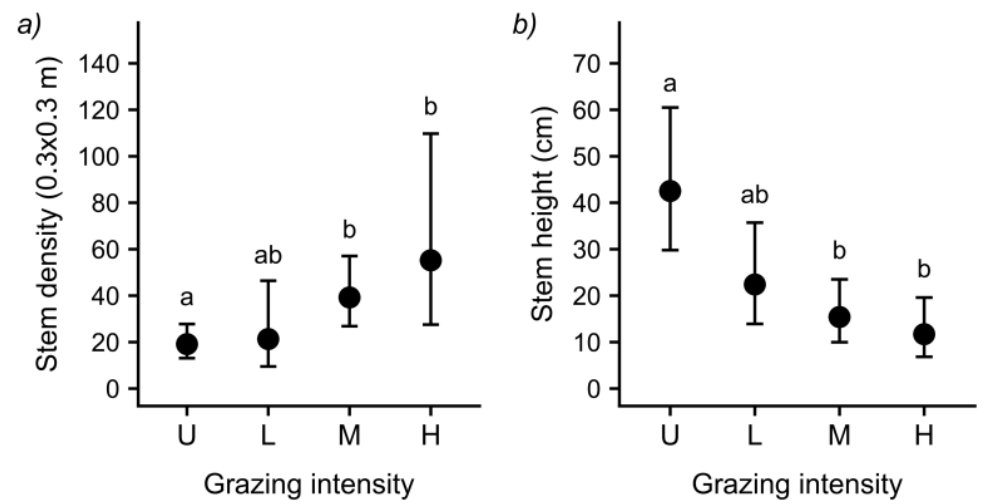

Fig. 3.4. The relationship between grazing intensity and a) stem density, and b) stem height.. Models also controlled for season, distance from creek and latitude as fixed effects, and marsh nested within site as a random effect. Points show the predicted means $\pm 95 \%$ confidence intervals (all backtransformed from the log scale) in ungrazed $(U)$, lightly $(L)$, moderately $(M)$ and heavily $(H)$ grazed marshes. Different letters indicate grazing levels that are significantly different. Grazing intensity was not significant for any other responses tested in our observational study (Table B3).

Grazing was associated with lower SOC, but also with higher sand content and lower clay content. As we cannot infer the causation of this grazing-soil texture relationship, I tested whether the effect on SOC was still present when I accounted for soil texture in the models, and whether soil texture was a better predictor of SOC than grazing. I found that grazing was consistently the best predictor out of grazing 
$(G)$, sand content $(S)$ and clay content $(C)$ - the grazing only model had a lower AIC value than any of the models incorporating soil texture (Table 3.4).

Latitude was a significant predictor of five variables and distance from the creek was a significant predictor of seven variables (Tables B2, B3). Increased latitude was associated with increases in $\mathrm{pH}, \mathrm{SOC}$, root biomass, and stem density, and decreases in adult fiddler crab densities. Increased distance from the creek was associated with increased sandiness and snail density, and decreased SOC, plant cover, stem height, adult fiddler crab density and mud/Sesarma crab density.

Table 3.4. Results of LMMs to predict soil organic matter (SOC) by grazing (grazed or ungrazed), soil sand content (\%) and soil clay content (\%). All models also had season, distance from creek and latitude as fixed effects, and marsh nested within site as a random effect. $\beta=$ standardised coefficient, Marg=marginal (i.e. fixed effects only), Cond=conditional (i.e. fixed and random effects). $\mathrm{N}=82$ for all models.

\begin{tabular}{|c|c|c|c|c|c|c|c|c|c|c|c|}
\hline Model & Response & Predictors & $x^{2}$ & $\mathbf{P}$ & $\beta$ & df & $\begin{array}{l}\text { Marg } \\
\mathbf{R}^{2}\end{array}$ & $\Delta R^{2}$ & $\begin{array}{l}\text { Cond } \\
R^{2}\end{array}$ & AIC & $\triangle \mathrm{AIC}$ \\
\hline G & (Log) SOC & Grazed & 10.60 & 0.001 & -0.64 & 8 & 0.502 & & 0.811 & 159.4 & \\
\hline Null & (Log) SOC & - & - & - & - & 7 & 0.041 & -0.461 & 0.827 & 164.3 & +4.9 \\
\hline $\mathrm{GC}$ & (Log) SOC & Grazed & 8.34 & 0.004 & -0.56 & 9 & 0.500 & -0.002 & 0.833 & 165.2 & +5.8 \\
\hline & & Clay content & 4.58 & 0.032 & 0.19 & & & & & & \\
\hline C & (Log) SOC & Clay content & 6.83 & 0.009 & 0.22 & 8 & 0.102 & -0.400 & 0.833 & 168.5 & +9.1 \\
\hline GS & (Log) SOC & $\begin{array}{l}\text { Grazed } \\
\text { Sand content }\end{array}$ & $\begin{array}{l}9.12 \\
0.39\end{array}$ & $\begin{array}{l}0.003 \\
0.533\end{array}$ & $\begin{array}{l}-0.62 \\
-0.06\end{array}$ & 9 & 0.489 & -0.013 & 0.814 & 169.9 & +10.5 \\
\hline S & (Log) SOC & Sand content & 1.86 & 0.172 & -0.11 & 8 & 0.050 & -0.452 & 0.825 & 173.6 & +14.2 \\
\hline
\end{tabular}

\subsubsection{Experimental study}

\section{Baseline tests}

At the start of the experiment, control and experimental plots did not differ in soil variables (elevation, $\mathrm{pH}$, salinity, soil organic matter), invertebrate densities (periwinkle snails, juvenile fiddler crabs, adult fiddler crabs, mud/Sesarma crabs, mussels), root biomass, plant species composition (proportion of Spartina, Salicornia, Distichlis), stem density, or hoofprint density (Table B4). The stem height in 
treatment plots (ungrazed) was significantly shorter, but only by $1.25 \pm 0.49 \mathrm{~cm}$ $(P=0.038)$. There was no significant difference between control plots and unmarked reference points at the start of the experiment for any variables (elevation, dead plant cover, stem height, stem density, hoofprint density), but on subsequent visits I found that the stem height in control grazed plots was significantly shorter (5.73 \pm $2.46 \mathrm{~cm}, \mathrm{P}=0.031$ ) than the stem height in unmarked reference points (Table B5). However, four of the experimental pairs were affected by saltmarsh die-off to varying degrees, whereas none of the reference points were. When I excluded the affected plots, there was no difference in stem height between grazed control plots and unmarked reference points. There was no difference in any other recorded variable between control grazed plots and unmarked reference points after the start of the experiment (living plant cover, dead plant cover, stem density, proportion of stems bitten, hoofprint density).

\section{Effect of grazing on ecosystem properties}

After 18 months of grazer exclusion, there were significant changes to multiple plant properties: the removal of horses led to increased stem height, living plant cover, dead plant cover, flowering stem density and Spartina cover, but reduced Salicornia cover and blue-green algae (Fig. 3.4, Table B6). However, none of the sediment properties were significantly different under different grazing treatments, and fiddler crabs were the only invertebrate that exhibited a response to grazing: adult fiddler crabs were more common in ungrazed plots, although juvenile fiddler crabs showed the opposite (but not significant) response.

For properties that were significantly different after 18 months, I investigated their responses to grazer exclusion at each time point $(0,7,13,18$ months; contingent on data availability, see Fig. B3, Table B7 for results). The increase in stem height and stem flowering was significant after just 7 months, but the increase in relative Spartina cover, decrease in relative Salicornia cover, and increase in adult fiddler crab density was not apparent until 13 months. Percentage cover of living and dead vegetation were not recorded at 7 months, but the differences were significant when 
measured at 13 months. The reduction in blue-green algae was not significant until 18 months after horse removal.

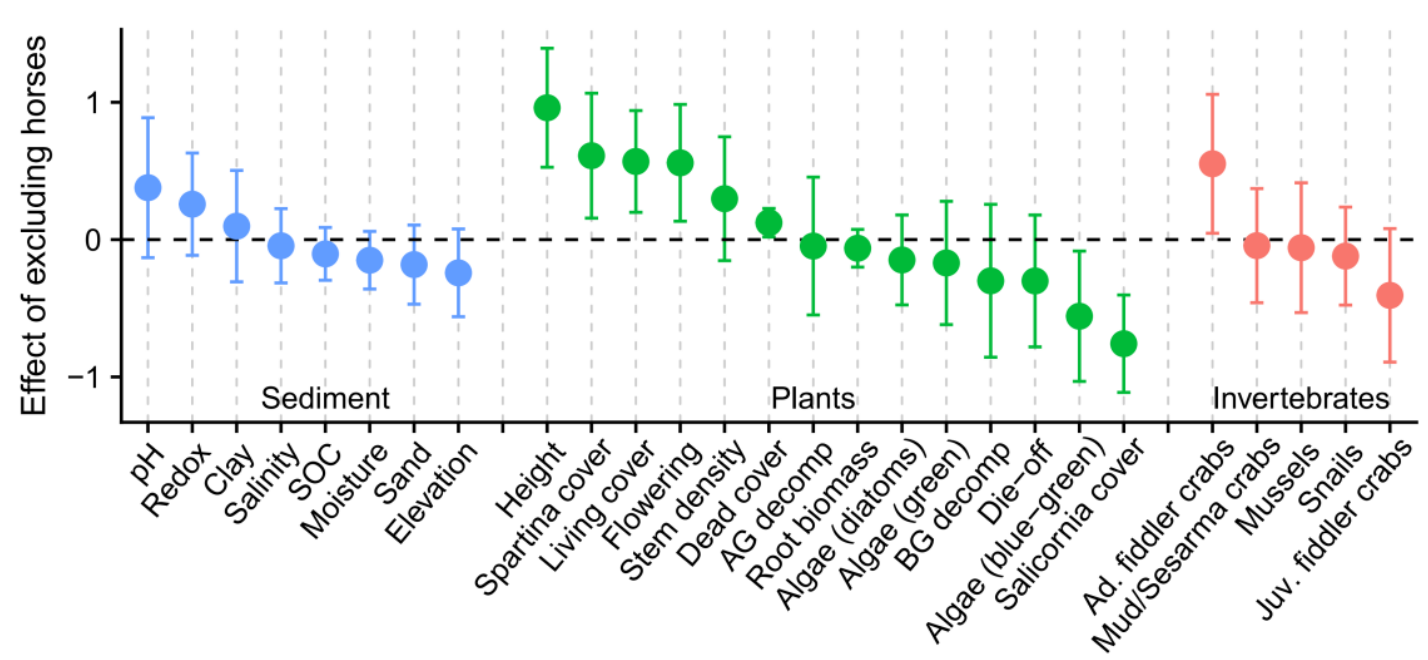

Fig. 3.5. The effect of excluding horses on ecosystem properties after 18 months. Points showed the standardised (calculated on z-transformed data) coefficients $\pm 95 \%$ confidence intervals for the effect of grazer exclusion relative to control grazed plots. Note that the direction of this effect is the opposite of Fig. 3.3, which showed the effect of horse grazing. SOC = soil organic carbon, AG decomp = aboveground decomposition, $B G$ decomp = belowground decomposition, $\mathrm{Ad}$. = adult, Juv. = juvenile.

\section{Effect of grazing on recovery}

I explored whether grazing affected the capacity of saltmarsh vegetation to recover from damage by testing the effect of the interaction between grazing and simulated hog damage on four vegetation responses (live cover, dead cover, stem height, stem density), 6 months after and 12 months after damage (Fig. 3.6, Table B8). I considered both absolute, across-grazing recovery (ungrazed damaged vs grazed damaged vegetation) and relative, within-grazing recovery (damaged $v s$ undamaged vegetation within each grazing category). Across the grazing treatments, all vegetation responses were higher in ungrazed damaged quadrats than in grazed damaged quadrats, but these differences were not significant for any response at either time point; i.e. absolute recovery does not differ between the two grazing levels. Within each grazing level, the four vegetation responses were always higher in the undamaged quadrats than in the damaged quadrats. Within the ungrazed plots, these differences were significant for every response at both 6 and 12 months, i.e. relative recovery was not achieved in ungrazed plots for any response. However, in 
the grazed plots, the difference in stem height and stem density between damaged and undamaged quadrats was no longer significant after 12 months, i.e. relative recovery was achieved for these two measures in grazed plots after 12 months.
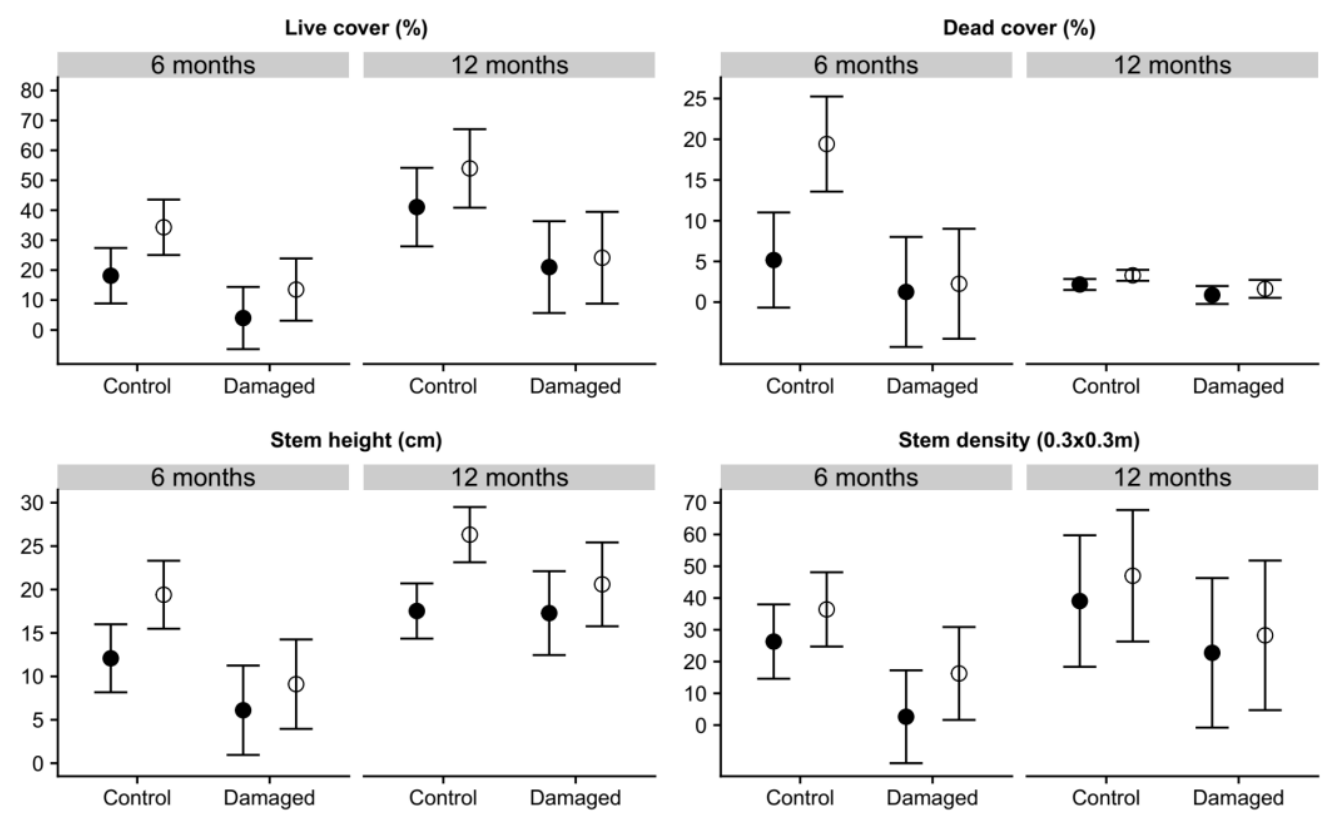

Fig. 3.6. Recovery from simulated hog damage in grazed and ungrazed plots after 6 months and after 12 months. Points show predicted means $\pm 95 \%$ confidence intervals in damaged quadrats and undamaged control quadrats positioned in grazed (filled circles) and ungrazed (open circles) plots. See Table B8 for pairwise differences between treatments.

\subsection{Discussion}

Our observational study showed that long-term large ungulate grazing affects multiple plant properties and is associated with significant differences in some sediment properties, including reductions in soil organic carbon (SOC). However, long-term grazing had no effect on snails, crabs or mussels. Our experimental study confirmed that horse exclusion has significant effects on multiple saltmarsh vegetation properties, but no changes to sediment properties were apparent after 18 months. Fiddler crabs were the only invertebrate that responded to grazer exclusion in the experimental study. Excluding horses did not increase ecosystem resilience either in terms of resistance to die-off or recovery from simulated hog damage. However, the experimental data were collected after just two growing seasons under the new grazing regime, and a longer time period may be necessary for some effects to appear. 
There were no direct contradictions (i.e. a significant positive effect vs a significant negative effect) between the results obtained in these studies and the results obtained in the global meta-analysis of Chapter 2, although some properties that displayed significant impacts of grazing in the meta-analysis (soil salinity, soil redox, plant richness) showed no significant effect of grazing in these studies (Table 3.5). However, meta-analyses are used to reveal effects where individual studies may lack power (Koricheva et al. 2013), therefore it is not surprising to see smaller or nonsignificant changes in these studies. These new studies strengthen several results obtained in the meta-analysis (a negative effect of grazing on soil carbon in America, plant height and cover), and have added data for multiple properties and processes that had insufficient data to analyse in the meta-analysis (Table 3.5).

Table 3.5. Effect of livestock grazing on properties and processes in salt marshes. Results summarised from global meta-analysis (MA, Chapter 2), long-term observational study of US salt marshes (OBS, this study) and short-term experimental study of US salt marshes (EXP, this study). Changes to properties are shown as significantly higher $(\uparrow)$, no significant effect $(-)$, or significantly lower $(\downarrow)$ with grazing. Blank spaces indicate the property was not measured. AG = aboveground, BG = belowground, ad. = adult.

\begin{tabular}{|c|c|c|c|c|}
\hline & Property/process & MA & OBS & EXP \\
\hline \multirow{7}{*}{$\overline{\bar{i}}$} & Bulk density & $\uparrow$ & & \\
\hline & Salinity & $\uparrow$ & - & - \\
\hline & Daytime temperature & $\uparrow$ & & \\
\hline & Organic carbon & $\downarrow$ (in Americas) & $\downarrow$ & - \\
\hline & $\mathrm{pH}$ & - & - & - \\
\hline & Accretion/elevation & - & & - \\
\hline & Redox & $\downarrow$ & - & - \\
\hline \multirow{7}{*}{ 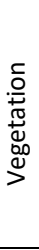 } & Species richness & $\uparrow$ & - & \\
\hline & BG biomass & - & - & - \\
\hline & Leaf nitrogen & - & & \\
\hline & Cover & $\downarrow$ & $\downarrow$ & $\downarrow$ \\
\hline & Canopy height & $\downarrow$ & $\downarrow$ & $\downarrow$ \\
\hline & AG biomass & $\downarrow$ & & \\
\hline & Litter & $\downarrow$ & & \\
\hline$\stackrel{\check{C}}{\complement}$ & Vertebrate abundance & - & & \\
\hline 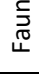 & $\begin{array}{l}\text { Invertebrate abundance } \\
\text { Invertebrate richness }\end{array}$ & $\begin{array}{c}\downarrow \text { (herbivores only) } \\
\downarrow\end{array}$ & - & $\downarrow$ (ad. fiddler crabs only) \\
\hline \multirow{13}{*}{ 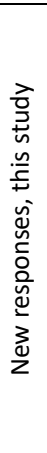 } & Sand content & & $\uparrow$ & - \\
\hline & Stem density & & $\uparrow$ & - \\
\hline & Relative Salicornia cover & & & $\uparrow$ \\
\hline & Algae (blue-green) & & & $\uparrow$ \\
\hline & Soil moisture & & & - \\
\hline & Algae (diatoms) & & & - \\
\hline & Algae (green) & & & - \\
\hline & Saltmarsh die-off & & & - \\
\hline & AG decomposition & & & - \\
\hline & BG decomposition & & & - \\
\hline & Clay content & & $\downarrow$ & - \\
\hline & Relative Spartina cover & & & $\downarrow$ \\
\hline & Flowering & & & $\downarrow$ \\
\hline
\end{tabular}


Consistent with the results from the global meta-analysis (Chapter 2), both the experimental and observational studies exhibited reduced plant biomass (stem height, percentage cover) with grazing. Reduced biomass may have consequences for ES, such as shoreline stability due to reduced accretion (Turner et al. 2002; Mudd et al. 2010; Shepard et al. 2011); storm protection due to reduced wave attenuation (Shepard et al. 2011; Möller et al. 2014; Heuner et al. 2015); fisheries support due to less detrital biomass entering the marine food chain (Bell 1997; Levin et al. 2002; MacKenzie and Dionne 2008; Baker et al. 2016); and reduced diversity and abundance of enigmatic wild species such as Clapper Rails (Rallus crepitans) and Marsh Wrens (Cistothorus palustris) which need long vegetation for nesting (Sherr 2015; Valdes et al. 2016), and manatees (Trichechus manatus) that feed in Spartina (Baugh et al. 1989).

The global meta-analysis also revealed a significant increase in plant richness with grazing (Chapter 2). The increase in plant richness in the US observational study was not significant, perhaps due to lower power in the current study, or due to the lower overall species richness in US salt marshes (Adam 1990). However, long-term grazed US marshes had significantly wider variation in plant community composition than ungrazed marshes, and the experimental study indicates that suppression of biomass and cover of the dominant species by grazers allows other plants to colonise:

Spartina cover was proportionally lower, Salicornia cover was proportionally higher, and algae concentrations were higher in grazed than ungrazed experimental plots. Often, higher plant diversity is considered a desirable outcome, and much evidence supports a link between biodiversity and the magnitude and stability of ecosystem functioning and ES (Griffin et al. 2009; Balvanera et al. 2014). However, Spartina is the dominant species in east coast US marshes (Adam 1990), so reduction in both its absolute and relative cover in grazed marshes is likely to have cascading effects on saltmarsh functioning. Spartina has very high productivity - its biomass feeds into both terrestrial and marine food chains (Teal 1962) and provides cover from predation (Levin et al. 2002); Spartina supports higher rates of soil carbon accumulation than other saltmarsh genera (Ouyang and Lee 2014); and as one of the tallest saltmarsh plants (Adam 1990), it is particularly effective for wave attenuation (Shepard et al. 2011; Narayan et al. 2016). Spartina is a foundation species that 
facilitates salt marsh establishment (Altieri et al. 2007; Dijkstra et al. 2012; Yando et al. 2019), so the reduced Spartina flower production will lead to a smaller seedbank, and may lower the capacity to establish new marshes or recolonise following disturbance such as saltmarsh die-back. When habitats are disturbed by grazing, it not only allows other native species to flourish, it can also open a niche for invasive species (Hobbs and Huenneke 1992; Bergelson et al. 1993; Belsky and Gelbard 2000). An invasive Eurasian lineage of the common reed Phragmites australis is rapidly spreading throughout US tidal wetlands, from the northern Atlantic coast to the Gulf of Mexico, and is an increasing management concern due to its impact on wetland functions and services (Chambers et al. 1999; Hazelton et al. 2014). P. australis is a disturbance specialist (Hazelton et al. 2014), therefore there is a risk that grazing disturbance could increase the invasion risk of this reed.

The long-term grazed marshes were associated with lower SOC, as well as higher sand and lower clay content in the sediments. The differences in soil texture could be due to lower rates of capture and settling of fine clay particles with the reduced aboveground biomass in grazed marshes (Mudd et al. 2010). There is some tentative evidence from the experimental study that this may be the case - horse-exclusion plots showed slightly higher clay and slightly lower sand contents, although these changes were not significant. However, the association could also be driven by grazer behaviour, as ungulates prefer drier, firmer soils (Sharps et al. 2017), so may preferentially graze on sandier sediments. SOC is generally higher in fine-textured soils (Hu et al. 2014; Bai et al. 2016; Kelleway et al. 2016) so I tested whether soil texture was driving the apparent reduction in SOC with grazing, but found that $\mathrm{SOC}$ was much better predicted by grazing than by soil texture. These data confirm and strengthen the result presented in Chapter 2 - that soil organic carbon is reduced by ungulate grazing in American salt marshes - by using standardised sampling methods across multiple sites, and controlling for important covariates (in particular, soil texture). The difference between America and Europe (where grazing does not impact soil carbon) may be due to the mechanism of soil accretion in the two systems: European marsh sediments mostly accrete by deposition of silt during tidal flooding, whereas American sediments mostly accrete by accumulation of organic matter (Bakker et al. 2015), which is strongly reduced with grazing. The difference 
may also be due to the different plant communities in the two continents. The higher diversity in European marshes (Adam 1990) may confer an increased resistance and adaptability of European saltmarsh plant communities to grazing (Callaway et al. 2005; Meirland et al. 2013), compared to Spartina-dominant US marshes. Although the experimental study did not show an effect of excluding horses on SOC, ongoing monitoring at this site may reveal changes over the longer term. However, it is likely that changes in SOC will not be as strong in the experimental site as in some of the more northerly observational sites, as SOC was generally lower at lower latitudes.

Despite the significant changes to saltmarsh vegetation with grazing, there was little observable impact on primary and secondary consumers. In the observational study there were no changes to any invertebrates with grazing, suggesting these predominantly detritivorous/herbivorous invertebrates are remarkably robust to competition and disturbance from large ungulates. This result contrasts with the significant negative effect of large ungulates on herbivorous invertebrates seen in Chapter 2. It is possible that the extremely high productivity in east coast US marshes, and restricted distribution of large grazers, means that Spartina detritus from other ungrazed areas of marsh will be redistributed and deposited in the grazed areas. Or that more frequent tidal flooding and a flexible diet allows these east coast invertebrates to incorporate both terrestrial and marine, aboveground and belowground, bacterial, plant and animal matter in their diet (Teal 1962; Alexander 1979; Coverdale et al. 2012; Soomdat et al. 2014) under competition from large herbivores. The experimental horse-exclusion plots had higher densities of adult fiddler crabs, although juvenile fiddler crabs showed the opposite (but not significant) trend. The reason for the preferences exhibited in this study is not clear. It may be related to sediment properties (grazed saltmarsh sediments are more compact, Chapter 2) or dietary preferences - adult fiddler crabs may be better able to consume the abundant Spartina biomass in ungrazed plots while juvenile fiddler crabs may prefer feeding on algae, which was higher in grazed plots. Fiddler crabs are important bioturbators, helping to oxygenate sediment and transport nutrients and organic matter, which is beneficial for saltmarsh production (Bertness 1985; McCraith et al. 2003; Daleo et al. 2007) and can ameliorate the negative effects of overgrazing by periwinkle snails (Gittman and Keller 2013). However, the contrasting 
responses of adult and juvenile fiddler crabs, and lack of response of other invertebrates, indicates that functions predicted by invertebrate biomass such as secondary production, bioturbation, and infiltration (Hensel and Silliman 2013; Angelini et al. 2015) are not affected by horse grazing. However, periwinkle snails can exert strong top-down control of Spartina biomass by direct consumption and facilitation of fungal growth (Silliman and Bertness 2002; Silliman and Newell 2003), so the fact that there are the same number of periwinkle snails grazing on a reduced Spartina biomass may mean that it becomes overstressed and more prone to die-off (Silliman et al. 2005), although there was little evidence of that in the experimental study results.

In this study, removal of grazers did not have any clear effect on saltmarsh resilience. Grazed plots were not significantly more susceptible to die-off than ungrazed plots and recovery from simulated hog damage was not improved by excluding horses. One year after damage, stem height and density in damaged grazed quadrats had recovered to the level of the undamaged grazed quadrats. However, damaged ungrazed quadrats had not recovered to the same level as the undamaged ungrazed quadrats for any of the four vegetation measures. Therefore, relative recovery was quicker in grazed plots than in ungrazed plots. The ungrazed plots had a higher recovery benchmark than the grazed plots, but even in terms of absolute recovery, ungrazed damaged plots were not significantly better than grazed damaged plots. Chapter 2 provides evidence that grazed saltmarsh plants have greater root biomass, which could facilitate recovery following other forms of damage. However, that result was dominated by European studies, and was not evident in the US experimental study (no change in root biomass) and contradicted by the US observational study (non-significant reduction in root biomass). An alternative explanation is that reduced resilience of Spartina in grazed plots may be compensated by increased cover of Salicornia, which is less susceptible to die-off (National Park Service 2019) and, as a sprawling, shrubby plant, may be more resistant to hog damage. However, these results follow just 1.5 years of grazer exclusion, therefore it is too early to reach conclusions about how long-term grazing/absence of grazing may affect saltmarsh resilience. There may be additional trade-offs from large ungulate grazing that are outside the scope of this study, such 
as increased faecal bacteria in marine waters, potentially affecting fisheries and bathing water quality (Kershaw et al. 2012; Devane and Gilpin 2015).

\subsection{Conclusions and management implications}

The observational surveys build on the results from Chapter 2, providing strong evidence that grazing of livestock on American salt marshes trades-off against their blue carbon value. However, recovery of soil carbon is not yet evident after 1.5 years of experimental grazer exclusion in a Georgia salt marsh.

Grazing affects multiple plant properties, but has little apparent cascading effect on consumers or ecosystem resilience, although additional effects may emerge over the longer term in the experimental study. Plant biomass contributes to various important functions and services. Reductions in biomass and Spartina dominance may negatively affect the marine life that depends on saltmarsh vegetation for food and shelter, birds that nest in marshes, saltmarsh accretion rates, and wave attenuation capacity. Disturbance by grazers may also increase the risk of invasion from non-native species such as Phragmites australis.

Despite these significant local effects, ungulate grazing is unlikely to have a large effect at the landscape scale along the US east coast under current grazing practices: salt marshes cover a large area along this coast, and only a small proportion are grazed by horses or livestock. With the exception of Cumberland Island, all horsegrazed barrier islands now manage their horse populations, to keep within sustainable numbers. Even on Cumberland, horses have access to only a small proportion of the total area of salt marsh, and do not appear to significantly affect multifunctionality or resilience in the areas they do graze. Horses also provide benefits in the form of enhanced tourism as well as less tangible cultural services such as symbolic and aesthetic values, and community identity (Gruenberg 2015). In other regions, such as South America, where livestock grazing of Spartina marshes is much more common (Costa et al. 2009), grazing is likely to have more important, landscape-scale effects on blue carbon, and possibly other ES. However, east coast US salt marshes are more under threat from land development, pollution, climate 
change, and runaway consumer control through over-harvesting of predators (Gedan et al. 2009). The strongest argument to reduce the horse population on Cumberland Island may be humane reasons, due to horse welfare (Gruenberg 2015) rather than ecological reasons. However, livestock grazing should certainly not be widely practiced along the US east coast: recommendations that formerly-reclaimed marshes can be restored and grazed, without harming carbon-storage, leading to win-win situations (Yu and Chmura 2010) do not apply in these marshes.

\subsection{Acknowledgements}

Many thanks to Sean Sharp, Kevin James, Rebecca Atkins, Katie Dixon, Lynsey Neilan, and Liz Shriver for their help with fieldwork and lab work; to Doug Hoffman for all his assistance with the Cumberland Island experiment; and to Brandon Puckett (NCDENR), Kevin Holscomb (Chincoteague NWR), Bill Hulslander (Assateague Island NS), Joseph Colbert and Ben Carswell (Jekyll Island State Park), and all other Park staff who facilitated observational surveys.

\subsection{Supporting Information}

The following supplementary material can be found in Appendix B:

Table B1. Visual indicators used to allocate qualitative grazing levels

Table B2. Effect of grazing on ecosystem properties in observational survey

Table B3. Effect of grazing intensity on ecosystem properties in observational survey

Table B4. Baseline comparisons, control vs experimental plots

Table B5. Baseline comparisons, control vs reference points

Table B6. Effect of grazing on ecosystem properties after 18 months

Table B7. Effect of grazing on ecosystem properties at each time point

Table B8. Effect of grazing on ecosystem multifunctionality

Table B9. Effect of grazing on recovery from damage

Figure B1. Soil texture classes triangle

Figure B2. Raw species data for observational survey marshes 
Chapter 3: Grazing in US marshes

Figure B3. Changes over time 


\section{Chapter 4: Grazing suppresses flowering of key species to reduce bee abundance and diversity on salt marshes}

Kate E Davidson, MS Fowler, MW Skov, D Forman, J Alison, M Botham, N Beaumont, JN Griffin

\subsection{Abstract}

Global declines in pollinator populations and associated services make it imperative to identify and sensitively manage valuable habitats. Coastal habitats such as salt marshes can support extensive flowering meadows, but their importance for pollinators, and how this varies with land-use intensity, is poorly understood. We hypothesised that salt marshes provide important bee foraging habitat, and that livestock grazing either suppresses or enhances its value by reducing the abundance or increasing the diversity - of flowering plants. To test these hypotheses, we surveyed 11 salt marshes in Wales (UK) under varying grazing management (longterm ungrazed, extensively grazed, intensively grazed) over three summers and investigated causal pathways linking grazing intensity with bee abundance and diversity using a series of linear mixed models. We also compared observed bee abundances to 11 common terrestrial habitats using national survey data.

Grazing reduced bee abundance and richness via reductions in the flower cover of the two key food plants: sea aster Tripolium pannonicum and sea lavender Limonium spp. Grazing also increased flowering plant richness, but the positive effects of flower richness did not compensate for the negative effects of reduced flower cover on bees. Bee abundances were approximately halved in extensively grazed marshes 
(relative to ungrazed) and halved again in intensively grazed marshes. Saltmarsh flowers were primarily visited by honeybees Apis mellifera and bumblebees Bombus spp. in mid and late summer. Compared to other broad habitat types in Wales, ungrazed salt marshes ranked highly for honeybees and bumblebees in July-August, but were relatively unimportant for solitary bees. Intensively grazed salt marshes were amongst the least valuable habitats for all bee types.

Under appropriate grazing management, salt marshes provide a valuable and previously overlooked foraging habitat for bees. The strong effects of livestock grazing identified here are likely to extend geographically given that both livestock grazing and key grazing-sensitive plants are widespread in European salt marshes. We recommend that long-term ungrazed salt marshes are protected from grazing, and that grazing is maintained at extensive levels on grazed marshes. In this way, salt marshes can provide forage for wild and managed bee populations and support ecosystem services.

\subsection{Introduction}

Flower-visiting insects, particularly bees, provide a vital ecosystem service by pollinating crop plants and wild flowers (Gallai et al. 2009; Garibaldi et al. 2011; Hanley et al. 2015). However, pollinators are under threat from habitat loss, agrochemicals, disease, invasive species and climate change (Brown and Paxton 2009; Potts et al. 2010a), leading to long-term declines in wild and managed pollinator populations (Potts et al. 2010a; Potts et al. 2010b; Powney et al. 2019). As a result of pollinator declines, international, national and regional governing bodies have introduced pollinator strategies to promote pollinator conservation and the provision of pollinator-friendly habitat (e.g. the EU Pollinators Initiative 2018, the AllIreland Pollinator Plan 2015-2020, the Action Plan for Pollinators in Wales 2013). Agricultural intensification is a major driver of bee declines (Le Féon et al. 2010; Potts et al. 2010a), but appropriate grassland management can yield significant benefits for pollinators (Bruppacher et al. 2016; Garrido et al. 2019). However, the relationship between livestock grazing and pollinator abundance and diversity is not clear or consistent, showing positive (Vulliamy et al. 2006), negative (Kruess and 
Tscharntke 2002; Kimoto et al. 2012) and non-linear relationships (Lázaro et al. 2016; Mu et al. 2016). Difficulty in disentangling the various effects may originate from a lack of understanding of the mechanisms by which grazers influence pollinator activities and abundances in different habitats. Due to the pressing ecological need, and policy drive, to enhance pollinator-friendly habitat cover and quality, it is worth exploring less-studied habitats for their potential value (e.g. Baldock et al. 2015) and how this may alter under different management.

Salt marshes are productive grasslands of halophytic herbs, grasses and shrubs that form in the intertidal zone of sheltered coastal areas. Salt marshes deliver important ecosystem services (Barbier et al. 2011; McKinley et al. 2018), yet are rarely considered for their potential value as a pollinator habitat (Rickert et al. 2012; van Klink et al. 2016; Rickert et al. 2018). However, salt marshes can be floristically rich (Adam 1990), harbouring many flowering plants visited by bees and other pollinators (Agassiz 2000; Falk and Lewington 2015). European salt marshes are commonly grazed by livestock, with consequences for their ecosystem properties and service provision (Chapter 2: Davidson et al. 2017; Pagès et al. 2018). Although European salt marshes do not cover a large area - approximately 440,000 ha in mainland Europe and the British Isles (McOwen et al. 2017) - they sit within a wider network of seminatural coastal habitats, and could act as a vital corridor, increasing ecosystem connectivity and facilitating biological flow between crops and bee nesting and foraging areas (Viana et al. 2012). It is therefore worthwhile to understand how valuable marshes are for pollinators, and how grazing management affects their value.

Little is known about the effect of grazing on saltmarsh pollinator communities. Grazing increases saltmarsh plant richness (Chapter 2) which often predicts increased pollinator abundance and diversity (Potts et al. 2004; Vulliamy et al. 2006; Lázaro et al. 2016). However, in Wadden Sea marshes, intensive grazing led to reductions in flower and pollinator abundance compared to less intensive grazing (van Klink et al. 2016) and intensive sheep grazing disrupted moth-plant associations (Rickert et al. 2018). Additionally, livestock alter saltmarsh soil properties and reduce vegetation cover (Chapter 2) which may affect the suitability of the habitat as a nesting site for 
bees (Wuellner 1999; Vulliamy et al. 2006). To understand how and why livestock grazing impacts pollinators, we must understand the nature and strength of these potentially opposing effects.

Here, we investigate the effects of grazing on bee communities across multiple salt marshes on the south coast of Wales (UK). Marshes in this region hold plant communities typical of those in north and western European sites, with a relatively high diversity of halophytic herbs (Adam 1990), and a long history of livestock grazing. We use field surveys of bee and plant communities to investigate the causal pathways linking bee abundance and diversity with grazing. We hypothesised that grazing affects bees via three pathways (Fig. 4.1a): (1) by altering the quantity of floral resource available, (2) by altering plant diversity, and (3) by modifying substrate conditions and directly disturbing bees or their nests; the net effect of grazing on bee communities depends on the balance of these positive and negative pathways. We further developed this conceptual model and incorporated covariates (weather, timing, landscape) (Fig. 4. 1b), before investigating the individual hypothesised pathways using linear mixed models. To scale up effects across the broader coastal landscape, we investigated the effect of grazing on the $\beta$-diversity of bees using multivariate analysis of pairwise community distance matrices. Finally, we assessed how saltmarsh bee abundances compare to various terrestrial habitats, using pollinator survey data collected across Wales for the Glastir Monitoring and Evaluation Programme (Emmett and the GMEP team 2017). To the best of our knowledge, this is the first study to compare bee communities in grazed and ungrazed marshes, and to quantify the importance of salt marshes as a bee foraging habitat, relative to terrestrial habitats.

\subsection{Methods}

\subsubsection{Field surveys}

We surveyed 11 salt marshes in south Wales (UK) every summer from 2016-2018 (Fig. 4.2, see Appendix C Table C1 for full site details). Sites were selected to cover a range of grazing intensities (4 long-term ungrazed, 3 extensive, 4 intensive) and livestock species (cattle, ponies, sheep), and to be large enough to allow sampling 
a)

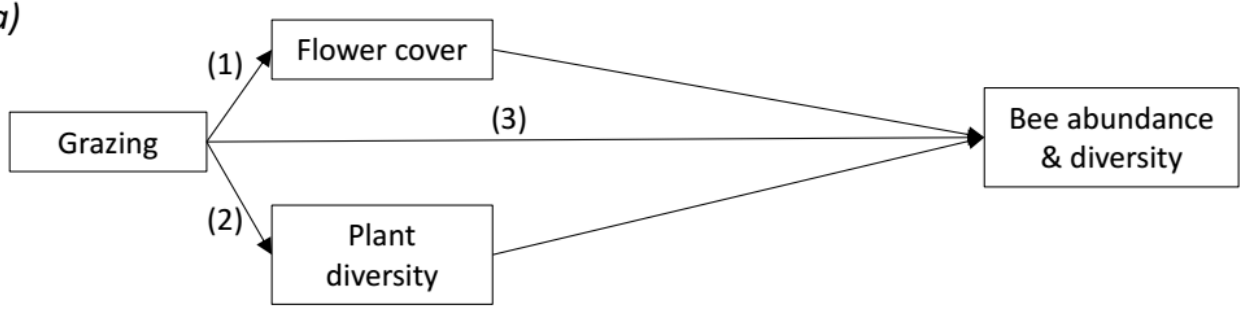

b)

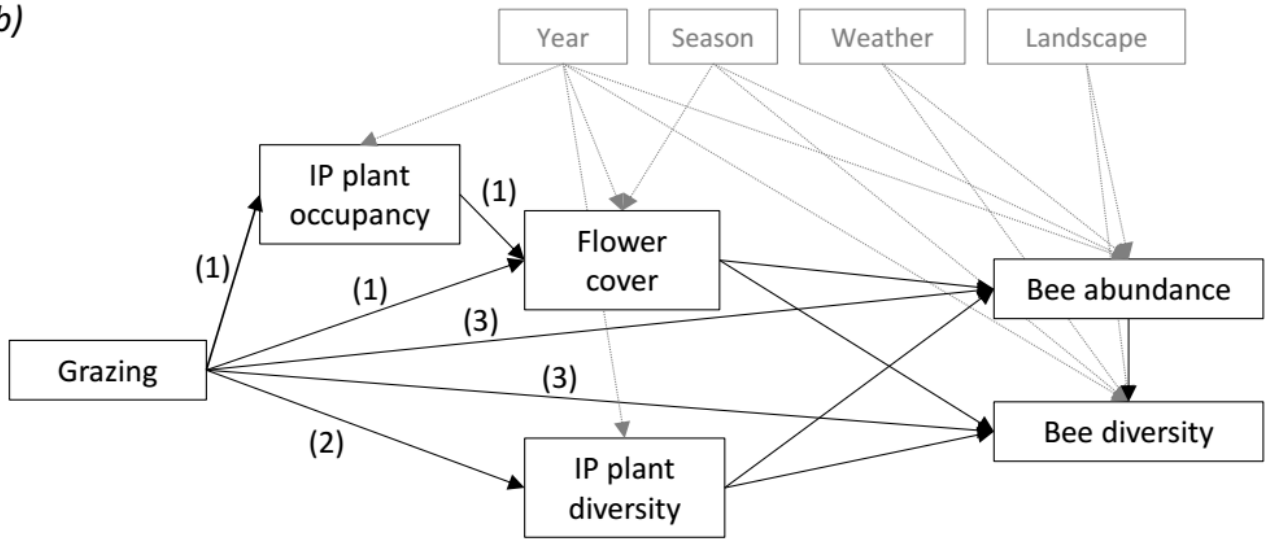

Fig. 4.1. Mechanisms by which grazing may affect bee communities. a) Simplified system showing three hypothesised pathways: (1) changes to insect-pollinated (IP) plant flower cover; (2) changes to IP plant diversity, (3) direct disturbance of bees or their nests. $b$ ) Theoretical system to be tested using linear mixed models, allowing pathway (1) to operate via changes to IP plant cover (occupancy) and direct changes to flower cover via defoliation. Temporal and environmental variables in grey will be included in models to control for these effects.

along four $200 \mathrm{~m}$ transects covering multiple inundation zones and vegetation communities. It was not possible to calculate accurate grazing levels in livestock units per hectare (LUha $\left.{ }^{-1}\right)$, because several of our study sites were part of much larger unfenced grazing lands with extremely clustered livestock distribution. Grazing categories (absent, extensive, intensive) were evaluated based on dung counts and visual assessments performed at each transect on every visit, then averaged for the study site as a whole (see Appendix C Supplementary methods). Ungrazed marshes had not been grazed by livestock for a minimum for 30 years. Extensively grazed marshes were characterised by having little damage to standing biomass and turf, and a relatively complex sward (estimated grazing density of $0.3-0.4$ LUha $^{-1}$ during the summer grazing period). Intensively grazed marshes were characterised by removal of much of the standing biomass, leaving a relatively short, uniform sward, and widespread compaction or cutting up of the turf by animal trampling (estimated grazing density $>0.8$ LUha $^{-1}$ ). Variation in sediment type, marsh geomorphology and 
surrounding landscape was distributed evenly across the grazing categories (Table C1).

Each marsh was surveyed along four $200 \mathrm{~m}$ fixed transects on seven days over three summers, covering the major flowering period in these marshes. Marshes were surveyed in mid-summer (12th July - 10th August) in 2016, and in early (9th - 29th June), mid (12th July - 10th August) and late (24th August - 19th September) summer in 2017 and 2018. Surveys were conducted between 10am and 5pm, preferably on warm, calm, dry days (minimum temperature: $15^{\circ} \mathrm{C}$, maximum wind speed: $7.5 \mathrm{~m} \mathrm{~s}^{-1}$ ). Transects were run parallel to the shore and distributed as evenly as possible across each marsh (dependent upon safe access and avoidance of major creeks), to represent the major vegetation communities present.

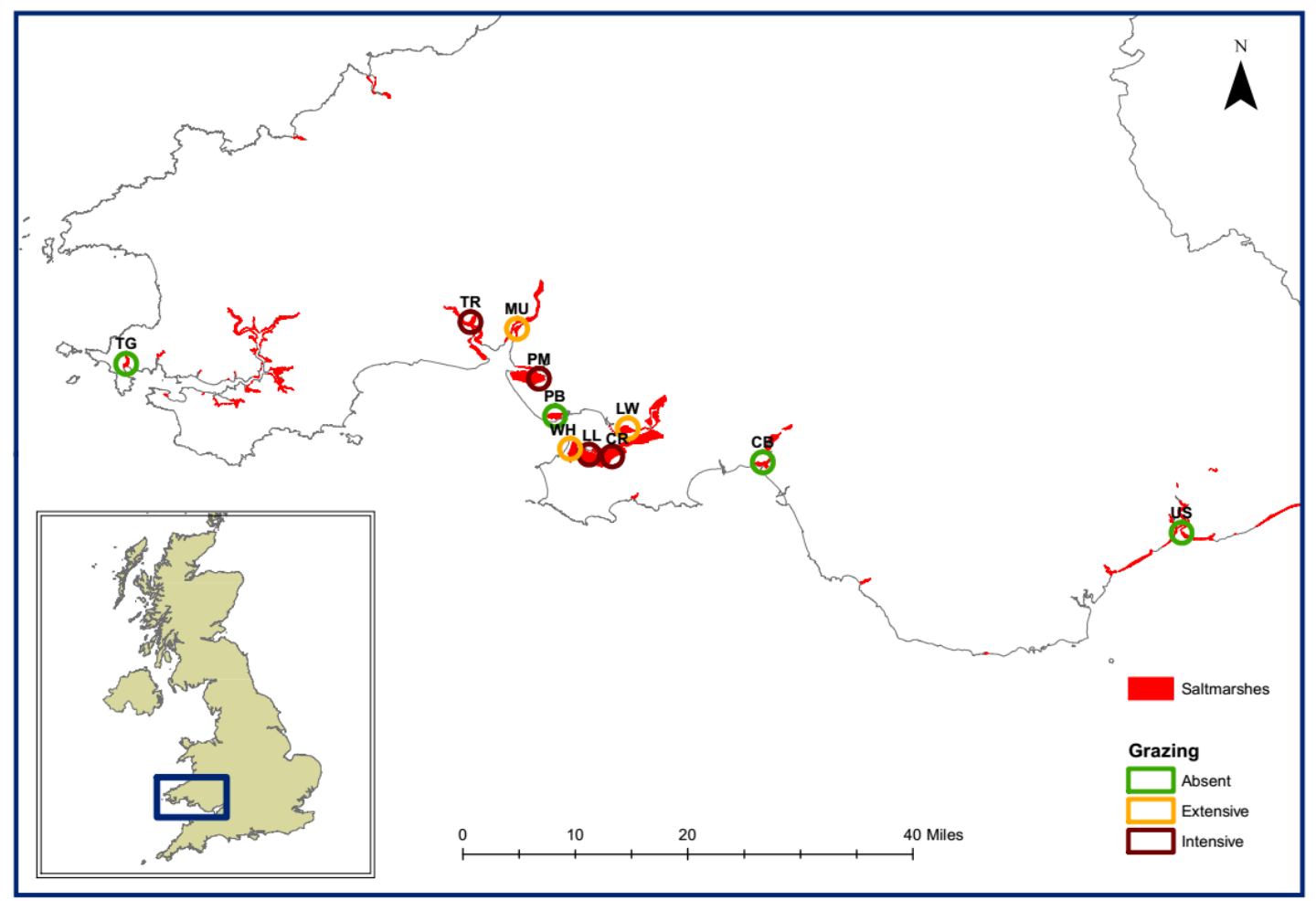

Fig. 4.2. Location of 11 study sites across south Wales, UK. See Table C1 for details of each site. The ungrazed marshes were more widely distributed geographically due to the rarity of long-term ungrazed sites. Three sites (WH, LL, CR) are situated on a single contiguous area of salt marsh $\sim 10 \mathrm{~km}$ long: each site was separated by at least $2.5 \mathrm{~km}$ and two major creek channels. However, two of these three marshes were under the same grazing management (intensive). As this could be deemed pseudoreplication, we repeated our net grazing effect models (described in section 4.3.2) with each of these sites excluded, which did not alter results. 
Bee surveys were conducted using standardised pollinator transect methodologies (Pollard and Yates 1993). The surveyor walked each 200m transect at a slow, steady pace and noted any bee visiting a flower within a $5 \times 5 \mathrm{~m}$ area extending either side and in front. The flower species was noted and bees were identified to species level (with the exception of Bombus terrestris/lucorum workers, which cannot be reliably separated morphologically) using Falk and Lewington (2015). Bees that could not be identified in the field were captured in a net (a subsample of 5-10 individuals were collected when that bee type was abundant), transferred to a plastic vial with ethyl acetate, and subsequently identified under a x30 stereo microscope at Swansea University. Each transect was walked twice, with a 10 minute gap between walks to allow bees to re-settle (Baldock et al. 2015). We used the total number of bees per marsh per survey visit (i.e. bees per $1.6 \mathrm{~km}$ of transect) in our regression models.

We assessed the vegetation within a $1 \times 1 \mathrm{~m}$ quadrat placed every $20 \mathrm{~m}$ along each transect. For each plant species, we took the mean number of quadrats in which it was present to obtain an average transect occupancy for each marsh (potential range of $0-11$ ). Species composition (present/absent in quadrat) was recorded in mid-summer in 2016 and 2017. The mean for each marsh over these two years was used as a predictor in regression models. Saltmarsh plant communities in south Wales, and more widely, are relatively stable over time under consistent grazing management (Taubert and Murphy 2012; Pauls 2017), therefore we expect these mean values to be a fair reflection of the plant community across the three years. Percentage flower cover of each species and vegetation canopy height were recorded on every survey visit. Limonium vulgare and Limonium humile are grouped together in the analyses, as these species are difficult to distinguish morphologically and can hybridise (Dawson and Ingrouille 1995). We used the mean flower cover per species across all 44 quadrats in the marsh (for each visit) in our regression models.

\subsubsection{Data analysis}

For our analyses, we were only interested in plants that provide pollen and nectar resources for pollinators. Hereafter, when we refer to plants or flower cover, we only include insect-pollinated (IP) plants. Spartina grasses are generally considered to be 
wind-pollinated, but we noted several bees visiting S. anglica during our surveys, so included it with the IP plants in our analyses. All statistical analyses were conducted in R Studio running R3.5.2. (R Core Team 2018) using packages as detailed in the Supplementary methods (Appendix C).

Testing the effect of grazing on bee abundance and $\alpha$-diversity We used Linear Mixed Models (LMMs) to test the net effect of grazing on bee abundance, bee species richness, and bee Shannon diversity. We tested the effect of grazing at the marsh-scale rather than the transect-scale, as this is the relevant scale for managers. Each row in our data was a single survey visit to a marsh $(n=76)$. Each model also included year and season as predictors to explore temporal patterns of bee foraging. We included wind speed, temperature and surrounding landscape (proportion of natural habitat, see Supplementary methods for calculation) where their inclusion improved model fit (reduced AIC by $>2$ ). Marsh was included as a random effect, to control for repeated sampling within each marsh. Model assumptions were verified by examining residual plots (scaled residuals versus predicted values and versus each covariate in the model) and testing goodness of fit (Kologorov-Smirnov) of observed versus expected values. Response variables were log-transformed where necessary to meet model assumptions (Ives 2015). We used likelihood ratio tests (LRTs) using maximum likelihood (ML) estimation to test the significance of grazing, year and season. If significant, we compared different levels of these factors using t-tests. Initial and final model terms are presented in the full statistical results provided in Appendix C (Table C6).

To explore the mechanisms of grazing effects, we tested the links proposed in our hypothesised network (Fig. 4.1b). For each stage in the network, we built our initial model containing all biologically relevant predictors (see Table C7 for full details of models), with marsh as a random effect. We used AIC to determine if fit was improved by log-transformation of continuous predictors. We used LRTs to test: (1) if IP plant diversity (richness) was affected by grazing; (2) if IP plant occupancy was affected by grazing; (3) if flower cover was affected by grazing, controlling for IP plant occupancy; (4) if bee abundance was affected by grazing, IP plant richness, and 
flower cover; (5) if bee diversity (richness) was affected by grazing, IP plant richness, and flower cover, controlling for bee abundance. Two plants, sea aster Tripolium pannonicum (formerly Aster tripolium, hereafter 'Aster') and the sea lavenders Limonium spp. (hereafter 'Limonium') received 95\% of all bee visitations. To gain further insight, we repeated models (2-5) above with IP plant occupancy and flower cover separated into i) Aster, ii) Limonium, and iii) other IP plants (network presented in Fig. (2). We repeated all models using Shannon's $H^{\prime}$ as our measure of diversity. We also repeated our models with Apis mellifera excluded, to test if results are consistent for wild bees only (see Table C8 for full model details).

Plant diversity and plant occupancy were not measured in 2018, therefore models (1) and (2) test only 2016/7 data (when plant diversity and plant occupancy were used as predictors in models (3)-(5) we used the average value from $2016 / 7$ for each marsh). In south Wales, Aster blooms in late summer and Limonium blooms in midsummer. Therefore, the models to predict Aster flower cover (3.i) and Limonium flower cover (3.ii) only include late and mid-summer survey results respectively to avoid zero-inflated distributions. Sub-setting our dataset in this way precluded analysis using a structural equation modelling approach. Instead, we conducted separate LMMs for each stage of the network and have presented the results for these in a single network diagram, to allow analysis of the relative importance of each pathway.

\section{Testing the effect of grazing on B-diversity}

We explored how grazing affected three components of $\beta$-diversity (Baselga 2010) based on respective distance matrices of bee composition across sites: total $\beta$ diversity (Sørensen index, $\beta_{\text {sor }}$ ), the turnover component of $\beta$-diversity (Simpson index, $\left.\beta_{\text {sim }}\right)$ and the nestedness component of $\beta$-diversity $\left(\beta_{\text {nes }}=\beta_{\text {sor }}-\beta_{\text {sim }}\right)$. Based on these matrices, we used Permutational Multivariate Analysis of Variance (PERMANOVA, Anderson, 2001) to test whether grazing was a significant source of variation in species composition. As the PERMANOVA test is sensitive to differences in group dispersions, we also ran a permutation-based test for homogeneity of multivariate dispersions (PERMDISP, Anderson, 2006). 


\section{Comparing salt marshes with terrestrial habitats}

We compared our saltmarsh transect counts with transect counts from other habitats by integrating our July-August saltmarsh survey data with pollinator survey data collected in July-August 2013-2016 in all terrestrial broad habitat types (classified as JNCC 2019) for the Welsh Government under the Glastir Monitoring \& Evaluation Programme (Emmett and the GMEP team 2017). These data were collected from $1 \mathrm{~km}$ squares across Wales under a stratified random sampling design. Each $1 \mathrm{~km}$ square was visited in July and August during one year of the period 20132016 and surveyed for bees along ten 200m transect sections (see Supplementary methods for full details). The surveys noted all bees present on the transect, compared with only foraging bees in the saltmarsh surveys, therefore comparisons are likely to be conservative towards saltmarsh abundances. We used negative binomial mixed effects models to predict the number of honeybees, bumblebees, and solitary bees in each of 14 habitat types (11 main terrestrial habitats, ungrazed, extensively, and intensively grazed salt marsh) while controlling for the fixed effects of wind and temperature and the random effects of observer and transect nested in site. We tested whether habitat was a significant predictor of bee counts using LRTs. We were unable to control for the effect of year in our models, as year covaried with habitat type. However, each survey block (terrestrial, salt marsh) spanned $\geq 3$ years, which should minimise any effect of year.

\subsection{Results}

In total we recorded 1594 foraging bees across the 11 sites, averaging $13.1 \pm 4.0$ (S.E.) bees per km of transect, and comprising 19 species across 10 genera (see Tables C4-C5 and Figs C3-C4 for bee species data, flower cover and flower visitation data). Most individuals were either honeybees (52\%) or bumblebees Bombus spp. (47\%). We observed bees foraging on just nine plant species, although we recorded 17 species of insect-pollinated plants in the marshes. Two plants in particular, Aster (Tripolium pannonicum) and Limonium (L. vulgare, L. humile), received the great majority of bee visits ( 52 and $43 \%$ of observed visits, respectively) and were 
disproportionately preferred relative to their flower cover $(33 \%$ and $30 \%$ of total flower cover).

\subsubsection{Grazing, bee abundance and $\alpha$-diversity}

Grazing intensity had a significant effect on bee abundance and bee richness, but not on Shannon ( $\mathrm{H}^{\prime}$ ) diversity (Fig. 4.3a-c, Table $\mathrm{C6}$ ). Intensively grazed marshes had significantly lower bee abundance and bee richness than ungrazed marshes, while extensively grazed marshes were intermediate and not significantly different from the other two categories. Bee abundance, richness and $\mathrm{H}^{\prime}$ diversity were significantly higher in mid and late summer, compared to early summer (Fig. $4.3 d-f$ ) but were unaffected by survey year (Table C6).
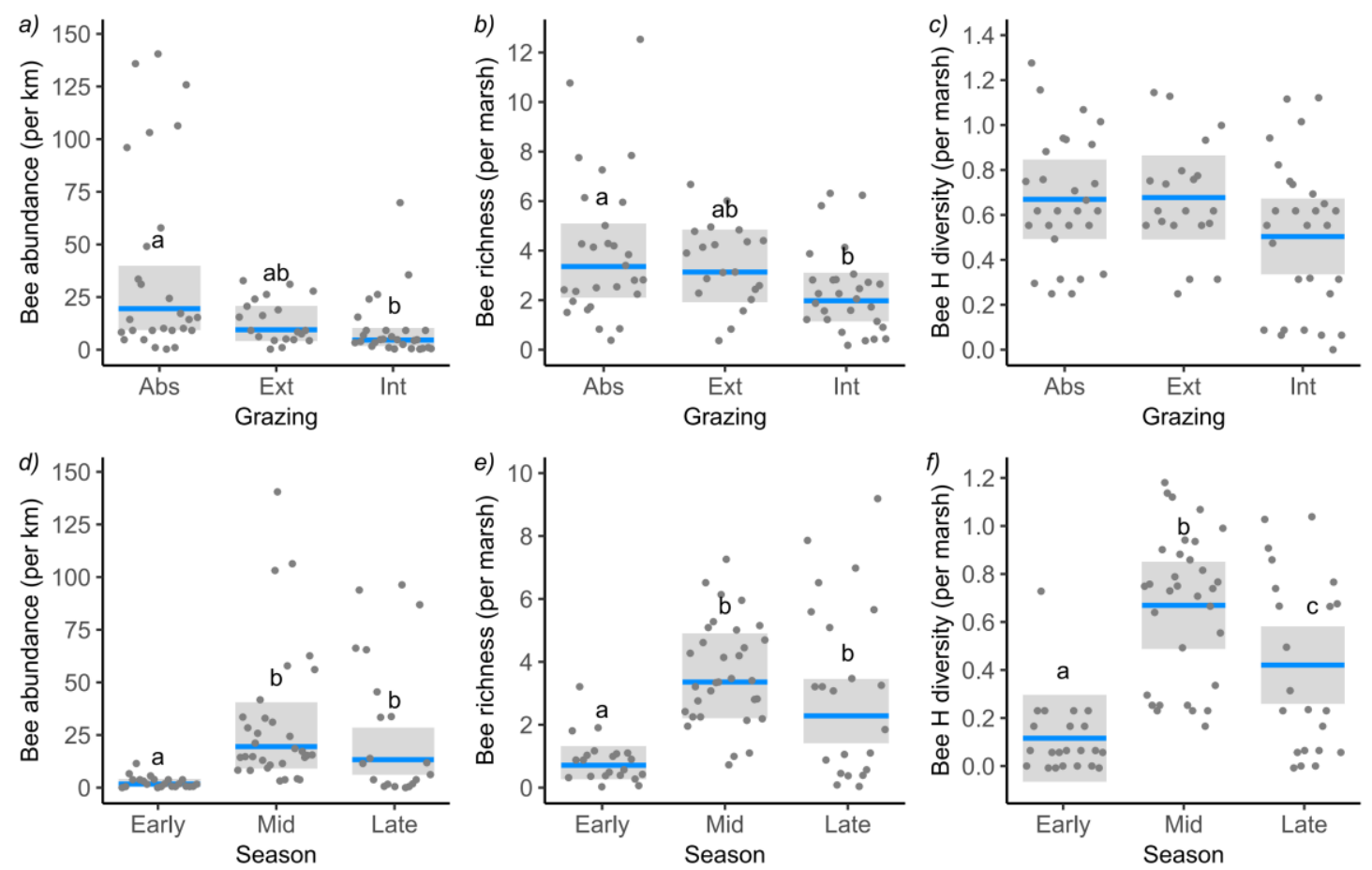

Fig. 4.3. Model predictions (blue lines) and partial residuals (points) for net grazing effects ( $a-c$ ) and seasonal effects $(d-f)$ on bees. Means are predicted for mid-summer, 2017 in $(a-c)$ and for ungrazed marshes, 2017 in $(d-f)$. Factor levels that are significantly different (t-test) are indicated by different letters. $95 \%$ confidence intervals (shaded boxes), calculated by bootstrapping. Abundance and richness values were calculated on the log scale, but have been back-transformed for presentation. 
Grazing affected bee communities via two pathways: negative effects on flower cover and positive effects on plant richness. When all plant species are combined (Fig. 4.4a, Table C7), intensive grazing reduced flower cover, which had negative effects on bee abundance, leading to reduced bee richness. However, this negative effect on bee richness was mitigated by a positive effect of extensive and intensive grazing on plant richness, and a positive effect of plant richness on bee richness.

Separating out key plant species (Fig. 4.4b, Table C8) highlights how grazing predominantly affected the flower cover of Aster and Limonium (Fig. B.1), and these plants had the strongest effects on bee abundance and therefore richness (Fig. B.2). Grazing reduced both the occupancy (i.e. coverage across the marsh) and flower cover of Limonium. Grazing had no effect on Aster occupancy, but increased grazing intensity directly reduced Aster flower cover. Grazing had no effect on other plant occupancy or flower cover. Increasing both Limonium and Aster flower cover strongly increased bee abundance. While flower cover of other plants had no significant effect, plant richness had a positive effect on bee abundance. Bee richness was predominantly affected by bee abundance, although there was a small negative effect of Aster flower cover on bee richness. When analysed with key plant species separated, the positive effect of plant richness on bee richness was no longer significant ( $P=0.056$, Fig. $\mathrm{C} 6 e$ ). When honeybees were excluded from the models, the effect of Aster cover on bee richness became positive, but otherwise results for wild bees did not differ from the models including honeybees (Table C8). When Shannon's $\left(\mathrm{H}^{\prime}\right)$ index was used as the diversity measure instead of species richness, all trends remained the same, although some predictors were no longer significant (Table C8 and Figs $\mathrm{C} 5-\mathrm{C} 6$ ).

\subsubsection{Grazing and $\beta$-diversity}

We observed 16 bee species in ungrazed marshes (eight of these being unique to ungrazed marshes, Fig. 4.5), 10 species with extensive grazing (one unique), and six species with intensive grazing (one unique). Total $\beta$-diversity (pairwise Sørensen dissimilarity) did not differ between grazing levels, either in terms of centroid location in multivariate space (PERMANOVA), or in terms of within-group dispersion 

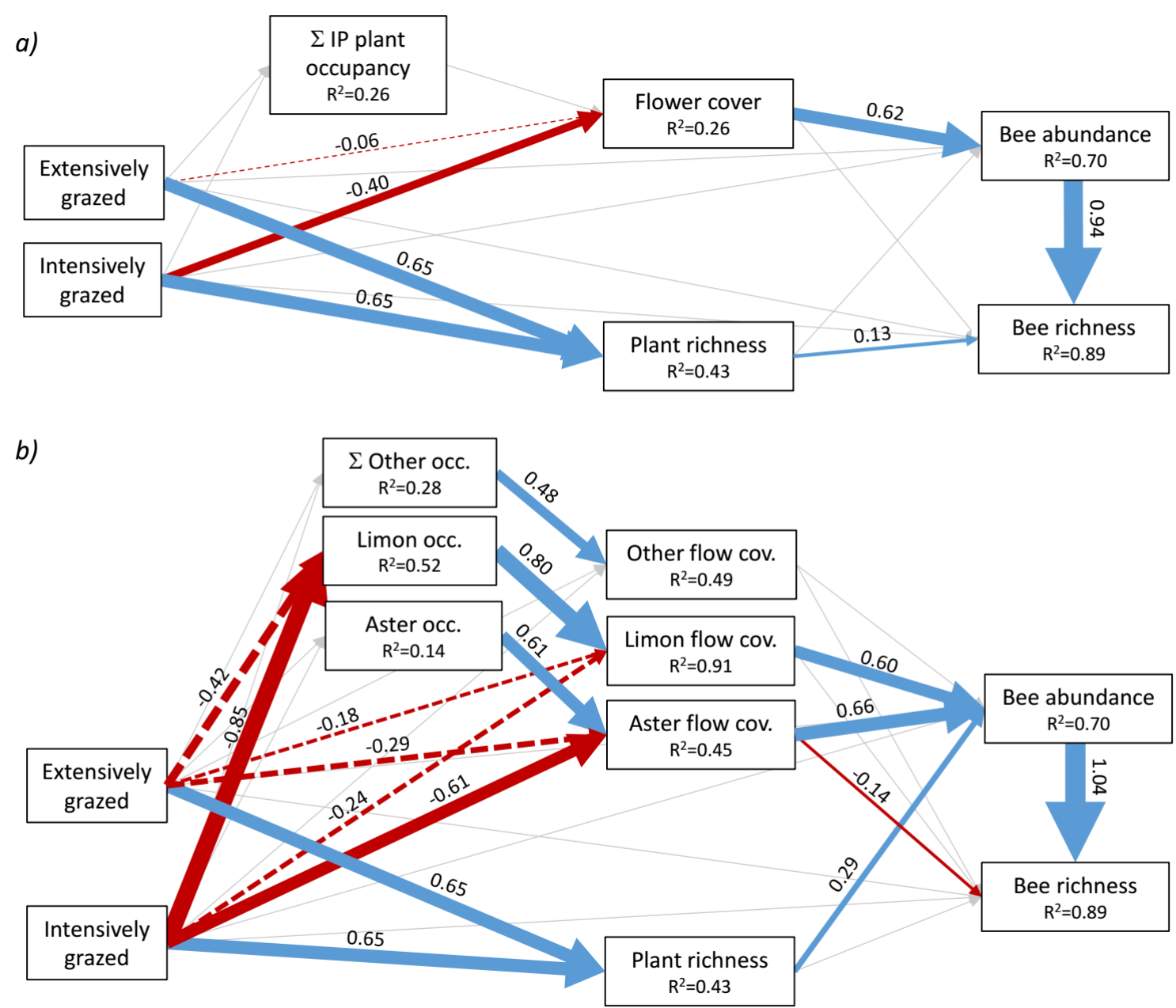

Fig. 4.4. The mechanisms of grazing effects investigated via a series of LMMs for $a$ ) all insectpollinated (IP) plant species combined and $b$ ) key plant species separated out. Significance was tested using likelihood ratio test (LRT): blue lines indicate significant positive effects, red lines indicate significant negative effects, grey lines indicate $P>0.05$. When grazing was significant by LRT, each grazing level was compared against absent grazing by $t$-test: a solid line represents $P<0.05$, a dashed line represents $P>0.05$. Line thickness for significant effects is weighted by standardised coefficient, which is also shown above the line (coefficients for grazing levels are relative to absent). Marginal $R^{2}$ values are for the full model, including the effect of time and weather variables, which have not been drawn: see Tables $\mathrm{C7}$ and C8 for full model results.

(PERMDISP). However, when $\beta$-diversity was partitioned into turnover and nestedness components, there were differences between grazing levels.

Grazing significantly affected nestedness-resultant dissimilarity (PERMANOVA $F=4.5$, $\mathrm{P}=0.042$; pairwise comparisons not significant) but had no effect on turnoverresultant dissimilarity (Table C9, Fig. C7), indicating that differences between bee communities at different grazing levels were driven by nestedness, rather than 
turnover. The effect of grazing on nestedness was not driven by differences in withingroup dispersion of nestedness (PERMDISP $F=1.7, \mathrm{P}=0.232$ ). Within-group dispersion due to turnover was lower in intensively grazed marshes than for other grazing levels ( $P$ ERMDISP F=10.1, $P<0.001$; pairwise comparisons $P<0.05$ ), indicating there was little species replacement across intensively-grazed marshes. These results did not change when only wild bees were considered.
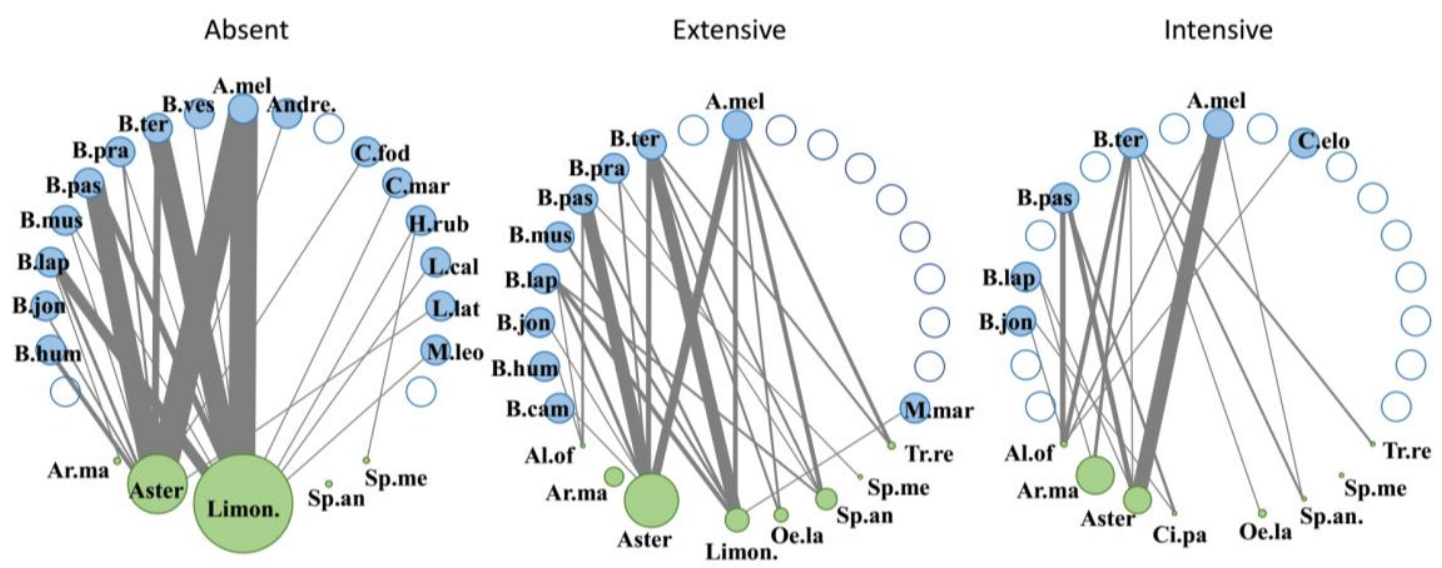

Fig. 4.5. Plant-pollinator network at each grazing level. Green circles are plant species (diameter proportional to flower cover), blue circles are bee species (weight of connector proportional to the square root of bee visits). See Tables B.1 and B.2 for species names.

\subsubsection{Comparing salt marshes with terrestrial habitats}

In July and August, the contribution of honeybees, bumblebees and solitary bees were $48 \%, 51 \%$ and $<1 \%$ in salt marshes, compared to $18 \%, 78 \%$ and $4 \%$ in terrestrial habitats. Habitat had a significant effect on the abundances of all bee types in JulyAugust (Table C10). When ranked with other habitats (Fig. 4.6), ungrazed salt marshes were the highest-ranked habitat for honeybee abundances, and the fifth highest-ranked habitat for bumblebees, but were less important for solitary bees. Grazed marshes were amongst the lowest-ranked habitats for wild bees, but extensively grazed marshes were the third highest-ranked habitat for honeybees. However, there was high variability within many habitat types, and habitat and weather predicted only a small proportion of the observed variation in bee abundance (marginal $\mathrm{R}^{2}=0.25,0.06,0.08$ for honeybee, bumblebee and solitary bee models). 

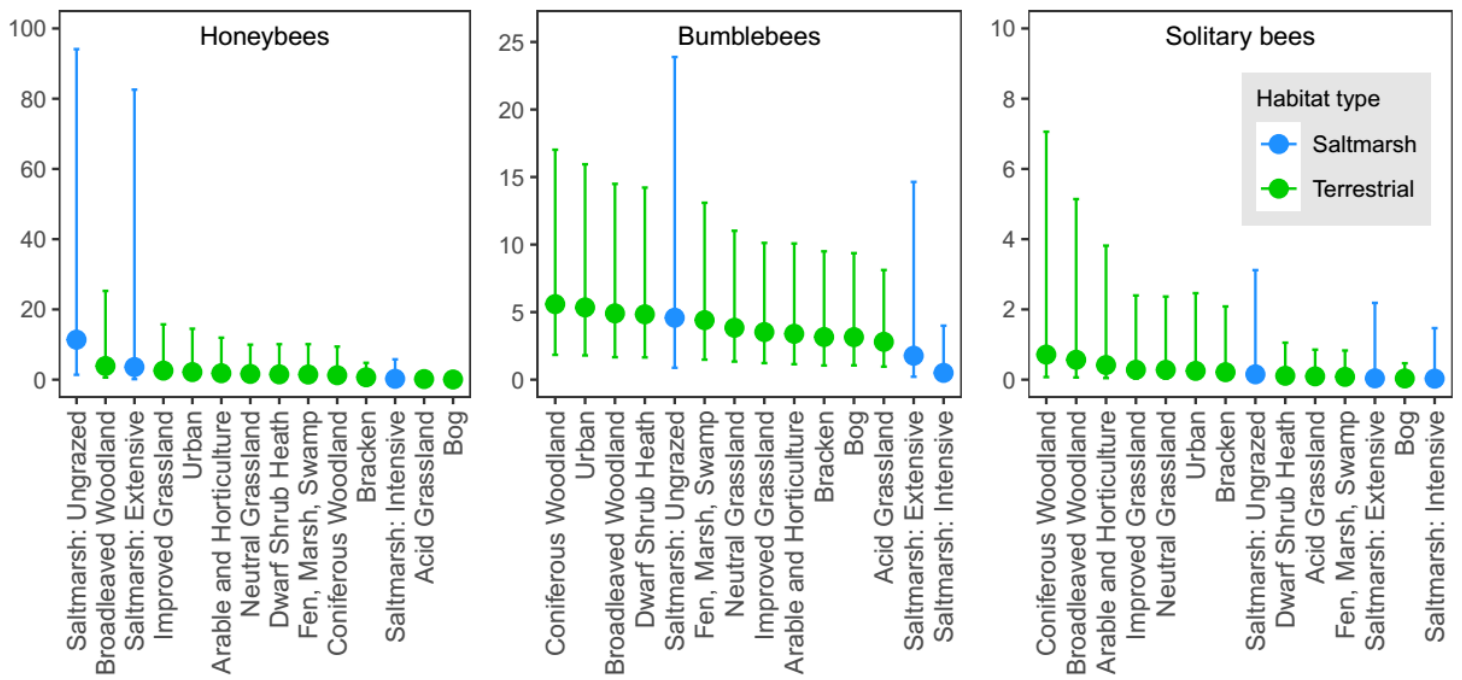

Fig. 4.6. Bee abundances in different habitats. Predicted abundances (per $200 \mathrm{~m}$ transect) and $95 \% \mathrm{Cls}$ of honeybees, bumblebees and solitary bees in July-August in different habitats.

\subsection{Discussion}

This study shows for the first time that salt marshes provide important foraging grounds for high numbers of honeybees and bumblebees, and that just two flowering plants account for practically all of this habitat provisioning service: Aster Tripolium pannonicum and Limonium spp. Livestock grazing reduces bee abundance and alpha diversity, and the effects are strongest with intensive grazing. There is a pattern of high species loss and low species replacement as grazing intensity increases.

Grazing impacts on bees principally operated via changes to the cover of two flowering plants (pathway 1, Fig. 4.1). Grazing reduced Limonium flower cover both directly and indirectly via reduced Limonium occupancy. Intensive grazing reduced Aster flower cover but did not affect Aster occupancy. Aster's higher grazing tolerance may be partly due to its occurrence in lower, wetter areas of the marsh (Adam 1981) which are less used by livestock (Sharps et al. 2017). Additionally, although Aster is highly palatable, Aster plants rarely die as a result of grazing (Nolte et al. 2013b), whereas Limonium is extremely susceptible to trampling and defoliation of young buds (Boorman 1967; Adnitt et al. 2007). 
Limonium and Aster were the two plants with the highest flower cover ( 30 and 33\% of total flower cover, respectively) but were disproportionately preferred by bees, accounting for $95 \%$ of total bee foraging visits. Both plants have high densities of flowers/florets, due to their dense clusters of small flowers (Limonium) and composite flowers (Aster), making them a good food source (Kirk and Howes 2012). Bees prefer to visit only one or two flower types to maximise foraging efficiency, and will target flowers that are abundant and have high food rewards (Free 1963; Gegear and Laverty 1998). Surprisingly, increasing Aster cover had a slight negative impact on bee richness. This is likely a result of controlling for bee abundance in our model: high Aster cover led to a large increase in bee abundance (predominantly honeybees), without a corresponding increase in bee richness.

Grazing had a positive effect on plant richness (pathway 2, Fig. 4.1), which in turn had a positive effect on bee abundance. However, combined 'other' flower cover had no effect on bee abundance, and only supported $5 \%$ of all observed bee visits for $37 \%$ of total flower cover. This may be because 'other' flower cover was dominated by Armeria maritima, which is rarely visited by bees on salt marshes (Eisikowitch and Woodell 1975), obscuring the effect of rarer flowering species. Our plant-pollinator networks indicate that these rarer species become more important in grazed marshes, where Aster and Limonium cover is reduced. Despite the benefits of increased plant richness, the positive effect of plant richness on bee abundance did not compensate for the negative effect of reduced flower cover. This result may be driven by the relatively limited flower and bee community of a salt marsh. We recommend similar investigation of the relative importance of flower richness and flower cover in other habitats to explore the universality of this process, as this could determine appropriate grazing management.

There was no evidence that grazed salt marshes harbour distinct assemblages of bees. Beta diversity between grazing levels was driven by nestedness rather than turnover. Two of the observed species (B. humilis, B. muscorum) are listed under the Environment (Wales) Act 2016 Section 7, which sets priority species for biodiversity conservation. Priority species were present on three ungrazed marshes, one extensively grazed, and no intensively grazed marshes. Due to the very low numbers 
of individuals observed for most species, we cannot make predictions about how individual species respond to grazing, but a general pattern of species loss with increased grazing is clear.

The number of bees foraging in salt marshes in July/August was not systematically higher or lower than numbers in terrestrial habitats, despite being surveyed in different years, covering a different range of latitudes and altitudes, and having slightly different survey methodology (only foraging bees recorded in salt marshes vs all bees in terrestrial habitats). However, the relative ranking of salt marshes varied depending on grazing management and bee guild. Compared to terrestrial habitats, ungrazed and extensively grazed marshes were well used by honeybees, with ungrazed marshes ranking as the top habitat. The presence of honeybees is strongly reliant on the presence of managed colonies in a location. Since coastal areas are more densely populated by humans than inland areas, this may partly explain the dominance of a domesticated bee species in saltmarsh habitats. Additionally, some beekeepers move hives around the landscape and may target salt marshes in late summer. Ungrazed marshes were in the top five habitats for bumblebees, but all saltmarsh categories were in the lower half of habitats for solitary bees. The scarcity of solitary bees on salt marshes may be due to their shorter foraging range (Greenleaf et al. 2007), limiting them to foraging areas close to their nest (which cannot be located on mid-low areas of a salt marsh due to tidal inundation).

The comparisons with terrestrial habitats were for July-August only, when Aster and Limonium are in bloom, but saltmarsh bee abundances were much lower in June. British bees are active from February to October (Falk and Lewington 2015) and need forage throughout this period. However, forage for honeybees and bumblebees more generally is low in mid-late summer (Couvillon et al. 2014; Timberlake et al. 2019), so the floral resources on salt marshes during this time may be particularly valuable to help fill this 'hunger gap'. The plant communities on British salt marshes are present across north-west Europe (Adam 1990), therefore the patterns from this study are likely to apply across temperate Europe. We have identified two consistently important plants for bees, meaning that European salt marshes can be rapidly assessed for their value to bees. American marshes also contain Limonium 
spp. and Asian marshes harbour Aster and Limonium spp. (Adam 1990), so the value of salt marshes for pollinators is likely to extend more widely.

\subsection{Conclusions and management implications}

Pollinator declines are primarily driven by habitat loss and agricultural intensification (Potts et al. 2010a). We have demonstrated that salt marshes can provide a vital resource for wild and managed bees during the 'hunger gap' of mid-late summer, contingent on appropriate management. Grazing, particularly intensive grazing, reduces the cover of key food plants, and the increase in plant diversity does not compensate for these reductions. Sensitively-managed salt marshes have the potential to contribute an important foraging habitat in a coastal landscape, enhancing the levels and reliability of pollination services (Garibaldi et al. 2011; Viana et al. 2012), and supporting wild species conservation.

This research provides evidence for three key management recommendations. Firstly, that to maximise pollinator presence salt marshes should manage for extensive or no grazing. We do not recommend abandonment of grazing, as this can lead to a homogenous grass-dominated habitat (Adam 1990). Therefore, grazed marshes should be maintained at - or reduced to - extensive levels, to encourage increased flower cover of preferred species. Secondly, that grazing management targeted towards high plant diversity does not necessarily benefit pollinators. Thirdly, that for both local and landscape diversity of bees, and conservation of priority species, long-term ungrazed marshes are optimal and intensively grazed marshes have little value.

\subsection{Acknowledgements}

Thanks to Kevin James, Josh Mutter, and Elizabeth Mills for their help with fieldwork, and to landowners who granted access to sites. Terrestrial survey data were provided by the Welsh Government's EU Exit \& Strategy Team, Department for Economy, Skills \& Natural Resources. 


\subsection{Supporting information}

The following supplementary material can be found in Appendix C.

Supplementary methods. Allocation of grazing levels

Supplementary methods. Calculating surrounding landscape

Supplementary methods. Terrestrial surveys for GMEP

Supplementary methods. Statistical packages

Table C1. Study site details

Table C2. Visual indicators used to allocate qualitative grazing levels

Table C3. Simplification of broad habitat types

Table C4. Bee abundance data for each marsh

Table C5. Insect-pollinated plant species on study salt marshes

Table C6. Net effect of grazing on bee community

Table C7. Mechanisms of grazing effect on bee community, all insect-pollinated plant species combined

Table C8. Mechanisms of grazing effect on bee community via key plant species

Table C9. $\beta$-diversity analyses

Table C10. Comparison with terrestrial habitats

Fig. C1. Allocation of grazing levels based on dung counts and qualitative assessment

Fig. C2. Network tested using LMMs with key plant species separated

Fig. C3. Bee species by abundance and prevalence

Fig. C4. Flower cover and bee visits, as a proportion of total

Fig. C5. Model predictions for plant responses to grazing

Fig. C6. Model predictions for bee responses to grazing and vegetation

Fig. C7. $\beta$-diversity analyses 


\section{Chapter 5: Mapping ecosystem services, trade-offs and multifunctionality in salt marshes: a case study in south Wales}

\subsection{Abstract}

Managers of multi-use ecosystems face conflicting demands from ecosystem service (ES) stakeholders and conservation objectives. To make decisions, managers require reliable information about the distribution of ES, factors affecting ES functioning, and potential trade-offs or synergies between ES. ES assessments often use land cover as a proxy for ES provision, but ES supply can vary widely within a single habitat. Here, I have used primary data to map the supply of seven ES for salt marshes in Carmarthen Bay, south Wales. I mapped a range of supporting/biodiversity, provisioning, regulating and cultural services for 67 marshes covering 3154 ha. I calculated ES multifunctionality for each marsh and tested whether marsh management, size, or location affects individual ES and multifunctionality measures. I explored potential trade-offs and synergies between ES using correlation analysis and ES bundles. I found that larger marshes had the highest levels of provisioning and cultural services, and the highest multifunctionality values. Salt marsh managed as common land for multiple graziers had the highest values for grazing and winter bird habitat, and the highest average ES functioning values. Carbon was highest in private/tenant farmed marshes and marshes further upriver, whereas bee habitat was highest in marshes managed by conservation bodies/local councils and marshes closer to the river mouth. Only $4 \%$ of the total saltmarsh area achieves moderate levels of bee habitat. Bee habitat trades-off against grazing, due to a direct influence of livestock on bee habitat quality. Birdwatching is precluded in many marshes, due 
to lack of public access. I recommend that cultural services can be increased across the landscape by increasing access and facilities for birdwatchers, and that biodiversity/supporting services should be increased by incentivising reduced grazing levels in some marshes to increase the cover of quality bee habitat. These results can feed into ES assessments across the wider landscape, and demonstrate that salt marshes cannot be treated as a homogeneous habitat, but must be differentiated by management, size and location.

\subsection{Introduction}

A major challenge facing managers of multi-use ecosystems is how to balance competing demands for ecosystem services (ES) and conservation objectives. Often, maximising the provision of one ES can drive declines in others (e.g. Allen 2015, Kim et al. 2016, Turner et al. 2014). In particular, increasing the supply of provisioning services, such as agricultural production, commonly leads to declines in regulating, cultural and supporting services (MA 2005a; Allan et al. 2015; Cavender-bares et al. 2015; Holt et al. 2016). Provisioning services often have clearly defined economic benefits (e.g. livestock production) so are commonly favoured by landowners over ES with less tangible benefits (MA 2005a; Rodríguez et al. 2006; Nilsson et al. 2017). To sensitively manage a landscape supplying multiple supporting, provisioning, regulating and cultural services, it is necessary to understand what ES perform well or poorly, the factors that drive good or poor supply of multiple ES, and identify any interactions (trade-offs and synergies) among ES.

ES maps are an important and widely applied tool, used to display and evaluate ES provision across a landscape, and to support policy and environmental resource management (Maes et al. 2012). ES maps can efficiently communicate complex spatial information and identify patterns in ES supply and demand across a range of spatial scales (Burkhard and Maes 2017). Mapping of ES bundles (coherent sets of ES that repeatedly appear together across space or time, Raudsepp-Hearne et al. 2010) can help predict synergies and trade-offs between ES (Spake et al. 2017). However, spatial analysis does also have some disadvantages. ES maps can be used to detect pattern-based multifunctionality and ES associations, but do not resolve the 
processes underpinning these patterns (Mastrangelo et al. 2014): spatial interaction between ES may result from one ES directly impacting on others, or from multiple ES reacting to a common driver (Bennett et al. 2009). Maps of ES represent only a snapshot in time, but ES supply can change within and across years (Renard et al. 2015), and there may be lags in time between the changing of a driver and the supply of an ES (Spake et al. 2017). Often, spatial analysis is hindered by lack of data about ES provision. Scientists commonly use land use/land cover (LULC) as a proxy to model ES provision, where each type of LULC is allocated an estimated ES value (Bennett et al. 2009; Broekx et al. 2013). However, the ES supplied by a particular habitat can vary widely depending on habitat quality and management (Gos et al. 2016; Soliveres et al. 2016). For example, salt marshes may be grazed or not grazed by livestock, with significant consequences for the ecosystem properties of the marsh and therefore for ES provision (Chapter 2: Davidson et al. 2017). To gain a more accurate picture of ES supply, drivers and interactions, it is therefore necessary to examine how ES provision changes within a LULC category using primary data (Bennett et al. 2009; Spake et al. 2017). These types of ES relationships (within a LULC class) remain much less studied than ES relationships across LULC types (Spake et al. 2017).

Here, I have investigated the provision of, and relationships among, ES in salt marshes, using a case study system in south Wales, UK. UK salt marshes supply a variety of ES to multiple stakeholders, from a local to a global scale. These ES include the provision of grazing land for local farmers, recreational space for local residents and visitors, the capture and long-term storage of atmospheric carbon, and the provision of diverse habitat to support wildlife such as insects and birds (Jones et al. 2011). The high variety and quality of benefits accruing from salt marshes has led to increasing recognition of their value to society (Costanza et al. 1997; Woodward and Wui 2001; Barbier et al. 2011). However, because these multiple ES can trade-off against each other (e.g. van Loon-Steensma \& Vellinga 2013; Sharps et al. 2015), governance bodies are left with conflicting management requirements, making it difficult to set priorities and desired outcomes for a site. To the best of my knowledge, multiple ES have not been previously been mapped in salt marshes. 
I have gathered, analysed and mapped data from a variety of academic, public, charitable and private bodies to evaluate seven ES supplied by salt marshes in Carmarthen Bay in south Wales. The study site, which covers two estuaries containing over a third of the total area of salt marsh in Wales, was selected because it is representative of salt marsh vegetation across temperature Europe (Adam 1990), because a wide variety of ecological data have been collected across the site, and because there are many potential conflicts due to the multiple sensitive ecological features present and widespread agriculture in the area. I mapped the seven individual ES as well as multiple measures of ES multifunctionality (based on averaging, diversity, and threshold approaches) to assess the spatial distribution of ES and determine what variables (marsh management, size and location) drive high ES provision and multifunctionality. I also investigated potential trade-offs and synergies between ES using correlation analysis, and by identifying and mapping ES bundles using cluster analysis.

\subsection{Methods}

\subsubsection{Case study location}

Carmarthen Bay in South Wales comprises two estuaries - the Three Rivers Estuary and the Loughor Estuary - and holds 3154 ha salt marsh, making up 34.5\% of the total saltmarsh area in Wales (Fig. 5.1). The bay and estuaries are designated as a Special Area of Conservation (SAC) as they contain multiple Annex I habitats protected under the 1992 EC Habitats Directive (European Council Directive 92/43/EEC). The site also contains two Special Protected Areas (areas protected for rare and vulnerable birds under European Council Directive 2009/147/EC), a Ramsar Wetland of International Importance, and multiple Nature Reserves and Sites of Special Scientific Interest. Natural Resources Wales (NRW) have overall responsibility for monitoring the site and maintaining it in a favourable conservation status. However, NRW have identified several evidence gaps relating to current grazing management, required management relating to the conservation interest in different parts of the site, and the distribution of ES provided by the salt marsh (Davidson and Griffin 2018). By collating and analysing data from various sources I aim to highlight 
areas of high and low ES provision, identify the key factors affecting ES multifunctionality, and recognise potential ES trade-offs. This will allow the most important marshes to be recognised, and help target management intervention toward marshes that can be improved.

I assessed ES provision at the scale of individual salt marshes. Each salt marsh was defined and named as described in the NRW 2012 Carmarthen Bay and Estuaries condition monitoring report (Pauls 2017) so as to be relevant for the management body. Where a salt marsh fell under multiple ownership, I further divided it into subsections. Salt marshes and salt marsh subsections are hereafter referred to as 'marshes'. I assessed ES provision for 67 marshes in total, although data were not available for all ES for every marsh. Because marshes vary widely in size (0.1 - 573.5 ha) I normalised ES for area, and tested whether marsh area had a significant effect on individual ES supply and ES multifunctionality.

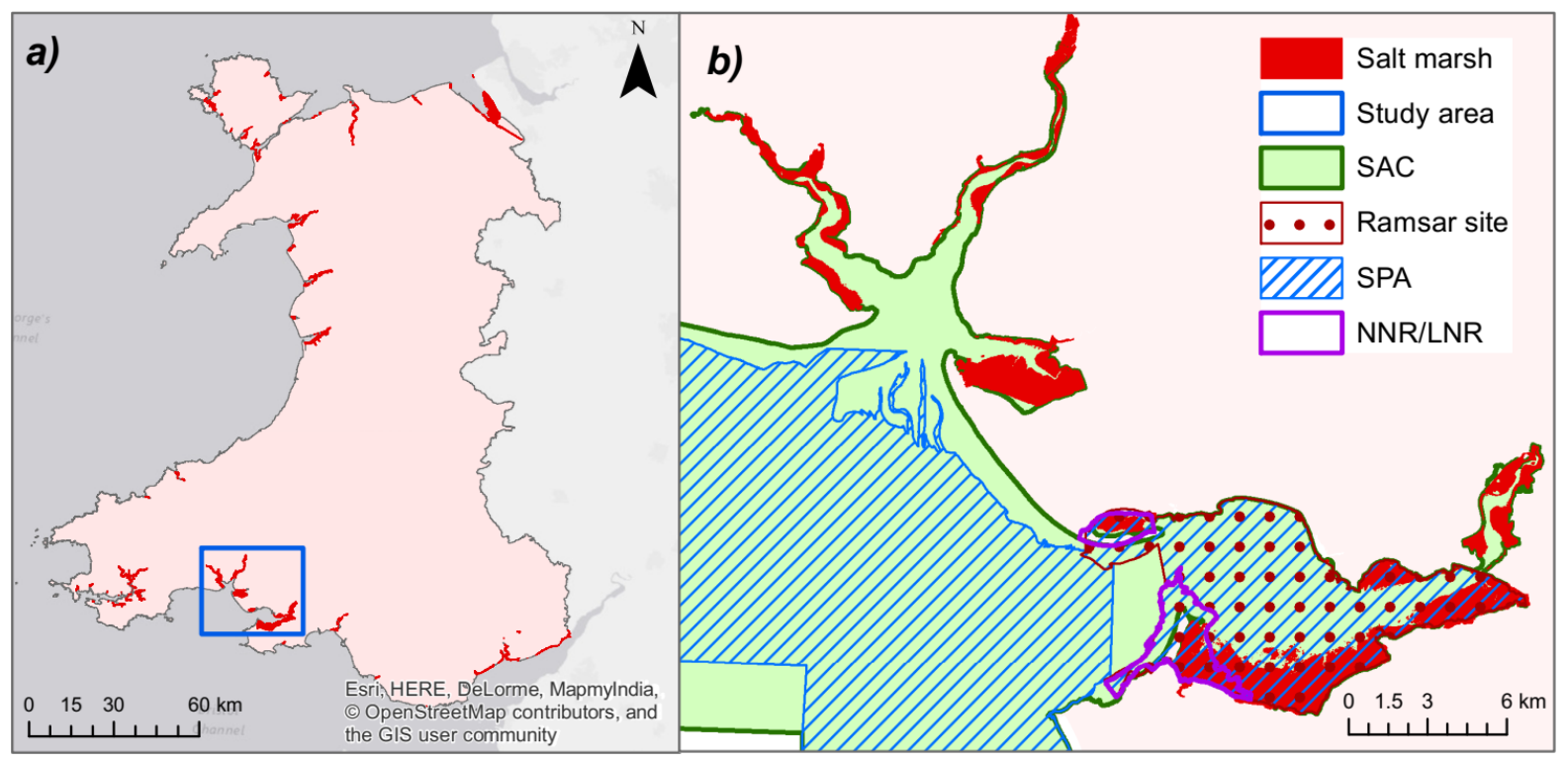

Fig. 5.1. a) Location of case-study site (Carmarthen Bay) in South Wales, UK, and b) protected designations at study site (not including Sites of Special Scientific Interest). Carmarthen Bay contains the Three Rivers Estuary to the northwest and the Loughor Estuary to the southeast. SAC = Special Area of Conservation, SPA = Special Protected Area, NNR/LNR = National/Local Nature Reserve. 


\subsubsection{Ecosystem service selection and quantification}

I assessed seven ES across the study area, including biodiversity/supporting services $(n=3)$, provisioning $(n=1)$, regulating $(n=1)$ and cultural $(n=2)$ services. ES were selected based on their importance at a local or global level, and data availability for the study area. Measurable ES proxies were selected as an indicator of supply, and the ES value for each marsh was calculated using published and unpublished data sourced from various private, public, charitable and academic institutions. All ES proxies were rescaled as a percentage of maximum functioning $(0-100 \%)$ to allow meaningful comparisons between ES measured on different scales (Table 5.1). I also allocated a confidence level of low, medium and high for each ES value. Methods used to calculate each ES proxy and confidence levels are described in full in Appendix $D$, and each ES is described in brief below.

\section{Biodiversity/supporting services}

Supporting services (variously termed intermediate services, ecosystem processes, or habitat services under different frameworks) are those that underpin the production of all other services. Here I assessed three biodiversity measures: plant diversity, the provision of overwintering habitat for saltmarsh-specialist birds and the provision of foraging habitat for bees. Biodiversity measures were selected because high biodiversity directly supports other services (e.g. bird diversity supports birdwatching, pollinator diversity supports crop pollination), maintenance of high diversity provides an 'option' value for the future (Gascon et al. 2015), and the study area contains multiple protected areas, conferring a management duty to preserve biodiversity. 'Plant diversity' was selected because high plant diversity can support high multifunctionality in grasslands (Allan et al. 2015; Meyer et al. 2016). There are many definitions of diversity (e.g. diversity within sites, diversity between sites, genetic diversity); here I mapped the simplest measure - plant species richness per transect, which was obtained from extensive vegetation surveys conducted by NRW under the Water Framework Directive (WFD) in 2013 and 2015. 'Bird habitat' was selected because salt marshes are an important feeding ground for many species of overwintering birds and also provide high tide roosts (Adnitt et al. 2007). The Special 
Protected Area designations within the study site relate to the overwintering bird populations, therefore this service is very relevant to site management considerations. Here I mapped the number of saltmarsh-specialist overwintering birds that regularly occur on each marsh, weighted by the conservation status (Red, Amber, Green) of each species. Data were obtained from monthly surveys conducted for the British Trust for Ornithology's Wetland Bird Survey (WeBS) from 2007/2008 2016/2017. 'Bee habitat' was mapped because salt marshes can provide high-value bee-foraging habitat (Chapter 4) and under the Action Plan for Pollinators in Wales (Welsh Government 2013a), Wales has a duty to provide diverse and connected flower rich habitats to support pollinators. I used the estimated grazing level in each marsh (see below), and plant community data from WFD surveys to predict the average number of bees per $1 \mathrm{~km}$ transect in each marsh.

\section{Provisioning services}

Provisioning services provide material goods. This is where overexploitation can occur and where there is a risk of strong trade-offs with other services: high values of provisioning services can have detrimental impacts on overall habitat health and functioning (MA 2005a; Cavender-bares et al. 2015; Holt et al. 2016). 'Livestock grazing' is one of the most common uses of saltmarsh land (Adam 1990). It is important to understand the distribution of livestock because grazing can affect other ES (Chapter 2) and can be relatively easily influenced and controlled by management. I estimated grazing levels as ungrazed, lightly, moderately or heavily grazed based on observations from Carmarthen Bay condition monitoring in 2012, sward height and descriptions from 2013/2015 WFD surveys, and self-reported grazing levels from owner/occupier interviews conducted in 2017 (Appendix E).

\section{Regulating services}

Regulating services provide benefits by regulating ecosystem processes such as climate regulation, natural hazard regulation or waste management. I mapped 'Carbon storage' which contributes to climate regulation. Salt marshes store most carbon below ground, and can be an extremely effective carbon sink because the 
anaerobic conditions slow decomposition of organic material, high sulphates inhibit methane-producing bacteria, and continual accretion allows ongoing accumulation of carbon without reaching equilibrium (Chmura 2009). I used existing survey data from Welsh saltmarsh soils (Skov et al. 2016, D. de Battisti unpublished data) to estimate the carbon stored in the top $10 \mathrm{~cm}$ of each marsh (tonnes $\mathrm{Cha}^{-1}$ ) based on vegetation community and soil type.

\section{Cultural services}

Cultural services are non-material benefits obtained from ecosystems. Because they can be difficult to quantify, they are often left out of ES assessments (Chan et al. 2011). Cultural services can have negative effects on a habitat due to, for example, human disturbance of wildlife, trampling of plants and soils, pollution, or construction of infrastructure. Therefore, while high levels of cultural services are generally positive, care must be taken that they do not inadvertently degrade the habitat and wildlife they are there to enjoy (Stigner et al. 2016). 'Birdwatching' has been mapped because salt marshes attract high numbers of diverse birds, and are therefore a favoured spot for birdwatchers (Adnitt et al. 2007; Green and Elmberg 2014). I mapped the potential value for winter birdwatching based on winter bird diversity and abundance, and other valued features of the marsh, such as accessibility. This does not necessarily reflect the realised ES value in terms of number of visits, but does reflect the traits that are most desired by birdwatchers, as assessed by an expert opinion survey (Appendix F). I mapped 'Wildfowling' because of the availability of comprehensive data for the entire site, and because of the potential to conflict with conservation priorities. I have classed wildfowling as a recreational pursuit, under cultural services, but it could also be considered a provisioning service for providing wild food.

Salt marshes provide many other important services not represented here (e.g. coastal protection, supporting coastal fisheries, water quality regulation, breeding bird habitat) but it was not possible to map these due to limitations in data availability or the complexity of modelling. 
Table 5.1. Summary of ecosystem services mapped for the project and upper and lower boundaries used to rescale values. Full details of methods used to assign values for each ES are given in Appendix D.

\begin{tabular}{|c|c|c|c|c|}
\hline $\begin{array}{l}\text { ES } \\
\text { category }\end{array}$ & Service & Proxy & $0 \%$ boundary & $100 \%$ boundary \\
\hline \multirow{3}{*}{ 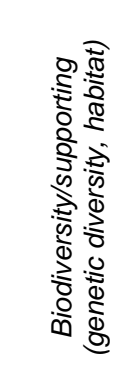 } & $\begin{array}{l}\text { Plant } \\
\text { diversity }\end{array}$ & $\begin{array}{l}\text { Average number of plant species per } \\
\text { transect }\end{array}$ & $\begin{array}{l}\text { Minimum number of } \\
\text { species recorded on } \\
\text { a single transect in } \\
\text { the study area (10) }\end{array}$ & $\begin{array}{l}\text { Maximum score } \\
\text { recorded in study } \\
\text { area (34.5) }\end{array}$ \\
\hline & Bird habitat & $\begin{array}{l}\text { Number of saltmarsh-specialist } \\
\text { overwintering bird species regularly } \\
\text { recorded in the area, weighted by } \\
\text { conservation status }\end{array}$ & 0 species & $\begin{array}{l}\text { Maximum score } \\
\text { recorded in CBE } \\
(29)\end{array}$ \\
\hline & Bee habitat & $\begin{array}{l}\text { Predicted average number of bees } \\
\text { foraging on a } 1 \mathrm{~km} \text { transect, based on } \\
\text { plant composition and grazing levels }\end{array}$ & $\begin{array}{l}\text { Minimum prediction } \\
\text { for study area }(0)\end{array}$ & $\begin{array}{l}\text { Maximum } \\
\text { prediction for } \\
\text { study area (40.7) }\end{array}$ \\
\hline 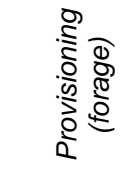 & $\begin{array}{l}\text { Livestock } \\
\text { grazing }\end{array}$ & $\begin{array}{l}\text { Estimate of ungrazed, lightly, } \\
\text { moderately or heavily grazed }\end{array}$ & Ungrazed & Heavy grazing \\
\hline 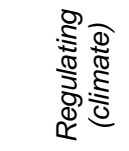 & $\begin{array}{l}\text { Carbon } \\
\text { storage }\end{array}$ & $\begin{array}{l}\text { Tonnes of organic carbon per hectare } \\
\text { (top } 10 \mathrm{~cm} \text { of soil) }\end{array}$ & $\begin{array}{l}\text { Minimum value } \\
\text { recorded in the } \\
\text { study area (22) }\end{array}$ & $\begin{array}{l}\text { Maximum value } \\
\text { calculated for } \\
\text { study area (53.7) }\end{array}$ \\
\hline \multirow{2}{*}{ 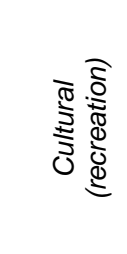 } & Birdwatching & $\begin{array}{l}\text { Combined score based on public } \\
\text { access, bird diversity, bird } \\
\text { abundance, accessibility, availability } \\
\text { of bird hide, tranquillity }\end{array}$ & $\begin{array}{l}\text { Score of } 0 \text { (no } \\
\text { public access) }\end{array}$ & $\begin{array}{l}\text { Maximum } \\
\text { potential score } \\
(100)\end{array}$ \\
\hline & Wildfowling & $\begin{array}{l}\text { Number of birds shot per } \mathrm{km} \text { of } \\
\text { foreshore }\end{array}$ & $\begin{array}{l}\text { Minimum value } \\
\text { recorded in the } \\
\text { study area }(0)\end{array}$ & $\begin{array}{l}\text { Maximum value } \\
\text { recorded in study } \\
\text { area (47) }\end{array}$ \\
\hline
\end{tabular}

\subsubsection{Multifunctionality and interactions among services}

\section{Calculating multifunctionality}

ES multifunctionality is the capacity of a landscape or ecosystem to simultaneously provide multiple ES (Manning et al. 2018). There is no universally agreed measure of ES multifunctionality, so I used three approaches to assess the level of multifunctionality for each marsh. I (i) calculated the average function level across the seven ES; (ii) calculated ES diversity as the Simpson's diversity index to give a measure of ES evenness across marshes (Raudsepp-Hearne et al. 2010); and (iii) calculated the number of ES exceeding multiple supply thresholds (Byrnes et al. 2014) which I defined as $\geq 30 \%$ (at least a low supply), $\geq 50 \%$ (at least a moderate supply) and $\geq 70 \%$ (a high supply). 
I used linear regression analysis to test whether individual ES and the various measures of ES multifunctionality were significantly affected by marsh management (common land with multiple graziers, private owner or tenant farmer, under conservation body or local council management), marsh size (area in ha), or marsh location (distance from river mouth).

\section{Investigating interactions among ecosystem services}

To identify potential trade-offs and synergies between ES, I calculated the Pearson's correlation coefficient between pairs of ES. I also assessed whether individual ES significantly correlated with the multifunctionality measures, and whether multifunctionality measures correlated with each other. I further investigated spatial relationships among ES by identifying groups of marshes with similar sets of ES (ES bundles) using K-means cluster analysis (Raudsepp-Hearne et al. 2010). The optimum number of bundles were estimated using the fpc package (Henning 2019), which calculates the optimum average silhouette width (i.e. distance between clusters). Principal Component Analysis was used to analyse the variation in the seven ES, and help identify the dominant ES characteristics of each bundle (Raudsepp-Hearne et al. 2010). ES bundles were mapped to assess the spatial distribution of bundles, and the total area covered by each bundle was calculated, to determine whether any bundles were strongly over- or under-represented within the study area.

I only assessed multifunctionality and ES bundles for marshes that had valuations for all seven ES. However, 38 of the 67 study marshes, covering 505 ha (16\% of the total salt marsh area), did not fall within a Wetland Bird Survey (WeBS) sector. To increase the sample size, I assumed that all marshes that were not within a WeBS sector had low value for wetland bird abundance and diversity, as WeBS surveys prioritise areas that are well-used by waterbirds (British Trust for Ornithology 2019). Therefore, all marshes that fell outside WeBS sectors were allocated a value of $20 \%$ for bird habitat value (to fall under the 'low' supply threshold, the lowest recorded value within a WeBS sector was 31\%), and were allocated scores equal to the lowest recorded values for abundance and diversity when calculating birdwatching value. This gave a sample size of 51 marshes covering $98 \%$ of the total salt marsh area for the 
multifunctionality/ES bundle analysis. I did not use these estimated values when testing individual ES (effect of management, size and location on individual ES; correlations between ES-ES and ES-multifunctionality). Each marsh was allocated an overall confidence level for the calculated multifunctionality values and ES bundle by averaging across the confidence values for each individual ES, where $0=$ =assumed value, $1=$ low, $2=$ =medium, 3=high confidence. All graphical analyses were conducted in ArcGIS Desktop 10.5.1 and all statistical analyses were conducted in R Studio running R3.5.2 (R Core Team 2018).

\subsection{Results and Discussion}

I assessed seven ES for 67 marshes within Carmarthen Bay and Estuaries. However, data were not available for all marshes, particularly the smallest marshes, and the number of services assessed per marsh ranged from 1-7 (mean=5.2, mode=7). I was able to assess all seven ES for 26 marshes, covering 2620 ha ( $83 \%$ of the total salt marsh area). The majority of marshes were managed by a private owner or tenant (27), with far fewer being run by conservation bodies/local councils (6) or grazed by multiple graziers as common land (5; management details not obtained for 29 of the smallest marshes). However, marsh management covaries with marsh size (ANOVA, $\mathrm{df}=2,35, \mathrm{~F}=4.28, \mathrm{P}=0.02$ ): common land is significantly larger than marshes managed by private or tenant farmers (Tukey's HSD, $\mathrm{t}=2.92, \mathrm{P}=0.02$ ), with marshes managed by conservation bodies/local councils being of intermediate size. The average (range) marsh size is 47.1 ha $(0.1-573.5)$ and distance to the river mouth is $7.8 \mathrm{~km}(1.1-$ 14.7). Marshes upriver are generally smaller than those close to the river mouth, but the correlation between area and distance was not significant $(r=-0.13, P=0.29)$.

\subsubsection{Individual ecosystem services}

\section{Biodiversity/Supporting services}

The raw and rescaled ES values for each marsh are presented in Appendix D (Tables D4-D5), along with heat maps and confidence levels (Figs D1-D2). Plant diversity ranged from 14 to 34.5 species per transect, and marshes closer to the river mouth 
had significantly higher diversity than those upriver (Table 5.2). In the decade for which bird habitat value was assessed (2007/8 - 2016/17), there were 22 species of overwintering saltmarsh-specialist birds recorded across the study area, with an average of 13 species per WeBS sector. The two highest performing sectors each had 2 regularly occurring red species, 15 regularly occurring amber species, and 2 regularly occurring green species. These two high-value sectors were allocated confidence levels of low and medium, as they were surveyed in just 3 and 5 years during the survey decade, respectively. Nevertheless, the results indicate these sectors provide vital habitat for overwintering saltmarsh birds. The survey sector with the lowest value was regularly visited by five overwintering saltmarsh species (1 red, 2 amber, 2 green), highlighting how even 'low value' salt marshes are important for overwintering birds. There was no clear pattern to the distribution of low- and high-ranking marshes for bird habitat.

Predicted bee abundance ranged from 0 to 40.7 bees per transect km, but only 2 of the marshes achieved a high value ( $\geq 70 \%$ of maximum), and just 9 of the 51 assessed marshes achieved at least a low value $(\geq 30 \%)$ for bee habitat provision. These results clearly indicate that the majority of salt marshes within the study area are strongly under-performing in terms of their potential as a bee foraging habitat. Bee habitat value decreased with increasing distance from the river mouth, which is likely related to the decreasing plant diversity and increasing grazing intensity further from the river mouth (Table 5.2). Bee habitat values were highest in conservation body/council managed marshes, and lowest in common land, which again is likely due to the grazing levels within these respective marshes (Table 5.2)

\section{Provisioning services}

Across the study site, the majority of marsh area was classified as heavily ( $29 \%$ of total area, 911 ha) or moderately grazed (50\%, 1581 ha). Only $11 \%$ (225 ha) was ungrazed, and the remaining 10\% (306 ha) was lightly grazed. Salt marshes are valuable as grazing land, because they are self-fertilizing and highly-productive (nutrients brought in on incoming tides), and low in pathogens (organic material washed away on ebbing tides; Lambert 2000). Owner/occupier interviews revealed 
that saltmarsh grazing can be very important to overall farm income (Appendix E). The marshes of the north Gower have been grazed by commoners for many generations, and the saltmarsh lamb reared there are sold as a prized seasonal delicacy (Prince 2011; Gower Salt Marsh Lamb 2019). The grazing across Carmarthen Bay therefore has both heritage and economic value. Grazing levels were heaviest on common land and lightest on land managed by conservation bodies/councils (Table 5.2). Grazing intensity increased with increasing marsh area, perhaps due to increased accessibility and practicality of grazing large marsh areas (or simply because common grazing marshes tend to be very large and also heavily grazed). Grazing intensity also increased with increasing distance from the river mouth.

\section{Regulating services}

The total organic carbon stock for Carmarthen Bay salt marshes was estimated at 125,165 tonnes $C$ in the upper $10 \mathrm{~cm}$. Carbon stocks increased with increasing distance from the river mouth (Table 5.2), as the vegetation community changes from high coverage of Spartina anglica (which has high salinity tolerance and lower associated carbon stocks) to marshes with high coverage of Juncus maritimus and $J$. gerardii (lower salinity tolerance, higher associated carbon stocks). Carbon stocks were highest in privately owned marshes and lowest in those managed by conservation bodies/councils, which is likely because privately owned marshes tend to be located further upriver. Similar patterns of increasing carbon storage with increased freshwater influence are seen in Australian and US tidal marshes (Loomis and Craft 2010; Macreadie et al. 2017).

\section{Cultural services}

The top four marshes for birdwatching value were located along a large, contiguous expanse of salt marsh on the southern shore of the Loughor Estuary. These marshes all had high bird diversity (at least 9 of a potential 22 species of saltmarsh-specialist overwintering birds). Three benefitted from very high accessibility, as there is a road and carparking immediately adjacent to the marshes, while the fourth benefitted from a bird hide. Where birdwatching value was low, this was generally due to a lack 
of public access onto the land. Larger marshes had higher birdwatching value, as did common land and conservation body/council-managed marshes, which is likely due to the higher accessibility of these marshes and presence of hides. There are three licenced wildfowling clubs operating across five shooting grounds (covering 21 marshes) in Carmarthen Bay. Shooting is prohibited outside these areas. Mallard, Teal and Wigeon were the most commonly shot birds, and each active wildfowler averaged 5.0 birds per season, although this varied from ground-to-ground (ranging from 2.7 -9.9). One club in particular was responsible for the majority of birds shot (averaging 47 birds $\mathrm{km}^{-1}$ foreshore $\mathrm{yr}^{-1}$ ), with the next highest value shooting ground achieving less than half of this value. Wildfowling value increased with increasing marsh size (Table 5.2).

Table 5.2. Results of linear regression analysis testing the effect of marsh management, area and location on individual ecosystem service (ES) and multifunctionality measures (MF). Where management was found to be significant, we used Tukey's HSD to test for significant differences between each management type ( $\mathrm{C}=$ common land with multiple graziers, $\mathrm{PT}=$ private owner or tenant farmer, $\mathrm{CC}=$ conservation body or council). < indicates significantly less than, $\leq$ indicates not significantly less than. Pla=plant diversity, Bir=bird habitat, Bee=bee habitat, Gra=grazing, Car=carbon storage, Wat=birdwatching, Wil=wildfowling, $\beta=$ standardised regression coefficient. Significant effects $(P \leq 0.05)$ are highlighted in bold. $\beta$ shows standardised $\beta$ coefficients.

\begin{tabular}{|c|c|c|c|c|c|c|c|c|c|c|c|c|c|c|c|}
\hline & \multicolumn{5}{|c|}{ Management } & \multicolumn{5}{|c|}{ Area (ha, log transformed) } & \multicolumn{5}{|c|}{ Distance from river mouth $(\mathrm{m})$} \\
\hline & $\mathrm{df}$ & $\mathrm{F}$ & $P$ & $\mathrm{R}^{2}$ & Contrasts & df & $\mathrm{F}$ & $P$ & $\beta$ & $\mathrm{R}^{2}$ & df & $\mathrm{F}$ & $P$ & $\beta$ & $\mathrm{R}^{2}$ \\
\hline \multicolumn{16}{|l|}{ ES } \\
\hline $\mathrm{Pla}$ & 2,31 & 0.91 & 0.413 & .06 & & 1,49 & 0.00 & 0.950 & -0.01 & .00 & 1,49 & 5.33 & 0.025 & -0.31 & .10 \\
\hline Bir & 2,25 & 0.11 & 0.897 & .01 & & 1,27 & 0.20 & 0.661 & -0.09 & .01 & 1,27 & 0.03 & 0.870 & -0.03 & .00 \\
\hline Bee & 2,31 & 4.82 & 0.015 & .24 & $\mathrm{C} \leq \mathrm{PT}<\mathrm{CC}$ & 1,49 & 0.09 & 0.766 & 0.04 & .00 & 1,49 & 9.49 & 0.003 & -0.40 & .16 \\
\hline Gra & 2,35 & 9.62 & $<.001$ & .35 & $\mathrm{CC}<\mathrm{PT}<\mathrm{C}$ & 1,53 & 10.7 & 0.002 & 0.41 & .17 & 1,53 & 5.84 & 0.019 & 0.32 & .10 \\
\hline Car & 2,35 & 3.99 & 0.028 & .19 & $\mathrm{CC} \leq \mathrm{C} \leq \mathrm{PT}$ & 1,48 & 2.52 & 0.119 & 0.22 & .05 & 1,48 & 5.42 & 0.024 & 0.31 & .10 \\
\hline Wat & 2,27 & 9.41 & $<.001$ & .41 & $\mathrm{PT}<\mathrm{CC} \leq \mathrm{C}$ & 1,41 & 23.1 & $<.0001$ & 0.60 & .36 & 1,41 & 1.06 & 0.310 & -0.16 & .03 \\
\hline Wil & 2,35 & 0.61 & 0.550 & .03 & & 1,65 & 11.8 & 0.001 & 0.39 & .15 & 1,65 & 2.09 & 0.153 & 0.18 & .03 \\
\hline \multicolumn{16}{|l|}{$M F$} \\
\hline Average & 2,31 & 4.05 & 0.027 & .21 & $\mathrm{CC}<\mathrm{PT} \leq \mathrm{C}$ & 1,49 & 47.5 & $<.0001$ & 0.70 & .49 & 1,49 & 0.64 & 0.429 & 0.11 & .01 \\
\hline Diversity & 2,31 & 0.20 & 0.820 & .01 & & 1,49 & 14.5 & $<.001$ & 0.48 & .23 & 1,49 & 0.04 & 0.850 & -0.03 & .00 \\
\hline$E S \geq 30 \%$ & 2,31 & 0.41 & 0.669 & .03 & & 1,49 & 24.7 & $<.0001$ & 0.58 & .34 & 1,49 & 1.57 & 0.216 & 0.18 & .03 \\
\hline$E S \geq 50 \%$ & 2,31 & 2.92 & 0.069 & .16 & & 1,49 & 18.6 & $<.0001$ & 0.53 & .28 & 1,49 & 1.46 & 0.233 & 0.17 & .03 \\
\hline$E S \geq 70 \%$ & 2,31 & 2.48 & 0.100 & .14 & & 1,49 & 24.5 & $<.0001$ & 0.58 & .33 & 1,49 & 0.03 & 0.868 & -0.02 & .00 \\
\hline
\end{tabular}




\subsubsection{Multifunctionality and interactions among services}

\section{Patterns in ecosystem service multifunctionality}

The use of multiple measures of multifunctionality (averaging, diversity and threshold approaches; Figs 5.2-5.6) helps to clarify different concepts of multifunctionality, and identify how they vary from marsh-to-marsh, as well as highlighting common patterns. One marsh in particular (highlighted with an arrow in Figs 5.2-5.5) shows as a multifunctionality hotspot for all multifunctionality measures except 'no. services $\geq 70 \%$ '. This large marsh is moderately grazed by a tenant farmer, supplies high levels of carbon storage and wildfowling, moderate levels of plant diversity, bird habitat and birdwatching, but very poor levels (<30\%) of bee habitat, achieving an average ES supply of $62 \%$ (Fig. 5.2). Although average ES value is a simple measure to assess multifunctionality, it cannot differentiate between sites offering many ES at a moderate level, or just one or two ES at a high level. Using Simpson's diversity index to assess ES evenness allows these effects to be disentangled, and highlights marshes offering multiple services evenly rather than one or two at high levels (Fig. 5.3). An alternative way to highlight these differences is to investigate multiple thresholds of ES delivery (Figs 5.4-5.6). These maps show that the vast majority of marshes offer at least three ES at a low level, but only one marsh offers three ES at a high level (grazing, bird habitat and birdwatching).

For all multifunctionality measures, larger marshes achieve higher values (Table 5.2), but there is no effect of distance from river mouth. These relationships are consistent with the results that marsh area has either a positive, or no effect on individual ES function, but estuary position has contrasting effects on different ES. Management type only has an association with average multifunctionality: common land and private/tenant managed land achieved higher average values than conservation/council managed marshes. All measures of multifunctionality are positively correlated with each other, with the exception of Simpson's diversity and $E S \geq 70 \%$ (Fig. 5.7), indicating that marshes can either supply a small number of services at a high level, or many services more evenly. By examining correlations between individual ES and multifunctionality measures, one can gain insight into which ES most strongly drive multifunctionality. The three ES that displayed the most significant positive correlations with multifunctionality (Fig. 5.7; grazing, 
birdwatching, wildfowling) were the three ES with the most zero values (due to no grazing, lack of public access, and prohibited shooting, respectively). ES multifunctionality could be easily increased, therefore, by targeting these 0 values, although there may be compelling reasons to keep these ES at 0 in certain marshes, such as trade-offs with other ES, or conservation or political reasons. Carbon storage exhibited a negative correlation with the Simpson's diversity measure of ES multifunctionality (Fig. 5.7), indicating that marshes high in carbon rarely provide an even supply across all ES. This correlation is perhaps due to the contrasting responses of different ES to marsh location - with some ES affected positively (including carbon storage) by distance to the river mouth, and some affected negatively (Table 5.2).

The multifunctionality measures were all analysed at the marsh scale, but because marshes have widely varying sizes, it is instructive to examine the total marsh area supplying each ES at low, moderate and high thresholds (see inserts in Figs. 5.4-5.6). From these data it is apparent that both bee habitat and wildfowling have a relatively small area of marsh supplying even low levels ( $\geq 30 \%$ ) of these two ES (Fig. 5.4 insert). As the threshold increases to a moderate $(\geq 50 \%)$ level, supply of bee habitat is particularly poor: only 138 ha of salt marsh (4\% of assessed marsh area) offers at least moderate bee habitat provision. Conversely, the majority $(>75 \%)$ of assessed salt marsh area offers at least moderate provision of bird habitat, grazing and carbon storage (Fig. 5.5 insert). Plant diversity and cultural services lie intermediate between these ES, with $20-50 \%$ of the total salt marsh area offering at least a moderate supply. These data reveal that bee habitat is a particularly under-represented ES within Carmarthen Bay, with the majority of salt marsh falling well below its maximum potential functioning level. 


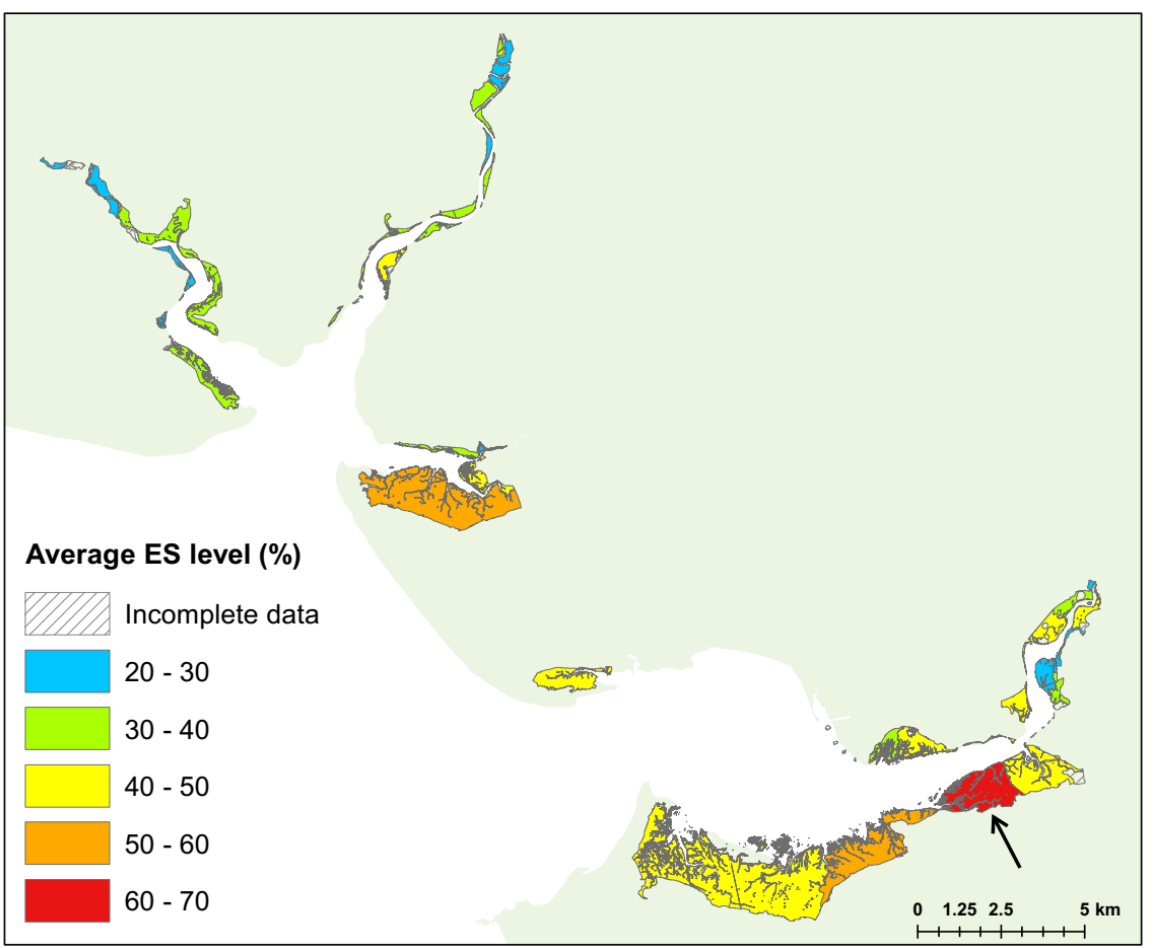

Fig. 5.2. ES multifunctionality as average ES level. The mean ES functioning level across all seven ES (after rescaling each ES as a percentage of maximum function). The arrow highlights one marsh (Penclawdd: Dalton's Point, see Tables D4-D5 for details) which is consistently amongst the top marshes for multifunctionality measures. See Fig. D3 for confidence levels.

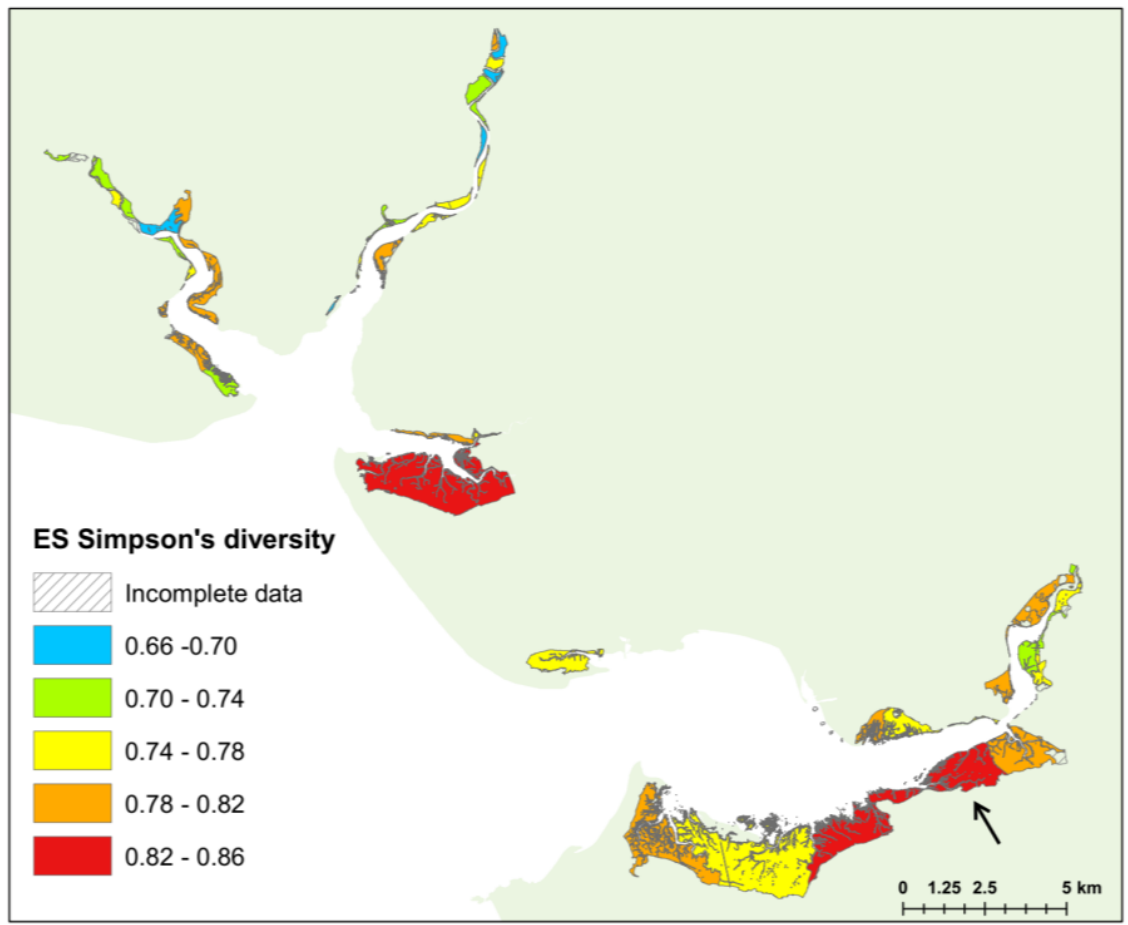

Fig. 5.3. ES multifunctionality as Simpson's diversity. The evenness of ES functioning, calculated as the Simpson's diversity index across all seven ES (after rescaling each ES as a percentage of maximum function. The arrow highlights one marsh (Penclawdd: Dalton's Point, see Tables D4-D5 for details) which is consistently amongst the top marshes for multifunctionality measures. See Fig. D3 for confidence levels. 


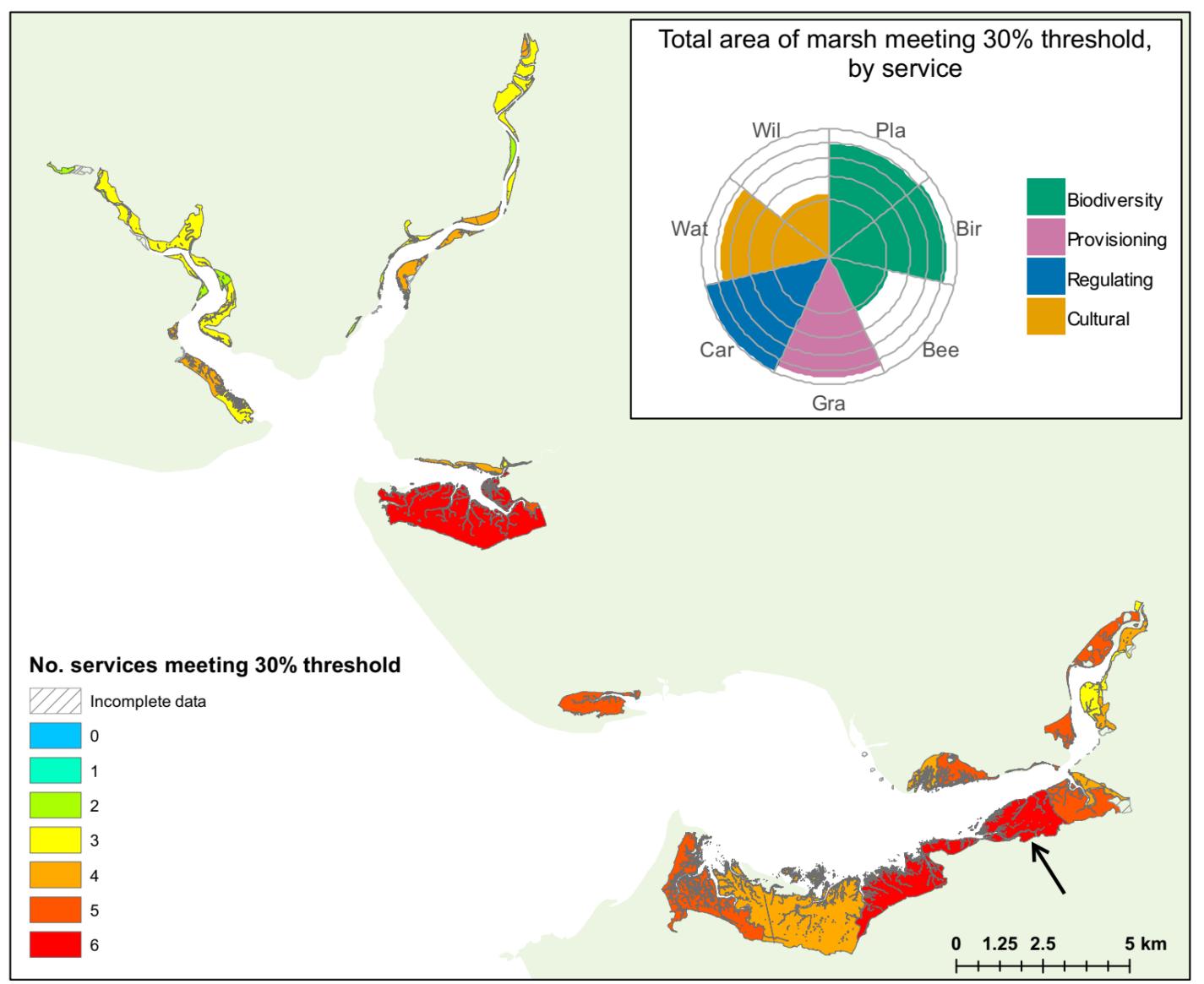

Fig. 5.4. ES multifunctionality over a low supply threshold. The total number of services (out of seven) achieving at least $30 \%$ of maximum supply. The arrow highlights one marsh (Penclawdd: Dalton's Point, see Tables D4-D5 for details) which is consistently amongst the top marshes for multifunctionality measures. Inset figure shows the total area of marsh achieving at least $30 \%$ supply level for each service: the area of slice is proportional to the area of marsh, with guides at 20, 40, 60, 80 and $100 \%$ of total assessed salt marsh area (3099 ha). See Table 5.2 legend for ES abbreviations. See Fig. D3 for confidence levels. 


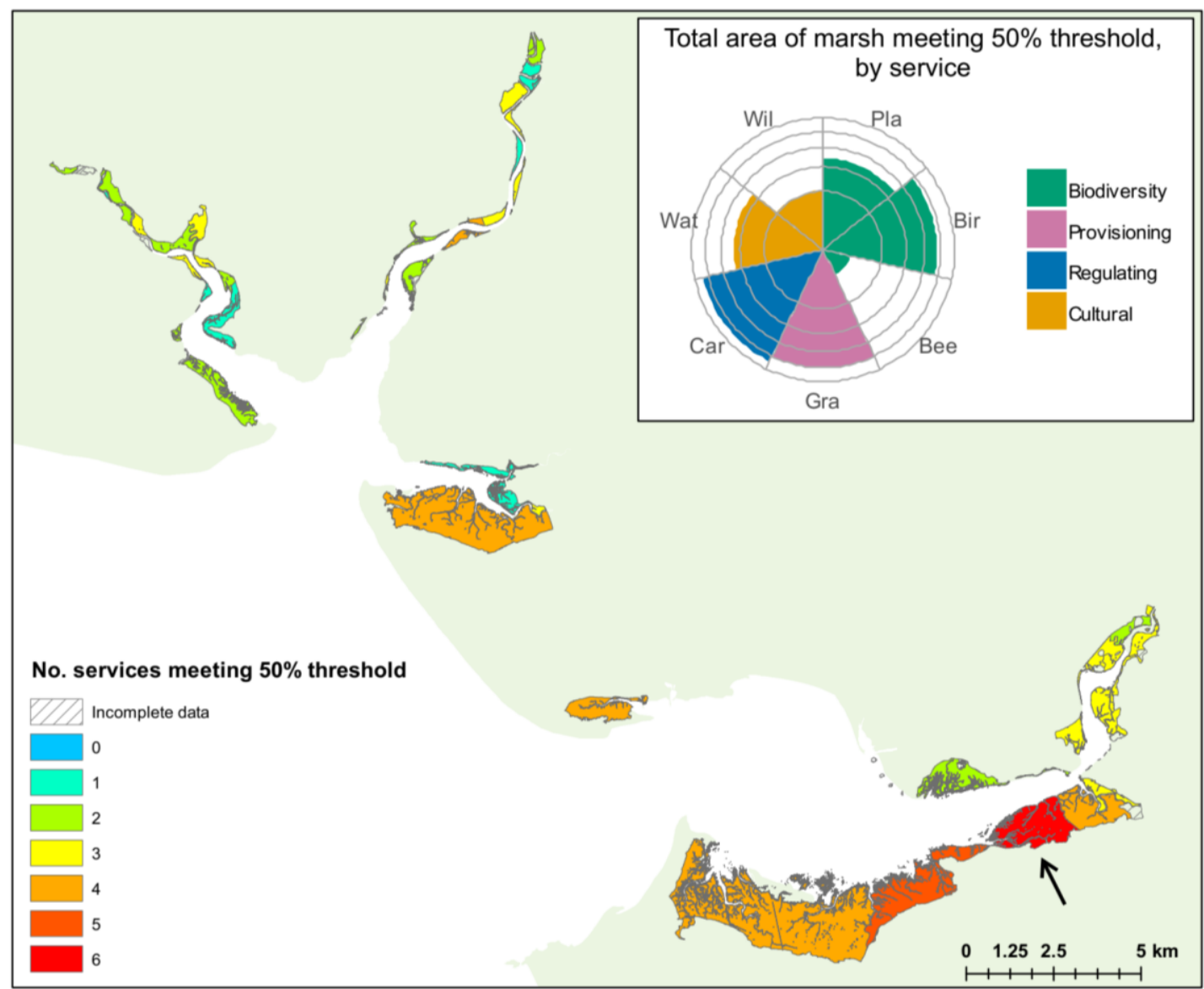

Fig. 5.5. ES multifunctionality over a moderate supply threshold. The total number of services (out of seven) achieving at least 50\% of maximum supply. The arrow highlights one marsh (Penclawdd: Dalton's Point, see Tables D4-D5 for details) which is consistently amongst the top marshes for multifunctionality measures. Inset figure shows the total area of marsh achieving at least $50 \%$ supply level for each service: the area of slice is proportional to the area of marsh, with guides at 20,40,60, 80 and $100 \%$ of total assessed salt marsh area (3099 ha). See Table 5.2 legend for ES abbreviations. See Fig. D3 for confidence levels. 


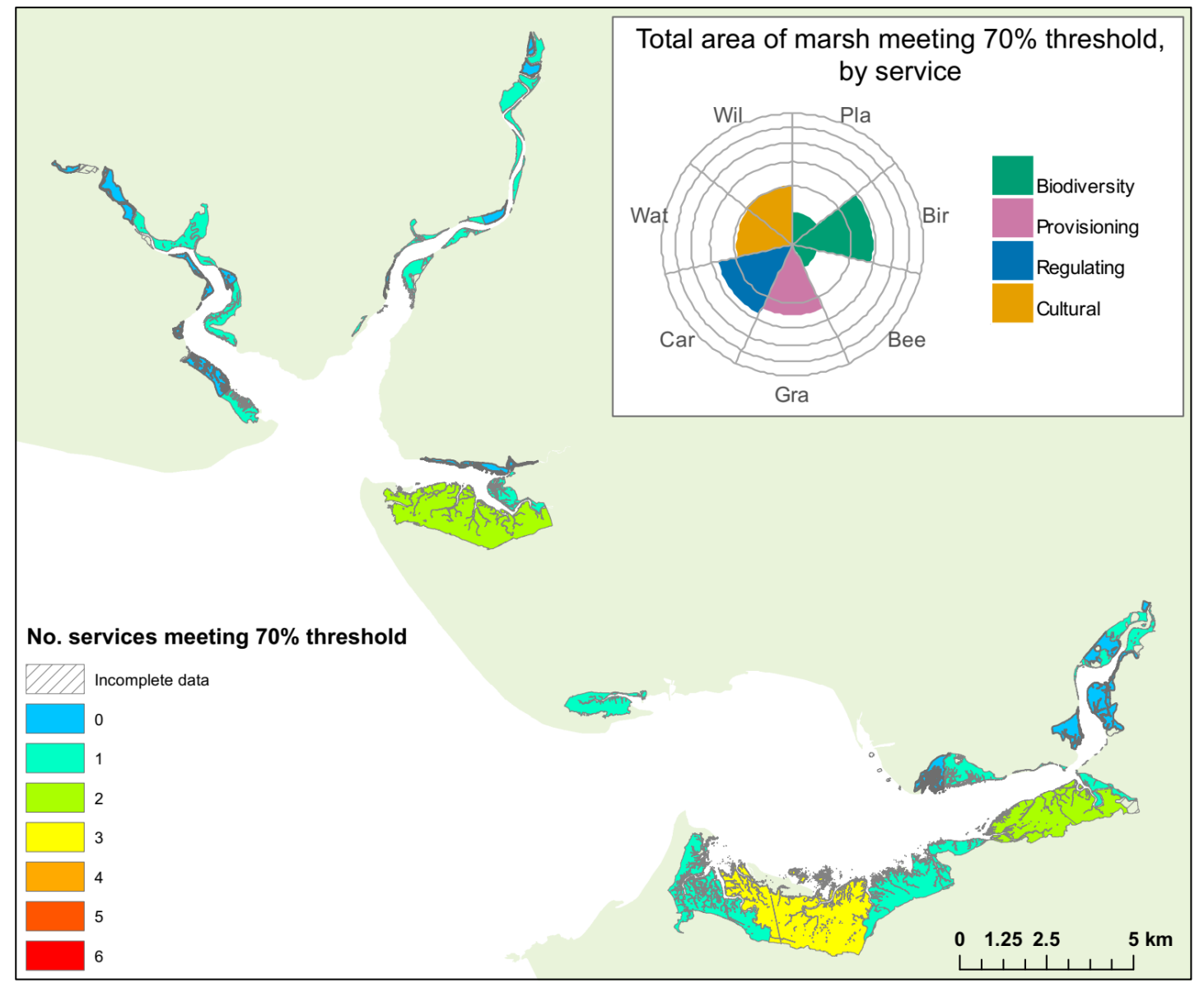

Fig. 5.6. ES multifunctionality over a high supply threshold. The total number of services (out of seven) achieving at least $70 \%$ of maximum supply. Inset figure shows the total area of marsh achieving at least $70 \%$ supply level for each service: the area of slice is proportional to the area of marsh, with guides at 20,40,60, 80 and $100 \%$ of total assessed salt marsh area (3099 ha). See Table 5.2 legend for ES abbreviations. See Fig. D3 for confidence levels.

\section{Interactions among services and ecosystem service bundles}

I tested the spatial correlation between pairs of ES, to gain insight into potential trade-offs and synergies (Fig. 5.7). There were no significant positive correlations between any pairs of ES (indicative of synergies) but two significant negative correlations (indicative of trade-offs). This result accords with a meta-analysis of ES interactions that showed ES trade-offs are three times more common than ES synergies (Howe et al. 2014). Grazing and bee habitat exhibit spatial trade-offs, which is unsurprising, as grazing level was a key variable used to predict bee habitat value. Grazing directly drives a reduction in bee habitat value via reductions in the flower cover of key food plants (Chapter 4). Bird habitat and carbon also exhibited 
spatial trade-offs, although the reason for this is not obvious. This is likely to be a result of both ES responding in contrasting ways to a common driver, such as plant community: one of the plant communities with the highest carbon values - SM18 - is dominated by the long, unpalatable Juncus maritimus, whereas grazing saltmarsh birds prefer a short, palatable sward (Bos et al. 2005).

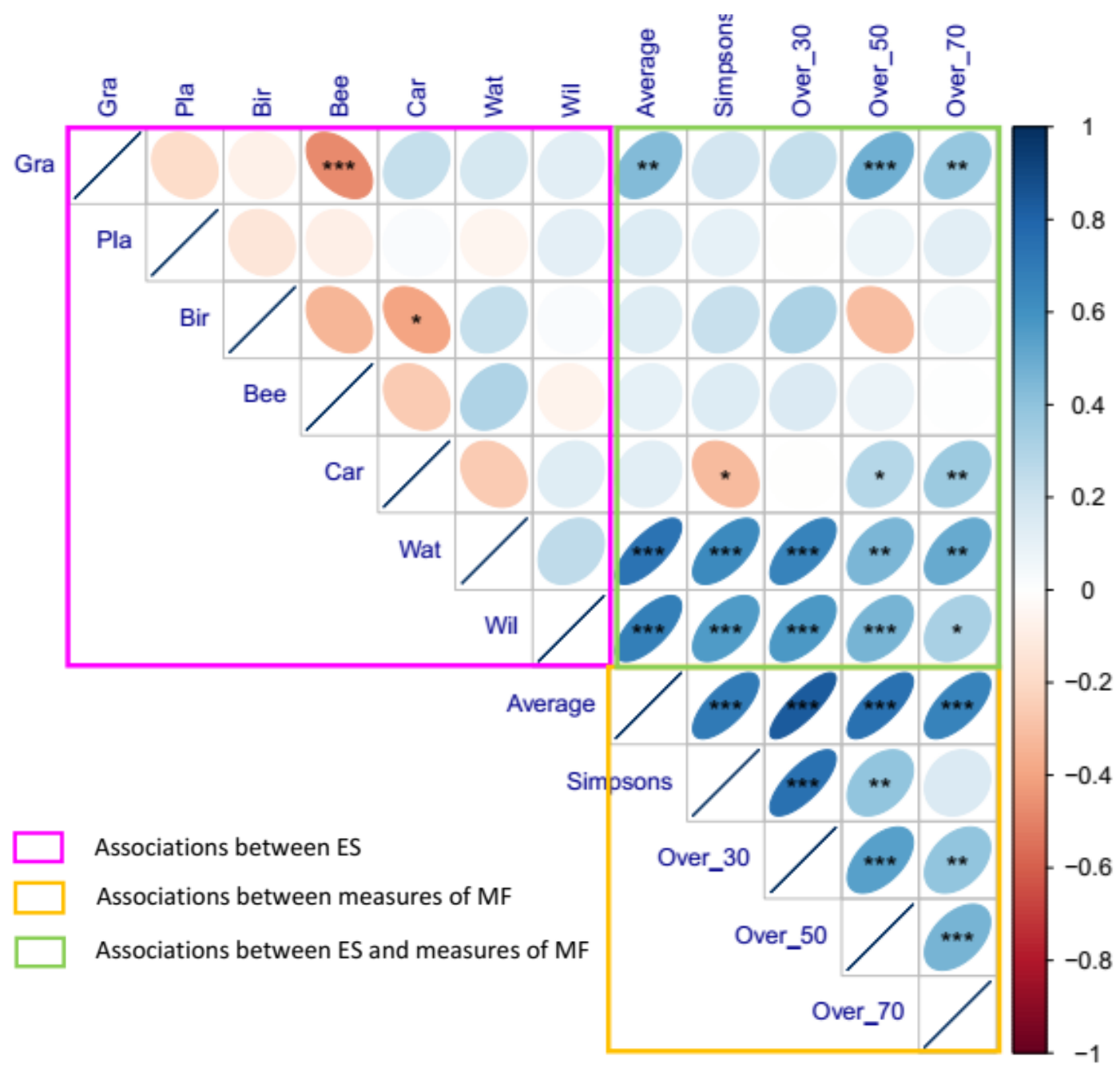

Fig. 5.7. Correlations between ES and multifunctionality measures. Ellipses show positive (blue) and negative (red) correlations between pairs of ES (pink box), between pairs of multifunctionality (MF) measures (yellow box), and between individual ES and MF measures (green box). The narrowness of the ellipse and depth of the colour indicates the size of the correlation coefficient. Significant results from Pearson's correlation tests are indicated with asterisks, with significance levels of $0.05\left({ }^{*}\right), 0.01$ $\left({ }^{* *}\right)$, and $0.001(* * *)$. Pla=plant diversity, Bir=bird habitat, Bee=bee habitat, Gra=grazing, Car=carbon storage, Wat=birdwatching, Wil=wildfowling. 
I identified six ES bundles across Carmarthen Bay salt marshes, representing marshes displaying similar type and magnitude of ES. Six was the optimum number of bundles identified by silhouette analysis (i.e. analysis of the distance between clusters), which is high but not unusual compared to other studies, which typically produce 3-7 bundles (e.g. Raudsepp-Hearne et al. 2010; Martín-López et al. 2012; Turner et al. 2014; Renard et al. 2015; Spake et al. 2017; Lin et al. 2018). I used Principal Components Analysis (PCA) to explore patterns in ES provision (Fig. 5.8a). Four PCs are required to describe $>80 \%$ of cumulative variance, indicating fairly high dimensionality. PC1 explained 32\% and corresponded to an axis that varied from high value bee habitat to high value grazing land. This axis aligns with the most highly significant ES trade-off that was identified via correlation analysis (i.e. between grazing and bee habitat). PC2 explained $24 \%$ and corresponded to an axis that varied from high value grazing and carbon to high value bird habitat, wildfowling and birdwatching. The trade-off between carbon and bird habitat was also identified via correlation analysis.

Where trade-offs and synergies between ES are strong, one would expect to see many significant correlations between services (e.g. Raudsepp-Hearne et al. 2010) and low dimensionality of ES producing a low number of bundles, each dominated by a small number of ES (e.g. Spake et al. 2017). The small number of significant correlations between ES, high dimensionality and high number of bundles identified here, indicates that strong trade-offs or synergies are not common within this system. This may make the system complex to manage, as there are few opportunities to drive changes in ES provision. However, examination of the levels of ES supplied by each bundle (Fig. 5.8b), the marsh area providing each bundle (Fig. 5.9), and the characteristics of the marshes providing each bundle (Table 5.3), allows an understanding of the types of ES supply within Carmarthen Bay salt marshes, and highlights any over- or under-representation of bundles. 


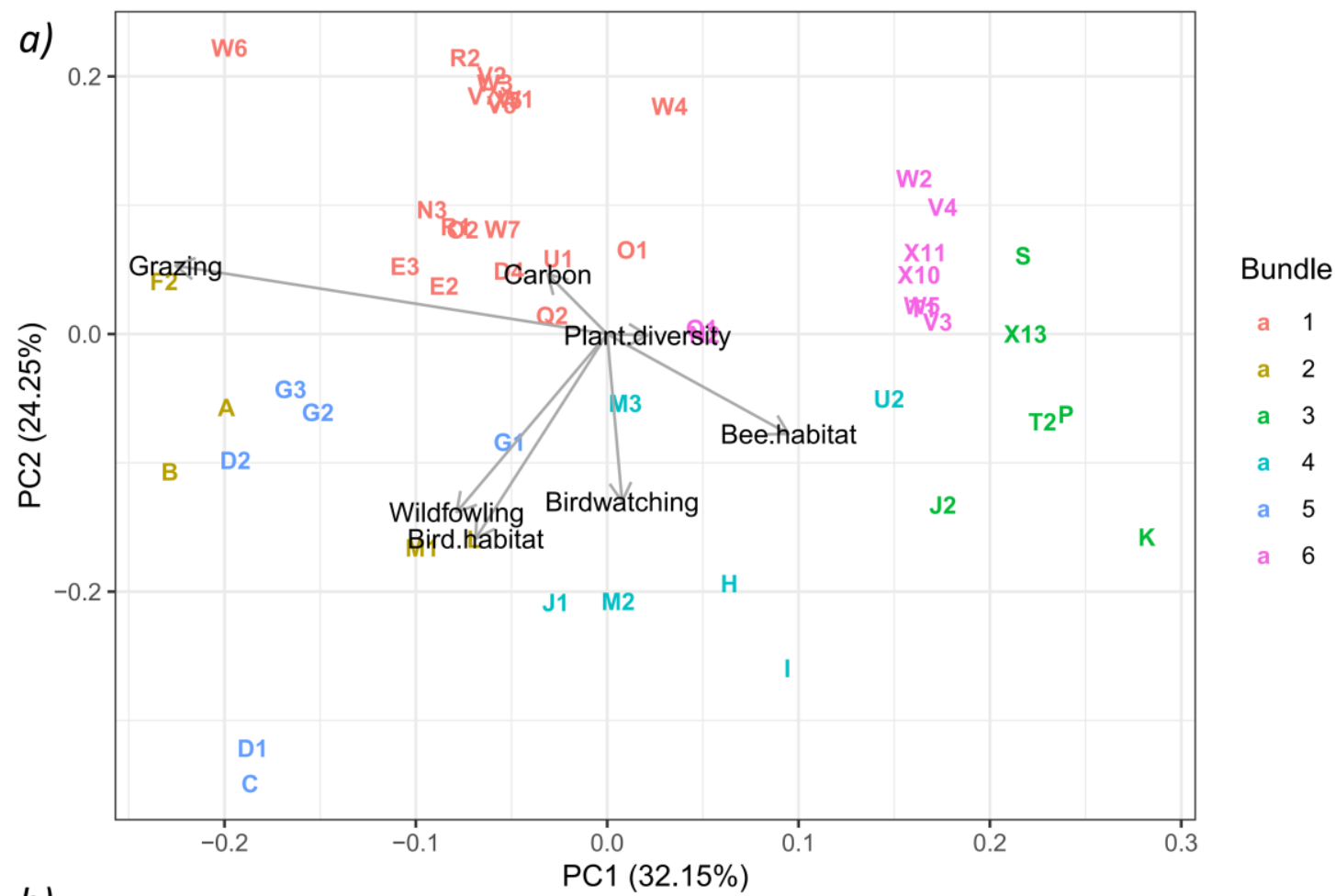

b)

1 Grazing, no recreation

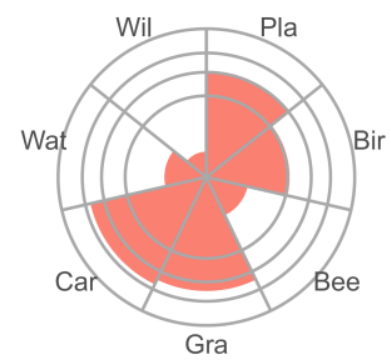

4

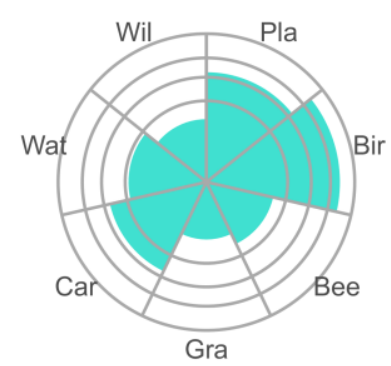

2

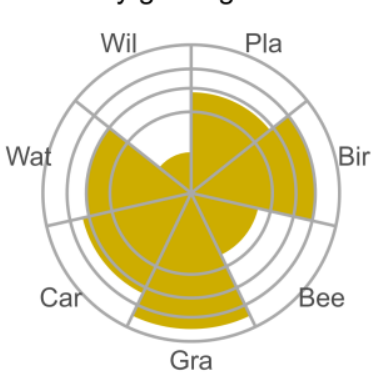

5

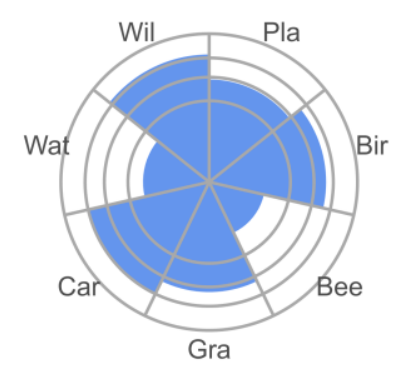

3 undle

1

2

3

5

6

Fig. 5.8. Ecosystem service (ES) bundles within the study area. a) Clustering of the six ES bundles along the first two axes obtained from Principal Components Analysis. $b$ ) The average ES supply within each bundle: the area of slice is proportional to the percentage of maximum functioning for each ES, with guides at 30 (low supply), 50 (moderate supply), 70 (high supply) and 100\% of maximum functioning. See Table 5.2 legend for ES abbreviations. Pla=plant diversity, Bir=bird habitat, Bee=bee habitat, Gra=grazing, Car=carbon storage, Wat=birdwatching, Wil=wildfowling. 


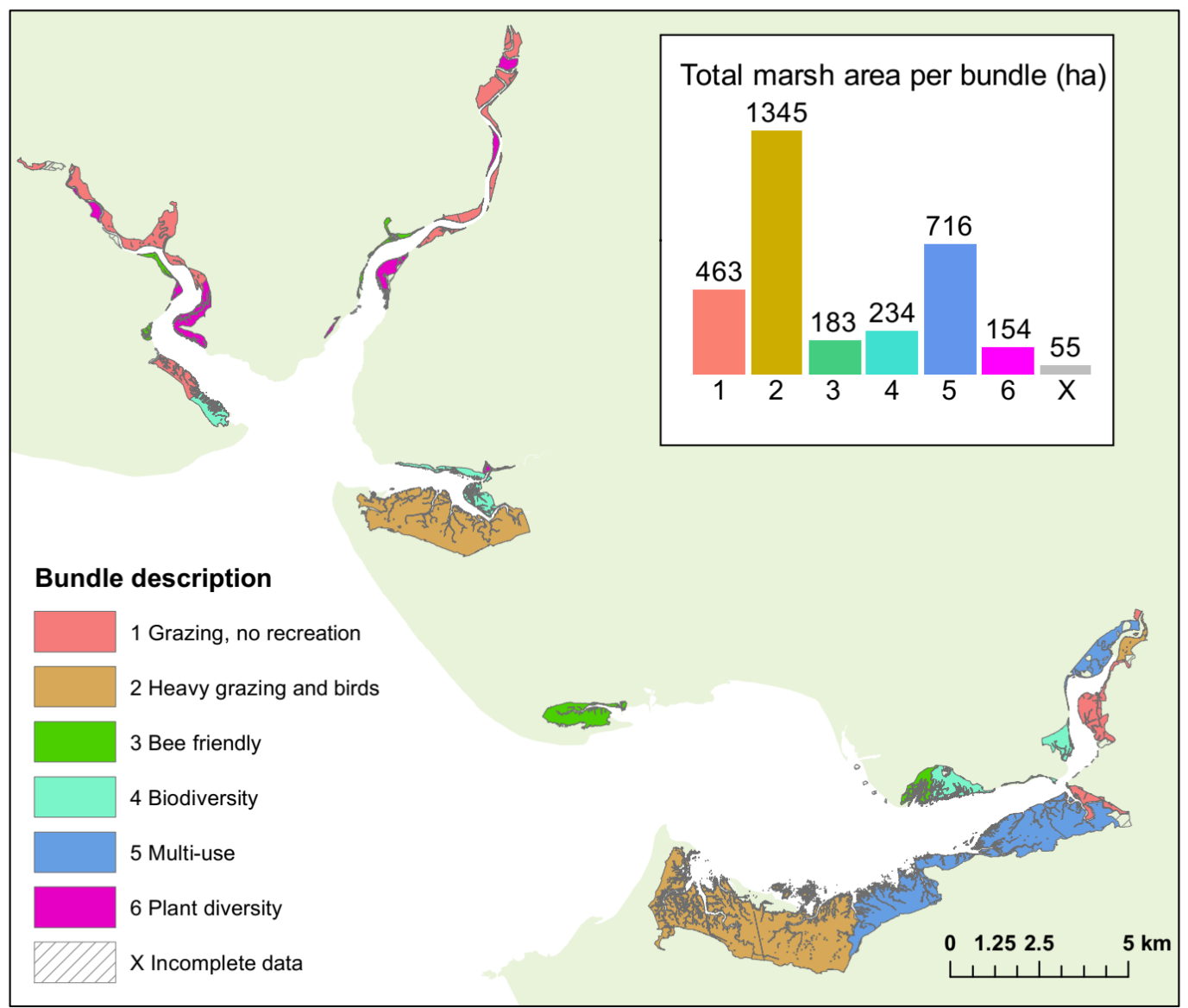

Fig. 5.9. Distribution of ecosystem service bundles across the study area. The inset figure shows the total area of salt marsh falling within each bundle. See Fig. D3 for confidence levels.

Bundle 1 is the most common bundle, and comprises marshes that offer fairly high values for grazing, but extremely low values for recreation in the form of birdwatching or wildfowling. Bundle 2 contains the most heavily-grazed marshes, providing high value habitat for overwintering birds and also moderate birdwatching values. The large marshes in this bundle cover $43 \%$ of the total marsh area. Bundle 3 represents the six marshes that are ungrazed and offer good bee-habitat, and covers just $6 \%$ of the salt marsh area. Bundle 4 contains marshes that offer moderate and high supplies of plant diversity and bird habitat. Bundle 5 contains marshes that offer five of the seven ES at moderate or high levels, so I have classified these marshes as multi-use. Both plant diversity and carbon are fairly equitably spread across all the bundles: neither service falls short of a low supply threshold in any bundle. However, plant diversity is highest in bundle 6 , which also offers moderate supply of carbon 
storage but poor supply of all other ES. The nine small marshes within bundle 6 covers just $5 \%$ of the total area.

Bundles that offer high $(\geq 70 \%)$ or moderate $(\geq 50 \%)$ levels of grazing are particularly well-represented in terms of total salt marsh area (bundles 1,2,5: $82 \%$ of assessed area). However, only a single bundle offers bee habitat exceeding a low supply threshold (bundle 3 ) and this covers just $6 \%$ of the salt marsh area. These two ES display the clearest spatial trade-off, and the strong over-representation of livestock grazing is resulting in an under-representation of good quality bee habitat. In contrast, moderate or high levels of grazing can co-exist with moderate or high levels of plant diversity (bundle 1), bird habitat (2 and 5), carbon storage $(1,2,5)$, birdwatching (2), and wildfowling (5). Although birdwatching and wildfowling only exceed low levels within one bundle each ( 2 and 5, respectively), these bundles cover large areas of salt marsh (43 and $23 \%$ of total area, respectively) so these ES are not as under-represented as bee habitat. However, both these cultural services have better supply/availability within the Loughor estuary than the Three Rivers estuary. Correlation analysis indicated a spatial trade-off between bird habitat and carbon storage. However, two bundles ( 2 and 5 ) supply both bird habitat and carbon storage at moderate levels or higher, showing that trade-offs between these services are not inevitable.

Table 5.3. Marsh characteristics for each ES bundle. Total area shows the summed area of all marshes supplying each ES bundle. Management shows the count of marshes falling under each management type: $\mathrm{C}=$ common land with multiple graziers, $\mathrm{CC}=$ conservation body or council, $\mathrm{PT}=$ private owner or tenant farmer, NK=not known.

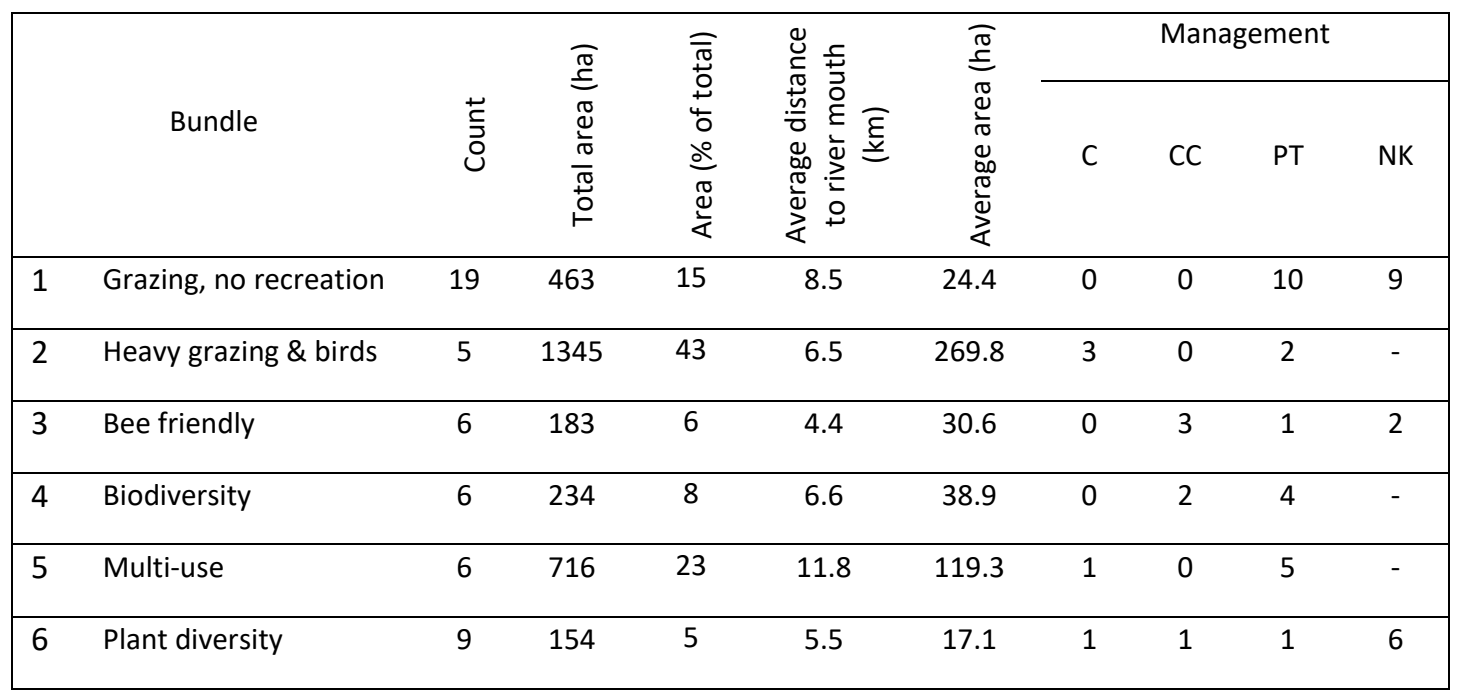




\subsubsection{Data limitations and recommendations for future study}

Each ES map layer has its own limitations, which are described in Appendix D. More general limitations also apply. ES were selected based on the availability of sitespecific, relevant data. It was not within the scope of this project to map several important services such as coastal protection and breeding bird habitat. Coastal protection is an important service provided by salt marshes, derived from wave attenuation caused by aboveground biomass (Möller et al. 1999; Arkema et al. 2013). Wave attenuation may be impaired by grazing, due to reduction in the canopy height (Möller et al. 2014), but modelling this ES is complex and requires accurate bathymetry data. Breeding birds generally prefer a structurally diverse sward with longer vegetation, and their nests are at risk from trampling by grazers (Norris 2000; Sharps et al. 2015). Because the Special Protected Areas were designated for overwintering birds, the overwintering bird populations are more relevant for this case study, but the lack of ungrazed and lightly-grazed marshes within the study area may severely limit its potential as a breeding bird habitat, so spatial analysis of this ES would be desirable. There is the potential for grazing livestock and waterfowl to have strong negative effects on coastal water quality, via contamination by faecal matter washed off on ebbing tides (Jones and Obiri-Danso 1998). This could create a trade-off between livestock grazing/bird habitat and cockle fisheries/bathers within Carmarthen Bay. Field investigations and modelling are needed to assess and quantify this trade-off.

Several of the assessed ES are linked, but this is a natural consequence of a complex system, and each ES is also influenced by other variables and used by different stakeholders: high value bird habitat supports both birdwatching and wildfowling, but each of these end user groups is affected by other factors (e.g. the presence of a hide for birdwatchers; the availability of licenced shooting for wildfowlers). Bee habitat was predicted by grazing levels and plant diversity, but also by the presence of key bee-friendly plant species. Some of the measures used here are for realised benefits (e.g. bag counts for wildfowling) so take account of both supply and demand for the service, while some are for potential supply (e.g. birdwatching) and do not account for demand, but the valuation measures selected were the best available, considering time and data constraints. For the purpose of normalising different 
measures of ES supply, I assumed that the maximum value scored within the study area is the maximum potential value, and, for ES where 0 is never achievable (carbon storage, plant diversity), that the minimum recorded score is the minimum potential value. However, these figures are relevant at the site level only, so cannot be used to infer ES delivery at a national or international scale. Extending this work to other Welsh and European estuaries would allow an understanding of saltmarsh ES provision within a national and international context. The multifunctionality assessments present each ES as having equal importance and desirability, but this does not represent different stakeholder interests. Stakeholder engagement work is necessary to allow ES to be weighted by importance, so that the performance of each marsh, and the entire landscape, can be assessed in relation to different stakeholder priorities and conservation objectives (Manning et al. 2018).

\subsection{Conclusions and management implications}

Here, for the first time, multiple ES have been mapped for salt marshes, allowing identification of trade-offs, highlighting areas of concern, and informing management of a complex site that serves multiple stakeholders. I have shown that different forms of management are associated with different ES: grazing and birdwatching values are highest on common land, bee habitat is highest on conservation body/local council managed marshes, and carbon storage is highest on private/tenant managed marshes. These varying responses of individual ES mean that no single management type is clearly optimal for overall ES multifunctionality, although common land does produce the highest average ES function level. Three of the seven ES (grazing, birdwatching, wildfowling) increase with increasing marsh area, leading to marsh area having a positive effect on ES multifunctionality. Different ES exhibit different responses to estuary position: displaying both positive (grazing, carbon storage) and negative (plant diversity, bee habitat) relationships with distance from river mouth.

There were few correlations between services. The clearest ES trade-off is between a provisioning service - grazing - and a biodiversity/supporting service - bee habitat. This trade-off is due to a direct effect of grazing on bee habitat quality (Chapter 4). 
Analysis of ES exceeding multiple thresholds revealed that only a small area of marsh ( $4 \%$ of total) provides even a low supply of bee habitat, and analysis of ES bundles showed that a moderate supply of bee habitat is only achieved when grazing is absent. Due to the economic incentive for livestock grazing, it is not surprising that it is primarily conservation body/council managed marshes that are maintained without grazers, to the benefit of bees. In order to increase the coverage of beefriendly habitat, land managers are likely to need economic incentives to reduce grazing levels, in the form of Agri-Environment Scheme payments. Correlation analysis showed that bird habitat trades-off against carbon, but both services cooccur at moderate levels in multiple bundles, indicating that this trade-off is not strong or inevitable, and is likely due to both ES reacting differently to a common driver, such as plant community.

Apart from bee habitat, the most spatially-restricted ES are the cultural services of birdwatching and wildfowling, which can have values of zero due to a lack of public access or a lack of licenced shooting, respectively. High values of birdwatching and wildfowling are generally found in larger marshes, meaning that the total area of marsh providing these ES is relatively high. However, because the largest marshes are found to the southeast of the study area, stakeholders in the northwest have a lower supply of these cultural services. There are licenced shooting grounds along all rivers in the study area, so the lower supply of wildfowling in the northwest may be partly due to lower demand. Due to possible conflicts between wildfowlers and conservation objectives and other marsh users (Owen 1990), there is little incentive to increase the coverage of licenced shooting areas. However, the lower supply of birdwatching in the northwest is primarily due to restricted access. Birdwatching value could be increased here by increasing accessibility to sites with footpaths, and increasing the availability of bird hides, which are valued by birdwatchers and would minimise the impact to wildlife of increasing visitor numbers.

Through this case study, i have shown that a single land cover type - salt marsh cannot be considered a homogenous ES provider. Within this habitat, variation in management, size, location, and vegetation community is associated with varying provision of ES and ES multifunctionality, leading to the supply of multiple distinct ES 
bundles. Land cover type is commonly used to predict ES provision, due to the ready availability of land cover maps, and is the most convenient way to assess multiple ES across landscapes. This is the first study to explicitly map multiple ES in salt marshes, and the values are transferrable to other ES assessments covering temperate European salt marshes. Due to the wide variation exhibited within this study, I recommend increased research into the true range of ES supply from individual habitats, and the factors that influence this. This will allow increased accuracy in the assessment of ES trade-offs and the likely impact of land-use or land-cover changes across landscapes.

\subsection{Acknowledgments}

Thanks to Lily Pauls, Heather Lewis, Kerry Rogers, Ruth Harding and Richard Facey at Natural Resources Wales for providing access to data and contextual information for the site. Thanks also to Davide de Battisti (Swansea University) for sharing his soil carbon data, the wildfowling clubs who provided bag returns, experts who responded to our birdwatching survey, and farmers/landowners who took the grazing survey.

\subsection{Supporting Information}

The following supplementary material can be found in Appendices D-F. Appendix D: Supporting information for Chapter 5:

Supplementary methods - Calculating Ecosystem Service values.

Table D1. Linear model results for optimal predictive model of bee habitat value.

Table D2. Predicted carbon stock in top $10 \mathrm{~cm}$ of saltmarsh sediment by NVC class.

Table D3. Features contributing to overall birdwatching value.

Table D4. Marsh attributes and raw ecosystem service (ES) values for all assessed marshes.

Table D5. Rescaled ecosystem service values, multifunctionality scores and ES bundles for all marshes.

Fig. D1. Heat maps of individual ES provision. 
Fig. D2. Confidence in each ES map layer.

Fig. D3. Confidence levels for ES multifunctionality values and ES bundles. Appendix E: Owner/occupier interviews of saltmarsh management. Appendix F: Expert opinion birdwatching survey. 


\section{Chapter 6. Thesis discussion}

\subsection{Overview of results}

Ecosystem service (ES) studies that consider multiple services, and their interactions, are relatively rare (Boerema et al. 2016). In this thesis I have used a variety of approaches to investigate the impacts of livestock grazing on multiple saltmarsh ES (see Table 6.1 for a summary of the results). A systematic review of the academic literature (Chapter 2) revealed that research into grazer impacts on salt marshes is dominated by European studies. Although grazing is extremely common in Europe, this heavy weighting of European studies creates a risk that a consensus of grazer impacts is reached based on studies from a single region, highlighting a need for more data from outside Europe. The meta-analysis revealed some consistent effects of grazers on soil, vegetation, and fauna properties. The largest effect sizes were seen with vegetation responses (reduced height, aboveground biomass and litter) and herbivorous insects (reduced abundance). 'Conservation grazing' is commonly applied to salt marshes, and this meta-analysis confirmed that grazing increases plant richness, but there were also reductions in invertebrate richness with grazing. Grazing did not impact the carbon in European salt marshes, but it did reduce the carbon stored in American salt marshes.

Surveys of grazed and ungrazed marshes along the southeast US coast (Chapter 3) strengthened this result, using standardised survey techniques and accounting for soil texture and other covariates to show that soil carbon is significantly reduced in grazed marshes in America. The observational results indicate that grazing shifts the plant community to a more diverse community with reduced Spartina dominance. The experimental study on Cumberland Island (Chapter 3 ) showed that this change in 
plant community is relatively rapid - significant differences appeared after just 13 months of grazer exclusion. However, effects of grazing on soil carbon were not yet apparent after 18 months of exclusion. The US study concurred with the metaanalysis in showing strong effects of grazing on vegetation properties. In contrast to the meta-analysis, there were no significant changes to soil salinity or redox, and primary consumers in southeast ES salt marshes were robust to competition from large herbivores - only adult fiddler crabs benefitted from horse exclusion in the experimental study and long-term grazing had no effect on snails, crabs or mussels in US marshes. The experimental study showed no clear impact of grazing on saltmarsh resilience, although a longer period of grazer exclusion may be necessary for changes to take effect.

The meta-analysis in Chapter 2 showed that invertebrates are generally disadvantaged by grazing, both in terms of abundance and diversity. In Chapter 4 I focussed on one specific interaction, between grazing and bee habitat value, allowing an understanding of the precise mechanisms underpinning this trade-off. Surveys of 11 Welsh marshes showed that grazing reduced flower cover of the two most important plants for foraging bees, and that increases in plant diversity with grazing did not compensate for these negative effects. Comparisons with terrestrial surveys revealed that when not grazed, salt marshes can be a highly valuable foraging ground for honeybees and bumblebees, providing vital nectar and pollen resources in mid to late summer.

Through mapping the spatial distribution of multiple saltmarsh ES in a UK estuary complex (Chapter 5), I showed that the supply of some saltmarsh ES can be highly variable, and supplies vary with saltmarsh management, size and location. Spatial analysis revealed that strong interactions between ES are rare, and it is possible for a marsh to supply six out of seven assessed services over a moderate supply threshold. The only strong trade-off associated with grazing was the one revealed in Chapter 4, between grazing and bee habitat. Spatial analysis revealed that, within Carmarthen Bay, salt marshes are not realising their potential as a bee foraging habitat, due to the prioritisation and predominance of grazing in this area. However, due to a lack of available spatial data, several important ES services that may conflict with grazing, 
such as breeding bird habitat, coastal protection and water quality, were not assessed.

Table 6.1 Summary of major grazer effects on ecosystem properties and their related services (ES). Arrows show whether the property increases or decreases with grazing, and how these changes are likely to impact on ES provision. In general, I have not included properties that do not change with grazing $(\leftrightarrow)$, except where the results from different studies or regions are inconsistent.

\begin{tabular}{|lll|}
\hline Property & Ecosystem service & Chapter(s) \\
\hline$\uparrow$ Plant richness & $\uparrow$ Biodiversity (S) & $2,3,4$ \\
\hline$\downarrow$ Invertebrate richness & $\downarrow$ Biodiversity (S) & 2 \\
\hline $\begin{array}{l}\text { S Salinity, temperature, bulk density } \\
\downarrow \text { Redox }\end{array}$ & $\downarrow$ Nutrient cycling (S) & 2 \\
\hline$\leftrightarrow$ Salinity, redox & $\leftrightarrow$ Nutrient cycling (S) & 3 \\
\hline$\downarrow$ Cover of Aster and Limonium & $\downarrow$ Bee habitat (S) & 4 \\
\hline$\leftrightarrow$ Soil carbon (Europe) & $\leftrightarrow$ Carbon storage (R) & 2 \\
\hline$\downarrow$ Soil carbon (America) & $\downarrow$ Carbon storage (R) & 2,3 \\
\hline$\downarrow$ Plant biomass, height, cover & $\downarrow$ Coastal protection (R) & 2,3 \\
& $\downarrow$ Fisheries (P) & 2 \\
\hline$\downarrow$ Invertebrate abundance (global) & $\downarrow$ Fisheries (P) & 3 \\
\hline$\leftrightarrow$ Invertebrate abundance (US)* & $\leftrightarrow$ Fisheries (P) & 2 \\
\hline$\uparrow$ Vertebrate abundance (long-term grazing) & $\uparrow$ Wildfowling (P,C) \\
& $\uparrow$ Birdwatching (C) & \\
\hline$\leftrightarrow$ Vertebrate abundance & $\leftrightarrow$ Wildfowling (P,C) \\
& $\leftrightarrow$ Birdwatching (C) & \\
\hline
\end{tabular}

* Response from adult fiddler crabs in experimental plots only

\section{Management implications}

These results provide evidence for four main management recommendations.

1. There is no one-size-fits-all optimal grazing regime for salt marshes. Managers must take account of local ES demands and conservation priorities.

2. Plant diversity should not be used as a proxy for overall ecosystem diversity.

3. Long-term ungrazed marshes are a rare habitat in the UK, and are a valuable habitat for bees, therefore these marshes should be protected from any grazing. The cover of bee-friendly habitat could be increased by reducing grazing levels in intensively grazed marshes.

4. Grazing in American salt marshes should not be widely practiced, due to the impact on globally important stores of 'blue carbon'. However, the current horse and cattle grazing in southeast US is not a major concern, due to its restricted 
distribution, and apparently minimal impact on saltmarsh fauna, multifunctionality and resilience.

\subsection{Saltmarsh grazing in context}

Salt marshes are unique as grazing lands, due to their liminal quality - sometimes land, sometimes sea. The necessity of removing livestock during spring tides means that graziers cannot rely on salt marshes exclusively. Salt marshes can be fickle, expanding or contracting over time, and shifting from one side of an estuary to the other (Ladd et al. in press). There are natural limits on livestock density: networks of creeks often restrict access to large areas of marsh, and the high soil moisture would cause soils to become completely churned up under very heavy grazing. Therefore, the heaviest levels of grazing recorded in salt marshes (all except two studies in my meta-analysis reported $\leq 2$ livestock units $\mathrm{ha}^{-1}$ ) are lower than levels in other systems (2 LSU ha-1 is the average for lowland grazing in England, Natural England 2009; Finch et al. 2014 consider >3 LSU ha ${ }^{-1}$ to be heavy grazing). These relatively limited grazing levels may explain why I discovered few trade-offs in Chapter 5, compared to the frequent, strong trade-offs between agriculture and other ES reported from terrestrial systems (e.g. Alkemade et al. 2013; Petz et al. 2014; Chillo et al. 2016; Holt et al. 2016; Johansen et al. 2019). However, it is difficult to fully assess this, as most studies report descriptive, relative levels (e.g. extensive and intensive, under-grazed and over-grazed) rather than absolute quantitative values such as LSU ha-1. The difficulty of quantifying grazing levels in an unfenced rangeland was described in Chapter 4, and besides, the carrying capacity of a rangeland will vary depending on habitat productivity (Ebrahimi et al. 2010).

Another factor limiting the impact of grazing on other ES in salt marshes may be the lack of agrochemicals in this system. Agrochemicals drive many of the trade-offs between agriculture and ES (Holt et al. 2016), but in salt marshes, regular tidal flooding contributes to soil fertility and washes off pathogens, meaning agrochemicals are rarely applied (Chatters 2004). However, tidal flooding may drive negative impacts of saltmarsh grazing on neighbouring coastal waters, by washing off animal excrement (S. Thomas, Natural Resources Wales, 16 May 2017, personal 
communication). Faecal contamination of rivers from terrestrial grazing lands is fairly well understood (reviewed by Murphy et al. 2015), but contamination pathways and severity for grazed salt marshes have not yet been investigated.

It is difficult to make generalisations about the effects of grazing on terrestrial rangelands, as grazer impacts are moderated by many variables, including grazer type and intensity, evolutionary history of grazing, plant diversity, productivity and functional traits (Török et al. 2018). Many of the results found in my meta-analysis (Chapter 2) - reductions in plant biomass, cover and litter, shifting plant community composition, changes to soil structure and function - are consistent with results from terrestrial studies (reviewed by Török et al. 2018) and other coastal grasslands (Ford et al. 2012b). Terrestrial studies have also shown that the effect of grazers on carbon storage is complex, moderated by variables including climate, sediment type and plant type (McSherry and Ritchie 2013; Abdalla et al. 2018). However, a clear understanding of the factors moderating grazer impacts will only be gained by mechanistic study of the processes operating in each system.

A study of multiple ES supplied by semi-natural grasslands in Norway found that sheep grazing had a significant effect on all six measured ES except pollination (Johansen et al. 2019). This contrasts with my studies, where the clearest trade-off I identified was between grazing and bee habitat (Chapter 4). Studies in terrestrial habitats have displayed mixed results of grazing on pollinators (reviewed by Lázaro et al. 2016). The strong trade-off in salt marshes may be driven by the functional composition of salt marsh plant communities. I found that just two plants supplied the vast majority of bee foraging resource - these were both reduced by grazing and there were no functionally similar plants to take their place. Usually, plant-pollinator networks are more complex, with pollinators relying on a much broader suite of flowering plants (Memmott 1999) and this higher diversity in terrestrial systems may confer increased functional resilience (Griffin et al. 2009).

Terrestrial rangelands may gain benefits from grazing that are not present for salt marshes. Grazing can suppress encroachment by shrubs and trees (Newton et al. 2009), and the subsequent loss of the ES associated with open habitats. However, the environmental stress of regular tidal flooding restricts the plants that can grow 
on a salt marsh (Adam 1990), so grazing is not needed to arrest succession here. In other circumstances, tidal flooding may mitigate the effects of grazing on salt marshes: as well as restricting grazer densities, flooding helps to introduce and redistribute organic matter around a salt marsh (Adam 1990). This may explain why herbivorous invertebrates are so robust to grazer impacts in highly productive US marshes (Chapter 3), while consumers in terrestrial systems are generally suppressed by the presence of large herbivores (Daskin and Pringle 2016).

Environmental stress from tidal flooding will increase with rising sea levels (Gedan et al. 2009). Although salt marshes can accrete vertically as sea-levels rise, this capacity is dependent on sufficient sediment supply (Kirwan et al. 2016; Schuerch et al. 2018). Livestock may interact with this accretion via soil compaction (Chapter 2), but there is mixed evidence as to whether this is overall harmful for saltmarsh accretion, by reducing elevation (Elschot et al. 2013), or beneficial, by reducing erosion (Pagès et al. 2018). The relative importance of grazers relative to sediment supply is unknown, but may differ between minerogenic European marshes and organogenic American marshes (Bakker et al. 2015). A potentially greater problem is the limited capacity for horizontal expansion - many salt marshes may be unable to migrate inland due to steep shorelines or coastal infrastructure such as roads and sea walls (Gedan et al. 2009; Schuerch et al. 2018). This 'coastal squeeze' between rising seas and inland boundaries will reduce the supply of all saltmarsh ES, including livestock grazing. However, the economic value of salt marsh as pastureland provides an extra weapon in the armoury to persuade policy-makers to allow inland migration of salt marshes if farmers and landowners can use these tidal lands profitably, they are less likely to oppose managed retreat of landward boundaries (Scholte et al. 2016).

Global warming creates other pressures on salt marshes. Increased storminess, precipitation and drought will impact saltmarsh properties, community composition and functioning (Gedan et al. 2009; Gabler et al. 2017). It is possible that livestock impacts will interact with these other drivers. For example, the increased soil salinity with grazing (Chapter 2) may exacerbate the negative effects of drought. As temperatures increase, salt marshes at lower latitudes will be replaced by mangroves (Gabler et al. 2017). Mangroves are functionally similar for some ES, including carbon 
sequestration and storage (Chmura et al. 2003; Pidgeon 2009), but offer reduced coastal protection (Narayan et al. 2016), and are not useful as grazing land.

Conversion of mangrove to cattle pasture results in huge reductions in carbon stocks (Kauffman et al. 2015), whereas salt marshes can be used for grazing with less severe (US) or no (Europe) reductions in carbon stock (Chapters 2 and 3).

In this thesis I have reported significant impacts of livestock on multiple saltmarsh properties. However, there are no reports of a complete loss of saltmarsh vegetation with livestock grazing, such as can occur with stress from droughts (McKee et al. 2004; Silliman et al. 2005), runaway consumer control caused by overharvesting of predators (reviewed by Gedan et al. 2009), hydrologic alteration and conversion of land (reviewed by Gedan et al. 2009), or natural dynamic processes (Ladd et al. in press). Graziers have an invested interest in ensuring that their pastures are not overexploited to the point of ecosystem collapse, and livestock grazing does not appear to pose such a serious risk to salt marshes and their services as other anthropogenic threats. However, questions remain as to whether livestock grazing may interact with, and exacerbate, other threats to saltmarsh functioning and resilience.

\subsection{Reflections on the ecosystem services approach}

Ecologists are increasingly aligning their research with the ES framework, but RootBernstein and Jaksic (2017) argue that this is rarely appropriate, artificially restricts questions and methodology, and obscures the interpretation of ecological data. For example, due to the ES focus of this thesis, and the intention to study anthropogenic impacts, I have considered only domesticated, or formerly domesticated, livestock as drivers. I have considered other grazers (e.g. geese, crabs, snails) as responders only and have not controlled for or considered them as drivers. However, these smaller wild herbivores may have similar, and significant, impacts on saltmarsh properties and functioning (He and Silliman 2016). The relative importance of these grazers relative to livestock, and potential synergistic effects (e.g. by livestock grazing facilitating other forms of grazing), will not be revealed by an ES-focussed approach such as this. 
The ES paradigm brings other challenges for the ecologist. ES are commonly categorised into provisioning, regulating, cultural, and, depending on the framework, supporting (or habitat) services (MA 2005a; Mace et al. 2011; Boerema et al. 2016; Haines-Young and Potschin 2018; TEEB 2019). These categories help to communicate the broad variety of ES supplied by nature, and allow analysis of whether some types of ES (usually provisioning) are commonly trading-off against other types (e.g. Howe et al. 2014). However, these categories oversimply a complex and inter-related system. For example, activities such as wildfowling provide meat, but are primarily undertaken as recreational pursuits (Paul Leyshon, Morlais Valley Shooting Club, 5 Dec 2017, personal communication), making them more cultural than provisioning, although the balance may change from person-to-person and region-to-region. Limitation of ES to a single category (in order to prevent double-counting, for example) underplays the myriad benefits that can accrue from a single ES. Cultural services, in particular, commonly overlap and interact with other ES categories, prompting suggestions to rethink the current approach and introducing new terminologies and frameworks such as 'relational values' (Chan et al. 2016), 'nonmaterial services' (Small et al. 2017) and 'nature's contributions to people' (Ellis et al. 2019). There is also the question of how to incorporate biodiversity within the ES framework, as I described in the thesis Introduction (Chapter 1). It has been my experience that pursuing an ES approach, and the consequent negotiation of multiple frameworks, critiques, and conflicting and newly arising terminologies, can lead to the expenditure of much intellectual effort considering arbitrary and often unnecessary questions of semantics.

Quantifying ES adequately is particularly challenging. The limitations of economic valuation methods have been covered elsewhere (Silvertown 2015; Root-Bernstein and Jaksic 2017) and I have demonstrated high variability in the provision of individual ES from salt marshes, calling into question the accuracy of land use/land cover as a proxy for ES supply. In this thesis I have used various approaches to measure or value ES, falling along multiple points of the ES cascade (Fig. 1.1). I have variously measured properties (e.g. biomass, soil organic matter), functions (e.g. decomposition), potential ES supply (e.g. potential birdwatching value), and realised ES supply (number of wildfowl shot). These measures were selected based on the 
availability of data or the ease of collecting primary data, and may not be the most accurate or appropriate measures. For example, I classified wildfowling as a recreational ES, so the number of wildfowl shot may not be the best measure of this service - the number of man hours spent on the marsh, or the number of active club members may be more appropriate measures. Due to limited time and resources, ES assessments will always be biased towards services that are easily quantified and have readily-available data, meaning that some ES are likely to be over-emphasised while others with less tangible benefits are neglected (Small et al. 2017).

Additionally, relating each ecosystem property or function to an ES requires one to make assumptions about how these aspects are related, based on relatively limited understanding of the underlying ecological and socio-cultural mechanisms. Each property or function is likely to feed into multiple ES, and each ES may rely on multiple properties or functions (Duncan et al. 2015). Furthermore, the relationships between elements on the ES cascade may not be linear - there may be thresholds of properties or functions under which no service is delivered (e.g. below a particular density of flower cover, it may be inefficient for bees to visit a habitat patch), or thresholds of services under which no benefit is delivered (e.g. below a particular grazing density, it may be uneconomical for a farmer to keep stock at all). For a true assessment of ES trade-offs and ES multifunctionality, the property-function-supplybenefit relationship, and relevant thresholds, must be derived for every ES (Manning et al. 2018). My results have demonstrated that impacts of grazing are clearly evident when properties are measured (Chapter 2), but impacts on functions and services are harder to detect (Chapters 3 and 5).

In this thesis I have not undertaken any stakeholder engagement to assess the demand for particular ES. Stakeholder engagement is strongly recommended to gain a complete understanding of ES provision, accounting for both supply and demand (Bennett 2016; Manning et al. 2018). However, it is rarely used in practice (Boerema et al. 2016), probably because it is time-consuming and requires inter-disciplinary expertise, incorporating social science methods into natural science research. When it is undertaken, stakeholder engagement allows services to be weighted according to their value for different groups, allowing more meaningful ES-multifunctionality measures to be derived (Manning et al. 2018) and recognition of trade-offs and 
power imbalances between different groups (Howe et al. 2014; Cavender-bares et al. 2015). However, while stakeholder weightings are informative, they cannot be the only means of prioritising ES, as this risks prioritisation of tangible or visible ES over intangible or invisible ES, charismatic species over uncharismatic species, local benefits over global benefits, and immediate benefits over sustainable supply. For these reasons, stakeholder weightings must be used in conjunction with strong scientific guidance (Rassweiler et al. 2014). A key value of stakeholder engagement is in promoting a two-way flow of information and knowledge between researchers, policy-makers and stakeholders (Rassweiler et al. 2014; Beaumont et al. 2017; McKinley et al. 2019). This engagement can reduce conflicts, and produce integrated management plants with broad stakeholder support (Arkema et al. 2015).

As described above, the ES approach imposes many restrictions and challenges when used for ecological research, and may not always be the best approach when addressing ecological questions. However, it does force a consideration of the wider social, cultural and economic forces that both react to, and impact on, the natural world. Despite criticisms of it encouraging a human-centric view of nature (Monbiot 2014), a real success of the ES concept has been in communicating the huge benefits that all people gain from nature, and in engaging people who do not, and perhaps never will, value nature for its own sake. Nature and biodiversity are now recognised by world governments as key forces promoting human wellbeing (e.g. in the United Nations Sustainable Development Goals and the Well-being of Future Generations (Wales) Act 2015). The ES approach can therefore be a very useful tool in engaging policy makers and the public in environmental matters. However, the ES approach should be used as one tool among many when determining optimal environmental management, as human interests - specifically, easily defined and quantified human interests - cannot be the only force driving our management of the natural world.

\subsection{Future research questions}

This thesis has highlighted many evidence gaps, and a better understanding of grazing impacts, saltmarsh ES, and ES in general, would be reached by addressing the following key research questions. 


\section{Ecological questions}

1. What are the linkages between ecosystem properties, ecosystem functions (EFs) and ES in US and UK salt marshes? What properties and EFs (or bundles of EFs) are required, and at what levels, to provide each ES? A better understanding of these links would facilitate better predictions of how alterations to saltmarsh properties, including via grazing management, will impact ES supply.

2. What are the consequences of the observed grazer-driven changes to plant community composition for ES provisioning in US and UK salt marshes? This requires an assessment of functional traits-EF relationships, rather than simply biodiversityEF relationships.

3. What are the effects of saltmarsh grazing on the marine ecosystem, particularly aquatic food-webs and coastal water quality?

4. What are the effects of saltmarsh grazing on wave attenuation and the provision of coastal protection from storms?

5. How does livestock grazing interact with other stressors, such as sea-level rise, drought, and herbivory from wild animals? How will this impact on the long-term stability of salt marshes and their ES supply?

6. How does ES provision vary within (rather than between) other land use/land cover (LULC) types? What is the range of variation in ES supply within different LULC classes compared to the variation between classes, and are there any consistent factors that predict changing provision within a LULC? This knowledge would facilitate mapping and modelling of ES across wider landscapes, and provide a deeper understanding of the mechanisms driving trade-offs and synergies.

\section{Sociological questions}

1. What is the demand for various saltmarsh ES from stakeholders, and do current levels of supply meet demand? Do different groups of stakeholders have differing priorities, and do all groups of stakeholders have a voice in shaping management priorities and policies? 
2. Here I have considered grazing exclusively as a provisioning service. To what extent does grazing also provide cultural services e.g. heritage value for successive generations of farmers, contribution to 'sense-of-place', aesthetic appreciation of an agricultural landscape (UK) or free-roaming horses (US)? 


\section{Appendix A: Supporting information for Chapter 2}

\section{Contents}

Supplementary methods. Study selection and data extraction.

Table A1. Ecosystem properties investigated and list of data sources.

Table A2. Full results of weighted meta-analysis.

Table A3. Full results of regression analyses.

Fig. A1. Forest plots and funnel plots for all EPs.

Fig. A2. Results of coded meta-analysis.

Fig. A3. Predicted moderating effects of stocking density, duration and identity of grazer (significant at $\mathrm{P}<0.05$ ).

Fig. A4. Predicted moderating effects of dominant vegetation in Europe 145 (significant at $\mathrm{P}<0.05$ ). 


\section{Supplementary methods - Study selection and data extraction}

Study selection

Keyword searches in Scopus and Web of Science were used to identify peer-reviewed literature quantifying the effects of livestock grazing on the EPs of salt marshes. We retrieved papers published from 1950 to November 2015 containing the terms (saltmarsh* OR "salt marsh*" OR salt-marsh OR "salt meadow" OR "coastal marsh") AND (graz* OR herbivor* OR livestock OR cow* OR cattle OR sheep OR horse* OR deer) in their title, abstract or keywords. Additionally, the doctoral thesis repository EThOS was searched in November 2015 for PhD theses containing these terms.

Only papers published in English, or with a comprehensive English summary, were included. Properties were not fixed a priori, to allow the available data to dictate what information was extracted. We broadly searched for ecosystem properties (EPs) relating to the abiotic (accretion, sedimentation, soil properties, water quality) and biotic (vegetation properties, any measure of species richness or abundance) environment that may affect ecosystem functioning and service provision. The search yielded 1300 records from Scopus and Web of Science and 15 theses from EThOS. The title and abstract of each record was examined to determine if they met the inclusion criteria. Where this could not be determined from the title and abstract, the paper was retained until the next stage of analysis. Following filtration, 290 references remained which we attempted to retrieve. Where papers were not available through institutional subscriptions or inter-library loans, the corresponding author was contacted by email. Of these filtered results, 201 were excluded; 150 did not meet the inclusion criteria, 26 were published in a foreign language and 25 could not be obtained.

The inclusion of grey literature is recommended for systematic reviews, to minimise publication bias (Pullin and Stewart 2006; Koricheva et al. 2013). Due to limited resources, we were not able to include it here. Many properties assessed in this review were reported as contextual environmental data, rather than being the primary outcome for their respective studies, so are less likely to be subject to reporting bias.

\section{Data extraction}

We extracted grazing outcomes (entries) from the figures, tables and text of each study. Where the authors investigated EPs at several grazing intensities, data from all intensities were included as separate entries, to allow stocking density to be 
investigated as a potential moderator of the grazing effect. Where responses from different marsh zones (i.e. pioneer, low, middle, upper) were presented separately, and where biotic data were presented separately per functional group (e.g. herbivorous invertebrates), these were added as separate entries. Where data were collected from multiple depths belowground, and no average value was provided, the data from the uppermost layer of soil was included, as this is closest to the activity of the grazing livestock. Where EPs were measured over an extended time period, and no overall average was provided, only the final measurement was extracted. However, when results were presented separately by season, and no year average was provided, an overall average and SD for the year was calculated using successive iterations of Equation S1 via the StatsToDo computer program (StatsToDo 2016).

$$
S D_{1,2}=\sqrt{\frac{N_{1} S D_{1}^{2}+N_{2} S D_{2}^{2}+N_{1}\left(\bar{X}_{1}-\bar{X}_{12}\right)^{2}+N_{2}\left(\bar{X}_{2}-\bar{X}_{12}\right)^{2}}{N_{1}+N_{2}}}
$$

Where $S D=$ standard deviation, $N=$ sample size, $\bar{X}=$ mean, 1 = first dataset, 2 = second dataset and 1,2 = combined datasets.

In total, 498 entries for 26 properties were extracted from the 89 included studies (Table A1). There were insufficient entries for accretion rate, sedimentation rate and vertical elevation change to analyse each separately, so these were grouped together under the EP of 'accretion'. The values for soil carbon included measures of carbon concentration, carbon density and soil organic matter. Values for redox potential can be negative or positive, making the raw data unsuitable for conversion to an effect size using ratios. We therefore rescaled this variable above 0 by adding 300 to each mean value (-300 $\mathrm{mV}$ is the minimum redox potential in wetland soils, DeLaune and Reddy 2005) before calculating the log response ratio ( $\ln R R)$.

Where possible, study-specific variables were recorded for each entry. Study location was noted by site name and country. Moderators related to grazing management were recorded: the species of the grazer, the time (in years) since introduction/exclusion of grazing and the stocking density (most often reported as animals per hectare). Where plots were described as being grazed 'long term', this was estimated at 100 years. To allow a comparison of stocking density for different species, the reported stocking rate was converted into a common metric of livestock units per hectare (LSU/ha) using conversion factors ( 1 cow or horse $=1,1$ pony $=0.5$, 1 deer $=0.3,1$ sheep or goat $=0.12$ LSU) based on UK government classifications (Welsh Government 2013b; Scottish Government 2016). The dominant plant species in ungrazed and grazed areas were noted and classified as Spartina, $\mathrm{C}_{3}$ graminoids, or forbs (using Bruhl and Wilson 2007; Atia et al. 2014; Osborne et al. 2014). No plots 
were dominated by shrubs. Marsh zone was recorded as pioneer, low, middle or high. Soil type was recorded as sand or clay. The study type was classified as: artificial replication of grazing by clipping or trampling, grazing exclosure, grazing enclosure, laboratory study, observation of existing grazed and ungrazed areas, or paired plots on either side of a boundary fence. Study type was not tested as a potential moderator, as it was strongly correlated with grazing duration: experimental results were recorded after a significantly shorter duration of grazing (median $=4 \mathrm{y}$ ) than observational results (median=30 y; Mann-Whitney $U$ test: $W=2037.5, p<0.0001$ ). 
Table A1. Ecosystem properties investigated and list of data sources. CMA = coded meta-analysis, WMA = weighted meta-analysis, UMR=unweighted meta-regression. Sources shown in bold were used only in the weighted meta-analysis and coded meta-analysis, sources in italics were used only in the unweighted meta-regression and coded meta-analysis, sources in square brackets were used only in the coded meta-analysis.

\begin{tabular}{|c|c|c|c|c|}
\hline \multirow[t]{2}{*}{ Ecosystem property } & \multicolumn{3}{|c|}{ Entries (studies) } & \multirow[t]{2}{*}{ Sources } \\
\hline & CMA & WMA & UMR & \\
\hline $\begin{array}{l}\text { Accretion } \\
\text { (Inc. elevation change, } \\
\text { sedimentation) }\end{array}$ & $13(6)$ & $5(3)$ & & {$[1], 25,[54], 56,71,[82]$} \\
\hline Bulk density & $27(16)$ & $21(13)$ & $21(13)$ & $\begin{array}{l}{[5], 20,25,28,[33], 40,50,52,56,} \\
57,59,63,65,69,[78], 89\end{array}$ \\
\hline Creek stability & $1(1)$ & & & {$[32]$} \\
\hline $\begin{array}{l}\text { Redox } \\
\text { (Values adjusted by }+300 \text { to } \\
\text { rescale above } 0 \text { ) }\end{array}$ & $12(6)$ & $12(6)$ & $12(6)$ & $18,24,33,65,78,85$ \\
\hline $\mathrm{pH}$ & 9(7) & $8(6)$ & & $20,[23], 27,28,57,63,89$ \\
\hline Salinity & 23(12) & $17(9)$ & $17(9)$ & $\begin{array}{l}{[6], 19,21,26,28,33,50,58,[78],} \\
{[82], 85,89}\end{array}$ \\
\hline $\begin{array}{l}\text { Soil carbon } \\
\text { (Inc. soil carbon density, } \\
\text { concentration \& organic matter) }\end{array}$ & 29(18) & $23(14)$ & $27(16)$ & $\begin{array}{l}{[5], 20,[23], 24,27,28,40,41,50,} \\
52,56,57,59,63,65,69,85,89\end{array}$ \\
\hline Soil strength & $4(2)$ & & & {$[41],[64]$} \\
\hline Surface heterogeneity & $4(1)$ & & & [8] \\
\hline Daytime temperature & $6(5)$ & $5(4)$ & & $28,57,58,85,[89]$ \\
\hline Aboveground biomass & $36(20)$ & $26(15)$ & $35(19)$ & $\begin{array}{l}5,9,19,22,24,28,30,31,[35], 40 \\
41,51,52,57,59,60,74,77,86,89\end{array}$ \\
\hline Belowground biomass & $17(12)$ & $14(11)$ & $17(12)$ & $\begin{array}{l}22,24,28,30,31,40,41,52,57,59 \\
64,89\end{array}$ \\
\hline Canopy height & $42(23)$ & $22(14)$ & $54(19)$ & $\begin{array}{l}1,[2], 8,[10], 12,14,16,25,28,30 \\
33,[35], 39,41,49,58,61,63,67 \\
{[69], 76,79,87}\end{array}$ \\
\hline Flower production & $8(2)$ & & & {$[9],[55]$} \\
\hline Litter biomass & $17(9)$ & $11(6)$ & 17(9) & $5,19,24,28,52,60,61,74,89$ \\
\hline Nitrogen content & $11(6)$ & $4(3)$ & $11(6)$ & $12,51,60,63,76,77$ \\
\hline $\begin{array}{l}\text { Percentage cover } \\
\text { (Or } 100 \% \text { - bare ground) }\end{array}$ & 28(17) & $14(10)$ & 23(15) & $\begin{array}{l}{[1], 4,6,21,22,31,32,[34],[35], 41,} \\
49,51,58,61,63,75,84,87\end{array}$ \\
\hline Primary production & $5(3)$ & & & {$[28],[52],[74]$} \\
\hline $\begin{array}{l}\text { Reproduction } \\
\text { (Includes number, emergence, } \\
\text { survival of seedlings) }\end{array}$ & $3(3)$ & & & [6], [9], [37] \\
\hline Species richness & $64(34)$ & $30(17)$ & $54(26)$ & $\begin{array}{l}{[5], 6,7,8,9,[10], 11,15,21,[23],} \\
{[26], 29,31,[34], 36,37,38,41,[42],} \\
49,51,57,60,61,[62], 63,66,72,75, \\
79,[80], 81,83,84\end{array}$ \\
\hline Stem density & $6(3)$ & & & {$[10],[14],[76]$} \\
\hline Invertebrate abundance & $56(19)$ & $37(13)$ & $42(15)$ & $\begin{array}{l}{[1], 2,3,[10], 19,22,31,33,46,50,} \\
59,60,61,[65], 69,73,79,[78], 85\end{array}$ \\
\hline Invertebrate richness & 19(10) & 16(9) & 19(10) & $1,2,29,46,58,59,61,73,79,85$ \\
\hline Vertebrate abundance & $31(15)$ & $13(8)$ & 26(12) & $\begin{array}{l}{[10], 12,13,14,16,17,43,[44],[45],} \\
47,48,67,69,[70], 76,77\end{array}$ \\
\hline Vertebrate richness & $4(2)$ & & & {$[17],[47]$} \\
\hline Wader nest survival & $3(3)$ & & & {$[10],[68],[88]$} \\
\hline
\end{tabular}


1. Andresen, H., Bakker, J. P., Brongers, M., Heydemann, B. \& Irmler, U. (1990) Long-term changes of salt marsh communities by cattle grazing. Vegetatio, 89, 137-148.

2. Armonies, W. (1986). Free-living plathelminthes in sheep-grazed and ungrazed supralittoral salt marshes of the North Sea: Abundance, biomass, and their significance in food chains. Netherlands Journal of Sea Research, 20(4), 385-395. http://doi.org/10.1016/00777579(86)90005-0

3. Aydin, G (2011). Vulnerability of Megacephala (Grammognatha) euphratica euphratica Latreille \& Dejean, 1822 (Coleoptera: Cicindelidae) in natural and disturbed saltmarsh and salt meadow habitats in Turkey. African Journal of Biotechnology, 10, 5692-5696.

4. Bakker, J. P. \& Ruyter, J. C. (1981) Effects of five years of grazing on a salt-marsh vegetation A study with sequential mapping. Vegetatio, 44, 81-100.

5. Bakker, J. P. (1985) The impact of grazing on plant communities, plant populations and soil conditions on salt marshes. Vegetatio, 62, 391-398.

6. Bakker, J. P., Dijkstra, M. \& Russchen, P. T. (1985). Dispersal, germination and early establishment of halophytes and glycophytes on a grazed and abandoned salt-marsh gradient. New Phytologist, 101, 291-308.

7. Bakker, J. P., Bos, D. \& De Vries, Y. (2003) To graze or not to graze: that is the question. In K. Essink, M. van Leeuwe, A. Kellermann \& W. Wolff (Eds.), Proceedings of the 10th International Scientific Wadden Sea Symposium (pp. 67-88). Ministry of Agriculture, Nature management and Fisheries, The Hague, and Department of Marine Biology, University of Groningen.

8. Berg, G., Esselink, P., Groeneweg, M. \& Kiehl, K. (1997) Micropatterns in Festuca rubradominated salt-marsh vegetation induced by sheep grazing. Plant Ecology, 132, 1-14.

9. Blom, C. W. P. M. (1983) Plasticity of life characteristics in two different populations of Plantago maritima L. Acta Oecologica, 4, 377-394.

10. Böhme, B., Främbs, H., Hielen, B. \& Kinder, M. (1999) Salt marsh project "Wurster Kuste": Regeneration of coastal grasslands and salt marshes. Senckenbergiana Maritima, 29(Suppl.), 37-42.

11. Bos, D., Bakker, J. P., de Vries, Y. \& van Lieshout, S. (2002) Long-term vegetation changes in experimentally grazed and ungrazed back-barrier marshes in the Wadden Sea. Applied Vegetation Science, 5, 45-54.

12. Bos, D., Drent, R. H., Rubinigg, M. \& Stahl, J. (2005). The relative importance of food biomass and quality for patch and habitat choice in Brent Geese Branta bernicla. Ardea, 93, 5-16.

13. Bos, D., Boersma, S., Engelmoer, M., Veeneklaas, R. M., Bakker, J. P. \& Esselink, P. (2014). Utilisation of a coastal grassland by geese after managed re-alignment. Journal of Coastal Conservation, 18, 471-479.

14. Bos, D., Loonen, M. J. J. E., Stock, M., Hofeditz, F., van der Graaf, A. J. \& Bakker, J. P. (2005) Utilisation of Wadden Sea salt marshes by geese in relation to livestock grazing. Journal for Nature Conservation, 13, 1-15.

15. Bouchard, V., Tessier, M., Digaire, F., Vivier, J. P., Valery, L., Gloaguen, J. C. \& Lefeuvre, J. C. (2003) Sheep grazing as management tool in Western European saltmarshes. Comptes Rendus - Biologies, 326(Suppl.), S148-S157.

16. Cardoni, D. A., Isacch, J. P. \& Iribarne, O. (2012) Effects of cattle grazing and fire on the abundance, habitat selection, and nesting success of the Bay-capped Wren-Spinetail (Spartonoica maluroides) in coastal saltmarshes of the Pampas region. The Condor, 114, 803811.

17. Cardoni, D. A., Isacch, J. P. \& Iribarne, O. (2015) Avian responses to varying intensity of cattle production in Spartina densiflora saltmarshes of south-eastern South America. Emu, 115, 1219. 
18. Chang, E. R., Veeneklaas, R. M., Bakker, J. P., Daniels, P. \& Esselink, P. (2015) What factors determined restoration success of a salt marsh ten years after de-embankment? Applied Vegetation Science, doi:10.1111/avsc.12195.

19. Comor, V., Thakur, M. P., Berg, M. P., de Bie, S., Prins, H. H. T. \& van Langevelde, F. (2014) Productivity affects the density-body mass relationship of soil fauna communities. Soil Biology and Biochemistry, 72, 203-211.

20. Di Bella, C. E., Rodríguez, A. M., Jacobo, E., Golluscio, R. A. \& Taboada, M. A. (2015) Impact of cattle grazing on temperate coastal salt marsh soils. Soil Use and Management, 31, 299-307.

21. Di Bella, C. E., Jacobo, E., Golluscio, R. A. \& Rodriguez, A. M. (2014). Effect of cattle grazing on soil salinity and vegetation composition along an elevation gradient in a temperate coastal salt marsh of Samborombon Bay (Argentina). Wetlands Ecology and Management, 22, 1-13.

22. Diaz, A., Pinn, E. \& Hannaford, J. (2005) Ecological impacts of Sika Deer on Poole Harbour saltmarshes. In V. May \& J. Humphreys (Eds.), The Ecology of Poole Harbour.(pp. 175-188). Elsevier Science \& Technology, Amsterdam.

23. Dupre, C. \& Diekmann, M. (2001) Differences in species richness and life-history traits between grazed and abandoned grasslands in southern Sweden. Ecography, 24, 275-286.

24. Elschot, K., Bakker, J., Temmerman, S., van de Koppel, J. \& Bouma, T. (2015) Ecosystem engineering by large grazers enhances carbon stocks in a tidal salt marsh. Marine Ecology Progress Series, 537, 9-21.

25. Elschot, K., Bouma, T. J., Temmerman, S. \& Bakker, J. P. (2013) Effects of long-term grazing on sediment deposition and salt-marsh accretion rates. Estuarine, Coastal and Shelf Science, 133, 109-115.

26. Esselink, P., Fresco, L. F. M. \& Dijkema, K. S. (2002). Vegetation change in a man-made salt marsh affected by a reduction in both grazing and drainage. Applied Vegetation Science, $\mathbf{5}$, 17-32.

27. Ford, H., Rousk, J., Garbutt, A., Jones, L. \& Jones, D. L. (2013) Grazing effects on microbial community composition, growth and nutrient cycling in salt marsh and sand dune grasslands. Biology and Fertility of Soils, 49, 89-98.

28. Ford, H., Garbutt, A., Jones, L. \& Jones, D. L. (2012) Methane, carbon dioxide and nitrous oxide fluxes from a temperate salt marsh: Grazing management does not alter Global Warming Potential. Estuarine, Coastal and Shelf Science, 113, 182-191.

29. Ford, H., Garbutt, A., Jones, L. \& Jones, D. L. (2013) Grazing management in saltmarsh ecosystems drives invertebrate diversity, abundance and functional group structure. Insect Conservation and Diversity, 6, 189-200.

30. Gouraud, C., Giroux, J.-F., Mesleard, F. \& Desnouhes, L. (2008) Non-destructive sampling of Schoenoplectus maritimus in southern France. Wetlands, 28, 532-537.

31. Hannaford, J., Pinn, E. H. \& Diaz, A. (2006). The impact of sika deer grazing on the vegetation and infauna of Arne saltmarsh. Marine Pollution Bulletin, 53, 56-62.

32. House, C., May, V. \& Diaz, A. (2005). Sika Deer trampling and saltmarsh creek erosion: Preliminary investigation. In J. Humphreys \& V. May (Eds.), The Ecology of Poole Harbour (pp. 189-193). Elsevier Science \& Technology, Amsterdam.

33. Howison, R. A., Olff, H., Steever, R. \& Smit, C. (2015) Large herbivores change the direction of interactions within plant communities along a salt marsh stress gradient. Journal of Vegetation Science, 26, 1159-1170.

34. Jensen, A. (1985) The effect of cattle and sheep grazing on salt-marsh vegetation at Skallingen, Denmark. Vegetatio, 60, 37-48.

35. Jutila, H. (1999) Effect of grazing on the vegetation of shore meadows along the Bothnian Sea, Finland. Plant Ecology, 140, 77-88.

36. Jutila, H. M. (1998) Effect of different treatments on the seed bank of grazed and ungrazed Baltic seashore meadows. Canadian Journal of Botany, 76. 
37. Jutila, H. M. (1998) Seed banks of grazed and ungrazed Baltic seashore meadows. Journal of Vegetation Science, 9, 395-408.

38. Jutila, H. M. (1997) Vascular plant species richness in grazed and ungrazed coastal meadows, SW Finland. Annales Botanici Fennici, 34, 245-263.

39. Kiehl, K., Eischeid, I., Gettner, S. \& Walter, J. (1996) Impact of different sheep grazing intensities on salt marsh vegetation in northern Germany. Journal of Vegetation Science, $\mathbf{7}$, 99-106.

40. Kiehl, K., Esselink, P., Gettner, S. \& Bakker, J. P. (2001) The impact of sheep grazing on net nitrogen mineralization rate in two temperate salt marshes. Plant Biology, 3, 553-560.

41. Kingham, R. (2013) The broad-scale impacts of livestock grazing on saltmarsh carbon stocks. PhD thesis, Bangor University.

42. Kleyer, M., Feddersen, H. \& Bockholt, R. (2003) Secondary succession on a high salt marsh at different grazing intensities. Journal of Coastal Conservation, 9, 123-134.

43. Kuijper, D. P. J., Beek, P., van Wieren, S. E. \& Bakker, J. P. (2008). Time-scale effects in the interaction between a large and a small herbivore. Basic and Applied Ecology, 9, 126-134.

44. Laffaille, P., Lefeuvre, J. \& Feunteun, E. (2000) Impact of sheep grazing on juvenile sea bass, Dicentrarchus labrax L., in tidal salt marshes. Biological Conservation, 96, 271-277.

45. Laursen, K. (1977) The effect of reed harvesting on a population of passerine birds. Dansk Ornitologisk Forenings Tidsskrift, 71, 95-101.

46. Leroy, B., Le Viol, I. \& Pétillon, J. (2014) Complementarity of rarity, specialisation and functional diversity metrics to assess community responses to environmental changes, using an example of spider communities in salt marshes. Ecological Indicators, 46, 351-357.

47. Levin, P. S., Ellis, J., Petrik, R. \& Hay, M. E. (2002) Indirect effects of feral horses on estuarine communities. Conservation Biology, 16, 1364-1371.

48. Malpas, L. R., Smart, J., Drewitt, A., Sharps, E. \& Garbutt, A. (2013) Continued declines of Redshank Tringa totanus breeding on saltmarsh in Great Britain: is there a solution to this conservation problem? Bird Study, 60, 370-383.

49. Meirland, A., Bouvet, A., Rybarczyk, H., Dubois, F. \& Chabrerie, O. (2013) Effects of sheep grazing on salt-marsh plant communities in the bay of Somme (France). Revue d'Ecologie (La Terre et La Vie), 68, 319-333.

50. Meyer, H., Fock, H., Haase, A., Reinke, H. D. \& Tulowitzki, I. (1995) Structure of the invertebrate fauna in salt marshes of the Wadden Sea coast of Schleswig-Holstein influenced by sheep-grazing. Helgoländer Meeresuntersuchungen, 49, 563-589.

51. Milotic, T., Erfanzadeh, R., Petillon, J., Maelfait, J. P. \& Hoffmann, M. (2010). Short-term impact of grazing by sheep on vegetation dynamics in a newly created salt-marsh site. Grass and Forage Science, 65, 121-132.

52. Morris, J. T. \& Jensen, A. (1998). The carbon balance of grazed and non-grazed Spartina anglica saltmarshes at Skallingen, Denmark. Journal of Ecology, 86, 229-242.

53. Müller, F., Struyf, E., Hartmann, J., Weiss, A. \& Jensen, K. (2013). Impact of grazing management on silica export dynamics of Wadden Sea saltmarshes. Estuarine, Coastal and Shelf Science, 127, 1-11.

54. Neuhaus, R., Stelter, T. \& Kiehl, K. (1999). Sedimentation in salt marshes affected by grazing regime, topographical patterns and regional differences. Senckenbergiana Maritima, 29(Suppl.), 113-116.

55. Nolte, S., Esselink, P. \& Bakker, J. P. (2013). Flower production of Aster tripolium is affected by behavioral differences in livestock species and stocking densities: the role of activity and selectivity. Ecological Research, 28, 821-831.

56. Nolte, S., Mueller, F., Schuerch, M., Wanner, A., Esselink, P., Bakker, J. P. \& Jensen, K. (2013) Does livestock grazing affect sediment deposition and accretion rates in salt marshes? Estuarine Coastal and Shelf Science, 135, 296-305. 
57. Olsen, Y. S., Dausse, A., Garbutt, A., Ford, H., Thomas, D. N. \& Jones, D. L. (2011). Cattle grazing drives nitrogen and carbon cycling in a temperate salt marsh. Soil Biology and Biochemistry, 43, 531-541.

58. Petillon, J., Georges, A., Canard, A. \& Ysnel, F. (2007) Impact of cutting and sheep grazing on ground-active spiders and carabids in intertidal salt marshes (Western France). Animal Biodiversity and Conservation, 30, 201-209.

59. Reader, J. \& Craft, C. (1999) Comparison of wetland structure and function on grazed and ungrazed salt marshes. Journal of the Elisha Mitchell Scientific Society, 115, 236-249.

60. Reimold, R. J., Linthurst, R. A. \& Wolf, P. L. (1975) Effects of grazing on a salt marsh. Biological Conservation, 8, 105-125.

61. Rickert, C., Fichtner, A., van Klink, R. \& Bakker, J. P. (2012) Alpha- and beta-diversity in moth communities in salt marshes is driven by grazing management. Biological Conservation, 146, 24-31.

62. Ruifrok, J. L., Postma, F., Olff, H. \& Smit, C. (2014) Scale-dependent effects of grazing and topographic heterogeneity on plant species richness in a Dutch salt marsh ecosystem. Applied Vegetation Science, 17, 615-624.

63. Sammul, M., Kauer, K. \& Köster, T. (2012) Biomass accumulation during reed encroachment reduces efficiency of restoration of Baltic coastal grasslands. Applied Vegetation Science, 15, 219-230.

64. Scholand, M., Austenfeld, F. A. \& Von Willert, D. J. (1991). Underground biomass and its influence on soil shear strength in a grazed and an ungrazed German coastal marsh. In Atkinson, D. (Ed.) Plant Root Growth (pp. 341-348). Blackwell Scientific, Oxford.

65. Schrama, M., Heijning, P., Bakker, J. P., van Wijnen, H. J., Berg, M. P. \& Olff, H. (2013) Herbivore trampling as an alternative pathway for explaining differences in nitrogen mineralization in moist grasslands. Oecologia, 172, 231-243.

66. Schroder, H. K., Kiehl, K. \& Stock, M. (2002) Directional and non-directional vegetation changes in a temperate salt marsh in relation to biotic and abiotic factors. Applied Vegetation Science, 5, 33-44.

67. Sharps, E. A. (2015) The effects of saltmarsh conservation grazing on breeding Common Redshank Tringa totanus. PhD thesis, Bangor University.

68. Sharps, E., Smart, J., Skov, M. W., Garbutt, A. \& Hiddink, J. A. N. G. (2015) Light grazing of saltmarshes is a direct and indirect cause of nest failure in Common Redshank Tringa totanus. Ibis, 157, 239-249.

69. Smith, D. J. (2013) Changes in perspectives of the values and benefits of nature. PhD thesis, University of Salford.

70. Stock, M. \& Hofeditz, F. (2002) Impact of salt marsh management on habitat utilisation and population development of Barnacle Geese Branta leucopsis in the Wadden Sea. Vogelwelt, 123, 265-282.

71. Suchrow, S., Pohlmann, N., Stock, M. \& Jensen, K. (2012). Long-term surface elevation changes in German North Sea salt marshes. Estuarine, Coastal and Shelf Science, 98, 71-83.

72. Suchrow, S., Stock, M. \& Jensen, K. (2015) Patterns of plant species richness along environmental gradients in German North Sea salt marshes. Estuaries and Coasts, 38, 296309.

73. Thakur, M. P., Berg, M. P., Eisenhauer, N. \& van Langevelde, F. (2014) Disturbance-diversity relationships for soil fauna are explained by faunal community biomass in a salt marsh. Soil Biology and Biochemistry, 78, 30-37.

74. Turner, M. G. (1987) Effects of grazing by feral horses, clipping, trampling, and burning on a Georgia Salt Marsh. Estuaries, 10, 54-60. 
75. Ungar, I. A. \& Woodell, S. R. J. (1996) Similarity of seed banks to aboveground vegetation in grazed and ungrazed salt marsh communities on the Gower Peninsula, South Wales. International Journal of Plant Sciences, 157, 746.

76. van der Graaf, A. J., Bos, D., Loonen, M. J. J. E., Engelmoer, M. \& Drent, R. H. (2002) Shortterm and long-term facilitation of goose grazing by livestock in the Dutch Wadden Sea area. Journal of Coastal Conservation, 8, 179-188.

77. van der Graaf, A. J., Stahl, J., Veen, G. F., Havinga, R. M. \& Drent, R. H. (2007) Patch choice of avian herbivores along a migration trajectory-From Temperate to Arctic. Basic and Applied Ecology, 8, 354-363.

78. van Klink, R., Schrama, M., Nolte, S., Bakker, J. P., WallisDeVries, M. F. \& Berg, M. P. (2015) Defoliation and soil compaction jointly drive large-herbivore grazing effects on plants and soil arthropods on clay soil. Ecosystems, 18, 671-685.

79. van Klink, R., Rickert, C., Vermeulen, R., Vorst, O., WallisDeVries, M. F. \& Bakker, J. P. (2013) Grazed vegetation mosaics do not maximize arthropod diversity: Evidence from salt marshes. Biological Conservation, 164, 150-157.

80. Van Wijnen, H. J., Bakker, J. P. \& De Vries, Y. (1997) Twenty years of salt marsh succession on a Dutch coastal barrier island. Journal of Coastal Conservation, 3, 9-18.

81. Veeneklaas, R., Bockelmann, A., Reusch, T. \& Bakker, J. (2011) Effect of grazing and mowing on the clonal structure of Elytrigia atherica: A long-term study of abandoned and managed sites. Preslia, 83, 455-470.

82. Veenklaas, R. M., Koppenaal, E. C., Bakker, J. P. \& Esselink, P. (2015) Salinization during saltmarsh restoration after managed realignment. Journal of Coastal Conservation, 19, 405-415.

83. Wanner, A., Suchrow, S., Kiehl, K., Meyer, W., Pohlmann, N., Stock, M. \& Jensen, K. (2014). Scale matters: Impact of management regime on plant species richness and vegetation type diversity in Wadden Sea salt marshes. Agriculture, Ecosystems \& Environment, 182, 69-79.

84. Wasson, K. \& Woolfolk, A. (2011) Salt marsh-upland ecotones in central California: Vulnerability to invasions and anthropogenic stressors. Wetlands, 31, 389-402.

85. Whitcraft, C. R. \& Levin, L. A. (2007). Regulation of benthic algal and animal communities by salt marsh plants: Impact of shading. Ecology, 88, 904-917.

86. Wood, G. W., Mengak, M. T. \& Murphy, M. (1987). Ecological importance of feral ungulates at Shackleford Banks, North Carolina. American Midland Naturalist, 118, 236-244.

87. Yang, S. L., Li, H., Ysebaert, T., Bouma, T. J., Zhang, W. X., Wang, Y. Y., Li, P., Li, M. \& Ding, P. X. (2008) Spatial and temporal variations in sediment grain size in tidal wetlands, Yangtze Delta: On the role of physical and biotic controls. Estuarine, Coastal and Shelf Science, 77, 657-671.

88. Yates, B. J. (1982) A population biology of breeding redshanks (Tringa totanus L.). PhD thesis, Liverpool Polytechnic.

89. Yu, O. T. \& Chmura, G. L. (2010) Soil carbon may be maintained under grazing in a St Lawrence Estuary tidal marsh. Environmental Conservation, 36, 312-320. 
Table A2. Results of weighted meta-analysis of the effect of livestock grazing on Ecosystem Properties. $n(N)=$ number of entries (number of studies).

\begin{tabular}{|c|c|c|c|c|c|}
\hline Ecosystem Property & $n(N)$ & $\begin{array}{l}\text { Mean effect } \\
(\ln R R)\end{array}$ & $P$ & $\begin{array}{l}\text { Cochrane's } \\
\text { Q }\end{array}$ & $\begin{array}{l}\text { Inconsistency } \\
\text { index }\left(\mathrm{I}^{2}\right)\end{array}$ \\
\hline \multicolumn{6}{|l|}{ Soil properties } \\
\hline Accretion & $5(3)$ & -0.326 & 0.318 & 89.5 & 95.5 \\
\hline Bulk density & $21(13)$ & 0.374 & 0.018 & 257.2 & 92.2 \\
\hline Carbon & $23(14)$ & 0.014 & 0.907 & 712.5 & 96.9 \\
\hline $\mathrm{pH}$ & $8(6)$ & -0.005 & 0.919 & 59.0 & 88.1 \\
\hline Redox potential & $12(6)$ & -0.438 & 0.028 & 154.7 & 92.9 \\
\hline Salinity & $17(9)$ & 0.219 & 0.017 & 38.4 & 58.3 \\
\hline Daytime temperature & $5(4)$ & 0.122 & $<0.001$ & 34.0 & 88.2 \\
\hline \multicolumn{6}{|l|}{ Vegetation properties } \\
\hline AGB & $26(15)$ & -1.113 & $<0.0001$ & 2717.7 & 99.1 \\
\hline BGB & $14(11)$ & 0.318 & 0.194 & 191.9 & 93.2 \\
\hline Cover & $14(10)$ & -0.141 & 0.028 & 191.4 & 93.2 \\
\hline Height & $22(14)$ & -0.957 & $<0.0001$ & 2913.4 & 99.3 \\
\hline Litter & $10(5)$ & -1.403 & 0.028 & 76.2 & 88.2 \\
\hline Nitrogen content & $4(3)$ & 0.116 & 0.205 & 11.6 & 74.1 \\
\hline Richness & $30(17)$ & 0.299 & 0.013 & 1187.6 & 97.6 \\
\hline \multicolumn{6}{|l|}{ Fauna properties } \\
\hline Invertebrate abundance & $37(13)$ & -0.319 & 0.204 & 436.2 & 91.7 \\
\hline - $\quad$ Benthos only & $12(6)$ & -0.166 & 0.722 & 219.3 & 95.0 \\
\hline - Detritivores only & $11(4)$ & -0.312 & 0.504 & 110.9 & 91.0 \\
\hline - $\quad$ Herbivores only & $9(3)$ & -1.429 & 0.030 & 92.7 & 91.4 \\
\hline - $\quad$ Predators only & $10(5)$ & -0.071 & 0.579 & 16.4 & 45.3 \\
\hline Invertebrate richness & $16(9)$ & -0.153 & $<0.0001$ & 71.2 & 78.9 \\
\hline Vertebrate abundance & $13(8)$ & 0.334 & 0.209 & 210.9 & 94.3 \\
\hline $\begin{array}{l}\text { Goose } \\
\text { abundance }\end{array}$ & $8(4)$ & 0.388 & 0.339 & 108.9 & 93.6 \\
\hline
\end{tabular}


Table A3. Results of all regression analyses. $\mathrm{n}(\mathrm{N})=$ number of entries (number of studies); $\mathrm{df}, \mathrm{F}$ and $P$ show results of ANOVA; FDR- $P=$ False Discovery Rate-adjusted $P$ value; Marg $R^{2}=$ proportion of variance explained by fixed moderator; Cond $R^{2}=$ proportion of variance explained by fixed moderator and random terms. $P$ values $<0.05$ are highlighted in bold. For continuous moderators, the model intercept (b), estimate $(\mathrm{m})$ and standard error (SE) are given. Moderators ('Mod'): stocking density ('LSU'; livestock units per hectare), duration of grazing at site ('D'; years), grazer identity ('G'; artificial, cow, sheep, mixed, other), location of study ('C'; America, Europe); dominant vegetation type tested for European studies ('V'; forbs, graminoids). Functional group ('FG') was also tested for invertebrate abundance (benthic invertebrate, herbivore, predator, detritivore) and vertebrate abundance (goose, wader).

\begin{tabular}{|c|c|c|c|c|c|c|c|c|c|c|}
\hline $\begin{array}{l}\text { Ecosystem } \\
\text { Property }\end{array}$ & Mod & $n(N)$ & df & $F$ & $P$ & FDR-P & $\begin{array}{l}\text { Marg } \\
\mathbf{R}^{2}\end{array}$ & $\begin{array}{l}\text { Cond } \\
R^{2}\end{array}$ & b & $m$ (SE) \\
\hline Bulk & LSU & $18(10)$ & $1,7.0$ & 0.00 & 0.957 & 0.957 & 0.00 & 0.98 & 0.42 & $-0.00(0.09)$ \\
\hline \multirow[t]{3}{*}{ density* } & $\mathrm{D}$ & 20(13) & 1,10 & 0.18 & 0.677 & 0.957 & 0.02 & 0.98 & 0.29 & $0.01(0.01)$ \\
\hline & G & $20(13)$ & $3,6.4$ & 2.31 & 0.170 & 0.680 & 0.40 & 0.98 & & \\
\hline & $C$ & $21(13)$ & $1,7.6$ & 0.06 & 0.820 & 0.957 & 0.00 & 0.98 & & \\
\hline \multirow[t]{4}{*}{ Soil carbon* } & LSU & 19(11) & $1,15.1$ & 0.36 & 0.555 & 0.618 & 0.01 & 0.76 & -0.07 & $0.08(0.14)$ \\
\hline & $\mathrm{D}$ & $23(14)$ & $1,8.9$ & 0.27 & 0.618 & 0.618 & 0.02 & 0.73 & 0.08 & $-0.00(0.00)$ \\
\hline & G & $24(14)$ & $2,4.9$ & 1.31 & 0.349 & 0.618 & 0.20 & 0.73 & & \\
\hline & C & $27(16)$ & $1,14.8$ & 9.06 & 0.009 & 0.036 & 0.33 & 0.87 & & \\
\hline \multirow[t]{2}{*}{ Redox* } & $\mathrm{D}$ & $12(6)$ & $1,3.7$ & 0.02 & 0.898 & 0.898 & 0.00 & 0.90 & -0.45 & $-0.00(0.02)$ \\
\hline & G & $10(5)$ & $2,4.9$ & 3.81 & 0.100 & 0.200 & 0.54 & 0.86 & & \\
\hline \multirow[t]{4}{*}{ Salinity* } & LSU & $14(7)$ & $1,11.0$ & 5.84 & 0.034 & 0.136 & 0.33 & 0.32 & 0.78 & $-0.63(0.26)$ \\
\hline & $\mathrm{D}$ & $16(8)$ & $1,7.1$ & 0.04 & 0.846 & 0.846 & 0.00 & 0.35 & 0.21 & $-0.00(0.01)$ \\
\hline & G & $15(8)$ & $1,7.5$ & 0.15 & 0.713 & 0.846 & 0.01 & 0.37 & & \\
\hline & C & $17(9)$ & $1,7.1$ & 4.61 & 0.068 & 0.136 & 0.25 & 0.33 & & \\
\hline \multirow[t]{5}{*}{ AGB } & LSU & $18(10)$ & $1,15.4$ & 7.76 & 0.014 & 0.070 & 0.32 & 0.81 & -0.43 & $-0.70(0.25)$ \\
\hline & $\mathrm{D}$ & 29(15) & $1,17.2$ & 1.56 & 0.229 & 0.393 & 0.06 & 0.19 & -0.86 & $-0.02(0.01)$ \\
\hline & G & $32(17)$ & $4,16.7$ & 0.79 & 0.550 & 0.550 & 0.12 & 0.31 & & \\
\hline & C & $35(19)$ & $1,21.8$ & 0.56 & 0.462 & 0.550 & 0.02 & 0.18 & & \\
\hline & V & $14(7)$ & $1,12.0$ & 1.56 & 0.236 & 0.393 & 0.11 & 0.11 & & \\
\hline \multirow[t]{3}{*}{ BGB* } & LSU & $10(7)$ & $1,2.0$ & 10.14 & 0.085 & 0.128 & 0.01 & 0.99 & 0.65 & $-0.11(0.03)$ \\
\hline & $\mathrm{D}$ & $11(8)$ & $1,6.0$ & 2.24 & 0.186 & 0.186 & 0.27 & 0.99 & 0.14 & $0.02(0.02)$ \\
\hline & G & $14(9)$ & $2,5.9$ & 6.25 & 0.035 & 0.105 & 0.59 & 0.95 & & \\
\hline \multirow{5}{*}{$\begin{array}{l}\text { Vegetation } \\
\text { cover }\end{array}$} & LSU & $14(8)$ & $1,11.0$ & 3.31 & 0.096 & 0.240 & 0.18 & 0.79 & 0.00 & $-0.17(0.10)$ \\
\hline & $\mathrm{D}$ & $14(8)$ & $1,3.6$ & 2.57 & 0.193 & 0.241 & 0.21 & 0.98 & 0.08 & $-0.02(0.01)$ \\
\hline & G & $23(15)$ & $2,7.8$ & 2.45 & 0.149 & 0.241 & 0.48 & 0.97 & & \\
\hline & C & $22(14)$ & $1,11.5$ & 0.09 & 0.771 & 0.771 & 0.01 & 0.97 & & \\
\hline & V & $10(7)$ & $1,3.3$ & 9.87 & 0.045 & 0.225 & 0.21 & 0.95 & & \\
\hline \multirow{4}{*}{$\begin{array}{l}\text { Canopy } \\
\text { height* }\end{array}$} & LSU & $32(16)$ & $1,22.4$ & 12.91 & 0.002 & 0.008 & 0.28 & 0.50 & -0.14 & $-1.00(0.28)$ \\
\hline & $\mathrm{D}$ & $24(12)$ & $1,6.6$ & 6.28 & 0.043 & 0.086 & 0.22 & 0.32 & -0.43 & $-0.03(0.01)$ \\
\hline & G & $37(18)$ & $2,9.3$ & 0.41 & 0.674 & 0.679 & 0.04 & 0.52 & & \\
\hline & V & $19(10)$ & $1,7.7$ & 0.19 & 0.679 & 0.679 & 0.03 & 0.88 & & \\
\hline \multirow{4}{*}{$\begin{array}{l}\text { Litter } \\
\text { biomass* }\end{array}$} & LSU & $12(7)$ & $1,8.9$ & 0.19 & 0.677 & 0.677 & 0.01 & 0.57 & -1.33 & $-0.32(0.73)$ \\
\hline & $\mathrm{D}$ & $16(8)$ & $1,6.6$ & 5.73 & 0.051 & 0.204 & 0.37 & 0.66 & -0.52 & $-0.05(0.02)$ \\
\hline & G & $14(7)$ & $2,2.4$ & 1.08 & 0.463 & 0.617 & 0.25 & 0.72 & & \\
\hline & C & $17(9)$ & $1,6.3$ & 1.84 & 0.222 & 0.444 & 0.18 & 0.69 & & \\
\hline \multirow{2}{*}{$\begin{array}{l}\text { Nitrogen } \\
\text { content* }\end{array}$} & D & $11(6)$ & $1,9.0$ & 0.63 & 0.446 & 0.892 & 0.06 & 0.06 & 0.12 & $-0.00(0.00)$ \\
\hline & G & $11(6)$ & $2,2.3$ & 0.01 & 0.994 & 0.994 & 0.00 & 0.66 & & \\
\hline \multirow{5}{*}{$\begin{array}{l}\text { Vegetation } \\
\text { richness }\end{array}$} & LSU & $29(16)$ & $1,25.3$ & 0.91 & 0.350 & 0.485 & 0.02 & 0.59 & 0.31 & $-0.15(0.16)$ \\
\hline & D & $46(20)$ & $1,12.5$ & 1.69 & 0.217 & 0.485 & 0.05 & 0.18 & 0.24 & $-0.00(0.00)$ \\
\hline & G & $52(24)$ & $2,15.6$ & 0.40 & 0.678 & 0.678 & 0.02 & 0.68 & & \\
\hline & C & $54(26)$ & $1,31.3$ & 0.77 & 0.388 & 0.485 & 0.02 & 0.15 & & \\
\hline & $\mathrm{V}$ & $23(14)$ & $1,21.0$ & 5.05 & 0.036 & 0.180 & 0.19 & 0.19 & & \\
\hline
\end{tabular}


Appendix A

\begin{tabular}{|c|c|c|c|c|c|c|c|c|c|c|}
\hline \multirow{5}{*}{$\begin{array}{l}\text { Invert } \\
\text { abundance* }\end{array}$} & LSU & 30(9) & $1,12.9$ & 0.55 & 0.472 & 0.590 & 0.01 & 0.55 & -0.50 & $-0.27(0.36)$ \\
\hline & $\mathrm{D}$ & $31(10)$ & $1,13.6$ & 4.60 & 0.051 & 0.255 & 0.15 & 0.44 & -0.13 & $-0.04(0.02)$ \\
\hline & G & 39(13) & $2,9.1$ & 2.18 & 0.169 & 0.282 & 0.13 & 0.51 & & \\
\hline & C & $42(15)$ & $1,10.3$ & 0.05 & 0.827 & 0.827 & 0.00 & 0.42 & & \\
\hline & FG & $34(12)$ & $3,21.6$ & 0.15 & 0.135 & 0.282 & 0.15 & 0.15 & & \\
\hline \multirow{5}{*}{$\begin{array}{l}\text { Invert } \\
\text { richness }\end{array}$} & LSU & $15(7)$ & $1,10.0$ & 0.29 & 0.603 & 0.890 & 0.03 & 0.03 & -0.22 & $-0.11(0.21)$ \\
\hline & $D$ & $11(5)$ & $1,9.0$ & 0.00 & 0.963 & 0.963 & 0.00 & 0.00 & -0.37 & $0.00(0.01)$ \\
\hline & G & $16(8)$ & $1,4.9$ & 0.15 & 0.712 & 0.890 & 0.01 & 0.05 & & \\
\hline & C & $19(10)$ & $1,17.0$ & 0.36 & 0.556 & 0.890 & 0.02 & 0.02 & & \\
\hline & V & $10(6)$ & $1,8.0$ & 1.31 & 0.286 & 0.890 & 0.13 & 0.13 & & \\
\hline \multirow{5}{*}{$\begin{array}{l}\text { Vertebrate } \\
\text { abundance* }\end{array}$} & LSU & $15(7)$ & $1,11.0$ & 0.35 & 0.568 & 0.568 & 0.03 & 0.03 & 0.99 & $-0.49(0.82)$ \\
\hline & $\mathrm{D}$ & $13(7)$ & $1,6.5$ & 5.79 & 0.050 & 0.250 & 0.22 & 0.70 & 0.13 & $0.04(0.01)$ \\
\hline & G & $22(10)$ & $1,3.9$ & 0.98 & 0.381 & 0.568 & 0.08 & 0.31 & & \\
\hline & $C$ & $26(12)$ & $1,3.7$ & 0.59 & 0.488 & 0.568 & 0.03 & 0.27 & & \\
\hline & FG & $18(7)$ & $1,4.3$ & 0.58 & 0.486 & 0.568 & 0.05 & 0.28 & & \\
\hline
\end{tabular}

* Some moderators not tested due to lack of data 


\section{Figure A1.}

Forest plots and funnel plots for all ecosystem properties. In funnel plots, larger, more precise studies (i.e. with a small standard error) appear towards the top of the graph. Where there is pronounced asymmetry, e.g. all small studies report strongly negative effects, this indicates publication bias, perhaps because smaller studies without statistically significant effects were not published. In these analyses, there were indications that the smaller studies (those with a larger standard error) had a bias towards reporting negative results for plant cover, plant richness and redox.

a) Aboveground biomass

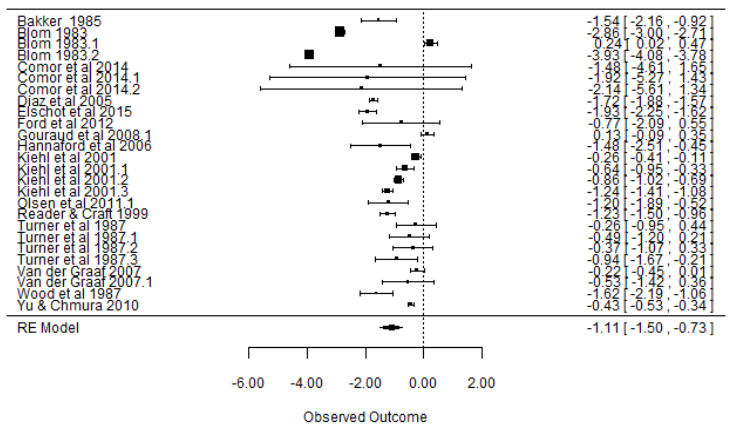

b) Belowground biomass

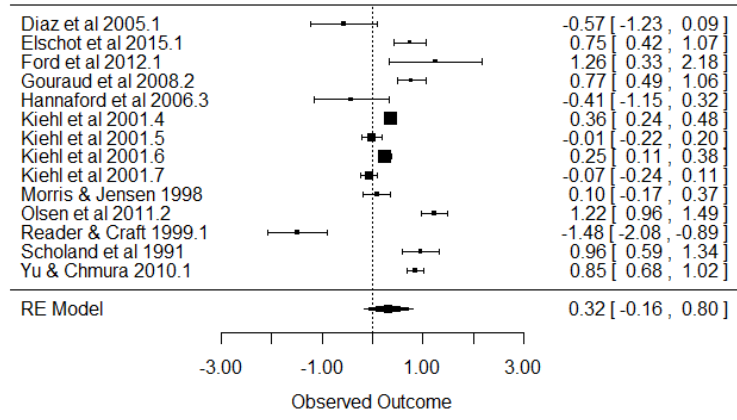

c) Plant cover
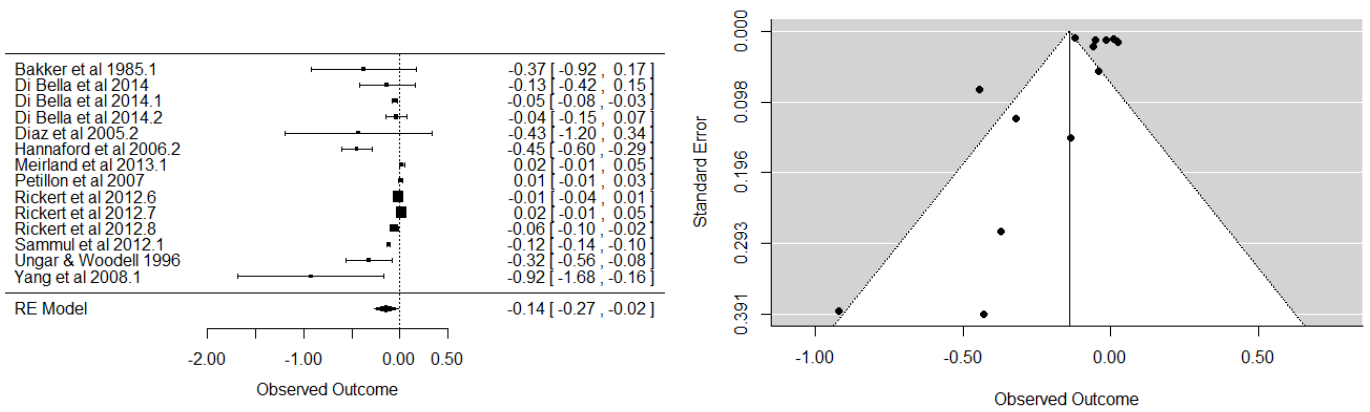
d) Canopy height

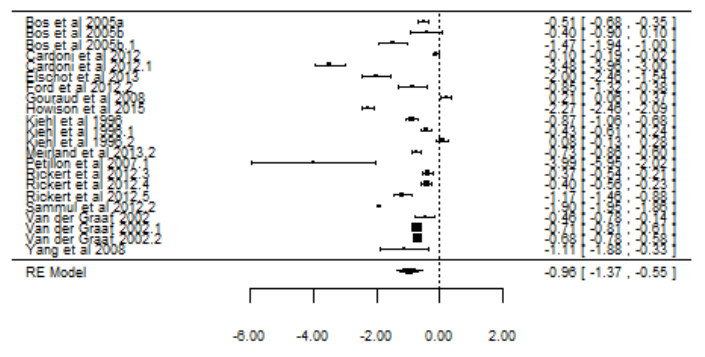

Observed Outcome

e) Litter
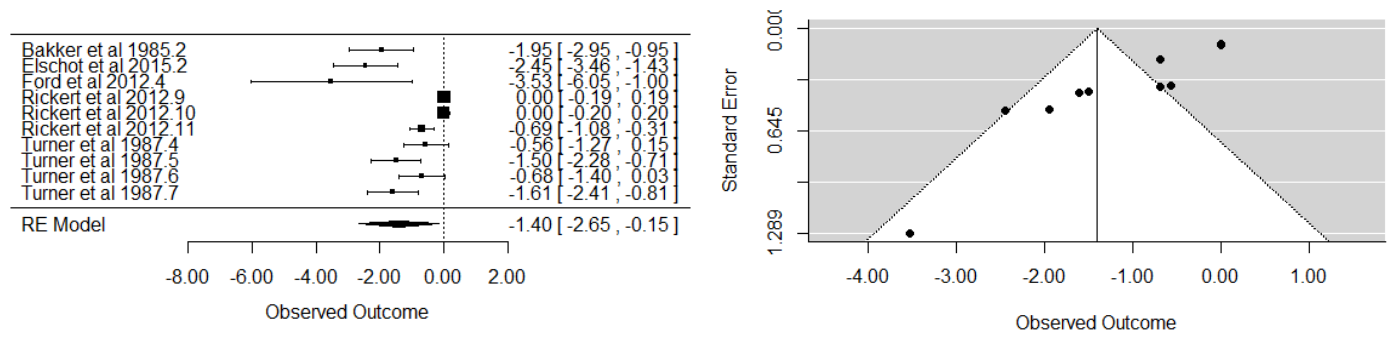

\section{f) Nitrogen content}
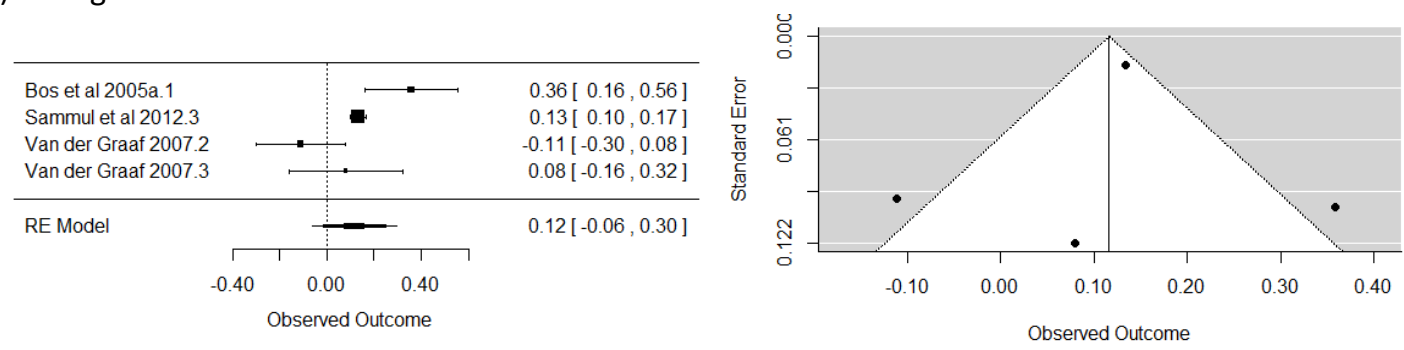

g) Plant richness
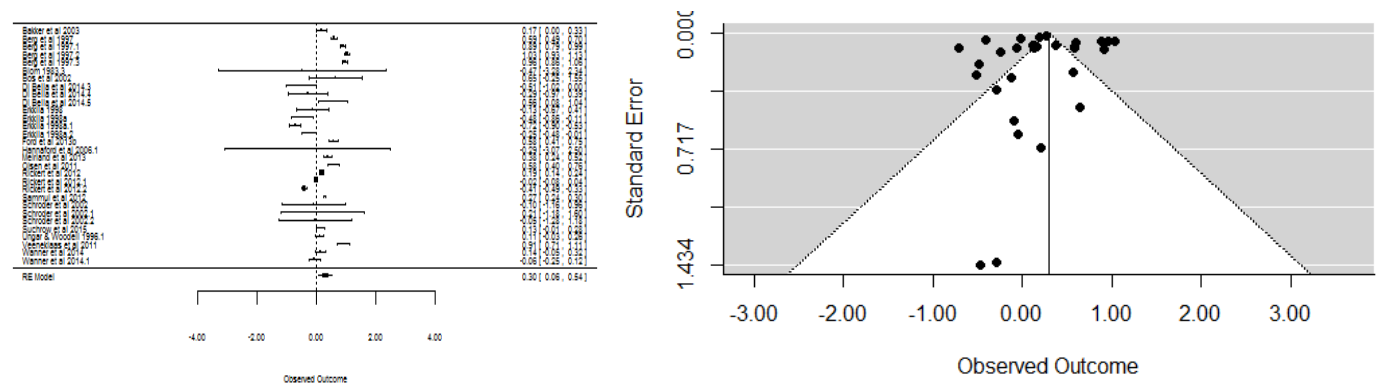

h) Accretion
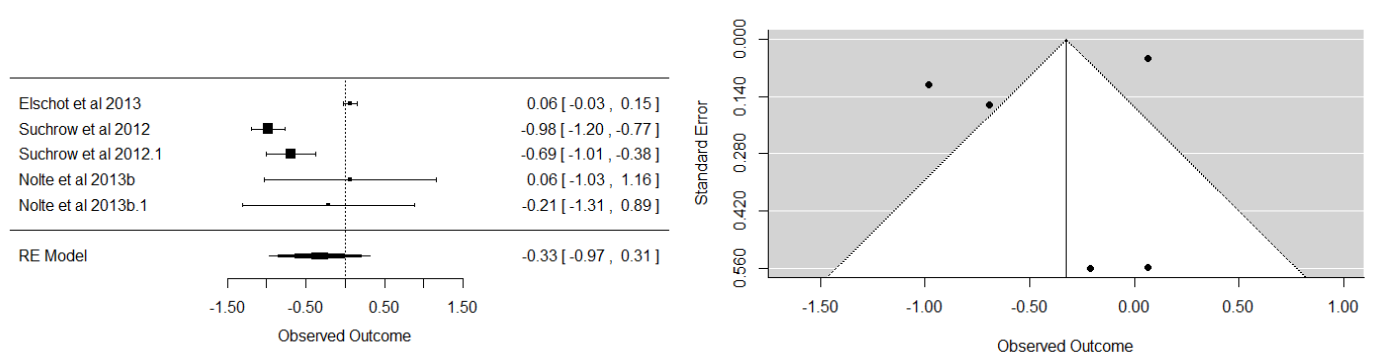
i) Bulk density
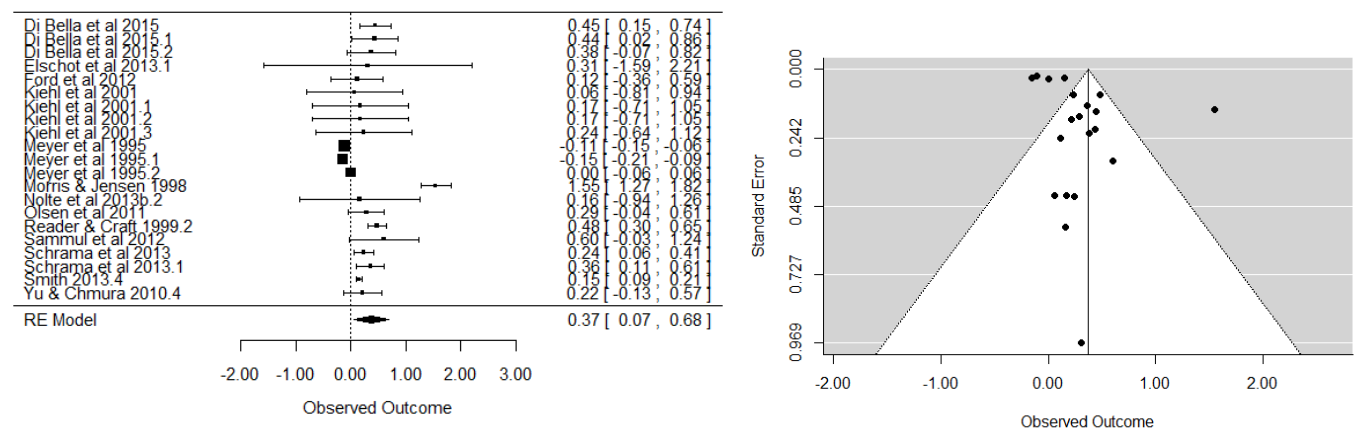

j) Soil carbon
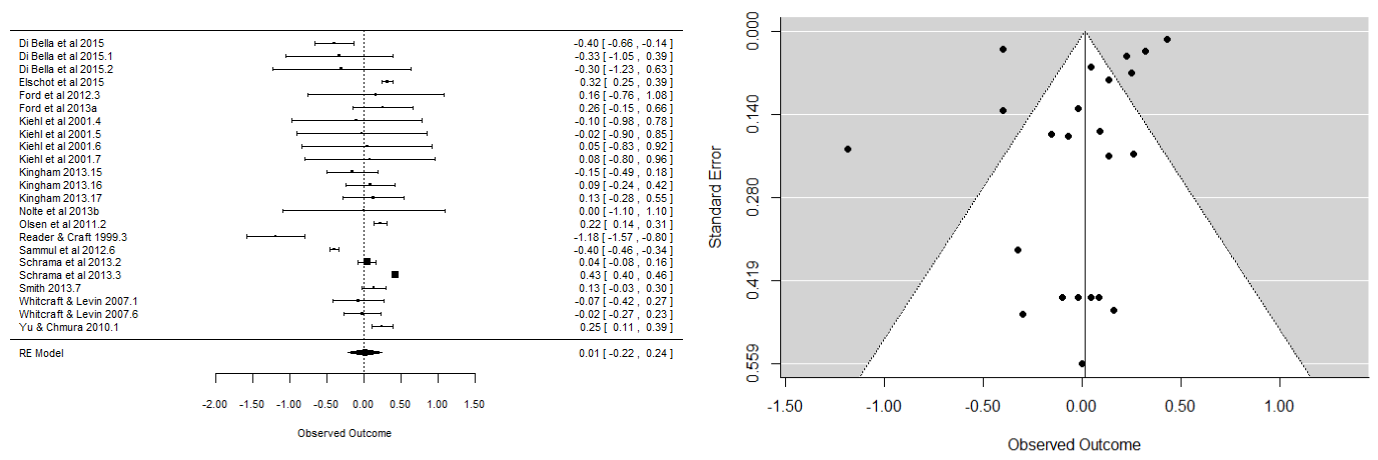

k) $\mathrm{pH}$
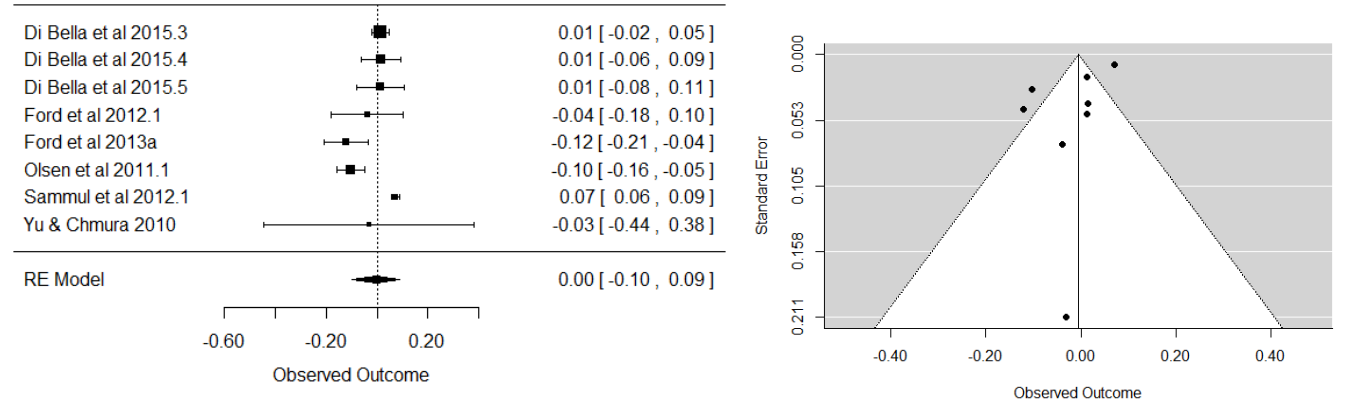

I) Redox
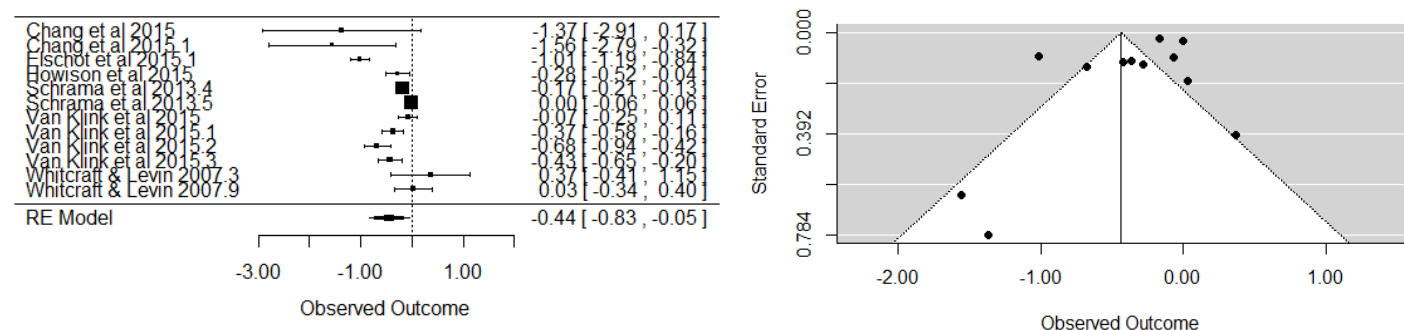
m) Salinity
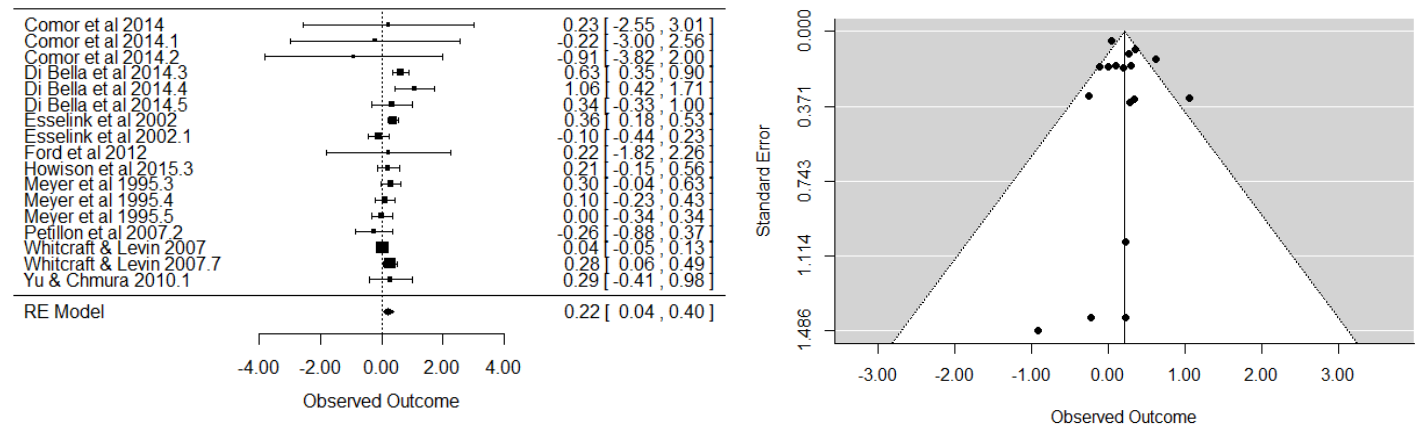

n) Soil temperature
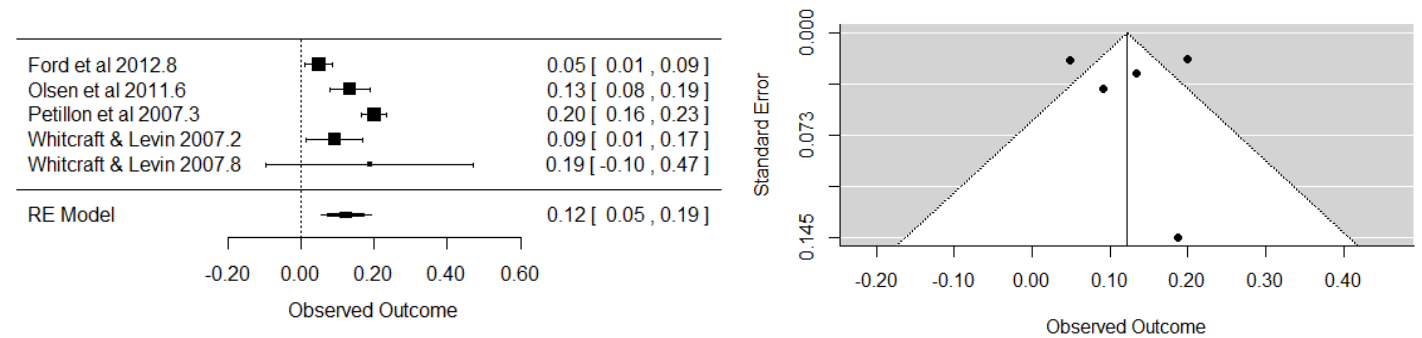

o) Invertebrate abundance
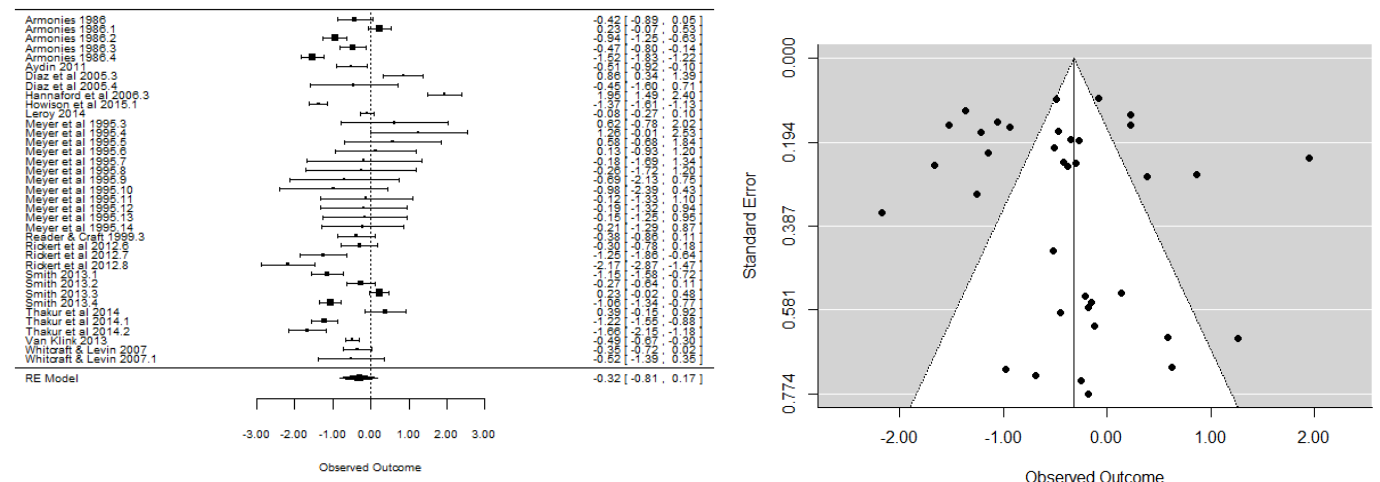

p) Invertebrate richness
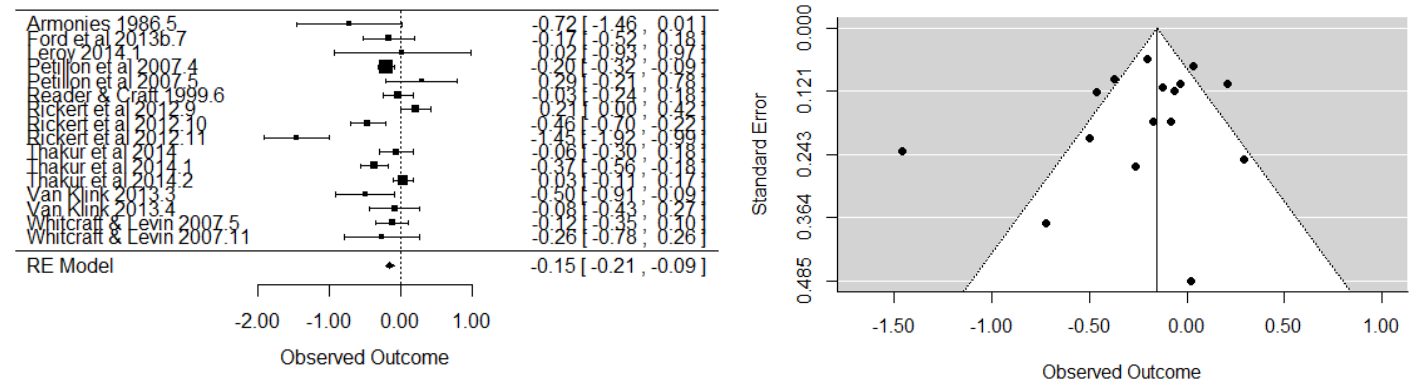

q) Benthos abundance

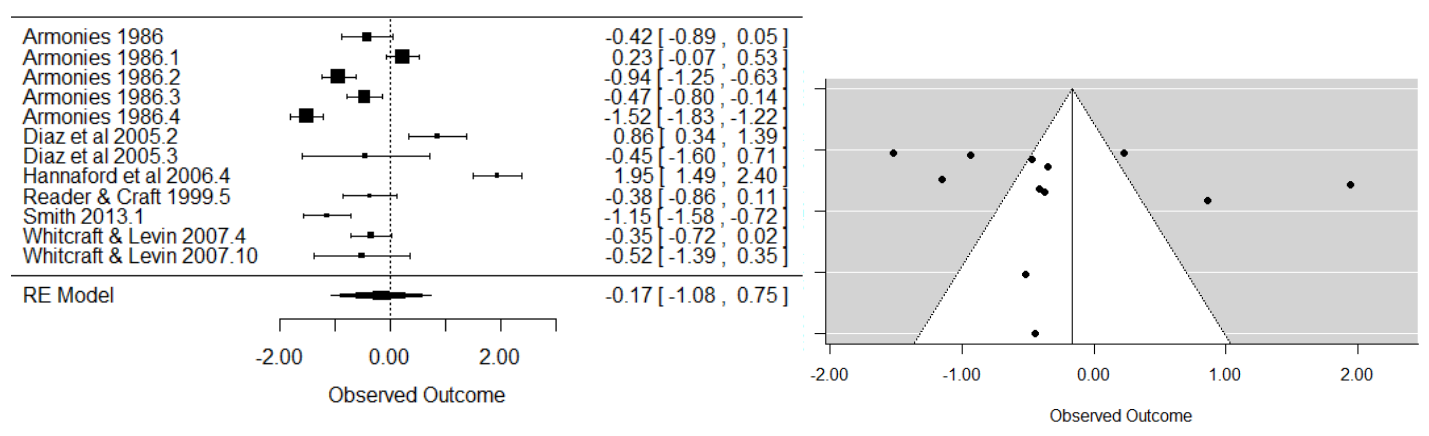


r) Predator abundance

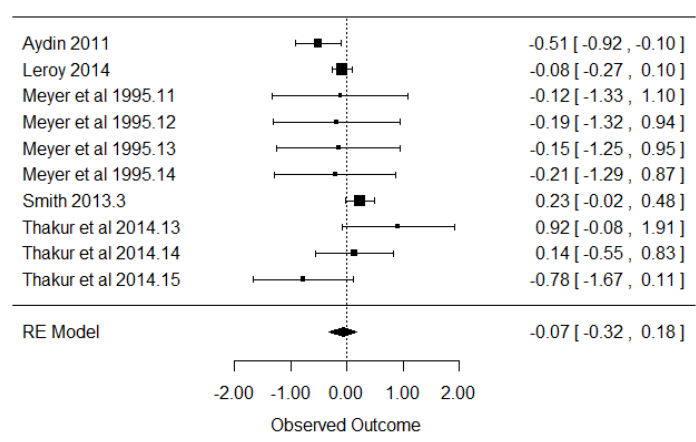

s) Detritivore abundance

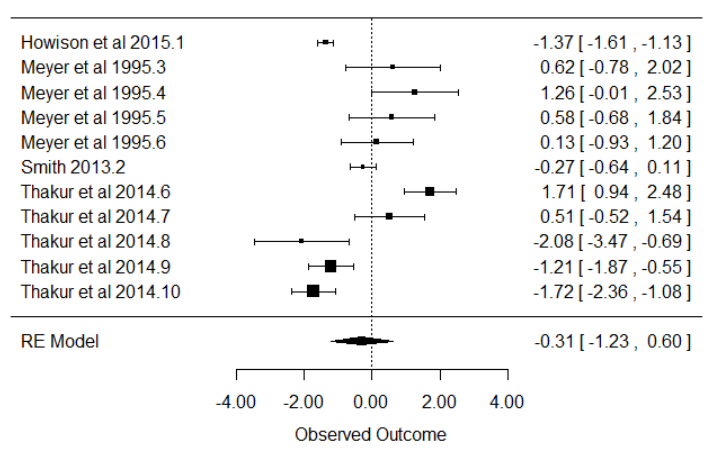

t) Herbivore abundance

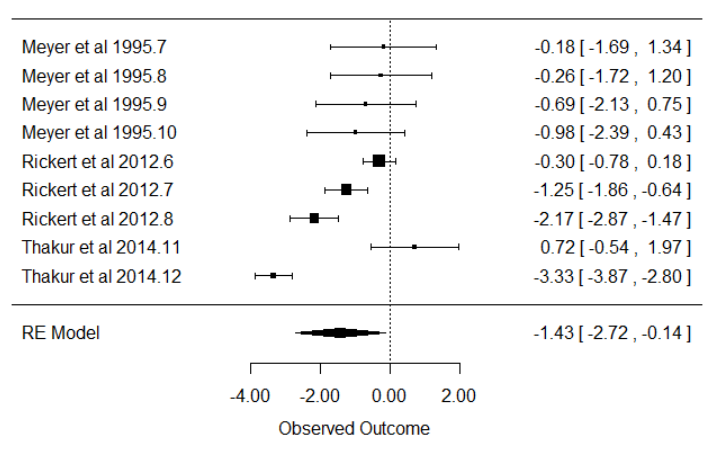

u) Vertebrate abundance

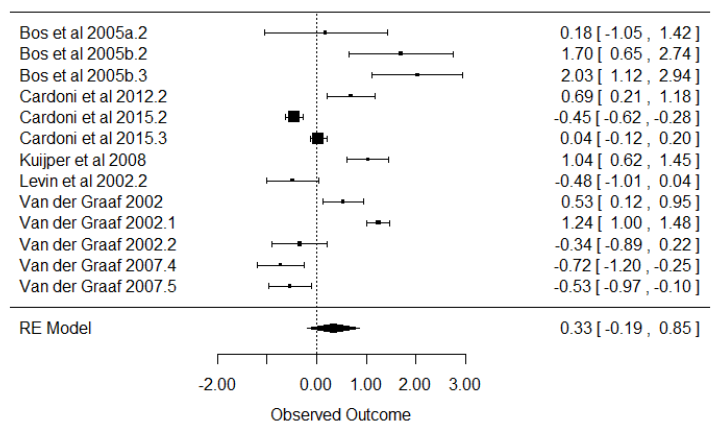

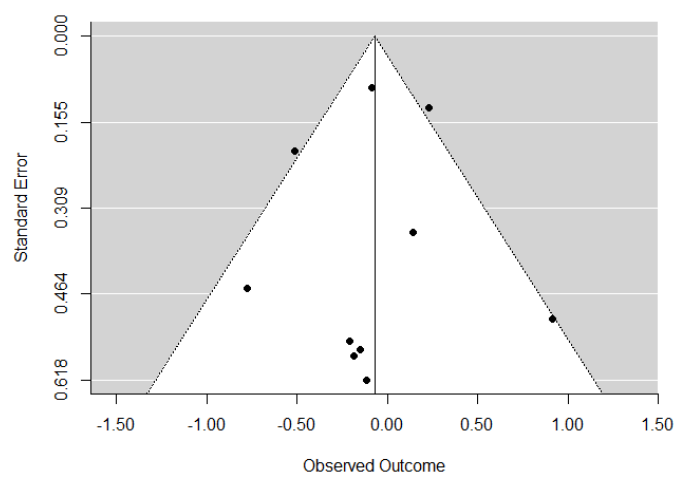
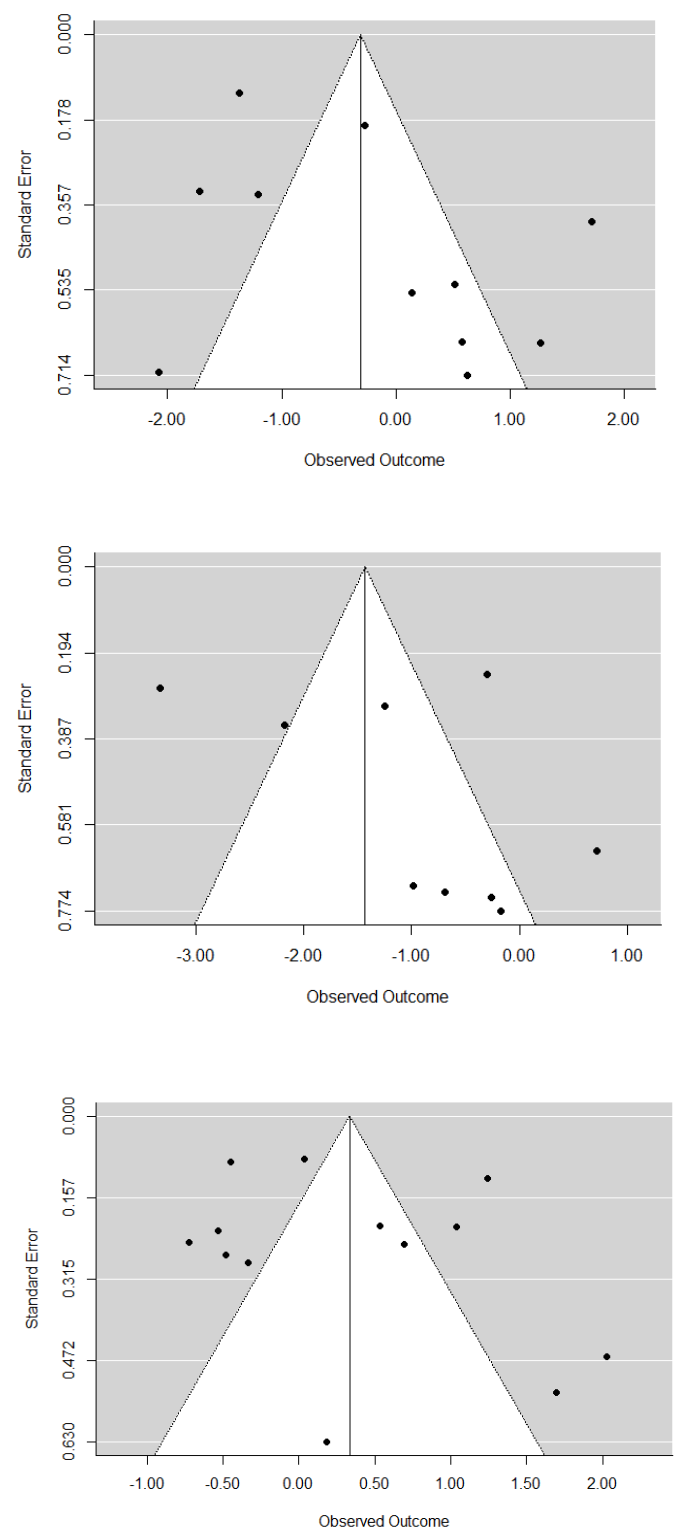

Fig. A1. Forest plots (left) and funnel plots (right) for each Ecosystem Property (a - u, indicated above graph). 
Figure A2.

Results of coded meta-analysis.

a)

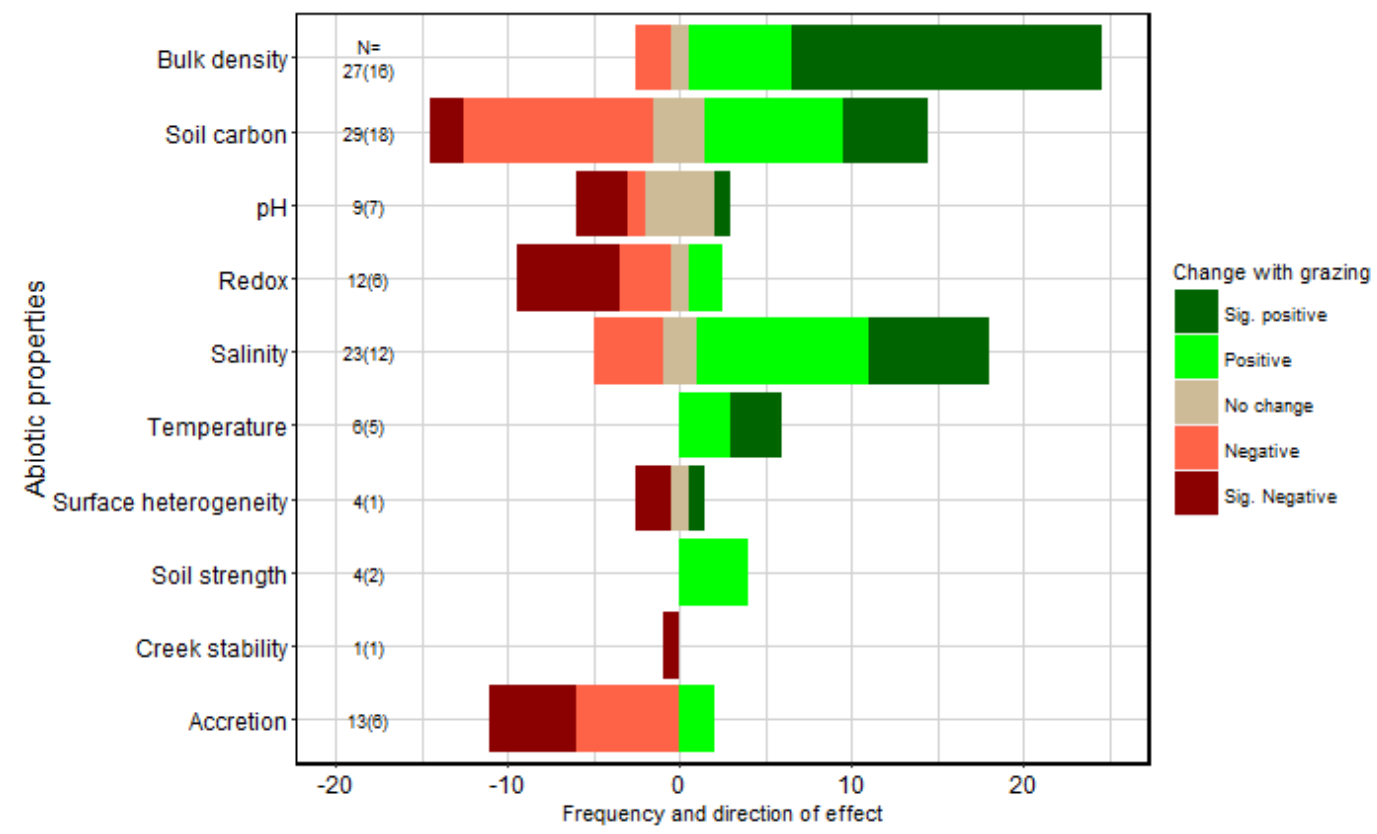

b)

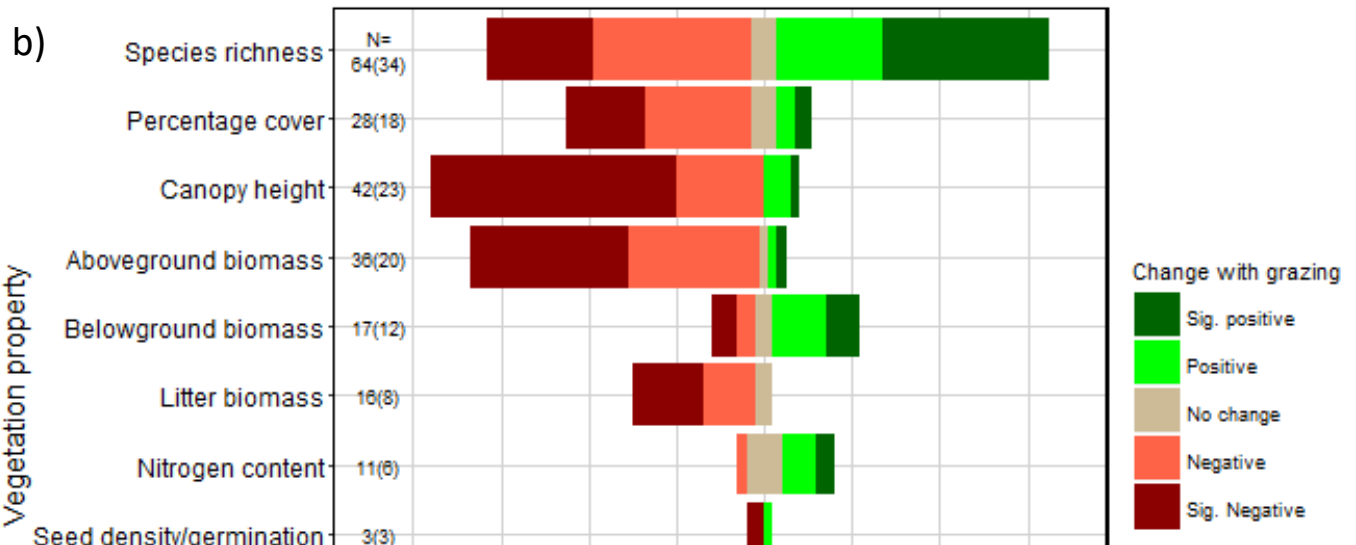
\begin{tabular}{|l|l|l|l|l|l|l|l|} 
Seed density/germination & $3(3)$ & & & & Sig. Negative \\
\hline
\end{tabular}

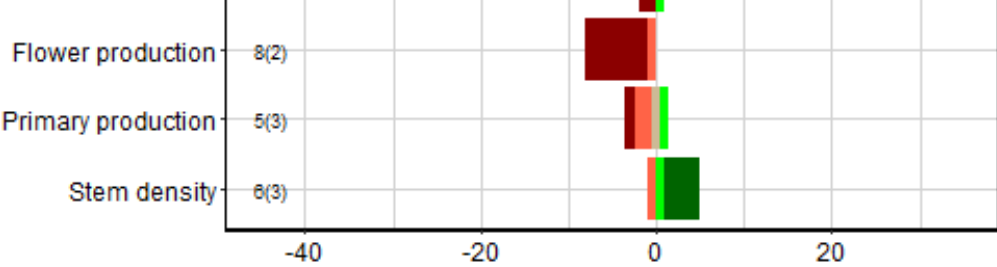

Frequency and direction of effect

c)

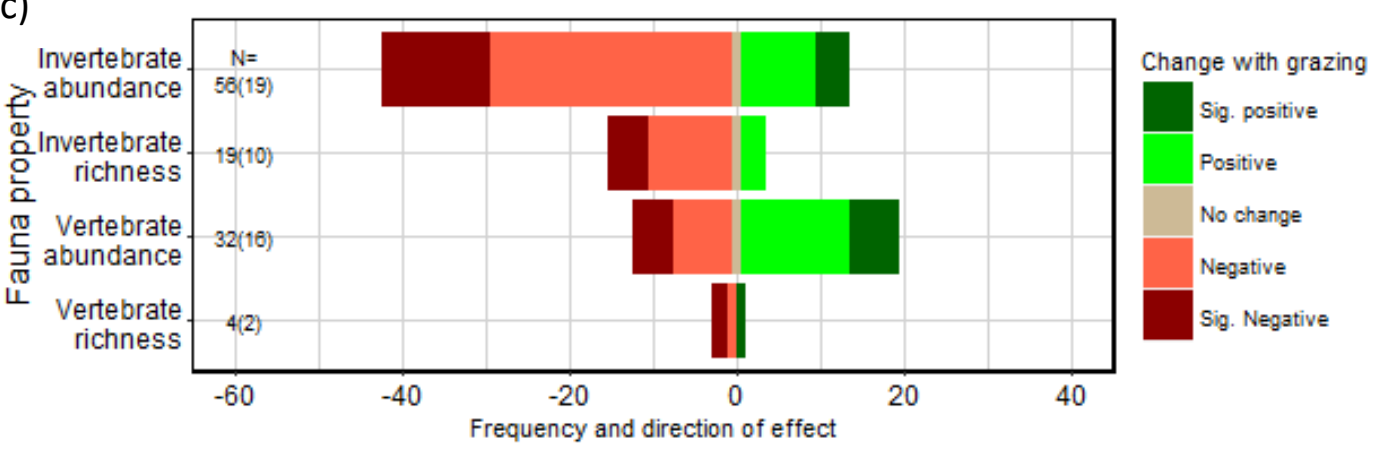



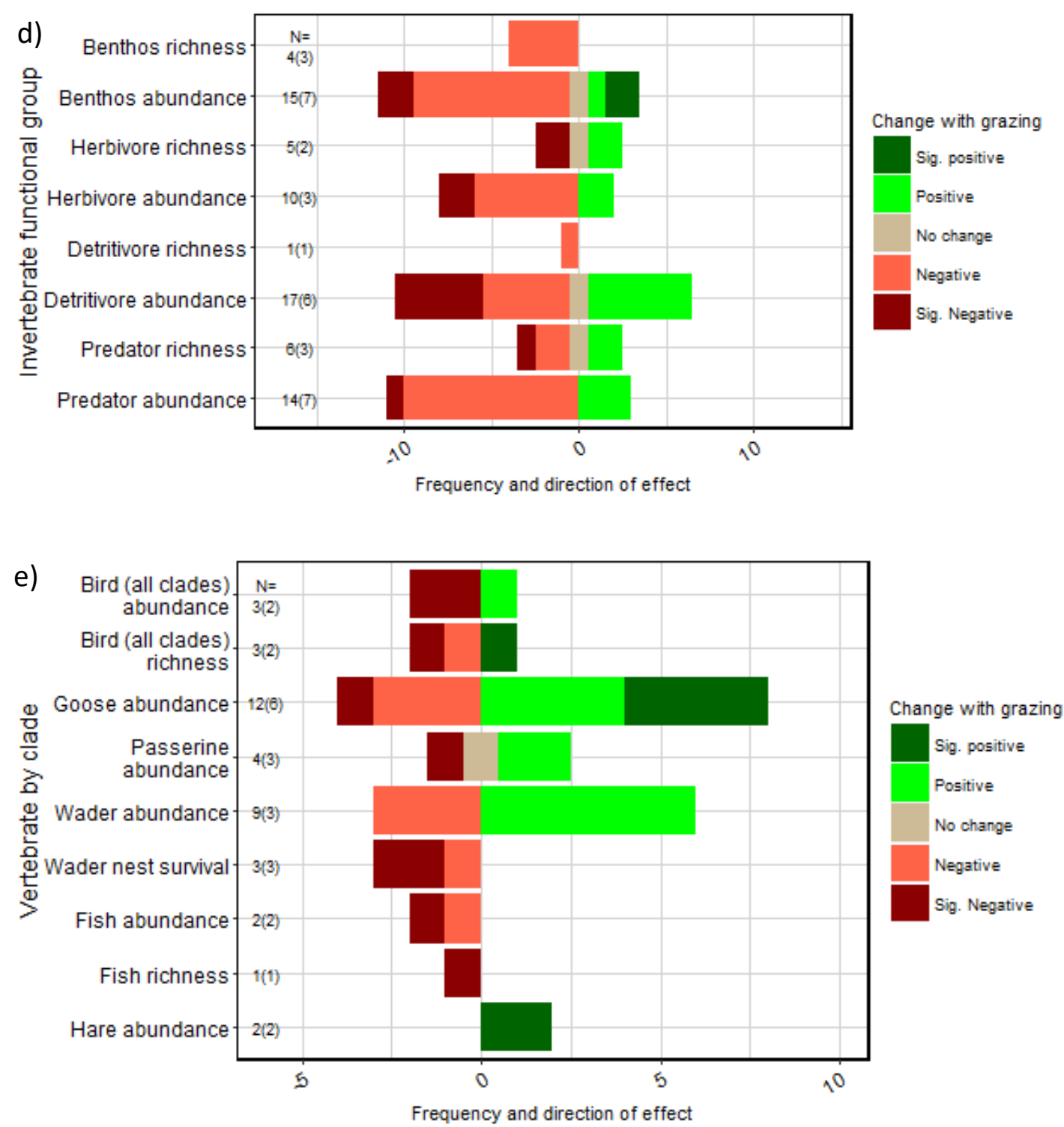

Fig. A2. Count of entries reporting response of ecosystem properties to grazing for a) soil properties, b) vegetation properties, c) fauna properties, d) invertebrates by functional group and e) vertebrates by clade. Changes classified as significantly (sig.) positive, positive, no change, negative or sig. negative. 
Figure A3.

Ecosystem Properties for which the grazing effect is moderated by grazing management (stocking density, duration, identity of grazer), identified using linear regression analysis (moderators significant at $P<0.05$ ).

a)

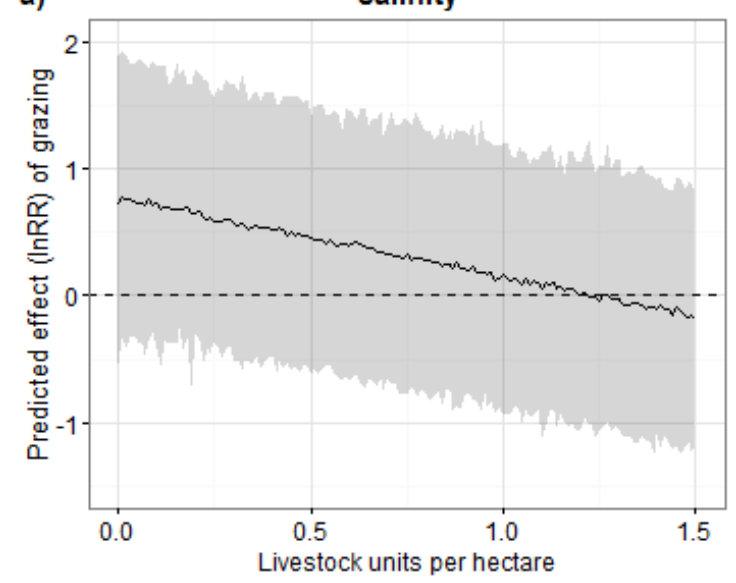

c)

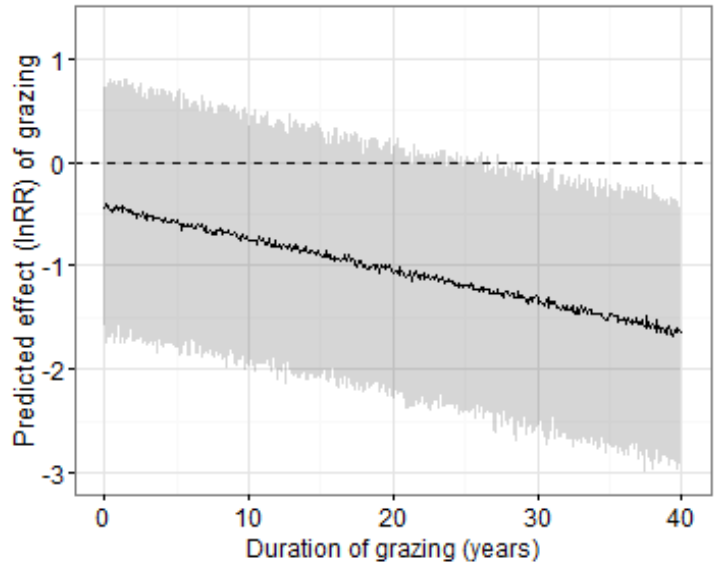

e) b)
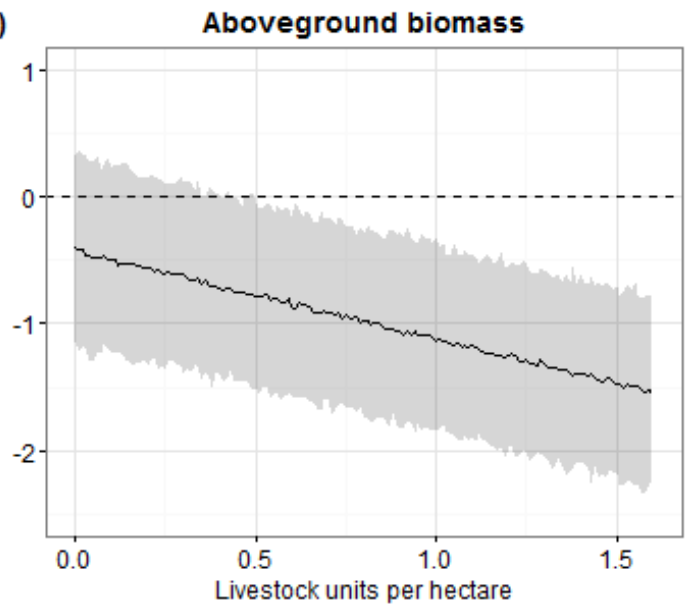

d)

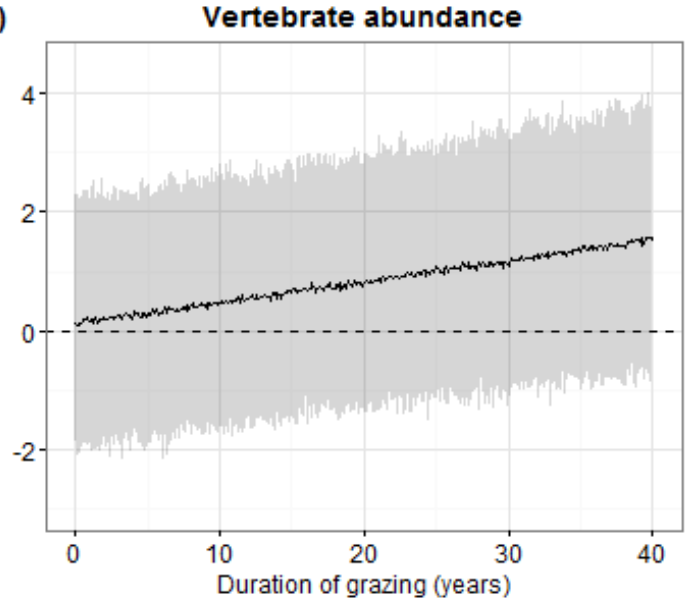

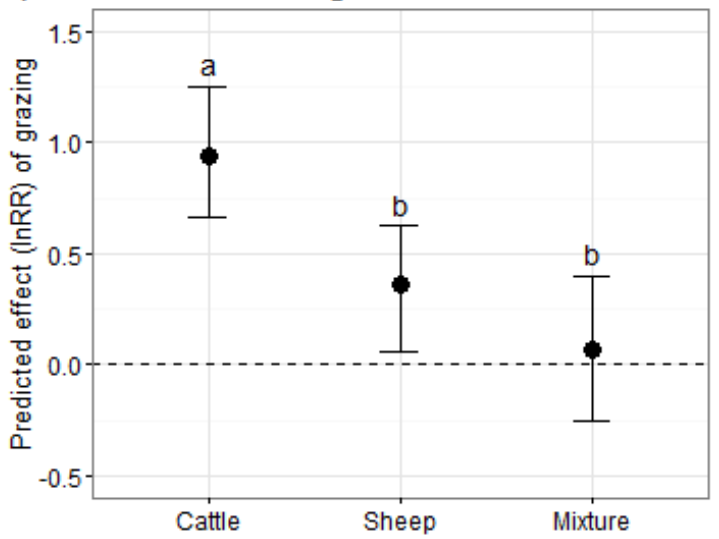

Fig. A3. Predicted moderating effects of stocking density (livestock units per hectare) on a) salinity and b) aboveground biomass; duration of grazing on c) canopy height and d) vertebrate abundance; and e) grazer identity on belowground biomass, with $95 \%$ Prediction Intervals. 


\section{Figure A4}

Ecosystem Properties for which the grazing effect is moderated by dominant vegetation type (analysed for European studies only), identified using linear regression analysis (moderators significant at $P<0.05$ ).
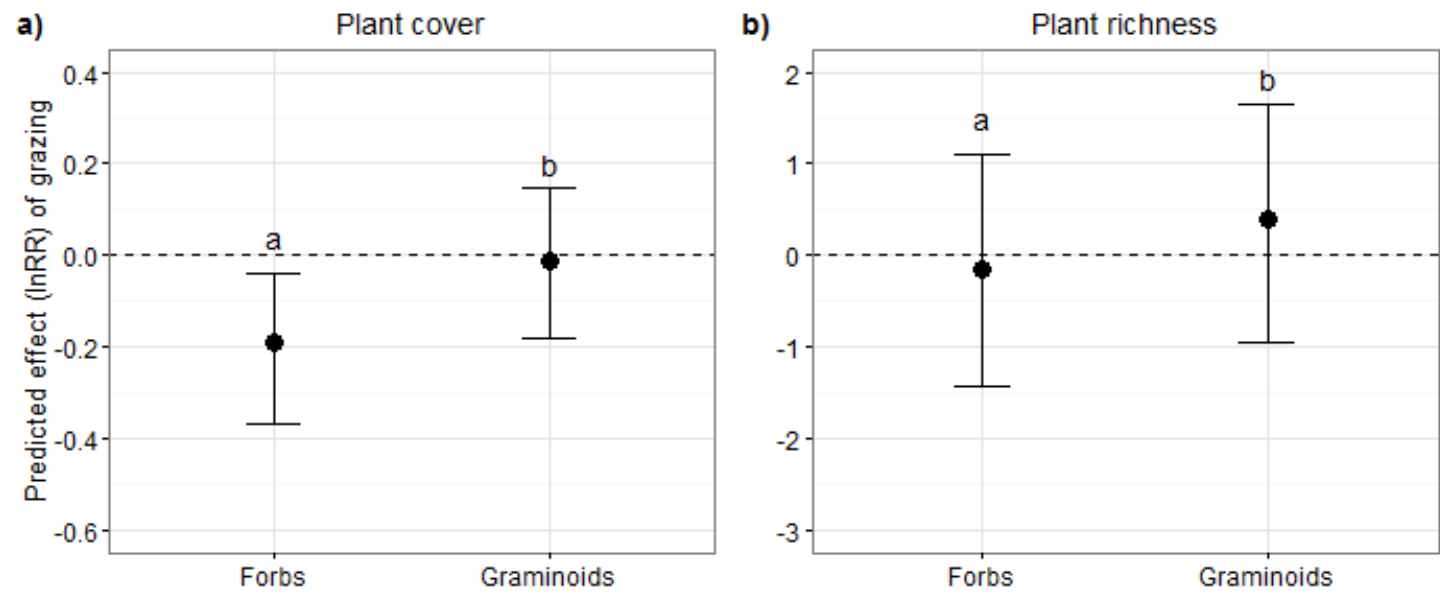

Fig. A4. Predicted moderating effects of grazing on a) percentage cover, and b) plant richness in different vegetation types in European salt marshes, with 95\% Prediction Intervals. 


\section{Appendix B: Supporting information for Chapter 3}

\section{Contents}

Table B1. Visual indicators used to allocate qualitative grazing levels

Table B2. Effect of grazing on ecosystem properties in observational survey

Table B3. Effect of grazing intensity on ecosystem properties in observational survey

Table B4. Baseline comparisons, control vs experimental plots

Table B5. Baseline comparisons, control vs reference points

Table B6. Effect of grazing on ecosystem properties after 18 months

Table B7. Effect of grazing on ecosystem properties at each time point

Table B8. Effect of grazing on recovery from damage

Figure B1. Soil texture classes triangle

Figure B2. Raw species data for observational survey marshes 
Table B1. Visual indicators used to allocate qualitative grazing levels. Each descriptor is not absolute, but was used in combination and alongside information from site managers to allocate the best representative grazing level to each marsh.

\begin{tabular}{|c|c|c|c|c|}
\hline Indicator & 0: Ungrazed & 1: Lightly grazed & 2: Moderately grazed & 3: Heavily grazed \\
\hline Animal presence & None & $\begin{array}{l}\text { Area accessible to animals } \\
\text { but no more than a few } \\
\text { animals present }\end{array}$ & $\begin{array}{l}\text { Small band (e.g. 5-10) } \\
\text { present }\end{array}$ & $\begin{array}{l}\text { Many animals (>10 } \\
\text { horses) present }\end{array}$ \\
\hline Droppings & None & Infrequently seen & Commonly seen & Abundant across area \\
\hline $\begin{array}{l}\text { Damage to } \\
\text { ground }\end{array}$ & None & $\begin{array}{l}\text { Hoofprints rarely seen. } \\
\text { Very little bare ground }\end{array}$ & $\begin{array}{l}\text { Hoofprints/poaching } \\
\text { commonly seen. Some } \\
\text { bare ground }\end{array}$ & $\begin{array}{l}\text { Ground is badly } \\
\text { poached. Areas of bare } \\
\text { ground common }\end{array}$ \\
\hline $\begin{array}{l}\text { Sward height and } \\
\text { damage to } \\
\text { vegetation }\end{array}$ & $\begin{array}{l}\text { Sward is long with } \\
\text { complex structure, } \\
\text { no visible cropping }\end{array}$ & $\begin{array}{l}\text { Majority of sward is long } \\
\text { with complex structure, } \\
\text { little visible cropping (i.e. } \\
\text { few blunt ends to grass) }\end{array}$ & $\begin{array}{l}\text { Sward is moderate } \\
\text { length, or a matrix of } \\
\text { short and longer areas, } \\
\text { with some visibly } \\
\text { cropped areas }\end{array}$ & $\begin{array}{l}\text { Majority of vegetation } \\
\text { cropped to a short, } \\
\text { uniform sward }\end{array}$ \\
\hline
\end{tabular}


Table B2. Effect of grazing on ecosystem properties in observational survey. Results of (generalised) linear mixed models to test the effect grazing on multiple ecosystem properties. Coefficients (coef) and standard errors (SE) are given both for the original modelled scale and for models using standardised Z-transformed data (Z-coef). Coefficients for 'Grazed' and 'Season' show the effect of grazing relative to ungrazed, and spring relative to fall. All models were fitted with a random effect of marsh nested in site. Marginal (Marg) $R^{2}$ values relate to fixed effects only, Conditional (Cond) $R^{2}$ values relate to fixed + random effects.

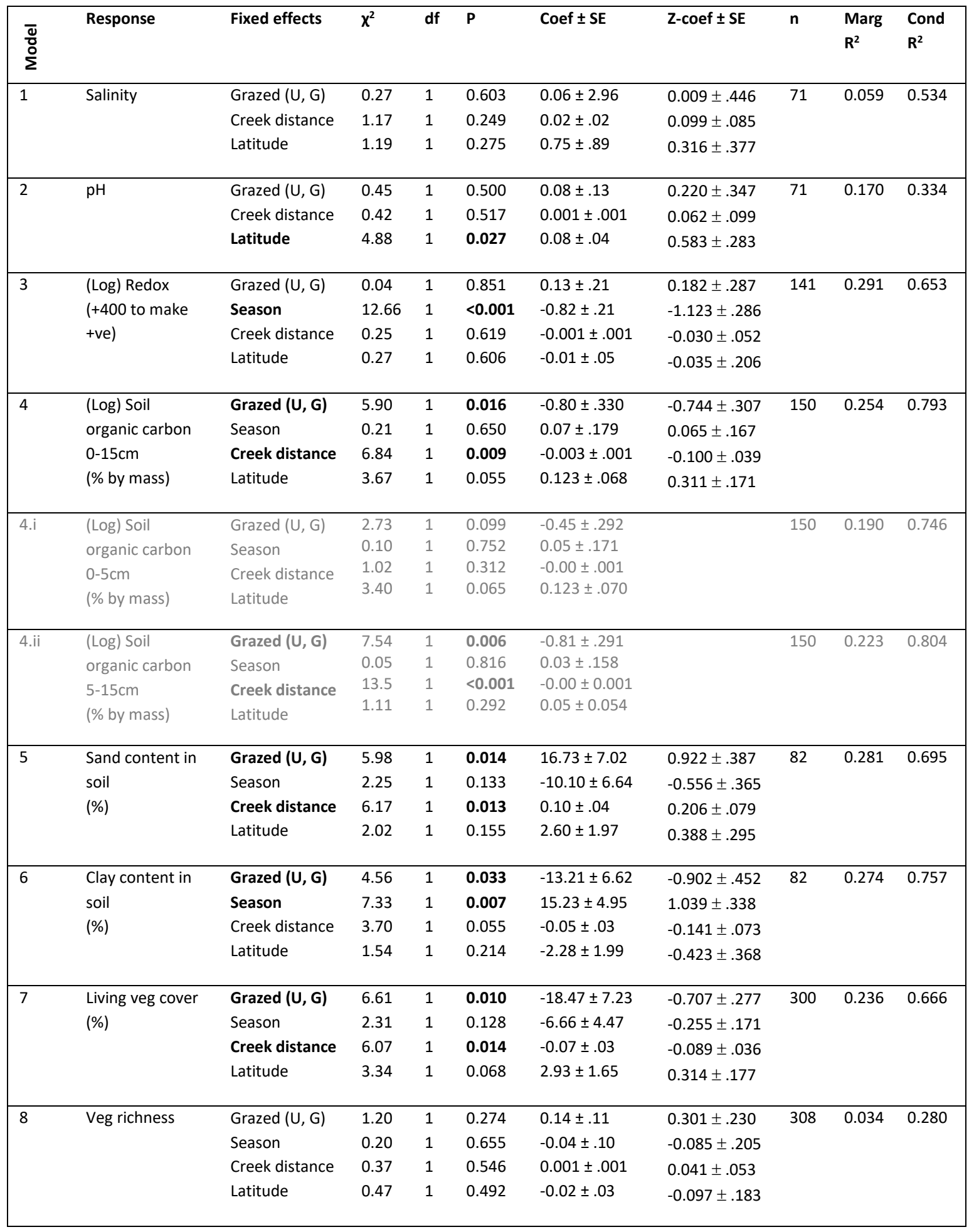


Table B2 cont.

\begin{tabular}{|c|c|c|c|c|c|c|c|c|c|c|}
\hline $\begin{array}{l}\overline{0} \\
\text { ¿ } \\
\Sigma\end{array}$ & Response & Fixed effects & $x^{2}$ & df & $\mathbf{P}$ & Coef \pm SE & Z-coef $\pm S E$ & $n$ & $\begin{array}{l}\text { Marg } \\
\mathbf{R}^{2}\end{array}$ & $\begin{array}{l}\text { Cond } \\
R^{2}\end{array}$ \\
\hline$a$ & $\begin{array}{l}\text { Spartina cover } \\
\text { (Binomial } \\
\text { GLMM: } \\
1=100 \% \\
\text { Spartina, } \\
0=<100 \% \\
\text { Spartina) }\end{array}$ & $\begin{array}{l}\text { Grazed }(U, G) \\
\text { Season } \\
\text { Creek distance } \\
\text { Latitude }\end{array}$ & $\begin{array}{l}1.48 \\
0.11 \\
1.43 \\
0.35\end{array}$ & $\begin{array}{l}1 \\
1 \\
1 \\
1\end{array}$ & $\begin{array}{l}0.224 \\
0.736 \\
0.232 \\
0.556\end{array}$ & $\begin{array}{l}-0.84 \pm .65 \\
0.22 \pm .62 \\
-0.006 \pm .005 \\
0.11 \pm .19\end{array}$ & & 306 & 0.043 & 0.344 \\
\hline 10 & $\begin{array}{l}\text { (Log) Stem } \\
\text { height } \\
(\mathrm{cm})\end{array}$ & $\begin{array}{l}\text { Grazed (U, G) } \\
\text { Season } \\
\text { Creek distance } \\
\text { Latitude }\end{array}$ & $\begin{array}{l}14.87 \\
17.03 \\
14.25 \\
0.27\end{array}$ & $\begin{array}{l}1 \\
1 \\
1 \\
1\end{array}$ & $\begin{array}{l}<0.001 \\
<0.0001 \\
<0.001 \\
0.601\end{array}$ & $\begin{array}{l}-0.84 \pm .21 \\
-0.47 \pm .11 \\
-0.003 \pm .001 \\
-0.02 \pm .04\end{array}$ & $\begin{array}{r}-1.081 \pm .262 \\
-0.603 \pm .144 \\
-0.114 \pm .030 \\
-0.072 \pm .157\end{array}$ & 301 & 0.389 & 0.782 \\
\hline 11 & $\begin{array}{l}\text { (Log) Stem } \\
\text { density } \\
\text { (stems per } \\
0.09 \mathrm{~m}^{2} \text { ) }\end{array}$ & $\begin{array}{l}\text { Grazed }(\mathbf{U}, \mathbf{G}) \\
\text { Creek distance } \\
\text { Latitude }\end{array}$ & $\begin{array}{l}12.65 \\
3.64 \\
13.08\end{array}$ & $\begin{array}{l}1 \\
1 \\
1\end{array}$ & $\begin{array}{l}<0.001 \\
0.056 \\
<0.001\end{array}$ & $\begin{array}{l}0.60 \pm .15 \\
0.003 \pm .001 \\
0.19 \pm .04\end{array}$ & $\begin{array}{l}0.864 \pm .223 \\
0.128 \pm .068 \\
0.771 \pm .174\end{array}$ & 72 & 0.554 & 0.664 \\
\hline 12 & $\begin{array}{l}\text { (Log) Root } \\
\text { biomass } \\
0-15 \mathrm{~cm} \\
\text { (\% by mass) }\end{array}$ & $\begin{array}{l}\text { Grazed (U, G) } \\
\text { Season } \\
\text { Creek distance } \\
\text { Latitude }\end{array}$ & $\begin{array}{l}3.13 \\
0.90 \\
1.84 \\
14.62\end{array}$ & $\begin{array}{l}1 \\
1 \\
1 \\
1\end{array}$ & $\begin{array}{l}0.077 \\
0.343 \\
0.175 \\
<0.001\end{array}$ & $\begin{array}{l}-0.41 \pm .24 \\
0.12 \pm .13 \\
-0.001 \pm .001 \\
0.24 \pm .05\end{array}$ & $\begin{array}{l}-0.390 \pm .231 \\
0.113 \pm .128 \\
-0.040 \pm .030 \\
0.616 \pm .133\end{array}$ & 150 & 0.501 & 0.869 \\
\hline 13 & $\begin{array}{l}\text { (Log) } \\
\text { Periwinkle } \\
\text { density } \\
\text { (snails per } \\
0.09 \mathrm{~m}^{2} \text { ) }\end{array}$ & $\begin{array}{l}\text { Grazed }(U, G) \\
\text { Season } \\
\text { Creek distance } \\
\text { Latitude }\end{array}$ & $\begin{array}{l}0.14 \\
1.13 \\
17.70 \\
0.97\end{array}$ & $\begin{array}{l}1 \\
1 \\
1 \\
1\end{array}$ & $\begin{array}{l}0.712 \\
0.287 \\
<0.0001 \\
0.325\end{array}$ & $\begin{array}{l}0.08 \pm .26 \\
-0.19 \pm .19 \\
0.005 \pm .001 \\
-0.05 \pm .05\end{array}$ & $\begin{array}{l}0.087 \pm .274 \\
-0.195 \pm .197 \\
0.181 \pm .043 \\
-0.137 \pm .146\end{array}$ & 302 & 0.071 & 0.516 \\
\hline 14 & $\begin{array}{l}\text { (Log) Juvenile } \\
\text { fiddler crab } \\
\text { density } \\
\text { (burrows per } \\
0.09 \mathrm{~m}^{2} \text { ) }\end{array}$ & $\begin{array}{l}\text { Grazed }(U, G) \\
\text { Season } \\
\text { Creek distance } \\
\text { Latitude }\end{array}$ & $\begin{array}{l}0.02 \\
1.31 \\
1.50 \\
1.70\end{array}$ & $\begin{array}{l}1 \\
1 \\
1 \\
1\end{array}$ & $\begin{array}{l}0.887 \\
0.252 \\
0.221 \\
0.192\end{array}$ & $\begin{array}{l}0.04 \pm .31 \\
-0.29 \pm .21 \\
-0.002 \pm .001 \\
-0.08 \pm .07\end{array}$ & $\begin{array}{l}0.032 \pm .275 \\
-0.260 \pm .190 \\
-0.053 \pm \\
.042 \\
-0.209 \pm .183\end{array}$ & 302 & 0.079 & 0.525 \\
\hline 15 & $\begin{array}{l}\text { (Log) Adult } \\
\text { fiddler crab } \\
\text { density } \\
\text { (burrows per } \\
0.09 \mathrm{~m}^{2} \text { ) }\end{array}$ & $\begin{array}{l}\text { Grazed (U, G) } \\
\text { Season } \\
\text { Creek distance } \\
\text { Latitude }\end{array}$ & $\begin{array}{l}1.51 \\
1.02 \\
16.02 \\
20.08\end{array}$ & $\begin{array}{l}1 \\
1 \\
1 \\
1\end{array}$ & $\begin{array}{l}0.219 \\
0.313 \\
<0.0001 \\
<0.0001\end{array}$ & $\begin{array}{l}-0.19 \pm .17 \\
-0.17 \pm .15 \\
-0.004 \pm .001 \\
-0.19 \pm .03\end{array}$ & $\begin{array}{r}-0.217 \pm .185 \\
-0.189 \pm .164 \\
-0.161 \pm .040 \\
-0.603 \pm .102\end{array}$ & 302 & 0.416 & 0.586 \\
\hline 16 & $\begin{array}{l}\text { (Log) } \\
\text { Mud/sesarma } \\
\text { crab density } \\
\text { (burrows per } \\
0.09 \mathrm{~m}^{2} \text { ) }\end{array}$ & $\begin{array}{l}\text { Grazed }(U, G) \\
\text { Season } \\
\text { Creek distance } \\
\text { Latitude }\end{array}$ & $\begin{array}{l}0.28 \\
0.61 \\
13.51 \\
0.02\end{array}$ & $\begin{array}{l}1 \\
1 \\
1 \\
1\end{array}$ & $\begin{array}{l}0.595 \\
0.434 \\
<0.001 \\
0.899\end{array}$ & $\begin{array}{l}-0.06 \pm .11 \\
-0.08 \pm .09 \\
-0.002 \pm \\
.001 \\
-0.001 \pm .021\end{array}$ & $\begin{array}{r}-0.132 \pm .264 \\
-0.194 \pm .214 \\
-0.182 \pm .049 \\
-0.008 \pm .143\end{array}$ & 302 & 0.045 & 0.663 \\
\hline 17 & $\begin{array}{l}\text { (Log) Mussel } \\
\text { density } \\
\text { (mussels per } \\
\mathrm{m}^{2} \text { ) }\end{array}$ & $\begin{array}{l}\text { Grazed (U, G) } \\
\text { Season } \\
\text { Creek distance } \\
\text { Latitude }\end{array}$ & $\begin{array}{l}0.14 \\
0.45 \\
0.63 \\
0.23\end{array}$ & $\begin{array}{l}1 \\
1 \\
1 \\
1\end{array}$ & $\begin{array}{l}0.705 \\
0.501 \\
0.428 \\
0.635\end{array}$ & $\begin{array}{l}-0.10 \pm .28 \\
-0.14 \pm .24 \\
-0.001 \pm .002 \\
0.03 \pm .05\end{array}$ & $\begin{array}{l}-0.096 \pm .261 \\
-0.136 \pm .219 \\
-0.042 \pm .051 \\
0.066 \pm .142\end{array}$ & 303 & 0.010 & 0.347 \\
\hline
\end{tabular}


Table B3. Effect of grazing intensity on ecosystem properties in observational survey. Results of (generalised) linear mixed models to test the effect grazing intensity on multiple ecosystem properties. Pairwise P-values were calculated using Tukeys HSD test. In pairwise comparisons of ungrazed $(\mathrm{U})$, lightly $(\mathrm{L})$, moderately $(\mathrm{M})$ and heavily $(\mathrm{H})$ grazed marshes, ' $>$ ' and ' $<$ ' indicate that the direction of effect was significant, whereas ' $\geq$ ' and ' $\leq$ ' indicate that the direction of effect was not significant. Significant predictors and contrasts are highlighted in bold. All models were fitted with a random effect of marsh nested in site. Marginal (Marg) $R^{2}$ values relate to fixed effects only, Conditional (Cond) $R^{2}$ values relate to fixed + random effects.

\begin{tabular}{|c|c|c|c|c|c|c|c|c|c|c|}
\hline \multirow{2}{*}{$\begin{array}{l}\bar{\Phi} \\
\overline{0} \\
\Sigma\end{array}$} & \multirow[t]{2}{*}{ Response } & \multirow[t]{2}{*}{ Fixed effects } & \multirow[t]{2}{*}{$\chi^{2}$} & \multirow[t]{2}{*}{ df } & \multirow[t]{2}{*}{$\mathbf{P}$} & \multirow[t]{2}{*}{$\mathbf{n}$} & \multirow{2}{*}{$\begin{array}{l}\text { Marg } \\
\mathbf{R}^{2}\end{array}$} & \multirow{2}{*}{$\begin{array}{l}\text { Cond } \\
R^{2}\end{array}$} & \multicolumn{2}{|c|}{ Pairwise contrasts } \\
\hline & & & & & & & & & Effect & $\mathbf{P}$ \\
\hline \multirow[t]{3}{*}{1} & Salinity & Grazing intensity & 0.45 & 3 & 0.930 & 71 & 0.060 & 0.568 & & \\
\hline & & Creek distance & 1.58 & 1 & 0.208 & & & & & \\
\hline & & Latitude & 1.03 & 1 & 0.311 & & & & & \\
\hline \multirow[t]{3}{*}{2} & $\mathrm{pH}$ & Grazing intensity & 1.92 & 3 & 0.590 & 71 & 0.192 & 0.384 & & \\
\hline & & Creek distance & 0.22 & 1 & 0.636 & & & & & \\
\hline & & Latitude & 3.63 & 1 & 0.057 & & & & & \\
\hline \multirow[t]{4}{*}{3} & (Log) Redox & Grazing intensity & 0.67 & 3 & 0.879 & 141 & 0.295 & 0.656 & & \\
\hline & $(+400$ to make & Season & 12.25 & 1 & $<.001$ & & & & & \\
\hline & positive) & Creek distance & 0.25 & 1 & 0.619 & & & & & \\
\hline & & Latitude & 0.33 & 1 & 0.564 & & & & & \\
\hline \multirow[t]{4}{*}{4} & (Log) Soil organic & Grazing intensity & 7.14 & 3 & 0.068 & 150 & 0.269 & 0.814 & & \\
\hline & carbon & Season & 0.05 & 1 & 0.827 & & & & & \\
\hline & $0-15 \mathrm{~cm}$ & Creek distance & 6.92 & 1 & 0.009 & & & & & \\
\hline & (\% by mass) & Latitude & 4.28 & 1 & 0.039 & & & & & \\
\hline \multirow[t]{4}{*}{$4 . i$} & (Log) Soil organic & Grazing intensity & 2.92 & 3 & 0.404 & 150 & 0.203 & 0.752 & & \\
\hline & carbon & Season & 0.09 & 1 & 0.768 & & & & & \\
\hline & $0-5 \mathrm{~cm}$ & Creek distance & 1.02 & 1 & 0.313 & & & & & \\
\hline & (\% by mass) & Latitude & 3.38 & 1 & 0.066 & & & & & \\
\hline \multirow[t]{6}{*}{ 4.ii } & (Log) Soil organic & Grazing intensity & 8.09 & 3 & 0.044 & 150 & 0.207 & 0.822 & $U \geq L$ & 0.329 \\
\hline & carbon & Season & 0.09 & 1 & 0.768 & & & & $U \geq M$ & 0.213 \\
\hline & $5-15 \mathrm{~cm}$ & Creek distance & 13.62 & 1 & $<.001$ & & & & $\mathrm{U} \geq \mathrm{H}$ & 0.618 \\
\hline & (\% by mass) & Latitude & 1.33 & 1 & 0.250 & & & & $L \geq M$ & 0.999 \\
\hline & & & & & & & & & $\mathrm{L} \leq \mathrm{H}$ & 0.976 \\
\hline & & & & & & & & & $\mathrm{M} \leq \mathrm{H}$ & 0.798 \\
\hline \multirow[t]{4}{*}{5} & Sand content in & Grazing intensity & 7.24 & 3 & 0.065 & 82 & 0.282 & 0.712 & & \\
\hline & soil & Season & 1.72 & 1 & 0.190 & & & & & \\
\hline & (\%) & Creek distance & 6.01 & 1 & 0.014 & & & & & \\
\hline & & Latitude & 2.70 & 1 & 0.100 & & & & & \\
\hline \multirow[t]{4}{*}{6} & Clay content in & Grazing intensity & 6.06 & 3 & 0.109 & 82 & 0.277 & 0.768 & & \\
\hline & soil & Season & 5.97 & 1 & 0.015 & & & & & \\
\hline & (\%) & Creek distance & 3.55 & 1 & 0.060 & & & & & \\
\hline & & Latitude & 1.91 & 1 & 0.167 & & & & & \\
\hline \multirow[t]{4}{*}{7} & Living veg cover & Grazing intensity & 6.71 & 3 & 0.082 & 300 & 0.231 & 0.671 & & \\
\hline & $(\%)$ & Season & 0.97 & 1 & 0.326 & & & & & \\
\hline & & Creek distance & 6.04 & 1 & 0.014 & & & & & \\
\hline & & Latitude & 2.98 & 1 & 0.084 & & & & & \\
\hline \multirow[t]{4}{*}{8} & Veg richness & Grazing intensity & 2.39 & 3 & 0.495 & 308 & 0.031 & 0.282 & & \\
\hline & & Season & 0.02 & 1 & 0.886 & & & & & \\
\hline & & Creek distance & 0.37 & 1 & 0.546 & & & & & \\
\hline & & Latitude & 0.80 & 1 & 0.371 & & & & & \\
\hline \multirow[t]{4}{*}{9} & Spartina cover & Grazing intensity & 2.34 & 3 & 0.506 & 306 & 0.047 & 0.346 & & \\
\hline & (Binomial model: & Season & 0.04 & 1 & 0.845 & & & & & \\
\hline & $100 \%$, not $100 \%)$ & Creek distance & 1.33 & 1 & 0.249 & & & & & \\
\hline & & Latitude & 0.57 & 1 & 0.451 & & & & & \\
\hline
\end{tabular}


Table B3 cont.

\begin{tabular}{|c|c|c|c|c|c|c|c|c|c|c|}
\hline \multirow{2}{*}{$\frac{\bar{d}}{\stackrel{0}{0}}$} & \multirow[t]{2}{*}{ Response } & \multirow[t]{2}{*}{ Fixed effects } & \multirow[t]{2}{*}{$\chi^{2}$} & \multirow[t]{2}{*}{ df } & \multirow[t]{2}{*}{$\mathbf{P}$} & \multirow[t]{2}{*}{$\mathbf{n}$} & \multirow{2}{*}{$\begin{array}{l}\text { Marg } \\
\mathbf{R}^{2}\end{array}$} & \multirow{2}{*}{$\begin{array}{l}\text { Cond } \\
\mathbf{R}^{2}\end{array}$} & \multicolumn{2}{|c|}{ Pairwise contrasts } \\
\hline & & & & & & & & & Effect & $\mathbf{P}$ \\
\hline \multirow[t]{6}{*}{10} & (Log) Stem height & Grazing intensity & 19.3 & 3 & $<.001$ & 301 & 0.389 & 0.782 & $U \geq L$ & 0.081 \\
\hline & $(\mathrm{cm})$ & Season & 1 & 1 & 0.056 & & & & $U>M$ & 0.001 \\
\hline & & Creek distance & 3.66 & 1 & $<.001$ & & & & $U>H$ & $<0.001$ \\
\hline & & Latitude & 13.2 & 1 & 0.237 & & & & $L \geq M$ & 0.461 \\
\hline & & & 7 & & & & & & $\mathrm{~L} \geq \mathrm{H}$ & 0.133 \\
\hline & & & 1.40 & & & & & & $\mathrm{M} \geq \mathrm{H}$ & 0.543 \\
\hline \multirow[t]{6}{*}{11} & (Log) Stem & Grazing intensity & 14.8 & 3 & 0.002 & 72 & 0.595 & 0.711 & $U \leq L$ & 0.992 \\
\hline & density & Creek distance & 3.33 & 1 & 0.068 & & & & $\mathrm{U}<\mathrm{M}$ & 0.041 \\
\hline & (stems per & Latitude & 7.72 & 1 & 0.005 & & & & $\mathrm{U} \leq \mathrm{H}$ & 0.052 \\
\hline & $\left.0.09 m^{2}\right)$ & & & & & & & & $L \leq M$ & 0.353 \\
\hline & & & & & & & & & $\mathrm{L}<\mathrm{H}$ & 0.238 \\
\hline & & & & & & & & & $\mathrm{M} \leq \mathrm{H}$ & 0.621 \\
\hline \multirow[t]{4}{*}{12} & (Log) Root & Grazing intensity & 3.81 & 3 & 0.282 & 150 & 0.502 & 0.867 & & \\
\hline & biomass & Season & 1.35 & 1 & 0.246 & & & & & \\
\hline & $0-15 \mathrm{~cm}$ & Creek distance & 1.84 & 1 & 0.175 & & & & & \\
\hline & (\% by mass) & Latitude & 14.6 & 1 & $<.001$ & & & & & \\
\hline \multirow[t]{4}{*}{13} & (Log) Periwinkle & Grazing intensity & 0.28 & 3 & 0.965 & 302 & 0.072 & 0.532 & & \\
\hline & density & Season & 0.84 & 1 & 0.361 & & & & & \\
\hline & (snails per & Creek distance & 17.6 & 1 & $<.0001$ & & & & & \\
\hline & $\left.0.09 \mathrm{~m}^{2}\right)$ & Latitude & 0.69 & 1 & 0.406 & & & & & \\
\hline \multirow[t]{4}{*}{14} & (Log) Juvenile & Grazing intensity & 1.39 & 3 & 0.708 & 302 & 0.086 & 0.539 & & \\
\hline & fiddler crab & Season & 1.10 & 1 & 0.294 & & & & & \\
\hline & density & Creek distance & 1.40 & 1 & 0.237 & & & & & \\
\hline & $\begin{array}{l}\text { (burrows per } \\
0.09 \mathrm{~m}^{2} \text { ) }\end{array}$ & Latitude & 1.91 & 1 & 0.168 & & & & & \\
\hline \multirow[t]{4}{*}{15} & (Log) Adult fiddler & Grazing intensity & 6.37 & 3 & 0.095 & 302 & 0.436 & 0.574 & & \\
\hline & crab density & Season & 0.15 & 1 & 0.697 & & & & & \\
\hline & (burrows per & Creek distance & 14.5 & 1 & $<.001$ & & & & & \\
\hline & $\left.0.09 m^{2}\right)$ & Latitude & 23.4 & 1 & $<.0001$ & & & & & \\
\hline \multirow[t]{4}{*}{16} & (Log) & Grazing intensity & 6.64 & 3 & 0.084 & 302 & 0.137 & 0.424 & & \\
\hline & Mud/sesarma & Season & 0.21 & 1 & 0.643 & & & & & \\
\hline & crab density & Creek distance & 13.2 & 1 & $<.001$ & & & & & \\
\hline & $\begin{array}{l}\text { (burrows per } \\
0.09 \mathrm{~m}^{2} \text { ) }\end{array}$ & Latitude & 1.20 & 1 & 0.274 & & & & & \\
\hline \multirow[t]{4}{*}{17} & (Log) Mussel & Grazing intensity & 0.21 & 3 & 0.975 & 303 & 0.011 & 0.366 & & \\
\hline & density & Season & 0.48 & 1 & 0.488 & & & & & \\
\hline & (mussels per $\mathrm{m}^{2}$ ) & Creek distance & 0.63 & 1 & 0.427 & & & & & \\
\hline & & Latitude & 0.28 & 1 & 0.597 & & & & & \\
\hline
\end{tabular}


Table B4. Baseline comparisons, control vs experimental plots. Results of linear (mixed) models to test whether control (grazed) and experimental (ungrazed) plots differed at $\mathrm{T}=0$. Significant predictors are highlighted in bold. Where treatment was found to be significant, I have shown the coefficient (coef) of the effect of treatment relative to a baseline value of control grazed.

\begin{tabular}{|c|c|c|c|c|c|c|c|c|}
\hline $\begin{array}{l}\bar{\Phi} \\
\frac{0}{2} \\
\Sigma\end{array}$ & Response & $T$ & Predictors & $\mathbf{F}$ & df & $\mathbf{P}$ & $\mathrm{n}$ & Coef \pm S.E. \\
\hline \multirow[t]{2}{*}{$\mathrm{i}$} & Elevation $(\mathrm{cm})$ & 0 & Treatment (grazed, ungrazed) & 0.815 & 1 & 0.397 & 16 & \\
\hline & & & Pair (8 pairs) & 2.998 & 7 & 0.085 & & \\
\hline \multirow[t]{2}{*}{ ii } & Salinity (ppt) & 0 & Treatment (grazed, ungrazed) & 1.750 & 1 & 0.227 & 16 & \\
\hline & & & Pair (8 pairs) & 8.234 & 7 & 0.006 & & \\
\hline \multirow[t]{2}{*}{ iii } & $\mathrm{pH}$ & 0 & Treatment (grazed, ungrazed) & 0.452 & 1 & 0.523 & 16 & \\
\hline & & & Pair (8 pairs) & 5.743 & 7 & 0.017 & & \\
\hline \multirow[t]{2}{*}{ iv } & (Log) Soil organic & 0 & Treatment (grazed, ungrazed) & 1.187 & 1 & 0.312 & 16 & \\
\hline & carbon $(\%)$ & & Pair (8 pairs) & 47.829 & 7 & $<.0001$ & & \\
\hline \multirow[t]{2}{*}{$\mathrm{v}$} & Proportion of & 0 & Treatment (grazed, ungrazed) & 1.595 & 1 & 0.247 & 16 & \\
\hline & Spartina & & Pair (8 pairs) & 12.795 & 7 & 0.002 & & \\
\hline \multirow[t]{2}{*}{ vi } & Proportion of & 0 & Treatment (grazed, ungrazed) & 3.755 & 1 & 0.094 & 16 & \\
\hline & Salicornia & & Pair (8 pairs) & 15.146 & 7 & 0.001 & & \\
\hline \multirow[t]{2}{*}{ vii } & Proportion of & 0 & Treatment (grazed, ungrazed) & 1.000 & 1 & 0.351 & 16 & \\
\hline & Distichlis & & Pair (8 pairs) & 9.143 & 7 & 0.005 & & \\
\hline \multirow[t]{2}{*}{ viii } & Stem height $(\mathrm{cm})$ & 0 & Treatment (grazed, ungrazed) & 6.503 & 1 & 0.038 & 16 & $-1.25 \pm 0.49$ \\
\hline & & & Pair (8 pairs) & 33.214 & 7 & $<.0001$ & & \\
\hline \multirow[t]{2}{*}{ ix } & Stem density & 0 & Treatment (grazed, ungrazed) & 0.026 & 1 & 0.875 & 16 & \\
\hline & & & Pair (8 pairs) & 1.706 & 7 & 0.249 & & \\
\hline \multirow[t]{2}{*}{$\mathrm{x}$} & (Log) Root biomass & 0 & Treatment (grazed, ungrazed) & 1.285 & 1 & 0.294 & 16 & \\
\hline & (\%) & & Pair (8 pairs) & 18.176 & 7 & 0.001 & & \\
\hline \multirow[t]{2}{*}{$x i$} & Periwinkle snail & 0 & Treatment (grazed, ungrazed) & 0.668 & 1 & 0.441 & 16 & \\
\hline & density & & Pair (8 pairs) & 7.908 & 7 & 0.007 & & \\
\hline \multirow[t]{2}{*}{ xii } & Juvenile fiddler & 0 & Treatment (grazed, ungrazed) & 0.046 & 1 & 0.836 & 16 & \\
\hline & crab density & & Pair (8 pairs) & 7.917 & 7 & 0.007 & & \\
\hline \multirow[t]{2}{*}{ xiii } & Adult fiddler crab & 0 & Treatment (grazed, ungrazed) & 1.939 & 1 & 0.206 & 16 & \\
\hline & density & & Pair (8 pairs) & 0.943 & 7 & 0.530 & & \\
\hline \multirow[t]{2}{*}{ xiv } & Large crab density & 0 & Treatment (grazed, ungrazed) & 0.180 & 1 & 0.685 & 16 & \\
\hline & & & Pair (8 pairs) & 2.231 & 7 & 0.156 & & \\
\hline \multirow[t]{2}{*}{$x v$} & Mussel mound & 0 & Treatment (grazed, ungrazed) & 0.368 & 1 & 0.536 & 16 & \\
\hline & density & & Pair (8 pairs) & 9.053 & 7 & 0.005 & & \\
\hline \multirow[t]{2}{*}{$x v i$} & Hoofprint density & 0 & Treatment (grazed, ungrazed) & 0.059 & 1 & 0.815 & 16 & \\
\hline & & & Pair (8 pairs) & 7.656 & 8 & 0.008 & & \\
\hline
\end{tabular}


Table B5. Baseline comparisons, control vs reference points. Results of linear (mixed) models to test whether control (grazed) plots and unmarked reference points (grazed ref) differed at $\mathrm{T}=0$ and $\mathrm{T}=7 / 18$ months. For models that combined data from $\mathrm{T}=7$ and $\mathrm{T}=18$ months, I used plot as a random effect to control for repeated measures. Significant predictors are highlighted in bold. Where treatment was found to be significant, I have shown the coefficient (coef) of the effect of treatment relative to a baseline value of control grazed.

\begin{tabular}{|c|c|c|c|c|c|c|c|c|}
\hline $\begin{array}{l}\bar{\Phi} \\
\bar{\delta} \\
\Sigma\end{array}$ & Response & $\mathbf{T}$ & Predictors & $\mathbf{F}$ & df & $\mathbf{P}$ & n & Coef \pm SE \\
\hline \multirow[t]{2}{*}{1} & Elevation $(\mathrm{cm})$ & 0 & Treatment (grazed, grazed ref) & 0.000 & 1 & 0.989 & 14 & \\
\hline & & & Site (DM, RK) & 0.169 & 1 & 0.689 & & \\
\hline \multirow[t]{2}{*}{ II } & Living plant cover & 18 & Treatment (grazed, grazed ref) & 1.000 & 1 & 0.335 & 38 & \\
\hline & $(\%)$ & & Site (DM, RK) & 0.042 & 1 & 0.843 & & \\
\hline \multirow[t]{2}{*}{ IIIa } & Dead plant cover & 0 & Treatment (grazed, grazed ref) & 0.224 & 1 & 0.645 & 14 & \\
\hline & $(\%)$ & & Site (DM, RK) & 2.045 & 1 & 0.181 & & \\
\hline \multirow[t]{2}{*}{ IIIb } & Dead plant cover & 18 & Treatment (grazed, grazed ref) & 0.010 & 1 & 0.924 & 38 & \\
\hline & $(\%)$ & & Site (DM, RK) & 0.217 & 1 & 0.650 & & \\
\hline \multirow[t]{2}{*}{ IVa } & Stem height $(\mathrm{cm})$ & 0 & Treatment (grazed, grazed ref) & 0.061 & 1 & 0.810 & 14 & \\
\hline & & & Site (DM, RK) & 2.862 & 1 & 0.120 & & \\
\hline \multirow[t]{2}{*}{$\mathrm{IVb}$} & Stem height $(\mathrm{cm})$ & $7+18$ & Treatment (grazed, grazed ref) & 5.435 & 1 & 0.031 & 52 & $+5.73 \pm 2.46$ \\
\hline & & & Site (DM, RK) & 5.116 & 1 & 0.043 & & \\
\hline \multirow[t]{2}{*}{ IVc } & Stem height (cm) & $7+18$ & Treatment (grazed, grazed ref) & 3.977 & 1 & 0.064 & 42 & \\
\hline & (excluding die-off) & & Site (DM, RK) & 14.977 & 1 & 0.002 & & \\
\hline \multirow[t]{2}{*}{ Va } & Stem density & 0 & Treatment (grazed, grazed ref) & 0.138 & 1 & 0.718 & 14 & \\
\hline & & & Site (DM, RK) & 19.297 & 1 & 0.001 & & \\
\hline \multirow[t]{2}{*}{$\mathrm{Vb}$} & Stem density & $7+18$ & Treatment (grazed, grazed ref) & 0.781 & 1 & 0.394 & 52 & \\
\hline & & & Site (DM, RK) & 0.478 & 1 & 0.503 & & \\
\hline \multirow[t]{2}{*}{ VI } & Proportion of & $7+18$ & Treatment (grazed, grazed ref) & 1.934 & 1 & 0.171 & 48 & \\
\hline & stems bitten & & Site (DM, RK) & 1.636 & 1 & 0.208 & & \\
\hline \multirow[t]{2}{*}{ VIIa } & Hoofprint density & 0 & Treatment (grazed, grazed ref) & 1.040 & 1 & 0.330 & 14 & \\
\hline & & & Site (DM, RK) & 0.462 & 1 & 0.511 & & \\
\hline \multirow[t]{2}{*}{ VIIb } & (Log) Hoofprint & $7+18$ & Treatment (grazed, grazed ref) & 2.190 & 1 & 0.161 & 52 & \\
\hline & density & & Site (DM, RK) & 10.106 & 1 & 0.010 & & \\
\hline
\end{tabular}


Table B6. Effect of grazing on ecosystem properties after 18 months. Results of linear mixed models to test whether control (grazed) and experimental (ungrazed) plots differed at $T=18$. Significant predictors are highlighted in bold. FDR-P shows $\mathrm{P}$-values that have been adjusted for multiple comparisons using the false discovery rate (Benjamini and Hochberg 1995). Coefficients are for the effect of removing grazers relative to the grazed control. We give coefficients (coef) and standard errors (SE) both for the original modelled scale and for models using standardised Z-transformed data (Z-coef). Marginal (Marg) $R^{2}$ values relate to fixed effects only, Conditional (Cond) $R^{2}$ values relate to fixed + random effects. All models were fitted with the fixed effect of treatment (grazed, ungrazed) and a random effect of pair. All models had $n=48$ and $d f=1,39$.

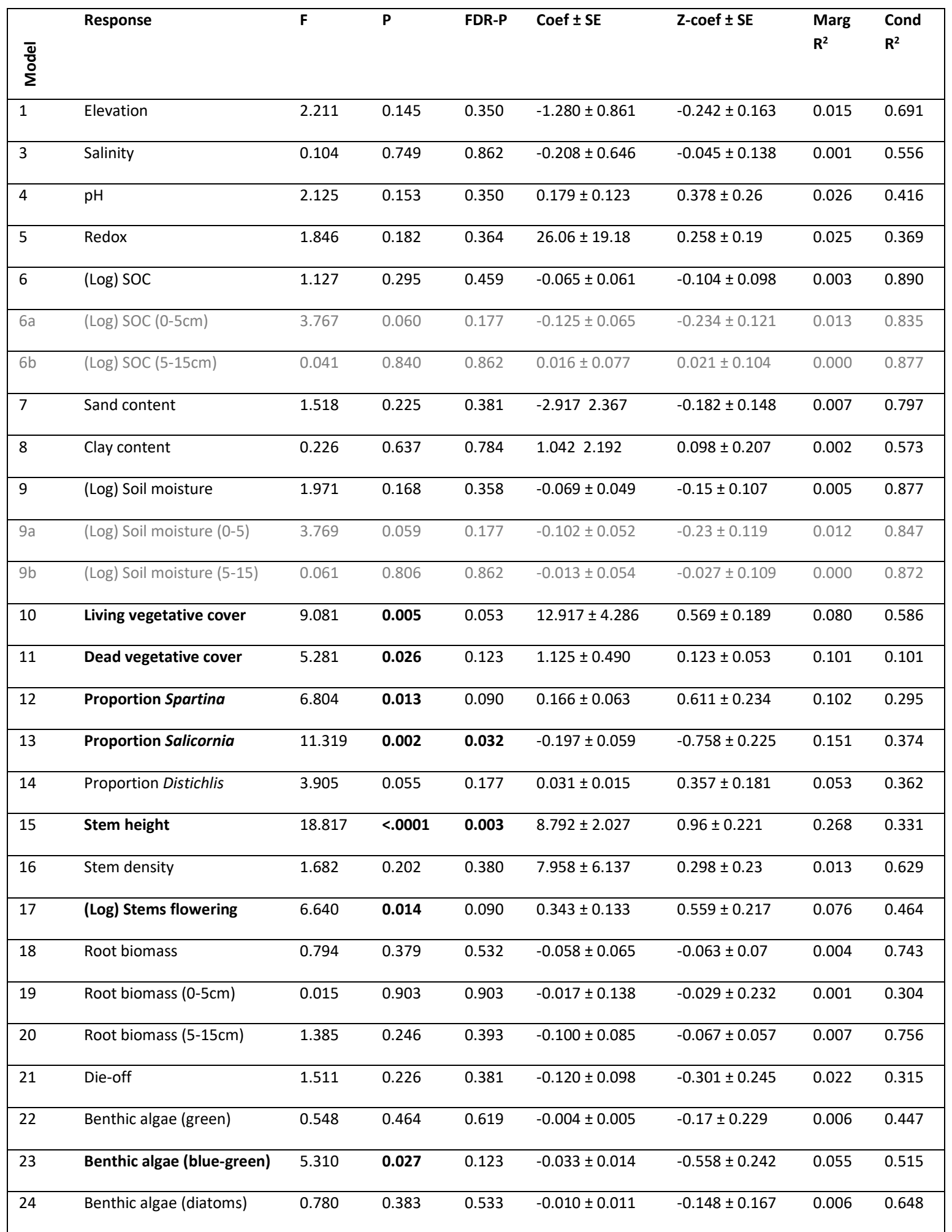


Appendix B

\begin{tabular}{|lllllllll|}
\hline 25 & (Log) Periwinkle density & 0.437 & 0.512 & 0.655 & $-0.122 \pm 0.185$ & $-0.12 \pm 0.182$ & 0.003 & 0.633 \\
\hline 26 & $\begin{array}{l}\text { Juvenile fiddler crab } \\
\text { density }\end{array}$ & 2.686 & 0.109 & 0.291 & $-4.375 \pm 2.670$ & $-0.406 \pm 0.248$ & 0.035 & 0.380 \\
\hline 27 & $\begin{array}{l}\text { (Log) Adult fiddler crab } \\
\text { density }\end{array}$ & 4.582 & $\mathbf{0 . 0 3 9}$ & 0.156 & $0.505 \pm 0.236$ & $0.552 \pm 0.258$ & 0.075 & 0.228 \\
\hline 28 & Marsh/mud crab density & 0.044 & 0.835 & 0.862 & $-0.042 \pm 0.199$ & $-0.044 \pm 0.212$ & 0.001 & 0.237 \\
\hline 29 & (Log) Mussel density & 0.059 & 0.809 & 0.862 & $-0.080 \pm 0.328$ & $-0.059 \pm 0.241$ & 0.001 & 0.338 \\
\hline
\end{tabular}


Table B7. Effect of grazing on ecosystem properties at each time point. Results of linear mixed models to test whether control (grazed, G) and experimental (ungrazed, U) plots differed at each time point. In pairwise comparisons, ' $>$ ' and ' $<$ ' indicate that the response was significantly higher or lower in grazed plots, whereas ' $\geq$ ' and ' $\leq$ ' show the response was higher or lower but not significantly different. Pairwise P-values were calculated using Tukeys HSD test. Significant predictors and contrasts are highlighted in bold. All models were fitted with a random effect of pair.

\begin{tabular}{|c|c|c|c|c|c|c|c|c|c|}
\hline \multirow{2}{*}{$\begin{array}{l}\bar{d} \\
\bar{\delta}\end{array}$} & \multirow[t]{2}{*}{ Response } & \multirow[t]{2}{*}{ Fixed effect } & \multirow[t]{2}{*}{$\mathbf{F}$} & \multirow[t]{2}{*}{ df } & \multirow[t]{2}{*}{$\mathbf{P}$} & \multirow[t]{2}{*}{$\mathbf{n}$} & \multicolumn{3}{|c|}{ Pairwise contrasts } \\
\hline & & & & & & & Time & Effect & $\mathbf{P}$ \\
\hline \multirow[t]{2}{*}{1} & Living vegetative & Treatment $\mathrm{x}$ & 31.81 & 3,85 & $<0.0001$ & 96 & 13 & $\mathbf{G}<\mathbf{U}$ & $<0.0001$ \\
\hline & cover & Time & & & & & 18 & $\mathbf{G}<\mathbf{U}$ & $<0.001$ \\
\hline \multirow[t]{3}{*}{2} & Dead vegetative & Treatment $\mathrm{x}$ & 22.38 & 5,99 & $<0.0001$ & 112 & 0 & $\mathrm{G} \geq \mathrm{U}$ & 0.316 \\
\hline & cover & Time & & & & & 13 & $\mathbf{G}>\mathbf{U}$ & $<0.0001$ \\
\hline & & & & & & & 18 & $\mathrm{G} \geq \mathrm{U}$ & 0.562 \\
\hline \multirow[t]{4}{*}{3} & Proportion & Treatment $\mathrm{x}$ & 3.59 & 7,113 & 0.002 & 128 & 0 & $\mathrm{G} \leq \mathrm{U}$ & 0.711 \\
\hline & Spartina cover & Time & & & & & 7 & $\mathrm{G} \leq \mathrm{U}$ & 0.711 \\
\hline & & & & & & & 13 & $\mathbf{G}<\mathbf{U}$ & 0.001 \\
\hline & & & & & & & 18 & $\mathbf{G}<\mathbf{U}$ & 0.007 \\
\hline \multirow[t]{4}{*}{4} & Proportion & Treatment $\mathbf{x}$ & 4.93 & 7,113 & $<0.0001$ & 128 & 0 & $\mathrm{G} \geq \mathrm{U}$ & 0.599 \\
\hline & Salicornia cover & Time & & & & & 7 & $G \geq U$ & 0.529 \\
\hline & & & & & & & 13 & $\mathbf{G}>\mathbf{U}$ & $<0.001$ \\
\hline & & & & & & & 18 & $\mathbf{G}>\mathbf{U}$ & $<0.001$ \\
\hline \multirow[t]{4}{*}{5} & Stem height & Treatment $\mathrm{x}$ & 15.39 & 7,113 & $<0.0001$ & 128 & 0 & $G \geq U$ & 0.694 \\
\hline & & Time & & & & & 7 & $\mathbf{G}<\mathbf{U}$ & 0.002 \\
\hline & & & & & & & 13 & $\mathbf{G}<\mathbf{U}$ & $<0.001$ \\
\hline & & & & & & & 18 & $\mathbf{G}<\mathbf{U}$ & $<0.0001$ \\
\hline \multirow[t]{2}{*}{6} & (Log) Stems & Treatment $\mathrm{x}$ & 7.88 & 3,53 & $<0.001$ & 64 & 7 & $\mathbf{G}<\mathbf{U}$ & 0.006 \\
\hline & flowering & Time & & & & & 18 & $\mathrm{G} \leq \mathrm{U}$ & 0.058 \\
\hline \multirow[t]{2}{*}{7} & Benthic algae & Treatment $\mathrm{x}$ & 25.03 & 3,85 & $<0.0001$ & 96 & 13 & $G \geq U$ & 0.408 \\
\hline & (blue-green) & Time & & & & & 18 & $\mathbf{G}>\mathbf{U}$ & 0.008 \\
\hline \multirow[t]{4}{*}{8} & (Log) Adult fiddler & Treatment $\mathrm{x}$ & 7.23 & 7,113 & $<0.0001$ & 128 & 0 & $\mathrm{G} \geq \mathrm{U}$ & 0.373 \\
\hline & crab density & Time & & & & & 7 & $\mathrm{G} \leq \mathrm{U}$ & 0.765 \\
\hline & & & & & & & 13 & $\mathbf{G}<\mathbf{U}$ & $<0.0001$ \\
\hline & & & & & & & 18 & $\mathbf{G}<\mathbf{U}$ & 0.013 \\
\hline
\end{tabular}


Table B8. Effect of grazing on recovery from damage. Results of linear mixed models to test the effect of an interaction between grazing treatment and simulated hog damage, 6 months and 12 months after damage. Pairwise P-values were calculated using Tukeys HSD test. Significant predictors are highlighted in bold. The contrast between grazed damaged (GD) and ungrazed damaged (UD) relates to 'absolute' (across-grazing) recovery. The contrasts between grazed control (GC) and grazed damaged (GD), and between ungrazed control (UC) and ungrazed damaged (UD) relate to 'relative' (within-grazing) recovery. In pairwise comparisons, ' $>$ ' and ' $<$ ' indicate that the direction of effect was significant, whereas ' $\geq$ ' and ' $\leq$ ' indicate that the direction of effect was not significant. All models were fitted with a random effect of pair. Marginal (Marg) $R^{2}$ values relate to fixed effects only, Conditional (Cond) $\mathrm{R}^{2}$ values relate to fixed + random effects. $\mathrm{N}=64$ for all models.

\begin{tabular}{|c|c|c|c|c|c|c|c|c|c|}
\hline \multirow{2}{*}{$\frac{\bar{d}}{\frac{0}{2}}$} & \multirow[t]{2}{*}{ Response } & \multirow[t]{2}{*}{ Time } & \multicolumn{3}{|c|}{ Treatment x Damage } & \multicolumn{2}{|c|}{$\begin{array}{l}\text { Pairwise contrasts of } \\
\text { factor levels }\end{array}$} & \multirow[t]{2}{*}{$\begin{array}{l}\text { Marg } \\
\mathbf{R}^{\mathbf{2}}\end{array}$} & \multirow[t]{2}{*}{$\begin{array}{l}\text { Cond } \\
\mathbf{R}^{2}\end{array}$} \\
\hline & & & $\mathbf{F}$ & df & $\mathbf{P}$ & Effect & $\mathbf{P}$ & & \\
\hline \multirow[t]{4}{*}{$1 \mathrm{a}$} & Living plant cover & 6 & 26.21 & 3,53 & $<0.0001$ & $\mathrm{GC}<\mathrm{UC}$ & $<0.0001$ & 0.361 & 0.703 \\
\hline & $(\%)$ & & & & & $\mathrm{GD} \leq \mathrm{UD}$ & 0.052 & & \\
\hline & & & & & & GC $>$ GD & $<0.001$ & & \\
\hline & & & & & & UC > UD & $<0.0001$ & & \\
\hline \multirow[t]{4}{*}{$1 b$} & Living plant cover & 12 & 13.22 & 3,53 & $<0.0001$ & $\mathbf{G C}<\mathrm{UC}$ & 0.006 & 0.256 & 0.594 \\
\hline & $(\%)$ & & & & & $\mathrm{GD} \leq \mathrm{UD}$ & 0.688 & & \\
\hline & & & & & & GC $>$ GD & 0.003 & & \\
\hline & & & & & & $U C>$ UD & $<0.0001$ & & \\
\hline \multirow[t]{4}{*}{$2 a$} & Dead plant cover & 6 & 28.23 & 3,53 & $<0.0001$ & $\mathrm{GC}<\mathrm{UC}$ & $<0.0001$ & 0.414 & 0.692 \\
\hline & $(\%)$ & & & & & $\mathrm{GD} \leq \mathrm{UD}$ & 0.767 & & \\
\hline & & & & & & $\mathrm{GC} \geq \mathrm{GD}$ & 0.157 & & \\
\hline & & & & & & $U C>$ UD & $<0.0001$ & & \\
\hline \multirow[t]{4}{*}{$2 b$} & Dead plant cover & 12 & 14.56 & 3,53 & 0.001 & $\mathrm{GC}<\mathrm{UC}$ & 0.014 & 0.222 & 0.251 \\
\hline & $(\%)$ & & & & & $\mathrm{GD} \leq \mathrm{UD}$ & 0.331 & & \\
\hline & & & & & & GC $>$ GD & 0.043 & & \\
\hline & & & & & & $U C>$ UD & 0.010 & & \\
\hline \multirow[t]{4}{*}{$3 a$} & Stem height & 6 & 13.03 & 3,53 & $<0.0001$ & $\mathbf{G C}<\mathrm{UC}$ & 0.0001 & 0.312 & 0.498 \\
\hline & $(\mathrm{cm})$ & & & & & $\mathrm{GD} \leq \mathrm{UD}$ & 0.332 & & \\
\hline & & & & & & GC $>$ GD & 0.021 & & \\
\hline & & & & & & UC > UD & 0.0001 & & \\
\hline \multirow[t]{4}{*}{$3 b$} & Stem height & 12 & 8.67 & 3,53 & $<0.0001$ & $\mathrm{GC}<\mathrm{UC}$ & $<0.0001$ & 0.269 & 0.347 \\
\hline & $(\mathrm{cm})$ & & & & & $\mathrm{GD} \leq \mathrm{UD}$ & 0.308 & & \\
\hline & & & & & & $\mathrm{GC} \geq \mathrm{GD}$ & 0.927 & & \\
\hline & & & & & & $U C>$ UD & 0.034 & & \\
\hline \multirow[t]{4}{*}{$4 a$} & Stem density & 6 & 9.52 & 3,53 & $<0.0001$ & $\mathbf{G C}<\mathrm{UC}$ & 0.038 & 0.231 & 0.497 \\
\hline & (stems per $0.09 \mathrm{~m}^{2}$ ) & & & & & $\mathrm{GD} \leq \mathrm{UD}$ & 0.104 & & \\
\hline & & & & & & GC $>$ GD & $<0.001$ & & \\
\hline & & & & & & UC $>$ UD & 0.004 & & \\
\hline \multirow[t]{4}{*}{$4 b$} & Stem density & 12 & 3.04 & 3,53 & 0.037 & $\mathrm{GC} \leq \mathrm{UC}$ & 0.224 & 0.066 & 0.541 \\
\hline & (stems per $0.09 \mathrm{~m}^{2}$ ) & & & & & $\mathrm{GD} \leq \mathrm{UD}$ & 0.625 & & \\
\hline & & & & & & $\mathrm{GC} \geq \mathrm{GD}$ & 0.080 & & \\
\hline & & & & & & UC > UD & 0.045 & & \\
\hline
\end{tabular}




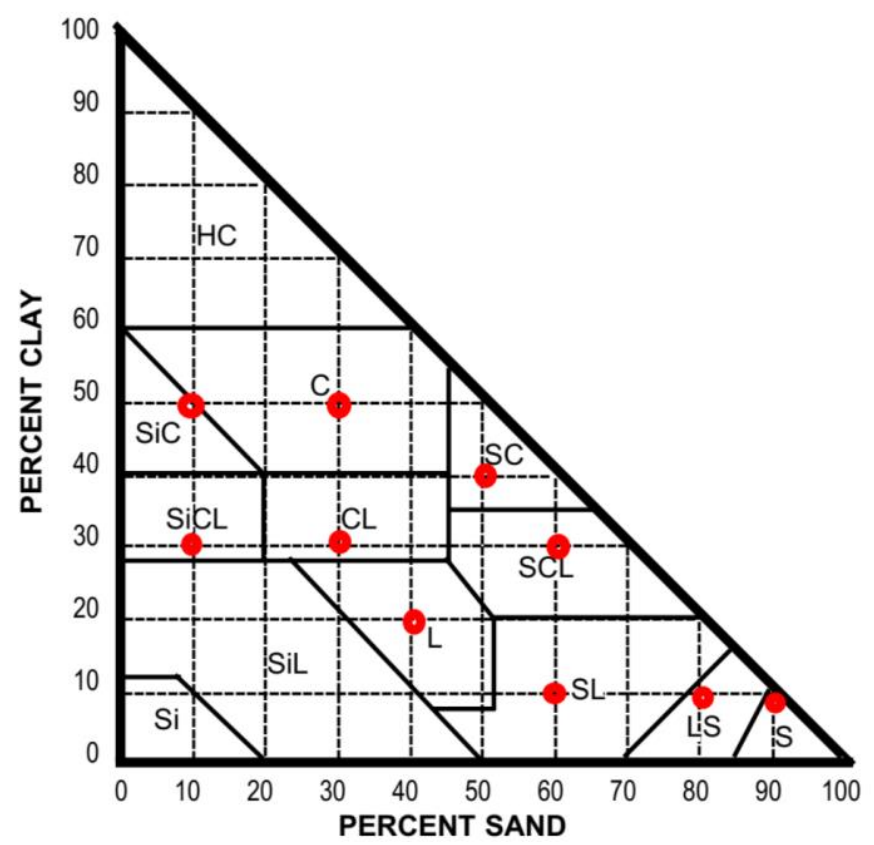

Fig. B1. Soil texture classes triangle (adapted from Soil Classification Working Group, 1998). Red circles show the allocated sand and clay percentages for each observed soil texture class (the remainder of each class is silt). Abbreviations for the texture classes are: $\mathrm{HC}$, heavy clay; C, clay; SiC, silty clay; $\mathrm{SiCL}$, silty clay loam; CL, clay loam; SC, sandy clay; SiL, silt loam; L, loam; SCL, sandy clay loam; SL, sandy loam; Si, silt; LS, loamy sand; S, sand.
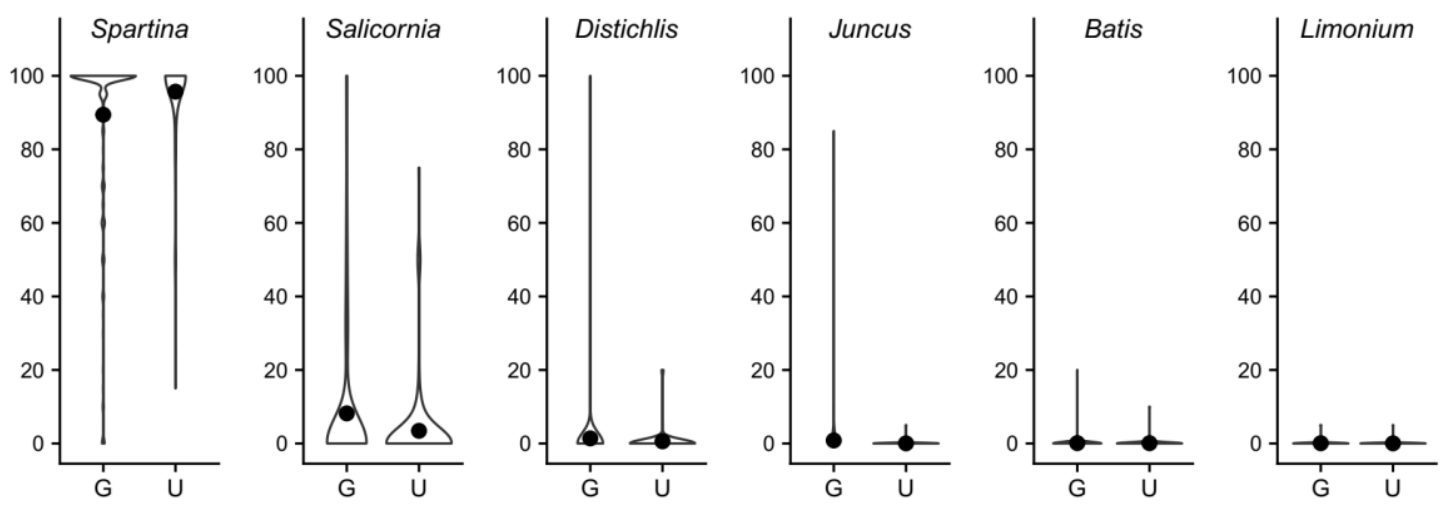

Fig. B2. Raw species data for observational survey marshes. Kernel density plots of percentage of live plant cover that is Spartina alterniflora, Salicornia spp., Distichlis spicata, Juncus roemerianus, Batis maritima and Limonium spp. in grazed $(G)$ and ungrazed $(U)$ quadrats. Black points = mean. 

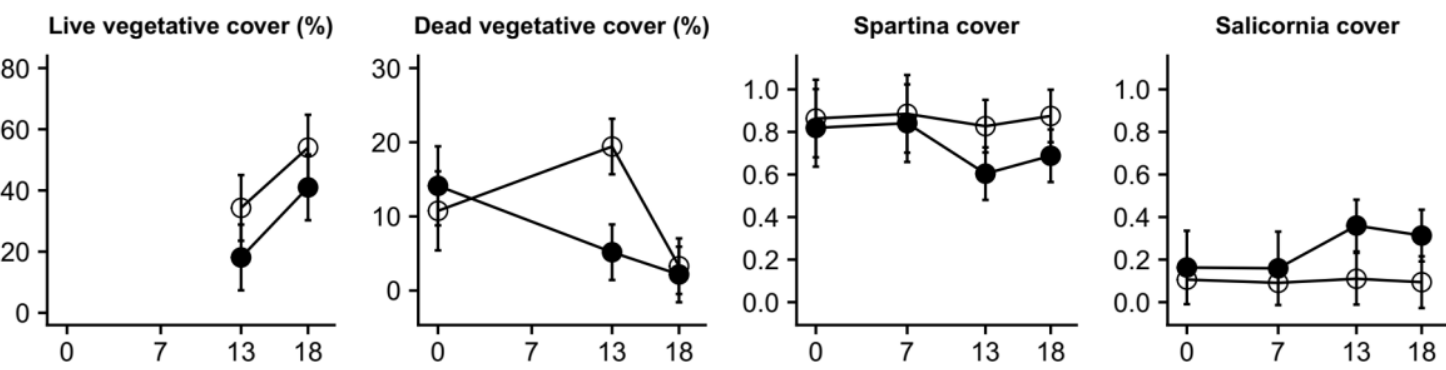

Stem height $(\mathbf{c m})$ (Log)Stems flowering

Benthic algae (blue-green) (Log)Adult fiddler crabs
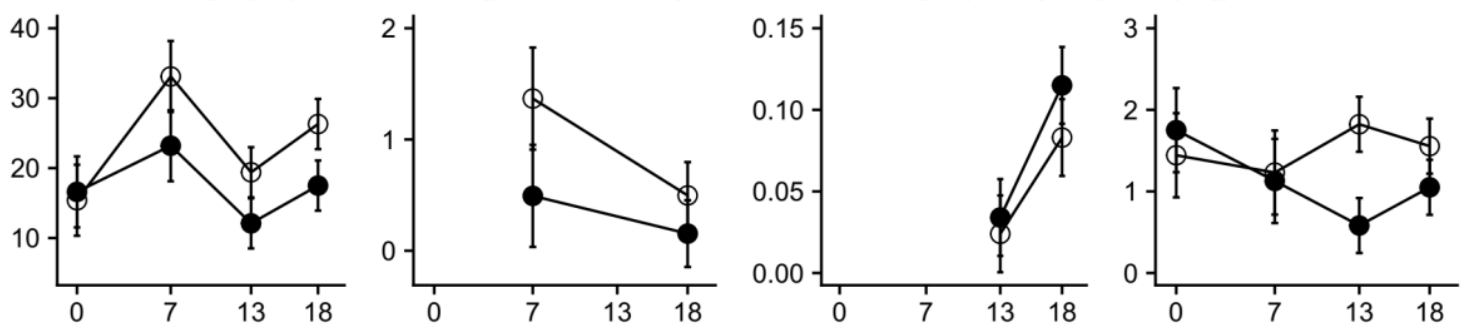

Fig. B3. Changes over time for all responses for which grazing treatment was significant at 18 months. Points show predicted means $\pm 95 \%$ confidence intervals. Spartina and Salicornia cover were recorded as proportion of total live plant cover. Benthic algae concentrations $=\mu \mathrm{g} \mathrm{chl-a} \mathrm{cm}{ }^{-2}$. The densities of flowering stems and fiddler crabs were recorded in a $0.3 \times 0.3 \mathrm{~m}$ quadrat (although predictions here are on the log scale). 


\section{Appendix C: Supporting information for Chapter 4}

\section{Contents}

Supplementary methods. Allocation of grazing levels

Supplementary methods. Calculating surrounding landscape 162

Supplementary methods. Terrestrial surveys for GMEP 162

Supplementary methods. Statistical packages 163

$\begin{array}{ll}\text { Table C1. Study site details } & 164\end{array}$

Table C2. Visual indicators used to allocate qualitative grazing levels 166

Table C3. Simplification of broad habitat types 166

$\begin{array}{ll}\text { Table C4. Bee abundance data for each marsh } & 167\end{array}$

Table C5. Insect-pollinated plant species on study saltmarshes 168

Table C6. Net effect of grazing on bee community 169

Table C7. Mechanisms of grazing effect on bee community, all insect- 170 pollinated plant species combined

Table C8. Mechanisms of grazing effect on bee community via key plant 172 species

$\begin{array}{ll}\text { Table C9. } \beta \text {-diversity analyses } & 174\end{array}$

Table C10. Comparison with terrestrial habitats 175

Fig. C1. Allocation of grazing levels based on dung counts and 176 qualitative assessment

Fig. C2. Network tested using LMMs with key plant species separated 176

Fig. C3. Bee species by abundance and prevalence 177

Fig. C4. Flower cover and bee visits, as a proportion of total 177

Fig. C5. Model predictions for plant responses to grazing 178

Fig. C6. Model predictions for bee responses to grazing and vegetation 179

Fig. C7. $\beta$-diversity analyses 180 


\section{Supplementary methods: Allocation of grazing levels quantitatively and}

\section{qualitatively}

Grazing levels were allocated both quantitatively and qualitatively to every transect on every visit, and combined to obtain an overall relative grazing level for each marsh. The grazing level was assessed quantitatively at each transect by counting the number of dung piles per grazer species lying $1 \mathrm{~m}$ to either side of the transect line. For each species (cattle, pony, sheep), we rescaled the dung counts on a scale of 0-1, based on the maximum observed value across all surveys, and summed them to get a total for the transect (potential range 0-3).

Rescaled dung count $=\frac{\text { no. cattle droppings }}{\text { max no. cattle droppings }}+\frac{\text { no. pony droppings }}{\text { max no. pony droppings }}+\frac{\text { no. sheep droppings }}{\text { max no. sheep droppings }}$ We averaged these dung counts across every transect and visit to get a single dung count value for the surveyed areas of the marsh. Due to tidal action, dung may be washed off or deposited on the marsh, depending on the stage in the spring-neap tidal cycle. Therefore, we also made a qualitative assessment of the grazing level at each transect on every visit (0: ungrazed, 1: lightly, 2: moderately, 3: heavily) based on visual indicators such as animal presence, droppings, damage to ground and vegetation (Table $\mathrm{C} 2$ ). We averaged the qualitative grazing levels across every transect and visit to obtain a single qualitative grazing level for the surveyed areas of the marsh (potential range 0-3).

There was a strong positive correlation between the grazing level assessed dung counts and the grazing level assessed qualitatively (Kendall's tau correlation test, tau $=0.88, p<.001$ ). We allocated each marsh to one of three grazing categories (absent, extensive, intensive grazing) based on these scores (Fig. C1). The allocated grazing levels are relative and not defined in terms of livestock units (LUs). Where LUs could be reasonably estimated for the entire marsh (e.g. on enclosed marsh areas) we have noted these in Table $\mathrm{C} 1$. Intensively grazed marshes were characterised by having more damage to the ground, more visible droppings, and more areas of vegetation that had been closely and uniformly cropped by grazers: in the intensively grazed marshes at least a quarter of all quadrats (ranging $29-74 \%$ in the four marshes) had a sward height of $<8 \mathrm{~cm}$, whereas fewer than a quarter of quadrats (15-24\% per marsh) in extensively grazed marshes had such a short sward. In the four ungrazed marshes, the percentage of quadrats with this short sward ranged from $0-11 \%$. 
Supplementary methods: Calculating surrounding landscape - proportion of natural habitat

Surrounding landscape can affect bee visitation rates and diversity, both responses declining with increasing distance from natural habitat (Ricketts et al. 2008; Viana et al. 2012). Species sensitivity to land use is dependent on species traits such as foraging range and sociality (De Palma et al. 2015) and different guilds are affected by landscape context at different spatial scales (Steffan-Dewenter et al. 2002). To allow us to account for landscape effects in our models of bee abundance and bee diversity, we calculated the proportion of natural habitat surrounding each transect at a range of scales. We used a 2015 land cover map (Rowland et al. 2017) which provides classification of UK land cover based on UK BAP broad habitat types at $25 \mathrm{~m}$ resolution. We classified each broad habitat type as either semi-natural vegetated habitat (hereafter 'natural habitat') or not, as defined in Table C3. We then calculated the proportion of surrounding landscape that is natural habitat for each marsh at a range of radii from $250 \mathrm{~m}$ to $3 \mathrm{~km}(250,500,750,1000,1500,2000$, $3000 \mathrm{~m})$. Each radius was tested individually in our LMMs, and assessed on the basis of $\triangle \mathrm{AIC}$ compared with no landscape term. Inclusion of the landscape variable did not improve any of our models. This may be due to the dominance of honeybees and bumblebees in our data: multiple studies have shown that these guilds are less affected by landscape context than non-Bombus wild bees (Steffan-Dewenter et al. 2002; Garibaldi et al. 2011; Murray et al. 2012; Bennett et al. 2014).

All GIS analyses were conducted in ArcGIS 10.5.1.

\section{Supplementary methods: Terrestrial surveys for GMEP}

Pollinator surveys were conducted by the Centre for Ecology and Hydrology as part of the Glastir Monitoring \& Evaluation Programme (GMEP) for the Welsh Government (Emmett et al. 2017). GMEP included two pollinator surveys - one in July and one in August - in each of $3001 \mathrm{~km}$ squares in Wales. Each square was visited only once from 2013 to 2016, in the first round of a rolling environmental survey. Of the 300 squares, 150 comprised a stratified random sample to represent land classes across Wales, and 150 comprised a "targeted component" and were selected based on various criteria associated with the Glastir agri-environment scheme. Within each site, two $1 \mathrm{~km}$ transects, divided into roughly $200 \mathrm{~m}$ sections, were surveyed for bees between 10am and $4 \mathrm{pm}$ on warm, dry, calm days following the UK Wider Countryside Butterfly Survey (UKBMS 2019) method. Transects were walked at an even pace, and all bees that were observed within a $5 \mathrm{~m}$ box around the observer were recorded and identified as honeybee, bumblebee or 'solitary bee' (i.e. nonBombus wild bee). Temperature and wind speed (Beaufort scale) were recorded for each transect. For more information on square selection and survey methodology, see the GMEP reports and appendices (GMEP 2019). 
A habitats survey was carried out in the GMEP squares during the same years as the pollinator surveys. Every accessible land parcel in the square was assigned a broad habitat type according to UK Biodiversity Action Plant broad habitat types (JNCC 2019). We extracted the underlying broad habitat throughout every $200 \mathrm{~m}$ pollinator transect section by intersecting with habitat polygons in ArcGIS Desktop 10.6 (ESRI, Redlands, California). The broad habitat which accounted for the greatest proportion of each $200 \mathrm{~m}$ transect section was allocated as the dominant underlying habitat. Transect sections were only included in these analyses if $>100 \mathrm{~m}$ intersected the allocated habitat type. Furthermore, we only assessed habitats with $>50$ transect sections.

\section{Supplementary methods: Statistical packages}

LMMs were fitted using Ime4 (Bates et al. 2015). We used ImerTest (Kuznetsova et al. 2017) to calculate t-test P-values for comparison of different factor levels, and piecewiseSEM (Lefcheck 2016) to calculate $R^{2}$ values. Negative binomial GLMMs were fitted using glmmTMB (Brooks et al. 2017) and sjsstats (Lüdecke 2019) was used to calculate $R^{2}$ values. We used the DHARMa package (Hartig 2019) for residual checks and tests for goodness of fit and zero-inflation. (G)LMM graphs were plotted using visreg (Breheny and Burchett 2017) and ggplot2 (Wickham 2016) and 95\% confidence intervals were estimated by parametric bootstrapping using the bootpredictIme4 package (Duursma 2019) which implements the Ime4 bootMer function in visreg. For beta-diversity analyses, pairwise distance matrices were calculated from presence-absence species data using the betapart package in $R$ (Baselga and Orme 2012). PERMANOVA tests were conducted using the adonis command in the vegan package (Oksanen et al. 2019) in R. Post-hoc tests for PERMANOVA were performed using pairwise.perm.manova in RVAideMemoire (Hervé 2019) with fdr-adjusted P-values. PERMDISP tests were conducted using betadisper and permutest in vegan. All permutational tests were performed with 9999 permutations. 
Table C1.(overleaf). Study site details . Grazing management and environmental characteristics for the 11 study sites. Livestock Units per hectare $\left(\mathrm{LUha}^{-1}\right)$ estimated for the entire marsh (not only the surveyed areas) during the main summer grazing period, where possible, based on information from landowners or animal head counts. Cattle $=1$, ponies $=0.5$, sheep $=0.12$ LU (Welsh Government 2013b). National Vegetation Classification (NVC, a systemic catalogue of the plant communities of Great Britain, Rodwell 2006) based on 1998 NVC Survey data (GIS shapefile provided by Natural Resources Wales) and confirmed by field observations. Marsh geomorphology was classified according to Allen (2000) and estuary position according to Adam (1990). Duration of current grazing levels was obtained from land managers/owners, but several had no knowledge of grazing management prior to their tenancy, therefore figures shown are a minimum. Sediment types defined as L-CL (loam-clay) or S (sandy). Adjacent habitat is shown for surrounding $1 \mathrm{~km}$, calculated from a 2015 land cover map (Rowland et al. 2017) and classified according to Table C2: Ag=agricultural land, Bu=built-up areas, $\mathrm{Na}=$ natural or semi-natural areas, $\mathrm{Op}=$ open water or littoral zone.

*LW not surveyed in mid-summer 2016. Both LW \& PM had extremely large low marsh zones, and it was not safe or practical to distribute transects evenly across these marshes due to dense creek networks in the lower marsh areas. However, we did sample within the low marsh vegetation community at both these sites.

$\S$ Intensively-grazed marshes were, on average, larger than marshes of the other grazing categories, possibly because larger marsh areas are more convenient for graziers. This could influence bee use (e.g. due to longer flight distances to the lower regions of these marshes). However, the smallest intensive marsh (TR) was also the least-foraged (Table C4), and the majority of bee visits in the most foraged intensive marsh (PM, Table C4) were recorded on the most seaward transect, therefore we do not believe this was a factor.

tSediment-type from Skov et al. (2016)

‡ Sediment-type from field survey following methods in Skov et al. (2016) 


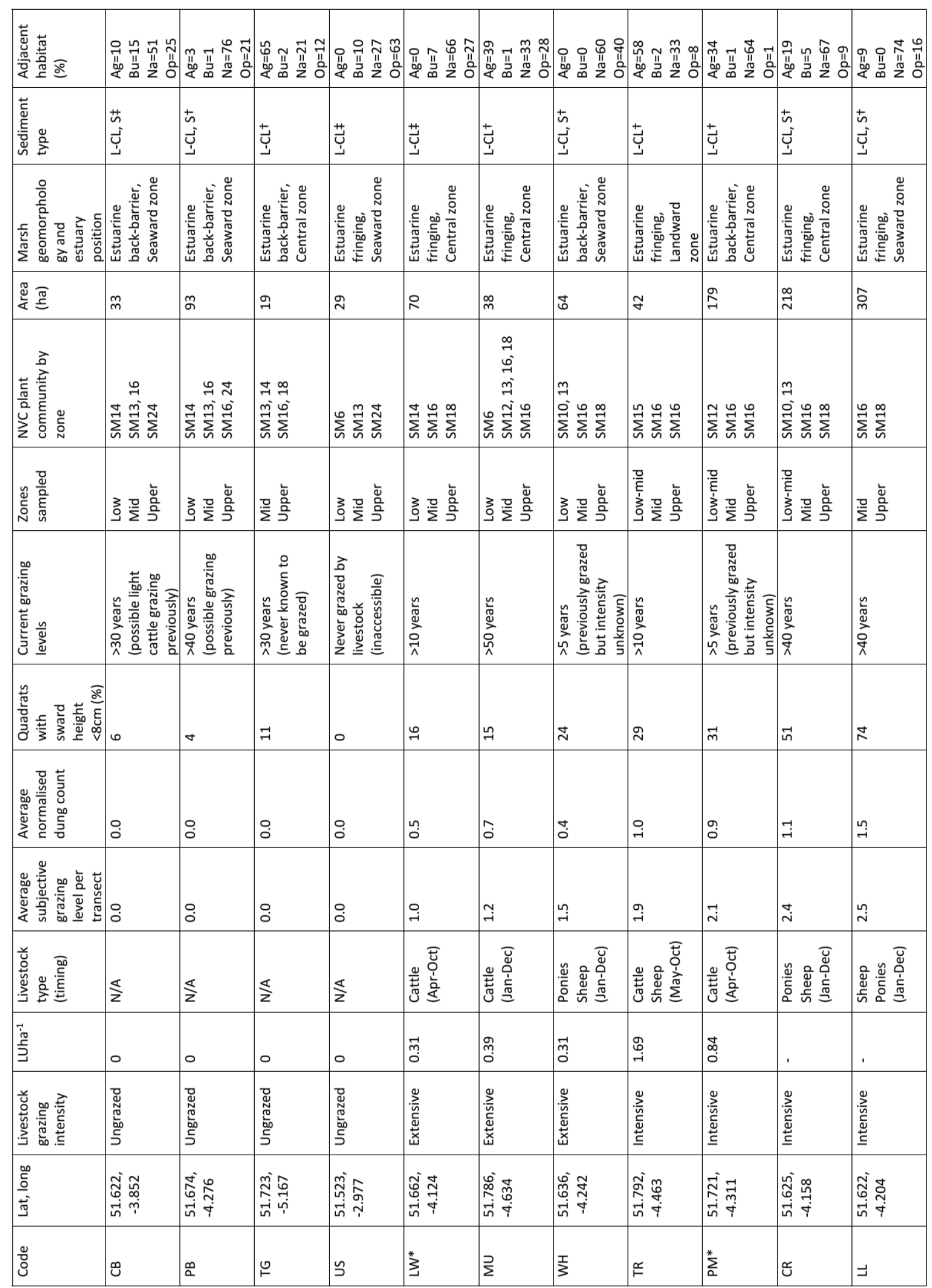


Table C2. Visual indicators used to allocate qualitative grazing levels. Each descriptor is not absolute, but was used in combination to allocate the best representative grazing level to the area immediately surrounding each transect (within a few hundred metres, approx. 5 ha).

\begin{tabular}{|lllll|}
\hline Indicator & 0: Ungrazed & 1: Lightly grazed & 2: Moderately grazed & 3: Heavily grazed \\
\hline $\begin{array}{l}\text { Animal } \\
\text { presence }\end{array}$ & None & $\begin{array}{l}\text { Area accessible to } \\
\text { animals but no } \\
\text { more than a few } \\
\text { animals present }\end{array}$ & $\begin{array}{l}\text { Small herd (e.g. 5-10 } \\
\text { cattle/ponies, 15-30 } \\
\text { sheep) present }\end{array}$ & $\begin{array}{l}\text { Many animals (e.g. }>20 \\
\text { cattle/ponies, }>50 \\
\text { sheep) present }\end{array}$ \\
\hline Droppings & None & Infrequently seen & Commonly seen & Abundant across area \\
\hline $\begin{array}{l}\text { Damage to } \\
\text { ground }\end{array}$ & None & $\begin{array}{l}\text { Hoofprints rarely } \\
\text { seen. Very little } \\
\text { bare ground. }\end{array}$ & $\begin{array}{l}\text { Hoofprints/poaching } \\
\text { commonly seen. } \\
\text { Some bare ground. }\end{array}$ & $\begin{array}{l}\text { Ground is badly } \\
\text { poached or extremely } \\
\text { compacted. Areas of } \\
\text { bare ground common. }\end{array}$ \\
\hline $\begin{array}{l}\text { Sward height } \\
\text { and damage to } \\
\text { vegetation }\end{array}$ & $\begin{array}{l}\text { Majority of sward } \\
\text { longer than 15cm, } \\
\text { complex structure, } \\
\text { no visible cropping }\end{array}$ & $\begin{array}{l}\text { Majority of sward } \\
\text { longer than } \\
\text { 15cm, complex } \\
\text { structure, little } \\
\text { visible cropping }\end{array}$ & $\begin{array}{l}\text { Some areas shortly } \\
\text { cropped (< 10 cm) } \\
\text { interspersed with } \\
\text { longer, more tussocky } \\
\text { areas }\end{array}$ & $\begin{array}{l}\text { Majority of vegetation } \\
\text { cropped to a short, } \\
\text { uniform sward; only } \\
\text { unpalatable species are } \\
\text { above } \text { 10cm }\end{array}$ \\
\hline
\end{tabular}

Table C3. Simplification of broad habitat types.

\begin{tabular}{|c|c|c|}
\hline Broad habitat type & Classification & Natural habitat \\
\hline Broadleaved woodland & Natural or semi-natural vegetated habitat & $\checkmark$ \\
\hline Coniferous woodland & Natural or semi-natural vegetated habitat & $\checkmark$ \\
\hline Arable and horticulture & Agricultural land & - \\
\hline Improved grassland & Agricultural land & - \\
\hline Neutral grassland & Natural or semi-natural vegetated habitat & $\checkmark$ \\
\hline Calcareous grassland & Natural or semi-natural vegetated habitat & $\checkmark$ \\
\hline Acid grassland & Natural or semi-natural vegetated habitat & $\checkmark$ \\
\hline Fen, Marsh and Swamp & Natural or semi-natural vegetated habitat & $\checkmark$ \\
\hline Heather & Natural or semi-natural vegetated habitat & $\checkmark$ \\
\hline Heather grassland & Natural or semi-natural vegetated habitat & $\checkmark$ \\
\hline Bog & Natural or semi-natural vegetated habitat & $\checkmark$ \\
\hline Inland rock & Natural or semi-natural vegetated habitat & $\checkmark$ \\
\hline Saltwater & Open water or littoral zone & - \\
\hline Freshwater & Open water or littoral zone & - \\
\hline Supra-littoral rock & Natural or semi-natural vegetated habitat & - \\
\hline Supra-littoral sediment & Natural or semi-natural vegetated habitat & - \\
\hline Littoral rock & Open water or littoral zone & - \\
\hline Littoral sediment & Open water or littoral zone & - \\
\hline Saltmarsh & Natural or semi-natural vegetated habitat & $\checkmark$ \\
\hline Urban & Built-up areas & - \\
\hline Suburban & Built-up areas & - \\
\hline Wetland* & Natural or semi-natural vegetated habitat & $\checkmark$ \\
\hline
\end{tabular}

* Land managed by RSPB Newport Wetlands was classified as Arable and Horticultural in the Land Cover 2015 map which is incorrect. We renamed these areas as Wetland in our GIS maps, classified under Natural or semi-natural vegetated habitat. 
Table C4. Bee abundance data for each marsh. Average number of foraging bees observed per km on each marsh, by species. The Andrena specimen could not be identified to species level due to the quality of the specimen.

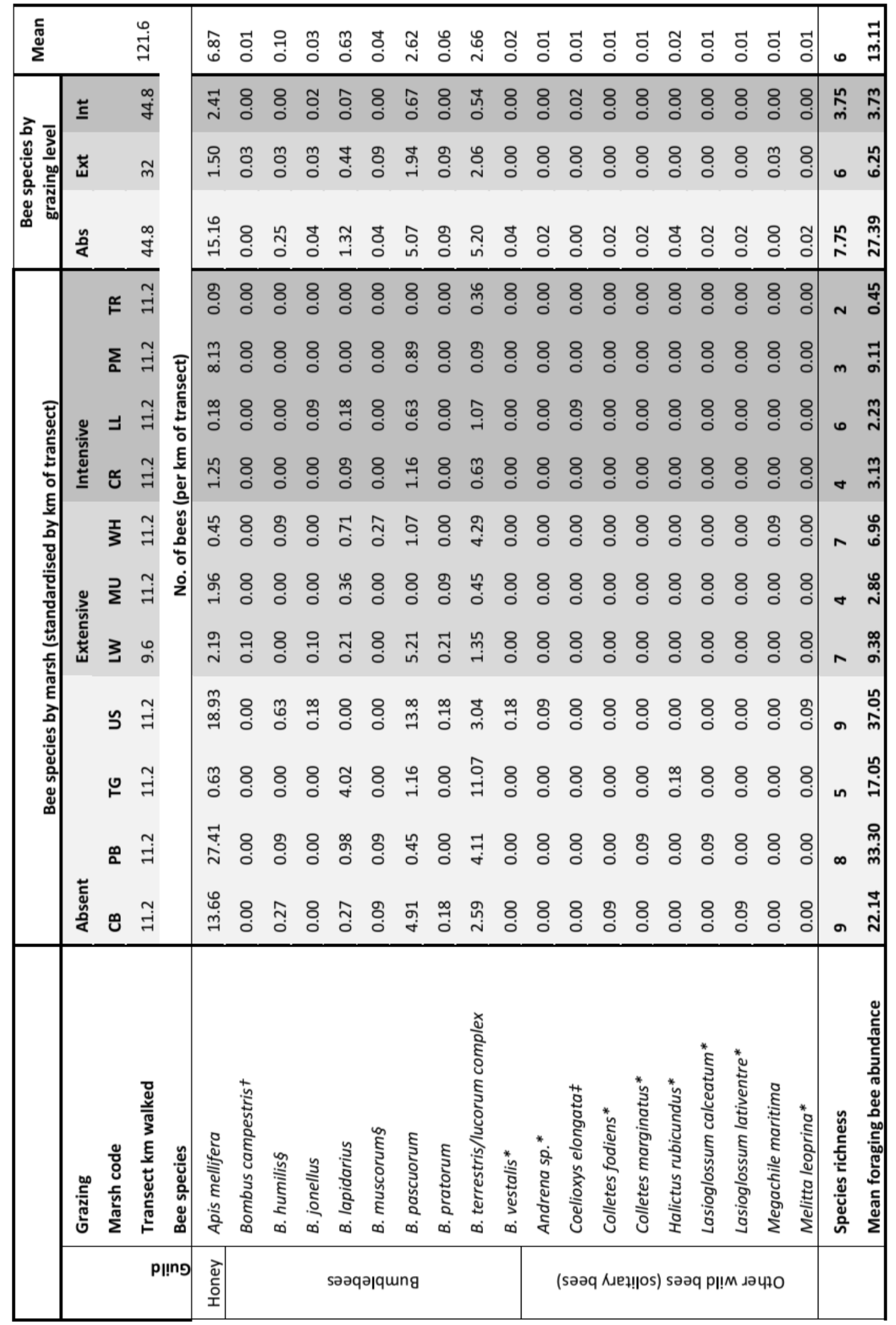

* Species unique to ungrazed marshes; † Species unique to extensively-grazed marshes; $¥$ Species unique to intensively-grazed marshes; § Section 7 Species, Environment (Wales) Act 2016 
Table C5. Insect-pollinated plant species on study saltmarshes. Flower cover per plant species, and bee visits per plant species for each marsh.

\begin{tabular}{|c|c|c|}
\hline 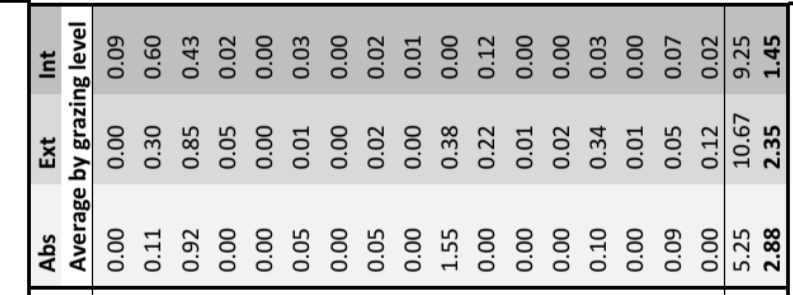 & 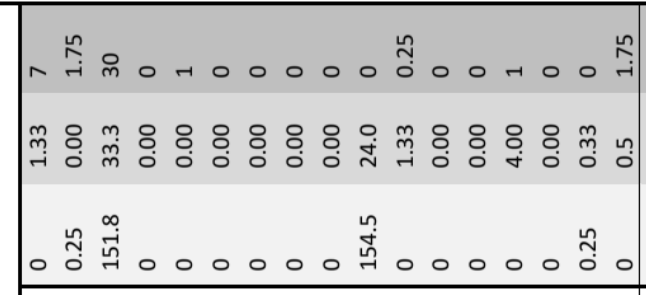 & 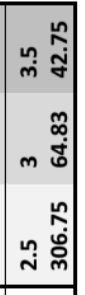 \\
\hline 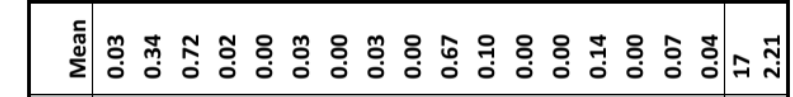 & 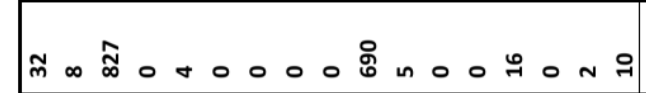 & o \\
\hline 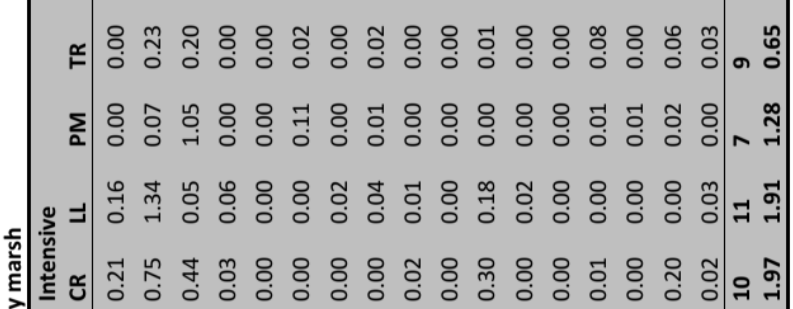 & 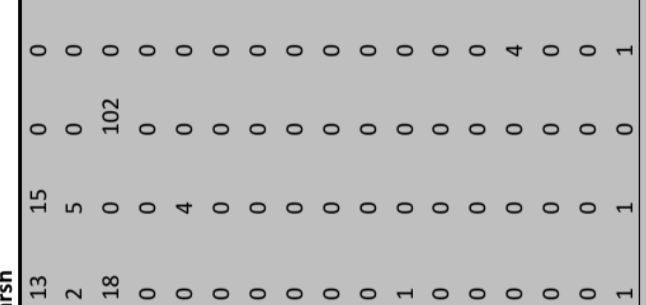 & 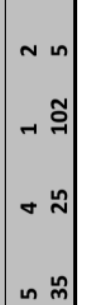 \\
\hline 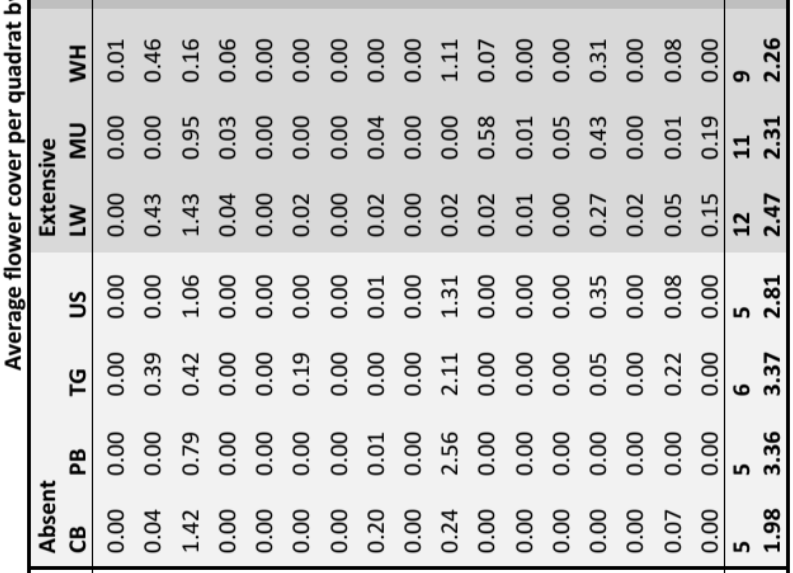 & 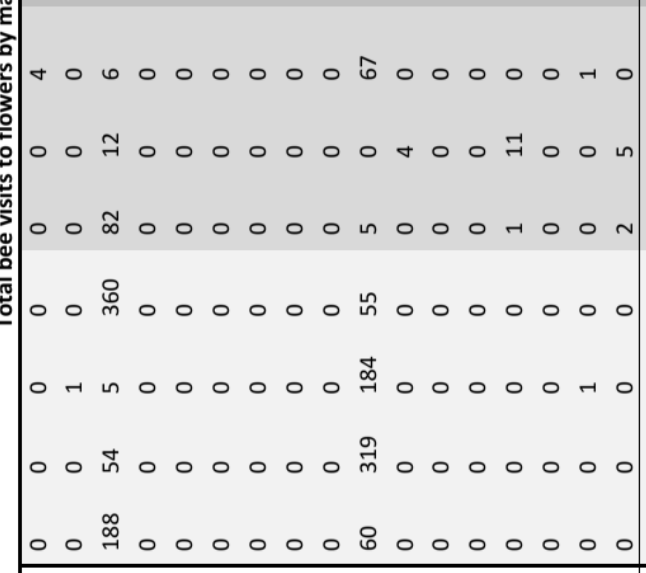 & 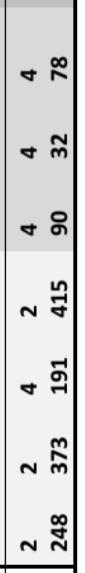 \\
\hline 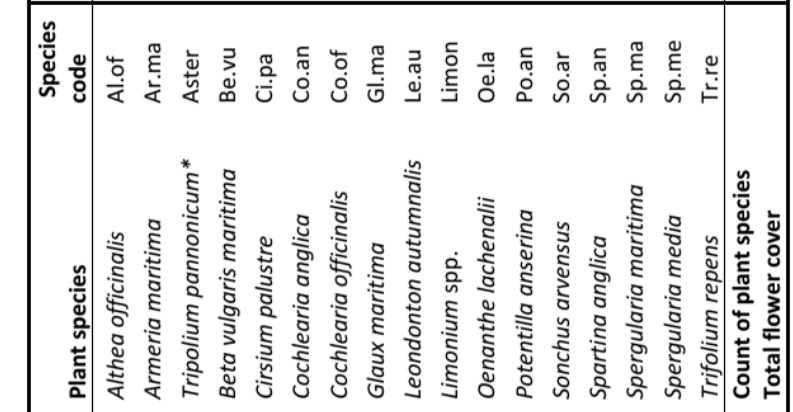 & 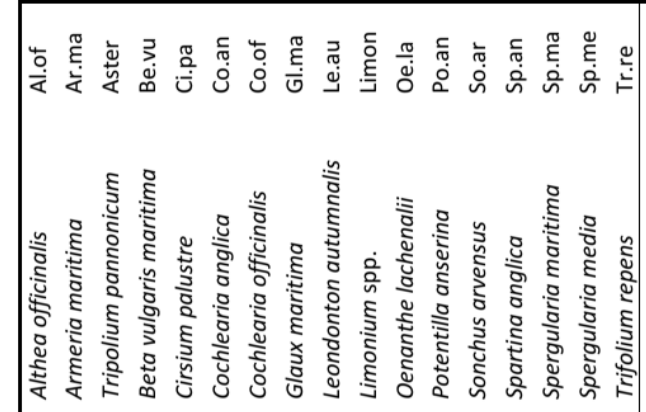 & 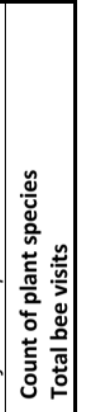 \\
\hline
\end{tabular}

*Formerly Aster tripolium 
Table C6. Net effect of grazing on bee community. Results of LMMs to test net effect of grazing on bee abundance, bee species richness, and bee Shannon $\mathrm{H}$ diversity. Initial models were simplified where possible by removing weather (wind speed, temperature, temperature ${ }^{2}$ ) and landscape (natural habitat) terms if this did not increase AIC. Significant predictors in the final model are highlighted in bold. Coefficients of categorical predictors relative to baseline values are arranged as follows. Grazing: ext vs abs, int vs abs; Year: 2017 vs 2016, 2018 vs 2016; Season: mid vs early, late vs early. Pairwise comparisons of factor levels were conducted only if that factor was significant in the likelihood ratio (LR) test. Marginal (Marg) $R^{2}$ values relate to fixed effects only, Conditional (Cond) $R^{2}$ values relate to fixed + random effects.

\begin{tabular}{|c|c|c|c|}
\hline$=$ & 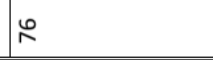 & 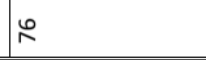 & 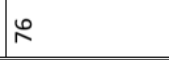 \\
\hline 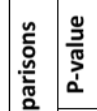 & 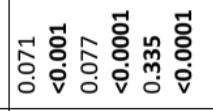 & 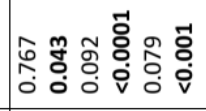 & 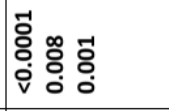 \\
\hline 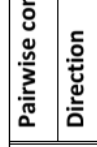 & 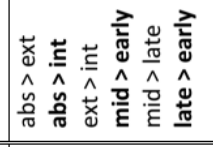 & 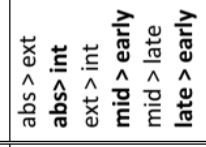 & 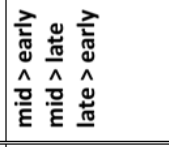 \\
\hline 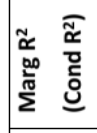 & 焉 & 商 & 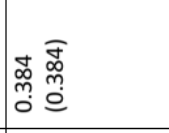 \\
\hline 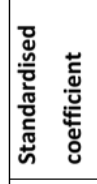 & 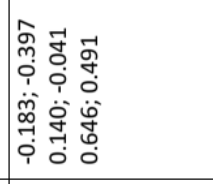 & 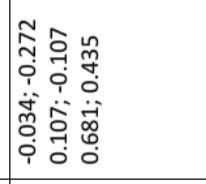 & 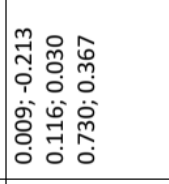 \\
\hline 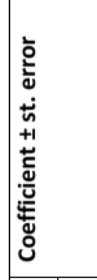 & 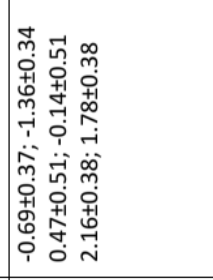 & 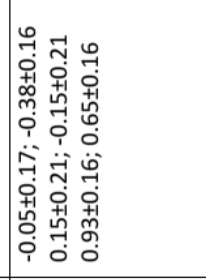 & 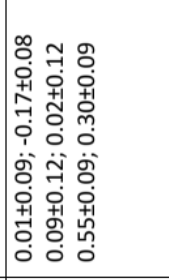 \\
\hline 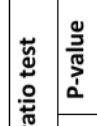 & 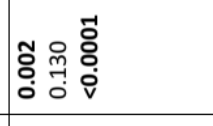 & 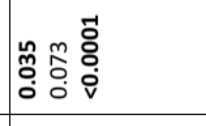 & 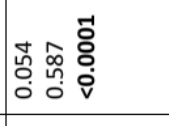 \\
\hline 苋 & N N & N N N & N N N \\
\hline$\underset{x}{\overline{\bar{u}}}$ & 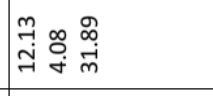 & 我 & مَ \\
\hline 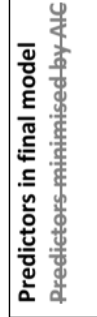 & 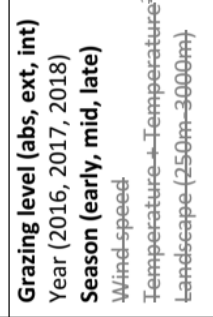 & 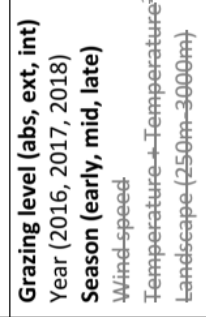 & 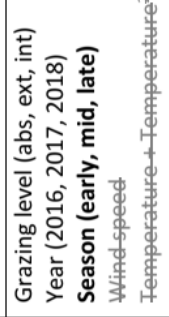 \\
\hline & 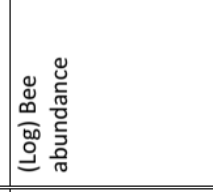 & 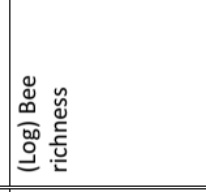 & 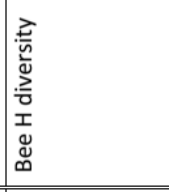 \\
\hline \begin{tabular}{|l}
$\overline{\mathrm{\Xi}}$ \\
¿ू \\
\end{tabular} & $\ll$ & $\infty$ & u \\
\hline
\end{tabular}


Table C7. Mechanism of grazing effects on bee community, all insect-pollinated plant (IPP) combined. Results of LMMs to test mechanisms of grazing effect on bee community. Initial models were simplified where possible by removing weather (wind speed, temperature, temperature ${ }^{2}$ ) and landscape (natural habitat) terms if this did not increase AIC. Significant predictors in the final model are highlighted in bold. Coefficients of categorical predictors relative to baseline values are arranged as follows. Grazing: ext vs abs, int vs abs; Year: 2017 vs 2016, 2018 vs 2016, 2018 vs 2017; Season: mid vs early, late vs early. Pairwise comparisons of factor levels were conducted only if that factor was significant in the likelihood ratio (LR) test. Marginal (Marg) $R^{2}$ values relate to fixed effects only, Conditional (Cond) $R^{2}$ values relate to fixed + random effects.

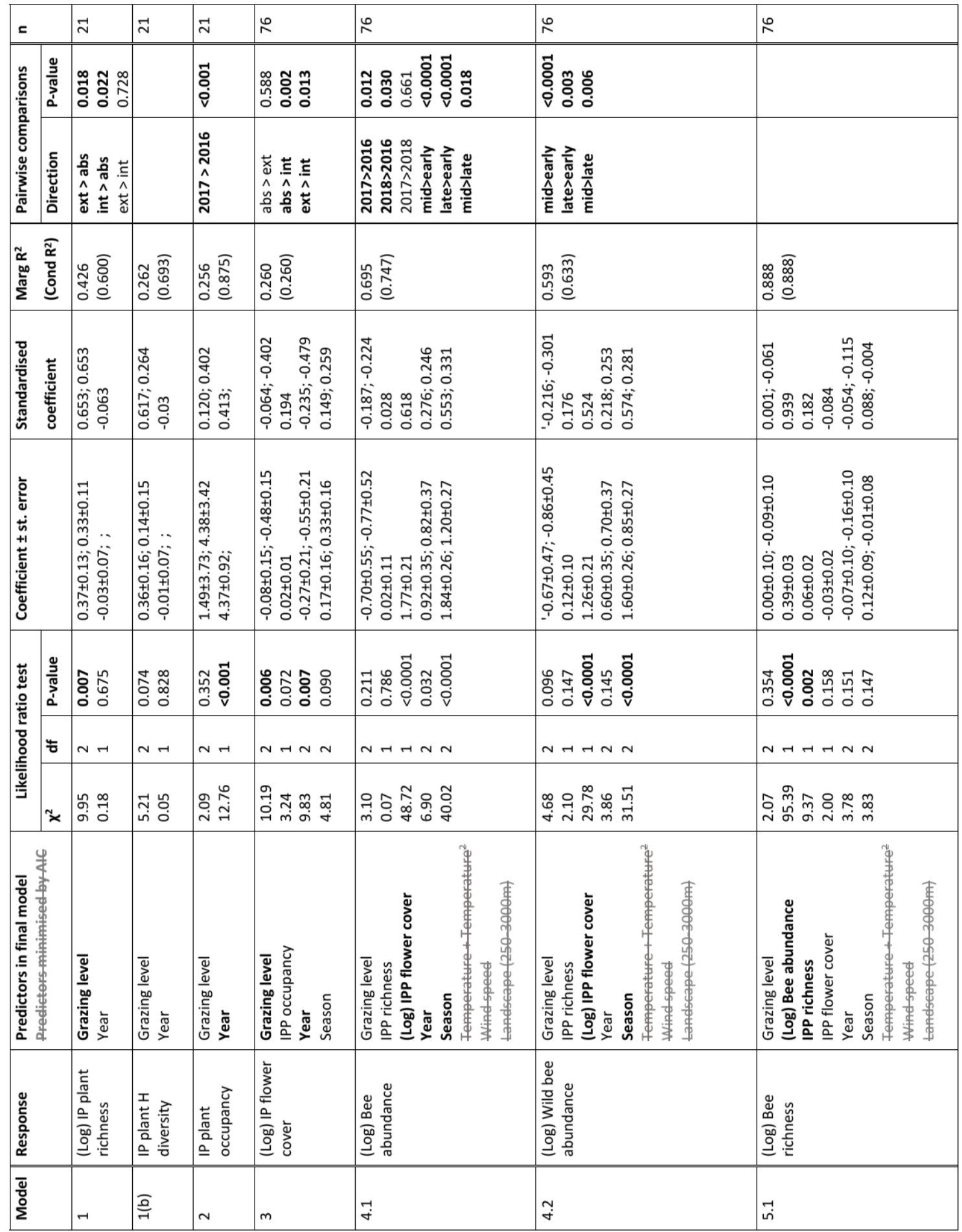


Table C7 cont.

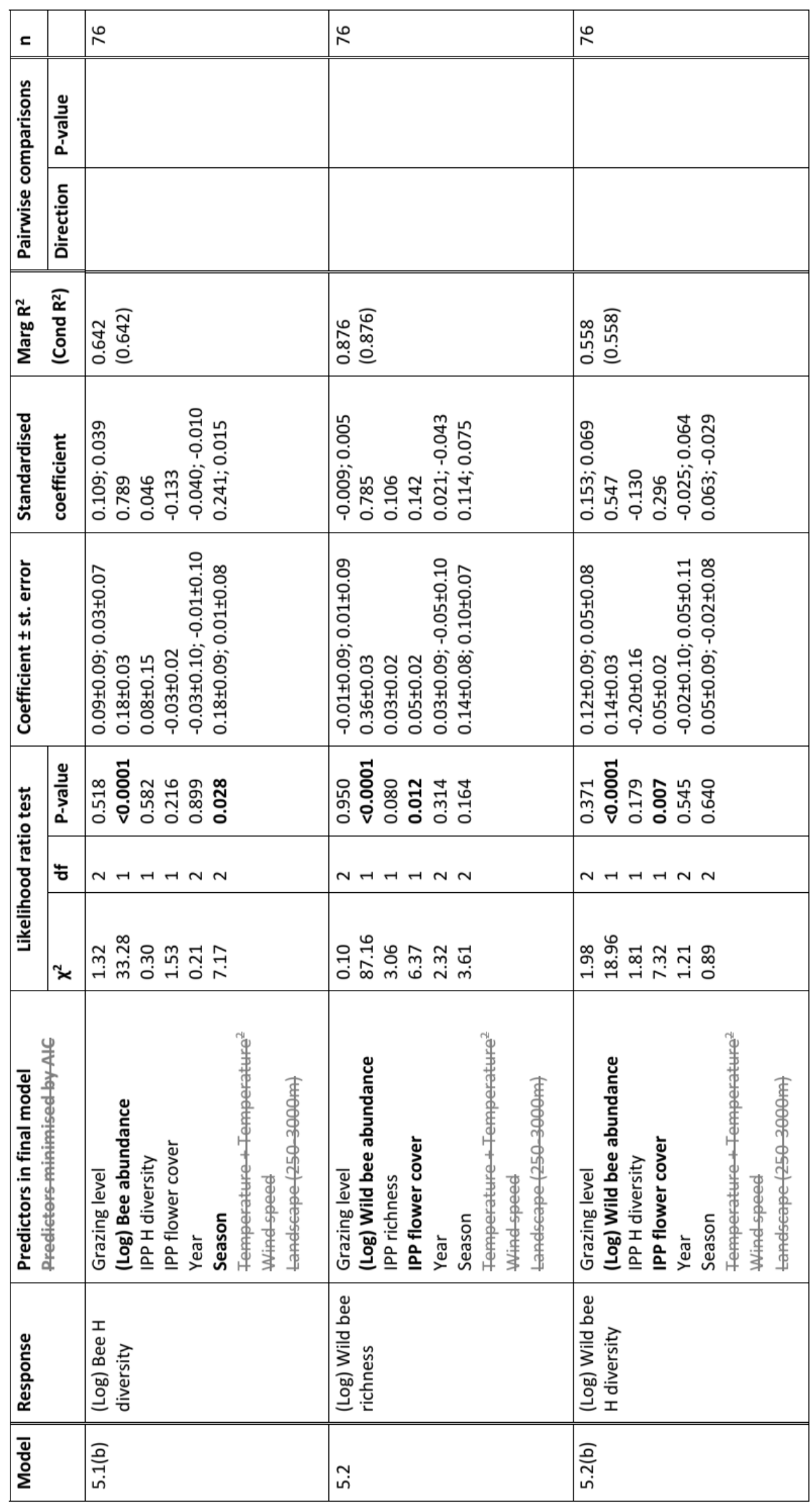


Table C8. Mechanism of grazing effects on bee community via key plant species. Results of LMMs to test mechanisms of grazing effect on bee community with Aster and Limonium separated from the other insect-pollinated plant (IPP) species. Initial models were simplified where possible by removing weather (wind speed, temperature, temperature ${ }^{2}$ ) and landscape (natural habitat) terms if this did not increase AIC. Significant predictors in the final model are highlighted in bold. Coefficients of categorical predictors relative to baseline values are arranged as follows. Grazing: ext vs abs, int vs abs; Year: 2017 vs 2016, 2018 vs 2016, 2018 vs 2017; Season: mid vs early, late vs early. Pairwise comparisons of factor levels were conducted by t-test, only if that factor was significant in the likelihood ratio test. Marginal (Marg) $R^{2}$ values relate to fixed effects only, Conditional (Cond) $R^{2}$ values relate to fixed + random effects.

\begin{tabular}{|c|c|c|c|c|c|c|c|c|c|c|}
\hline$=$ & A & $\overrightarrow{4}$ & A & $\overline{4}$ & 4 & ก & m & 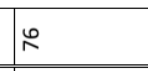 & 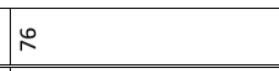 & 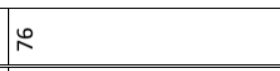 \\
\hline 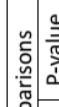 & 类 & & : & 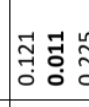 & & 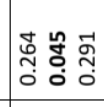 & 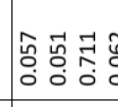 & 雚 & & \\
\hline 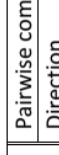 & 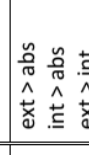 & & 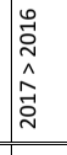 & 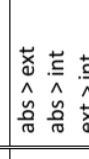 & 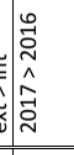 & 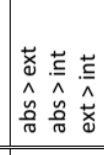 & 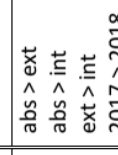 & 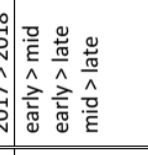 & & \\
\hline 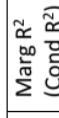 & 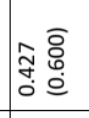 & 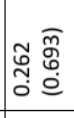 & 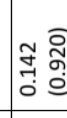 & 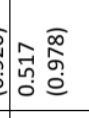 & 秴 & 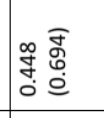 & 露商 & 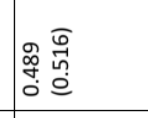 & $\begin{array}{ll}0 & \overline{2} \\
0 & 0 \\
0 & 0 \\
0\end{array}$ & 쿠응 \\
\hline 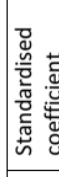 & 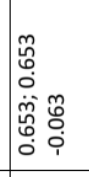 & 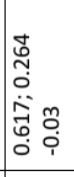 & 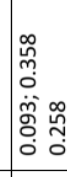 & 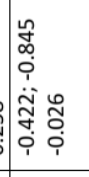 & 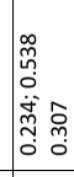 & 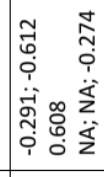 & 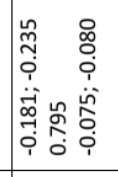 & 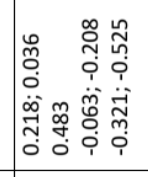 & 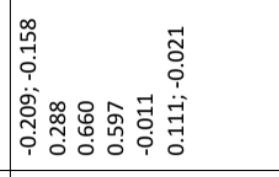 & 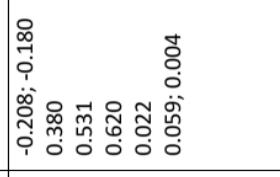 \\
\hline 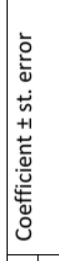 & 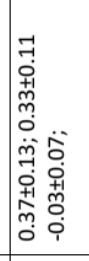 & 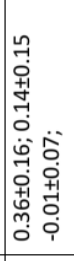 & 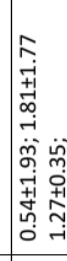 & 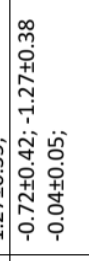 & 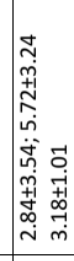 & 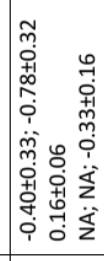 & 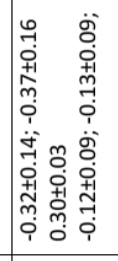 & 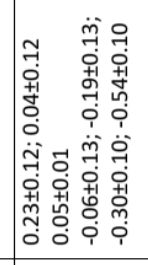 & 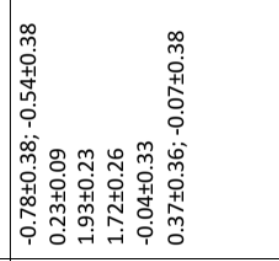 & 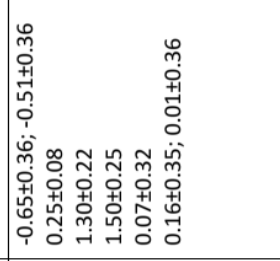 \\
\hline 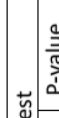 & bُ & $\begin{array}{lll}\pi & \infty \\
0 & 0 \\
0 & 0 \\
0 & 0 \\
\end{array}$ & 驾 : & 总导 & 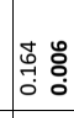 & 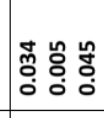 & 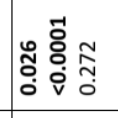 & 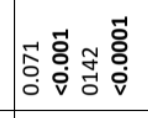 & 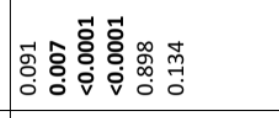 & 足 \\
\hline 尊 & ar & $4-4$ & $n-$ & 44 & $n-1$ & nat & $h+n$ & han- & NAनHAN & NAHAH \\
\hline $\mid$ & مू & సี & F & 冓 & $\overrightarrow{0}$ & 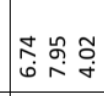 & 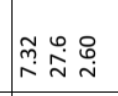 & 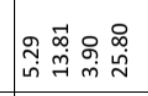 & 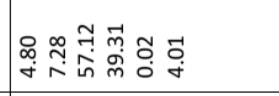 & 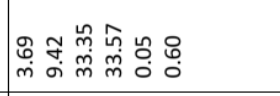 \\
\hline 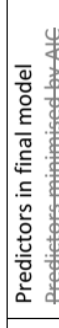 & 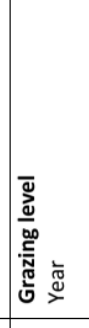 & 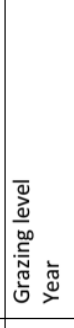 & 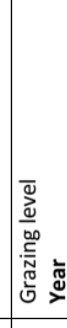 & 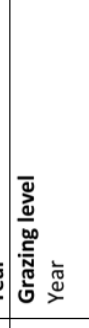 & 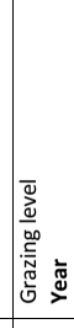 & 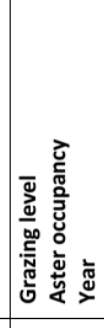 & 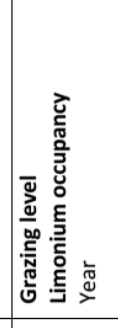 & 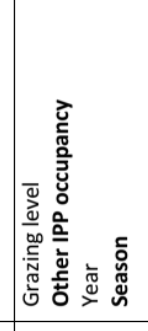 & 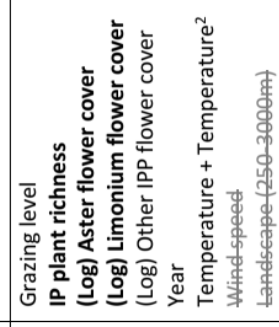 & 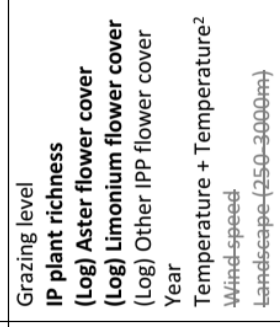 \\
\hline 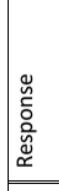 & 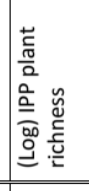 & 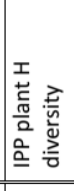 & 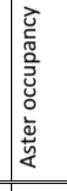 & 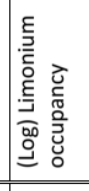 & 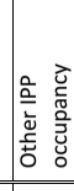 & 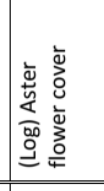 & 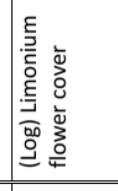 & 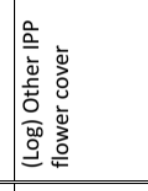 & 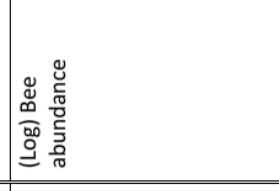 & 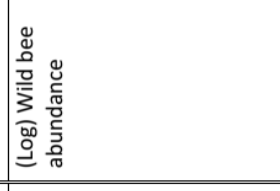 \\
\hline 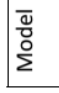 & - & 高 & $\bar{i}$ & $:=\bar{i}$ & $\stackrel{\Xi}{i}$ & $\bar{m}$ & 容 & 鋳 & $\bar{z}$ & 淁 \\
\hline
\end{tabular}


Table C8 cont.

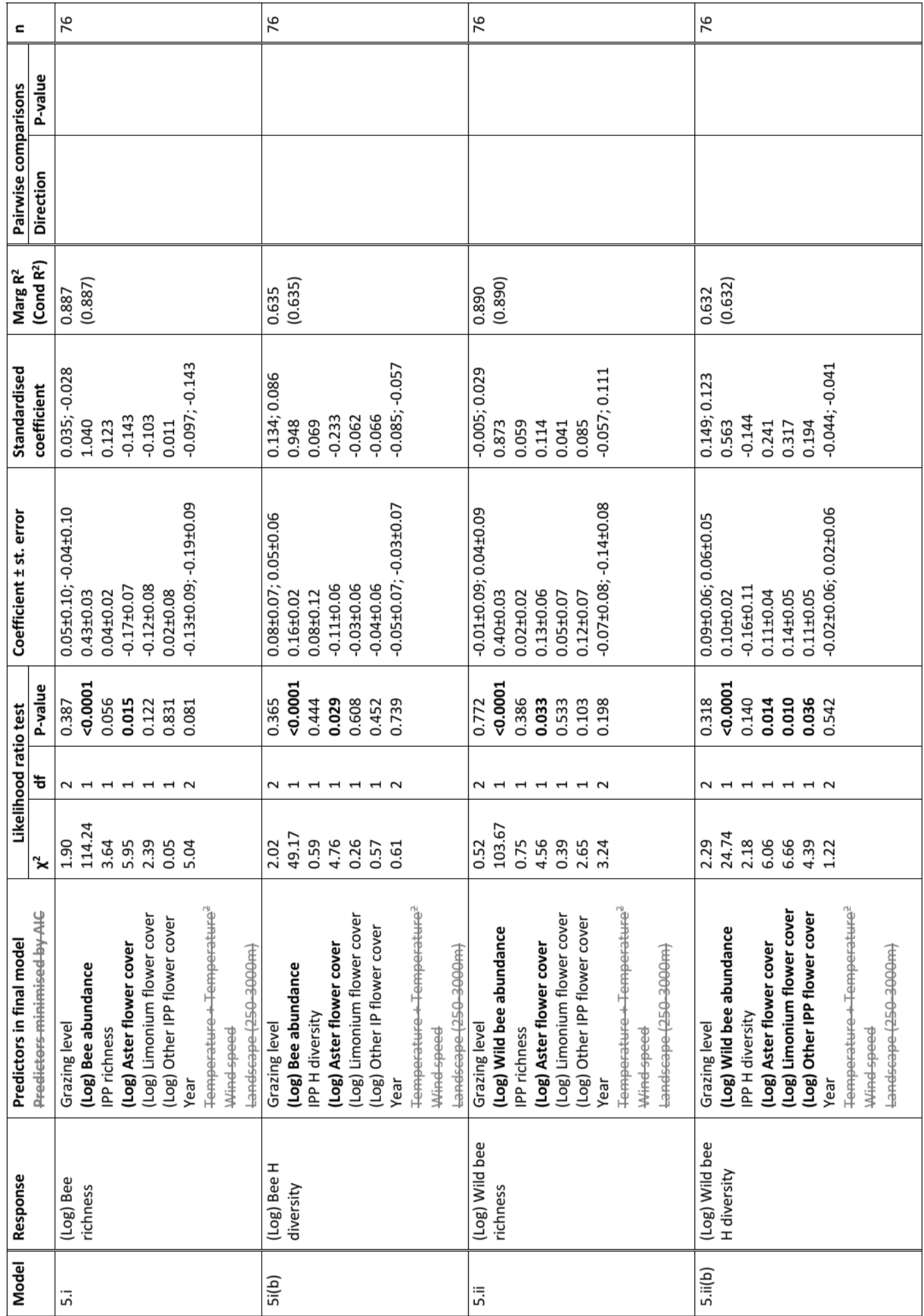


Table C9. $\beta$-diversity analyses. Results of PERMANOVA and PERMDISP analysis of partitioned $\beta$ diversity, for all bees and wild bees only.

\begin{tabular}{|c|c|c|c|c|c|c|c|}
\hline \multirow{5}{*}{ 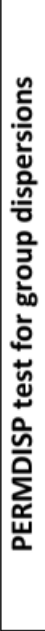 } & $a$ & & 㐘 & & & 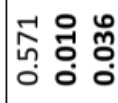 & \\
\hline & 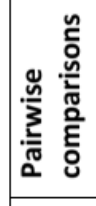 & & 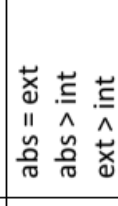 & & & 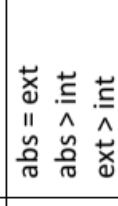 & \\
\hline & a & 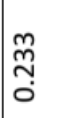 & ठ் & $\underset{\sim}{\tilde{N}}$ & 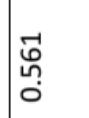 & ֻे & $\stackrel{n}{7}$ \\
\hline & 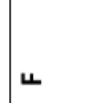 & ì & ¿̊ & ְְ & 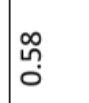 & \begin{tabular}{l}
\multirow{J}{0}{} \\
$\infty$
\end{tabular} & 오․ \\
\hline & t & N & N & N & N & $\sim$ & N \\
\hline \multirow{6}{*}{ 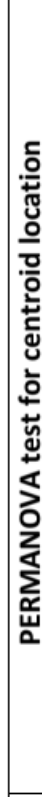 } & 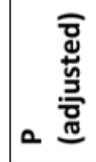 & & & 落 & & & 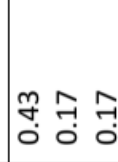 \\
\hline & 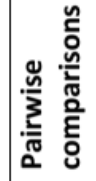 & & & 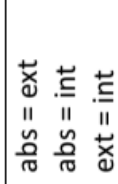 & & & 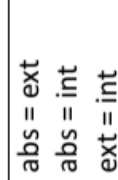 \\
\hline & a & 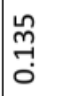 & 㐫 & Ỹ & 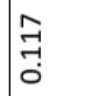 & 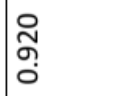 & 帒 \\
\hline & $\approx$ & న్ & 궁 & חึ & స్ & 峞 & f \\
\hline & ᄂ & 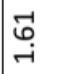 & m̧ & 帒 & ت & ํㅗํ & $\underset{m}{\stackrel{N}{m}}$ \\
\hline & t) & N & N & $N$ & $N$ & $\sim$ & $\sim$ \\
\hline \multicolumn{2}{|c|}{\begin{tabular}{|l}
$\frac{0}{0}$ \\
$\frac{0}{60}$ \\
$\frac{\pi}{10}$ \\
$\frac{10}{3}$
\end{tabular}} & 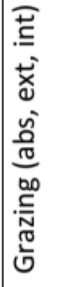 & 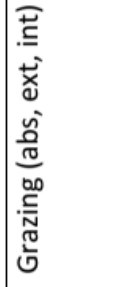 & 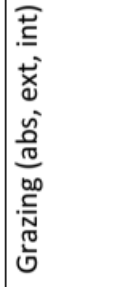 & 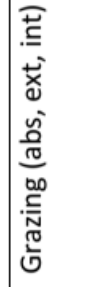 & 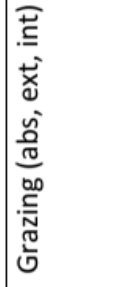 & 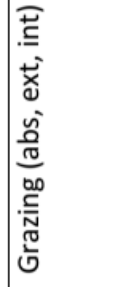 \\
\hline 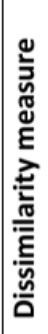 & & 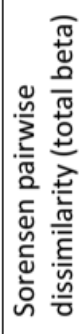 & 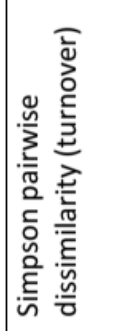 & 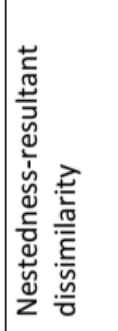 & 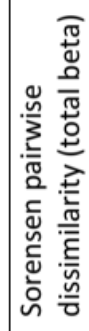 & 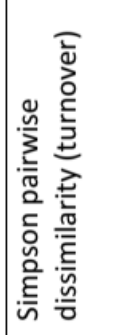 & 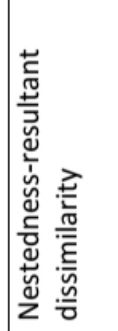 \\
\hline 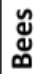 & & $\bar{\varepsilon}$ & $\bar{\varangle}$ & $\bar{\varangle}$ & $\frac{0}{3}$ & $\frac{0}{3}$ & $\frac{\pi}{3}$ \\
\hline
\end{tabular}


Table C10. Comparison with terrestrial habitats. Results of negative binomial GLMMs to explore how bee counts along a transect vary with habitat. All models included weather as fixed effects and observer and site as random effects to control for these variables, but we did not test for their significance. Marginal (Marg) $R^{2}$ values relate to fixed effects only, Conditional (Cond) $R^{2}$ values relate to fixed + random effects. Habitat types $(n)=$ Acid grassland (628), Arable and horticulture (122), Bog (242), Bracken (96), Broadleaved mixed and yew woodland (151), Coniferous woodland (98), Dwarf shrub heath (316), Fen, marsh and swamp (156), Improved grassland (1418), Neutral grassland (1418), Salt marsh ungrazed (104), Salt marsh extensively grazed (72), Salt marsh intensively grazed (104), Urban (166).

\begin{tabular}{|c|c|c|c|c|c|c|c|c|}
\hline \multirow[t]{2}{*}{ Model } & \multirow[t]{2}{*}{ Response } & \multirow[t]{2}{*}{$\begin{array}{l}\text { Random } \\
\text { effects }\end{array}$} & \multirow[t]{2}{*}{ Fixed effects } & \multicolumn{3}{|c|}{$\begin{array}{l}\text { Likelihood ratio test for } \\
\text { effect of habitat }\end{array}$} & \multirow{2}{*}{$\begin{array}{l}\text { Marg } \mathbf{R}^{\mathbf{2}} \\
\text { (Cond } \\
\mathbf{R}^{2} \text { ) }\end{array}$} & \multirow[t]{2}{*}{$\mathbf{n}$} \\
\hline & & & & $x^{2}$ & df & P-value & & \\
\hline 1 & $\begin{array}{l}\text { Honeybee } \\
\text { count }\end{array}$ & $\begin{array}{l}\text { Observer } \\
\text { Site/Transect }\end{array}$ & $\begin{array}{l}\text { Habitat } \\
\text { Wind } \\
\text { Temperature } \\
\text { Temperature }\end{array}$ & 101.24 & 13 & $<0.0001$ & $\begin{array}{l}0.250 \\
(0.682)\end{array}$ & 4817 \\
\hline 2 & $\begin{array}{l}\text { Bumblebee } \\
\text { count }\end{array}$ & $\begin{array}{l}\text { Observer } \\
\text { Site/Transect }\end{array}$ & $\begin{array}{l}\text { Habitat } \\
\text { Wind } \\
\text { Temperature } \\
\text { Temperature }^{3}\end{array}$ & 45.26 & 13 & $<0.0001$ & $\begin{array}{l}0.060 \\
(0.622)\end{array}$ & 4817 \\
\hline 3 & $\begin{array}{l}\text { Solitary } \\
\text { bee count }\end{array}$ & $\begin{array}{l}\text { Observer } \\
\text { Site/Transect }\end{array}$ & $\begin{array}{l}\text { Habitat } \\
\text { Wind } \\
\text { Temperature } \\
\text { Temperature }^{4}\end{array}$ & 38.98 & 13 & $<0.001$ & $\begin{array}{l}0.082 \\
(0.489)\end{array}$ & 4817 \\
\hline
\end{tabular}




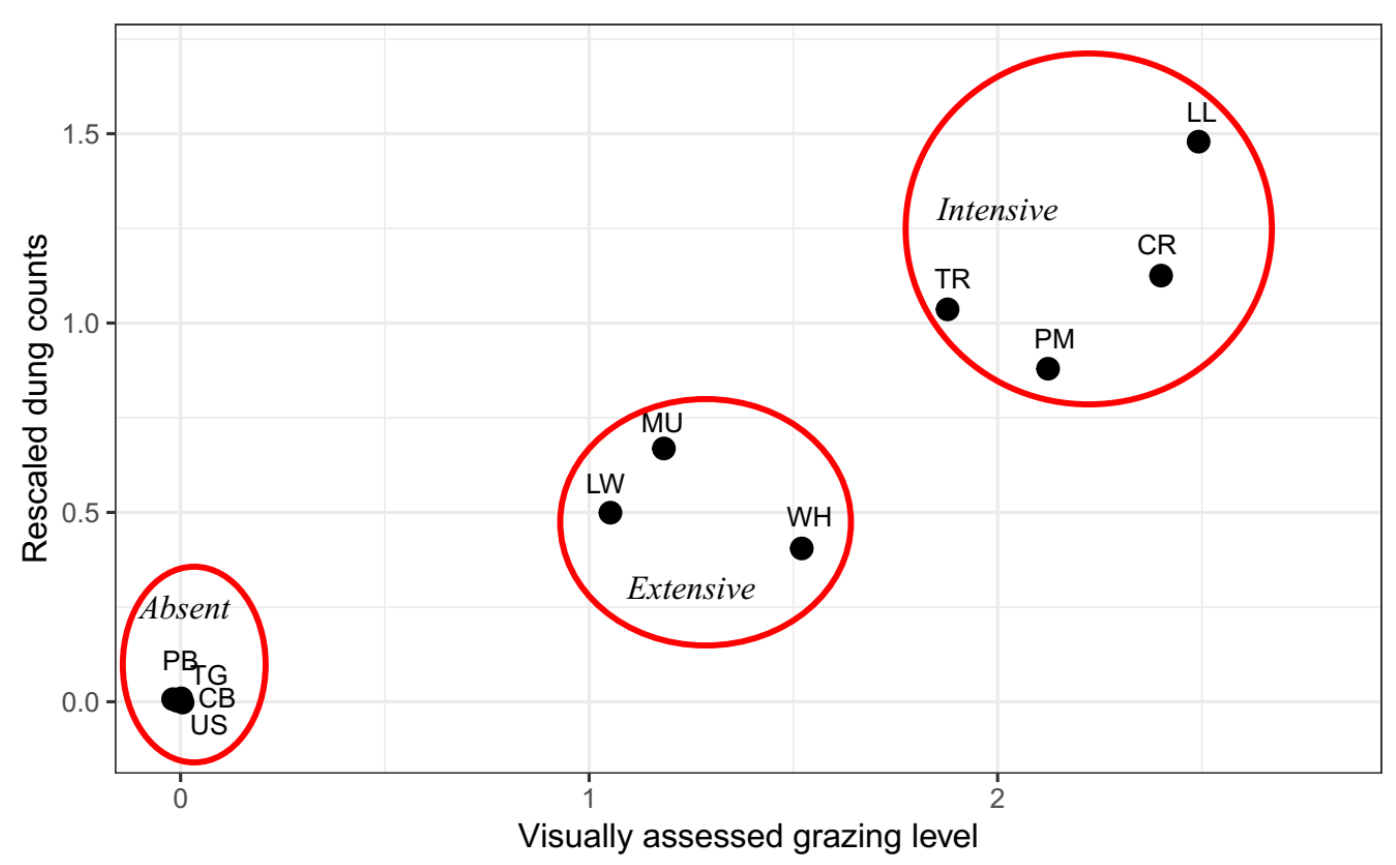

Fig. C1. Allocation of grazing levels based on dung counts and qualitative assessment.

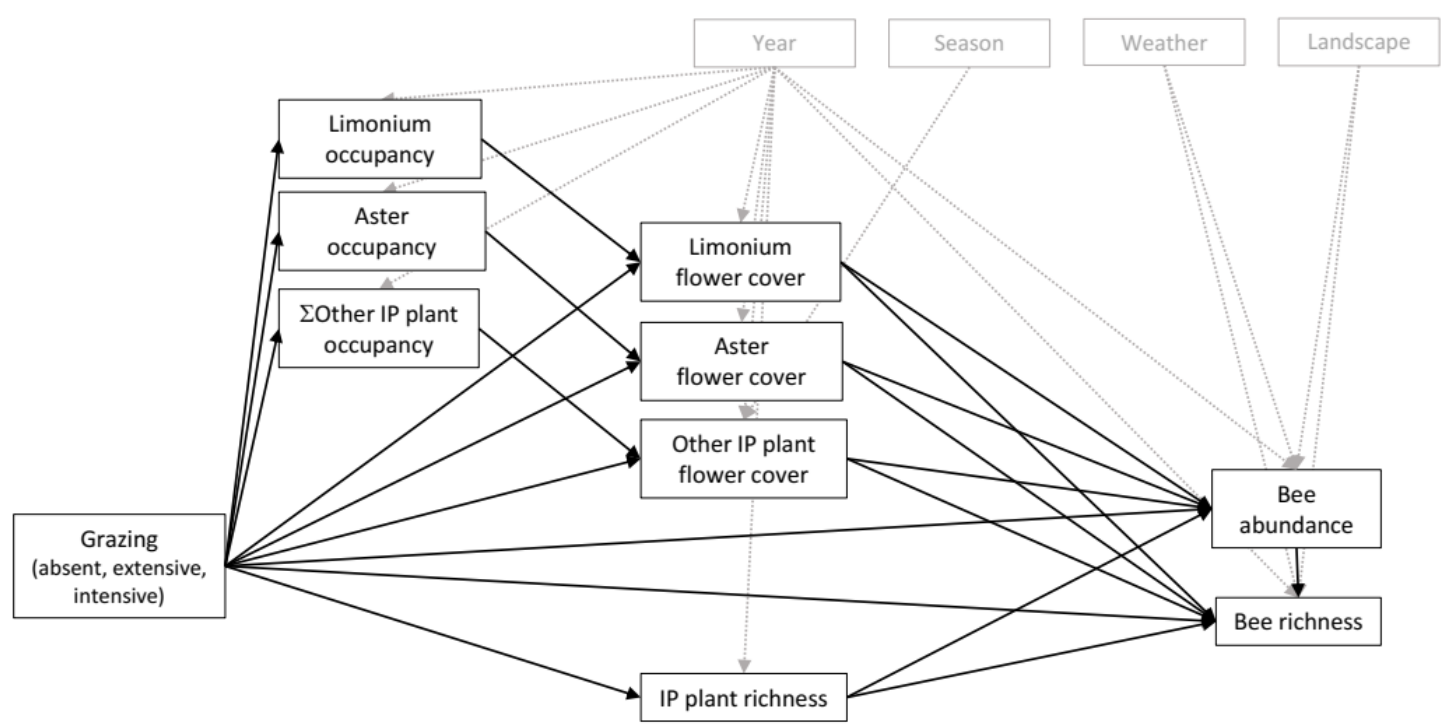

Fig. C2. Network tested using LMMs with key plant species separated. We also repeated the tests using Shannon's $\mathrm{H}^{\prime}$ index as the measure of diversity and for wild bees only (i.e. Apis mellifera excluded). Weather (wind, temperature) and landscape predictors were only included where AIC values indicated model improvement. 


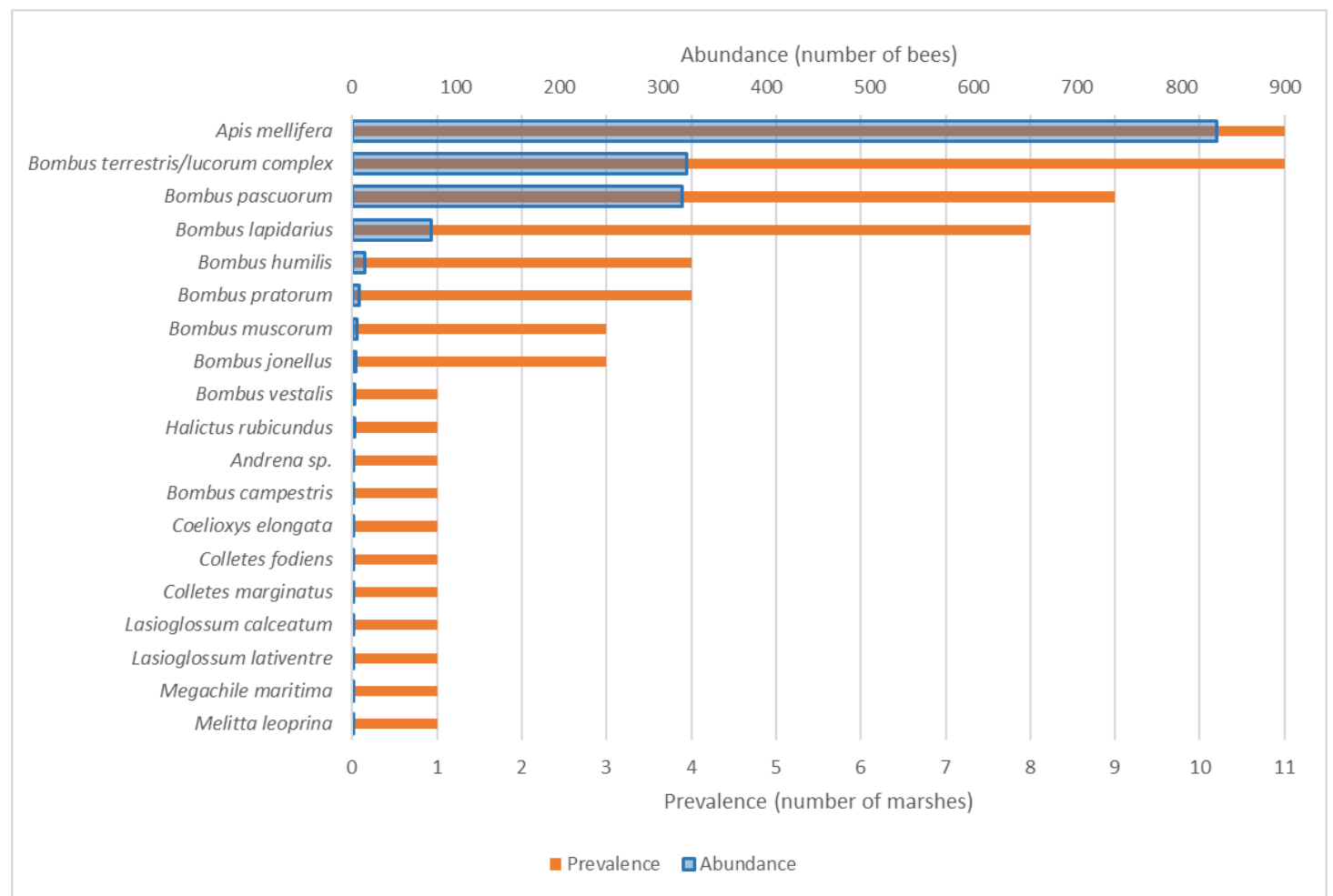

Fig. C3. Bee species by abundance and prevalence. Total number of foraging bees observed over all surveys.

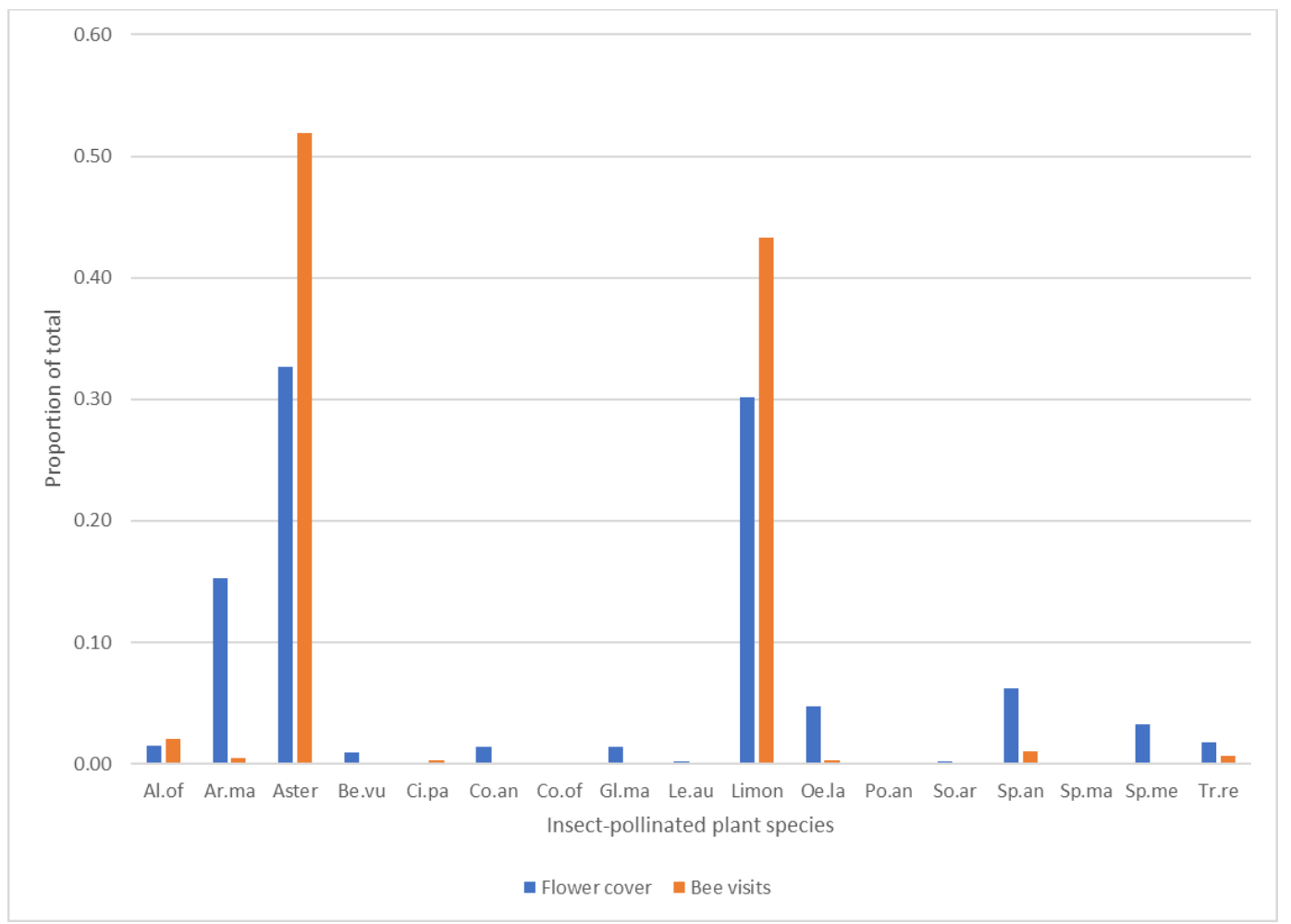

Fig. C4. Flower cover and bee visits, as a proportion of total. See Table C5 for species names of plants. 

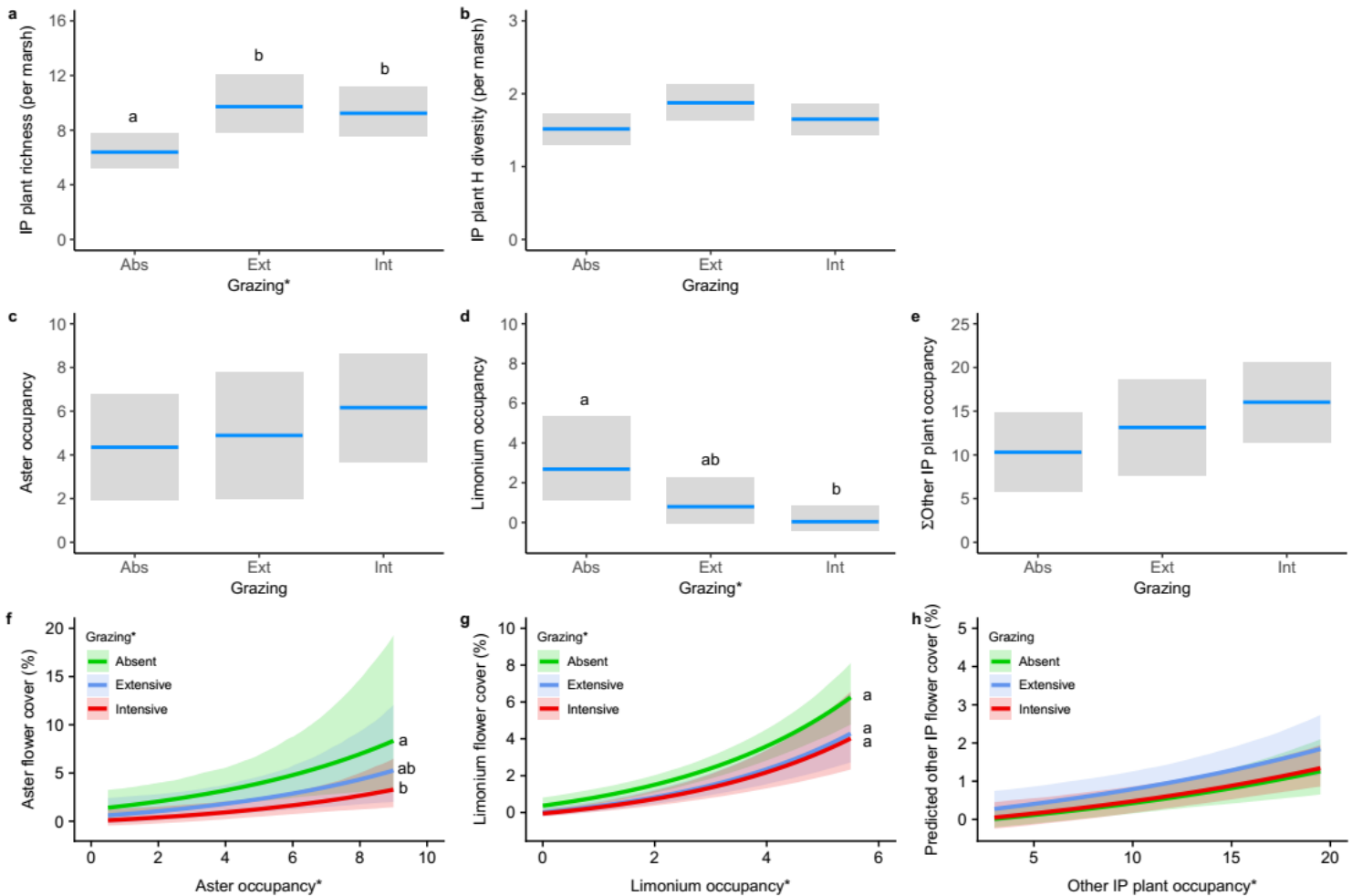

Fig. C5. Model predictions for plant responses to grazing. The effect of grazing on: insect-pollinated plant richness (a), and $\mathrm{H}$ diversity (b); the occupancy of Aster (c), Limonium (d), and other plants (e); and the flower cover of Aster (f), Limonium (g), and other plants (h). Significant predictors (likelihoodratio test) are indicated with an asterisk. Factor levels that are significantly different from each other (t-test) are indicated by different letters. 95\% confidence intervals calculated by bootstrapping. All predictions have been backtransformed to the original scale. 

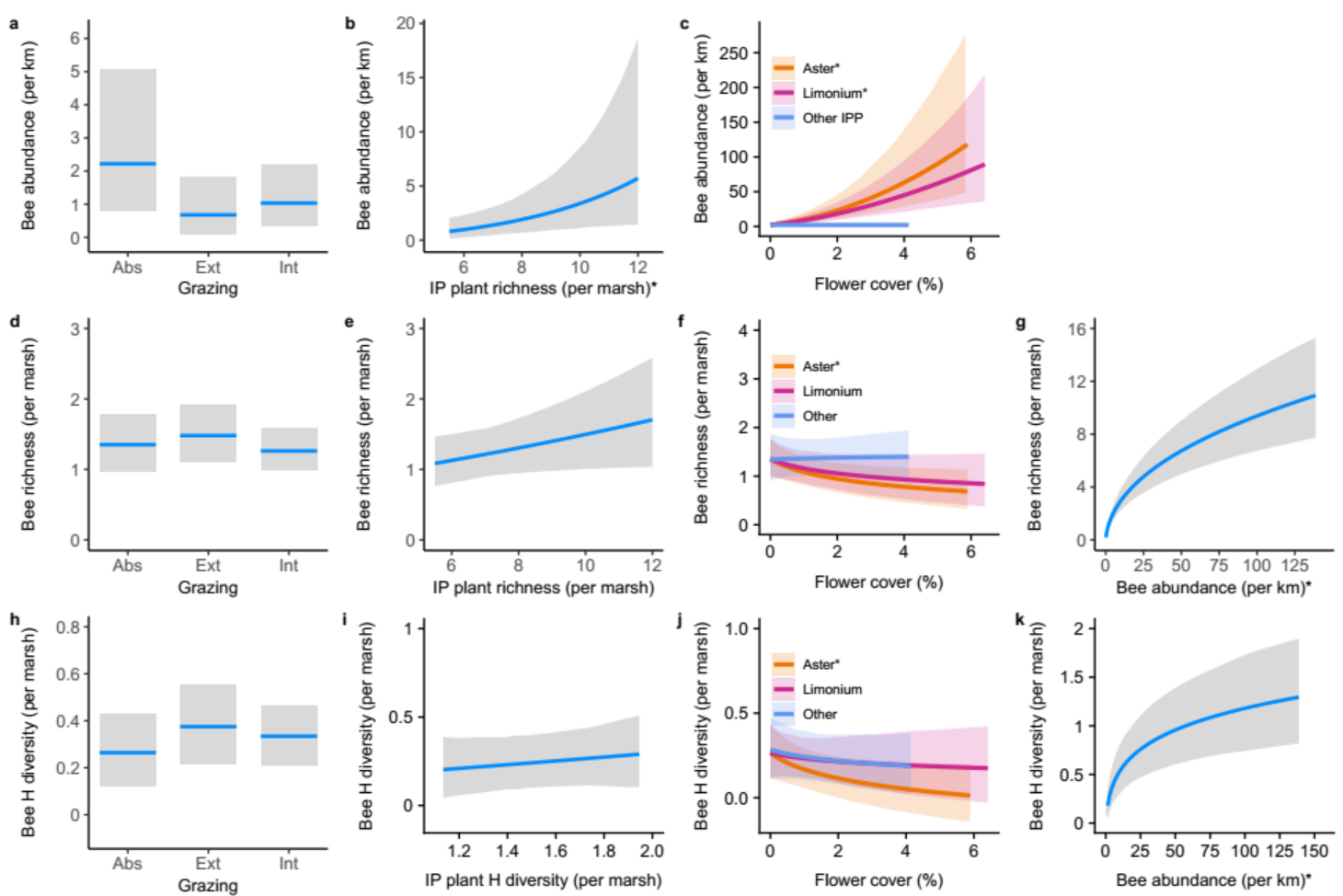

Fig. C6. Model predictions for bee responses to grazing and vegetation. The effect of grazing (a), plant richness (b), and flower cover (c) on bee abundance. The effect of grazing (d), plant richness (e), flower cover ( $f$ ), and bee abundance ( $g$ ) on bee richness. The effect of grazing (h), plant Shannon diversity (i), flower cover ( $j$ ) and bee abundance (k), on bee Shannon diversity. Significant predictors (likelihood-ratio test) are indicated with an asterisk. 95\% confidence intervals calculated by bootstrapping using the bootMer function. All predictions have been backtransformed to the original scale. 
a)
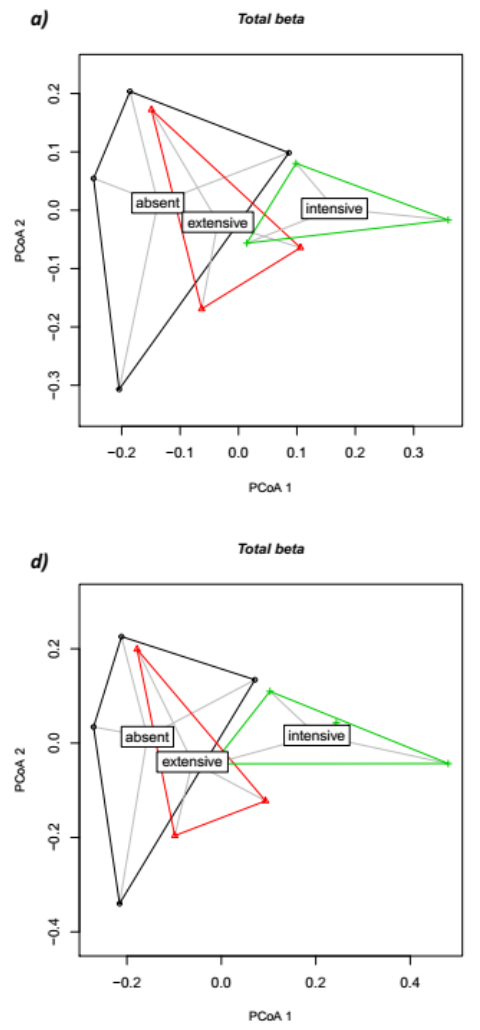
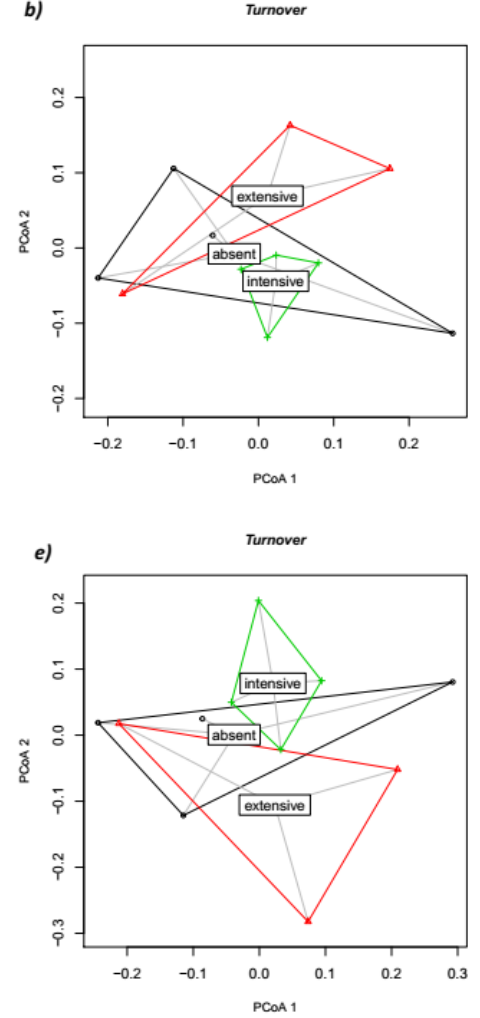

c)
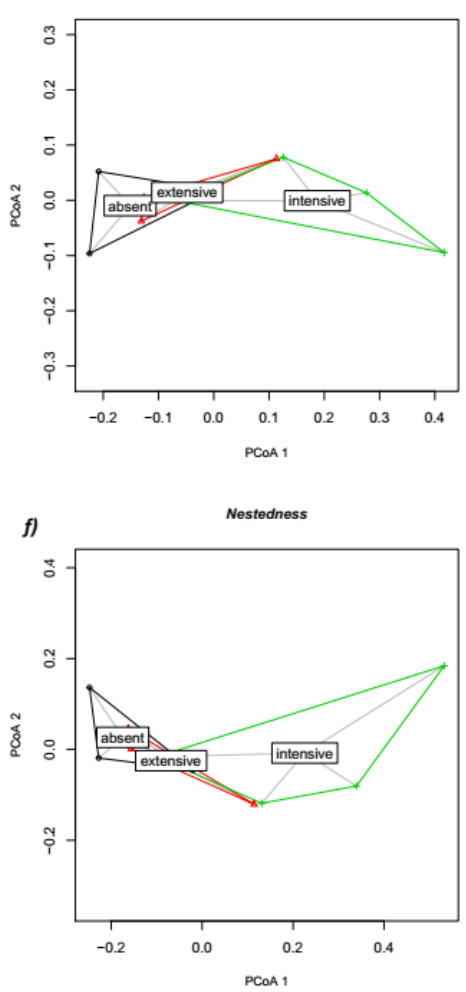

Fig. C7. $\beta$-diversity analyses. A two-dimensional MDS representation of the effect of grazing on pairwise dissimilarity of marshes for all bee species $(a-c)$ and wild bee species $(d-f)$. Pairwise distances are shown for total $\beta$-diversity $(a, d)$, the turnover partition $(b, e)$ and the nestedness partition $(c, f)$. 


\section{Appendix D: Supporting information for Chapter 5}

\section{Contents}

Supplementary methods. Calculating Ecosystem Service values

Table D1. Linear model results for optimal predictive model of bee habitat value.

Table D2. Predicted carbon stock in top $10 \mathrm{~cm}$ of saltmarsh sediment by NVC class.

Table D3. Features contributing to overall birdwatching value.

Table D4. Marsh attributes and raw ecosystem service (ES) values for all assessed marshes.

Table D5. Rescaled ecosystem service values, multifunctionality scores and ES bundles for all marshes.

Fig. D1. Heat maps of individual ES provision.

Fig. D2. Confidence in each ES map layer.

Fig. D3. Confidence levels for ES multifunctionality values and ES bundles. 200 


\section{Supplementary methods - Calculating Ecosystem Service values}

\section{D1 Plant diversity}

The Plant diversity map layer displays the average plant richness for each marsh. Water Framework Directive (WFD) sampling data from 2013 and 2015 were obtained from Natural Resources Wales (NRW) and used to calculate the average number of plant species per transect for each marsh. For WFD field surveys, transects are run from landward to seaward extent approximately every 500-1000m along a marsh. Species composition (species present and percentage cover) and sward height (five measures per transect) is recorded in two $2 \times 2 \mathrm{~m}$ quadrats at stations along the transect, with stations positioned to sample all major communities within the marsh. As well as taxa recorded in each quadrat, any additional species noted along the transect are recorded. Each WFD transect was allocated to a marsh based on the location of its landward GPS co-ordinate. Some of the smaller marshes had only one transect per marsh, whereas larger marshes had up to six. We would expect to see more species when more transects have been surveyed, therefore the plant diversity measure was calculated as the average number of species per transect for each marsh. Plant diversity was rescaled from $0-100 \%$ with $0 \%$ set as the minimum number of species recorded on any transect (10) and $100 \%$ set as the maximum value recorded within the study area (34.5).

\section{Limitations and confidence levels}

For WFD surveys, each transect is assessed in two quadrats within each zone. Although additional species observed along the transect are noted, these can be easily missed, especially in heavily grazed marshes where the sward is short and some species become miniaturised. Diversity levels may therefore be underestimated in intensively-grazed marshes. The survey effort was consistent across all surveyed marshes i.e. surveyed from landward to seaward extent every $500-100 \mathrm{~m}$ by trained field ecologists. For this reason, I have allocated all surveyed marshes a high confidence level.

\section{D2 Bird habitat}

The Bird habitat layer displays the habitat value for overwintering birds, based on Wetland Bird Survey (WeBS) data. The habitat value is calculated from the number of regularly occurring, saltmarsh-dependent, overwintering bird species present, and the UK conservation status of those species. Historical WeBS Core Count data for 18 survey sectors covering marshes in the focal area were obtained from NRW. WeBS is a long-term monitoring scheme for non-breeding waterbirds in the UK co-ordinated by the British Trust for Ornithology (BTO). Volunteer surveyors conduct synchronised 
monthly counts at wetlands, mainly during the winter months. Only resident and winter bird species (defined by RSPB 2018) were included here, as some marshes are only surveyed during winter months. Including summer birds would therefore strongly skew the results towards marshes that are surveyed during more months throughout the year. WeBS survey sectors cover many habitat types, including salt marsh, mudflat, sand dune and beach. To isolate the effect of the salt marsh, I only considered bird species that are mainly saltmarsh users. These species were identified through consultation with NRW ornithologists and included all geese and swan species, little egret, grey heron, all plovers and lapwing, teal, pintail, mallard, wigeon, pochard and gadwall (22 species in total). A habitat score for overwintering saltmarsh birds was calculated as follows. I examined the 10 years of data from $2007 / 8$ to $2016 / 17$. Years surveyed $<3$ months were discounted. Each species was given a weighting based on its UK conservation status under the Red List for Birds (Eaton et al. 2015); Red species = 3, Amber species = 2, Green or Introduced species $=1$. Pintail were given an additional point on their weighting in Burry Inlet sites, as they are a feature of Burry Inlet SPA. Each species was only counted if it was present on the marsh regularly, i.e. noted as present for $>30 \%$ of the years that sector was surveyed. The scores were then totalled for that sector. For example, a sector that had 3 green bird species, 6 amber bird species, and 1 red bird species noted as present during at least $30 \%$ of the survey years would be given a conservation score of: $\quad 3 \times 1+6 \times 2+1 \times 3=18$

The sector score was then rescaled between $0-100 \%$, with $0 \%$ set as a conservation score of 0 and $100 \%$ set as the maximum score obtained in the study area (29). Generally, a single WeBS sector covers multiple marshes, and each marsh falling within the sector was allocated the habitat value of the sector. When a marsh was overlapped by two or more WeBS sectors, it was allocated the average habitat value across the represented sectors.

\section{Limitations and confidence levels}

Each WeBS sector received varying levels of survey effort, which may affect the results. I have attempted to control for this by only using data from years surveyed $\geq 3$ months, only investigating overwintering birds (winter is the core survey period), and only assessing species diversity rather than species abundance (which would be more heavily influenced by the number of months surveyed). One may expect to see more species in larger sectors, but there was no correlation between marsh size and bird habitat value, so we were unable to correct for this. Not all marshes are covered by a WeBS sector. In particular, most marshes on the River Taf are unrepresented. Each WeBS sector was given a confidence level based on the survey effort within that sector. For each sector, years that were surveyed through 10 or more months were given a weighting of 3, years surveyed through 6-9 months were given a weighting of 2 , years that were surveyed through 3-5 months were given a weighting of 1 , and 
years surveyed for 0 - 2 months were given a weighting of 0 . This produced a theoretical overall confidence rating of between 0 (a sector surveyed for $0-2$ months per year for 10 years) and 30 (a sector surveyed for over 10 months per year over 10 years). Confidence levels were defined as: 0-10 = low, 11-20 = medium, 21-30 = high.

\section{D3 Bee habitat}

The Bee habitat layer displays the predicted bee abundance per km of transect, based on the grazing level in each marsh (as calculated in section A1.4 below) and plant community composition (from WFD survey data). I used bee survey data collected from 11 salt marshes across South Wales in 2016-2018 (see Chapter 4 for methodology) to calculate the predicted relationship between bee abundance, grazing intensity, the occupancy of two key plants ('Aster' Tripolium pannonicum and Limonium spp.), and plant species richness. I used linear mixed models (with marsh as a random effect to control for repeated sampling) to test the predictive capacity of these variables, with interactions between variables included and excluded, to produce an optimum predictive model (based on $\triangle \mathrm{AIC}$ ) using grazing and plant community data that are available across the study area. For the 11 surveyed salt marshes, I found that grazing intensity (on a scale of 0-2 where $0=$ ungrazed, $1=$ extensively grazed, $2=$ intensively grazed), plant species richness, an interaction of season (early, mid, late summer) and Aster occupancy (proportional presence in all surveyed quadrats), and an interaction of season and Limonium occupancy explained $60 \%$ of the variance in our observed bee abundances (see Table A1 for model results). I used this model to predict bee abundance for each marsh in early, mid and late summer, then took the average of these 3 values as our bee habitat value. Grazing levels for each marsh were calculated as ungrazed, lightly, moderately and heavily grazed, as described in A1.4 below. These grazing levels were assigned values of $0,1,1.5$ and 2, respectively, for these predictive models. Plant richness and Aster and Limonium occupancy were extracted from WFD survey data (see section A1.1). The Aster and Limonium occupancy values were weighted by the relative coverage of each zone in each marsh, where:

Aster occupancy for marsh =

Aster occ. in pioneer quadrats $\mathrm{x}$ proportion of marsh that is pioneer zone

+ Aster occ. in low/mid quadrats $x$ proportion of marsh that is low/mid zone

+ Aster occ. in upper/reedbed quadrats $x$ proportion of marsh that is upper/reedbed zone.

Predicted bee habitat values were rescaled between $0-100 \%$, with $0 \%$ set as the minimum predicted value $(0)$ and $100 \%$ set as the maximum predicted value $(40.7)$ in the study area.

Limitations and confidence levels 
The allocated bee habitat values are based on model predictions following field surveys within a subsample of marshes. The model explains $60 \%$ of variance in the observed bee abundances, but has not been tested with new data. The accuracy of the predictions depends on the accuracy of the vegetation data and the accuracy of the grazing levels. Vegetation monitoring effort was consistent across the survey site, therefore confidence levels for bee habitat values were the same as the confidence levels assigned for grazing levels (described in A1.4 below).

Table D1 Linear model results for optimal predictive model pf bee habitat value. Response is log (bee abundance +1$)$. Predictions were back-transformed to the original density scale for mapping. 'Marsh' was included as a random effect. Marginal $r^{2}=0.604$, conditional $r^{2}=0.604$.

\begin{tabular}{|llllll|}
\hline & Estimate & Std. Error & df & t & $\mathrm{P}$ \\
\hline (Intercept) & 0.953 & 0.953 & 76 & 1.000 & 0.320 \\
\hline Grazing intensity & -0.867 & 0.209 & 76 & -4.158 & $<0.0001$ \\
\hline Plant richness & 0.151 & 0.082 & 76 & 1.842 & 0.069 \\
\hline Early summer:Aster occ. & -1.246 & 0.872 & 76 & -1.429 & 0.157 \\
\hline Mid summer:Aster occ. & 0.708 & 0.836 & 76 & 0.848 & 0.399 \\
\hline Late summer:Aster occ. & 2.363 & 0.872 & 76 & 2.709 & 0.008 \\
\hline Early summer:Limonium occ. & -3.663 & 1.509 & 76 & -2.427 & 0.018 \\
\hline Mid summer:Limonium occ. & 4.782 & 1.386 & 76 & 3.450 & $<0.001$ \\
\hline Late summer:Limonium occ. & -1.154 & 1.509 & 76 & -0.765 & 0.447 \\
\hline
\end{tabular}

\section{D4 Livestock grazing}

The Grazing layer displays the estimated grazing intensity on each marsh. Currently, there is no accurate information on livestock grazing levels in these marshes. Grazing levels can fluctuate strongly throughout the year and from year to year. Additionally, grazing intensity is not evenly distributed across a marsh as livestock favour higher, drier ground (Sharps et al. 2017), avoid grazing some types of vegetation (e.g. Juncus maritimus) and large areas of marsh can be totally inaccessible due to large creeks. Due to these complications, I have used 3 sources of information to determine grazing intensity: observations of grazing activity from Carmarthen Bay \& Estuaries Estuarine Marine Site (CBEEMS) condition monitoring in 2012, sward height and descriptions from WFD surveys in 2013/2015, and self-reported grazing levels from owner/occupier interviews in 2017.

\section{Indicator 1: CBEEMS survey (2012) descriptions of grazing levels}

Saltmarsh surveys were conducted in 2012 by NRW for CBEEMS monitoring, and the grazing level at each survey point was noted. From these data I calculated, for each marsh, the proportion of survey points that were marked as ungrazed, lightly grazed, moderately grazed and heavily grazed. I then calculated a weighted average grazing intensity between 0 (entirely ungrazed) and 1 (entirely heavily grazed) as: CBEEMS grazing level $=(($ proportion ungrazed $x 0)+($ proportion lightly grazed $\times 1)+$ (proportion moderately grazed $\times 2)+($ proportion heavily grazed $\times 3)) / 3$. 
Indicator 2: WFD survey (2013 \& 2015) sward height data and descriptions of grazing levels

From WFD 2013/5 data I calculated the average sward height for each zone within each marsh and plotted this onto an adapted saltmarsh zonation shapefile (WFD Cycle 3 zonation shapefile provided by NRW). Some quadrats within the WFD plant survey data were assigned a zone description of 'mixed'. For these quadrats, I examined the species composition and marsh position to allocate those height data to the most appropriate zone. I then calculated an overall average sward height (weighted by the area of each zone) for each marsh. Vegetation height can be used as an indicator of grazing activity, as it is negatively and linearly correlated with livestock density (Chapter 2). However, sward height is also affected by vegetation composition, marsh zonation, and grazing by wild animals, so this measure cannot be used as a direct proxy of livestock grazing intensity. I considered that an overall average marsh sward height of $<15 \mathrm{~cm}$, or the two shortest quadrats having average sward heights $<2 \mathrm{~cm}$ was indicative of heavy grazing. I considered that an overall average marsh sward height of $<25 \mathrm{~cm}$, or the two shortest quadrats having average sward heights height $<8 \mathrm{~cm}$ to be indicative of moderate grazing. I also examined descriptions of grazing levels for each quadrat.

Indicator 3: Owner/occupier interviews (2017)

Questionnaire-based interviews of saltmarsh owner/occupiers were conducted in December 2017 as part of a collaborative research project into Welsh salt marshes by the Resilcoast research cluster (http://www.nrn-Icee.ac.uk/resilcoast/). I contacted owner/occupiers of 28 of the largest marshes in the study area by telephone and email, and received responses from 19. These interviews were undertaken in accordance with the Research Ethics Guidelines of Cardiff University and approved by the Research Ethics Committee of the School of Earth and Ocean Sciences. The questionnaires covered current and historical grazing practices, as well as areas of farm income and subsidies, and any other uses or benefits of the salt marshes. The questionnaire and summarised results are presented in Appendix E. From these data I calculated the stocking rate in livestock units (LSU) per hectare per year for each marsh subsection. LSUs were calculated as cattle $=1 \mathrm{LSU}$, young cattle $=0.6$, sheep $=0.12$, pony=0.5 (Welsh Government 2013b).

\section{Calculation of overall grazing level}

Using the CBEEMS grazing scores, I categorised each marsh as $0=$ ungrazed, 0.01 $0.25=$ lightly grazed $0.26-0.49=$ moderately grazed, $>0.50=$ heavily grazed. I then examined the WFD data to determine whether there was good reason to change the 
CBEEMS grazing levels based on these more recent surveys. Where CBEEMS or WFD data were inconsistent or absent, I examined interview data to help allocate a grazing level. Marshes were never allocated a grazing level based solely on interview data. Grazing intensity levels were rescaled from $0-100 \%$ as: ungrazed $=0 \%$, lightly grazed $=30 \%$, moderately grazed $=60 \%$, heavily grazed $=100 \%$.

\section{Limitations and confidence levels}

Grazing levels can change from year to year and season to season. Therefore, the information presented here is temporally-limited based on when surveys were conducted. Descriptions in CBEEMS and WFD surveys are subjective and depend on the area where quadrats were located. Final decisions on grazing levels were also subjective, although confidence levels were allocated to demonstrate the weight of evidence supporting that grazing level. The grazing levels are presented at the marsh scale. In reality, grazing levels will be highly variable within each marsh, with grazers concentrated around watering holes and drier areas, avoiding areas with unfavoured vegetation (e.g. Juncus maritimus, Atriplex portulacoides) and completely absent from inaccessible or very wet areas. Grazing by wild animals (e.g. geese, rabbits) is not considered here, although it may have an effect on indicators such as sward height. Where at least 2 indicators were in agreement, we allocated a high confidence rating. Marshes were allocated a medium confidence rating where there was only 1 indicator, or where WFD grazing levels differed from CBEEMS grazing levels. Marshes were given a low confidence rating where the only indicator was WFD data and grazing levels were borderline (best guess taken) or no grazing was noted (assumed to be ungrazed).

\section{D5 Carbon storage}

The Carbon storage layer presents the predicted carbon stock (tonnes of carbon per ha of marsh) in the top $10 \mathrm{~cm}$ of saltmarsh sediment. Carbon stock is predicted by soil type and plant community (Table A2) using field survey data collected by Bangor and Swansea Universities. Predictions for National Vegetation Classification types SM13, SM14, SM15, SM16 and SM18 are based on data collected in 23 Welsh saltmarshes by Bangor University in July 2015 (see Skov et al. 2016 for full methods). Each saltmarsh was sampled in 1-4 NVC types, depending on availability at the site. Four $1 \times 1 \mathrm{~m}$ quadrats were sampled per NVC type. In each quadrat, a soil sample from the top $10 \mathrm{~cm}$ was removed to test for bulk density, organic matter content and grain size. Soil texture was determined in the field using the British Columbia 'Estimating soil texture in the field' protocol (https://www.for.gov.bc.ca/isb/forms/lib/fs238.pdf). Organic matter content in the soil was estimated using loss-on-ignition $\left(375^{\circ} \mathrm{C}, 16 \mathrm{~h}\right)$ and was converted to soil 
organic carbon content using a conversion factor of 0.55 (Emmett et al. 2010).

Carbon content was converted to carbon density:

Carbon density $\left(\mathrm{gC} \mathrm{cm}^{-3}\right)=$ carbon content $\left(\mathrm{g} \mathrm{C} 100 \mathrm{~g}^{-1}\right.$ soil $) \div 100 \times$ bulk density (g soil cm ${ }^{-3}$ )

Then to tonnes carbon in the top $10 \mathrm{~cm}$ soil per hectare:

Carbon stock $\left(\mathrm{tC} \mathrm{ha}^{-1}\right)=$ Carbon density $\left(\mathrm{gC} \mathrm{cm}^{-3}\right) \times 1000$

A mixed effects model was used to predict soil carbon using NVC type and soil type (sandy or clay-loam). This model accounts for $44 \%$ of the observed variation in soil carbon. Currently, no reliable maps of saltmarsh soil types exist. However, existing field measures (Skov et al. 2016) indicate that all soil types in the study area fall within clay-loam soil types, or a mix of sand and clay-loam. Therefore I have assumed a soil type of clay-loam for all sites when making predictions.

Predictions for SM6 are based on soil carbon data collected across the study area by Swansea University in 2017 (D. de Battisti, unpublished data). Six marshes within the study area were sampled in two vegetation types - Spartina anglica and Atriplex portulacoides. Each vegetation type was sampled 7 times per marsh following the methods described above. Using dominant vegetation type accounts for $37 \%$ of the observed variation in soil carbon.

Table D2. Predicted carbon stock in top $10 \mathrm{~cm}$ of saltmarsh sediment by NVC class.

\begin{tabular}{|lllll|}
\hline NVC class & Dominant species & $\begin{array}{l}\text { C stock } \\
\left(\mathrm{t} \mathrm{ha}^{-1}\right)\end{array}$ & $\begin{array}{l}\text { St.error } \\
\left(\mathrm{t} \mathrm{ha}^{-1}\right)\end{array}$ & Source \\
\hline SM6 & Spartina anglica & 26 & 1.7 & $\begin{array}{l}\text { D. de Battisti, Swansea University, } \\
\text { unpublished data }\end{array}$ \\
\hline SM13 & Puccinellia maritima & 40 & 3.3 & Skov et al. 2016 \\
\hline SM14 & Atriplex portulacoides & 33 & 3.7 & Skov et al. 2016 \\
\hline SM15 & $\begin{array}{l}\text { Juncus maritimus-Triglochin } \\
\text { maritima }\end{array}$ & 43 & 5.2 & Skov et al. 2016 \\
\hline SM16 & Juncus gerardii & 50 & 3.4 & Skov et al. 2016 \\
\hline SM18 & Juncus maritimus & 50 & 3.3 & Skov et al. 2016 \\
\hline
\end{tabular}

I estimated the carbon stock per marsh as follows. Using NVC maps from 1998/1999 surveys I plotted the carbon stock as measured in the field for all marshes that were sampled during the survey (covering $41 \%$ of total marsh area). For marshes not sampled in the field, we plotted the predicted carbon stock for that NVC class (37\% of total marsh area). Any marsh area with unknown NVC class, or with a class not listed in Table 2 ( $22 \%$ of study area) was estimated as having the average $\mathrm{C}$ stock value for the study area $\left(40 \mathrm{tC} \mathrm{ha}^{-1}\right)$. Finally, I calculated the total carbon stock in each marsh (tonnes) and divided by the area of the marsh, to obtain the average carbon stock per marsh (tonnes $\mathrm{Cha}^{-1}$ ). This value was then rescaled between 0$100 \%$, with $0 \%$ set as the minimum value recorded in the study area (22) and $100 \%$ set as the maximum value calculated for the study area (53.7). 
The carbon stocks were calculated using NVC data from 1998/1999 surveys. Although these data are out of date, surveys conducted in 2012 as part of CBE condition monitoring indicate that these communities have not changed significantly; the only major changes were found in the lower marsh Spartina zone. Predictions based on NVC class only account for $\sim 40 \%$ of the variation in soil carbon. Not all NVC classes have been sampled for carbon stock, so approximately one fifth of the saltmarsh area has been allocated an average carbon value. I have assumed a clay-loam soil type for the estuary, based on field surveys, but this is likely to vary within each marsh. Confidence levels are based on the source of carbon data, weighted by the coverage per marsh. A confidence score of 0 represents a marsh where all the data is based on an estimate of $40 \mathrm{tC} \mathrm{ha}^{-1}$ (in this situation, the carbon value was mapped as 'no data'). A confidence score of 50 represents a marsh where all the $C$ data is predicted based on NVC class. A confidence score of 100 represents a marsh where all the $C$ data is based on field measurement. A marsh with a confidence level of 0 was mapped with an ES value of 'no data'. Confidence scores below 33 are considered low, scores between 33 and 66 are considered medium and scores above 66 are considered high.

\section{D6 Birdwatching}

The Birdwatching layer shows the value attributed to each marsh for winter birdwatching. The values are informed by bird diversity and abundance, as well as other features of the marsh that may improve the birdwatching experience. I sought expert opinion from staff members in relevant public, academic and conservation bodies in Wales to determine what biotic and abiotic features of a marsh are most important for birdwatchers. I obtained responses from the Royal Society for the Protection of Birds ( $n=1)$, the Wildfowl and Wetland Trust (1), the British Trust for Ornithology (1), the Wildlife Trust of South and West Wales (3), Cardiff University (1) and NRW (2). The questionnaire and summarised responses are presented in Appendix F. The features were ranked in order of importance (high to low) as bird diversity, bird abundance, car parking, easy access, presence of a bird hide, proximity to home, presence of a particular species, other feature. Under 'other feature', low human disturbance was stated by 3 respondents and toilets/facilities was stated by 2 respondents. I also asked respondents to rate each of the possible bird sightings in terms of desirability, from high to low, and to state any preferred species, but there was no consistency of response. Therefore, I concluded that overall bird diversity is more important than the presence of any particular species. I scored each marsh for every feature, with more highly desired features weighted more heavily (Table A3). I then summed the scores to give an overall birdwatching value, with a theoretical maximum of 100. Marshes that do not have a public right of way onto or 
immediately adjacent to the marsh were given an overall score of 0 , irrespective of other features. As birdwatching values fall in a potential range from $0-100$, I did not rescale these values.

\section{Limitations and confidence levels}

The limitations of WeBS survey data described in Section A1.2 also apply here, and the diversity and abundance values only apply to winter birds. The birdwatching score is heavily dependent on the weighting given to each feature. Most of the score (55\%) is accounted by bird diversity and abundance, which is commonly important to all bird watchers, but other features may be very important for some but not at all important to others. For example, some birdwatchers with mobility issues may put a premium on close parking, whereas others may enjoy a long walk as an intrinsic part of their birdwatching trip. The birdwatching values presented here are for the potential value, whereas an extensive survey would be required to reveal the realised value. I have allocated confidence levels based on survey effort per WeBS sector, as described in Section A1.2.

Table D3. Features contributing to overall birdwatching value. Average rank per feature, as assessed by 9 relevant experts from various public, conservation and academic institutions in Wales. Scores were weighted more heavily towards more highly ranked features, to produce a maximum total score of 100.

\begin{tabular}{|c|c|c|c|}
\hline Rank & Feature & Measure of feature & $\begin{array}{l}\text { Max. } \\
\text { score }\end{array}$ \\
\hline 1 & Bird diversity & $\begin{array}{l}\text { Average winter saltmarsh-specialist bird diversity per year over previous } 10 \\
\text { years* (rescaled from 0-30 with } 30 \text { being maximum recorded diversity). }\end{array}$ & 30 \\
\hline 2 & Bird abundance & $\begin{array}{l}\text { Average winter saltmarsh-specialist bird abundance per year over previous } 10 \\
\text { years* (rescaled from 0-25 with } 25 \text { being maximum recorded abundance). }\end{array}$ & 25 \\
\hline 3 & Car parking & \multirow{2}{*}{$\begin{array}{l}\text { Car parking \& easy access measured as walking distance to nearest car-parking } \\
\text { spot (rescaled out of } 15 \text { with maximum score being a } 0 \mathrm{~km} \text { walk) + walking } \\
\text { distance to nearest bus stop (rescaled out of } 5 \text { with maximum score being } 0 \mathrm{~km} \\
\text { walk). }\end{array}$} & \multirow[t]{2}{*}{20} \\
\hline 4 & Easy access & & \\
\hline 5 & Bird hide present & Presence of bird hide overlooking marsh (present=15, absent=0). & 15 \\
\hline 6 & Proximity to home & Not included in assessment. & NA \\
\hline 7 & $\begin{array}{l}\text { Presence of } \\
\text { particular species }\end{array}$ & $\begin{array}{l}\text { Not included as the only species specified by }>1 \text { respondent was bittern, which } \\
\text { is not regularly present in any marsh. }\end{array}$ & NA \\
\hline $8 a$ & $\begin{array}{l}\text { Other: low } \\
\text { disturbance }\end{array}$ & $\begin{array}{l}\text { Tranquillity rating from NRW Tranquil Areas Wales shapefile. Urban (or a road } \\
\text { directly adjacent to marsh)=0, Zone } C=6.5 \text {, Zone } B=3.5 \text {, Undisturbed=10. }\end{array}$ & 10 \\
\hline $8 b$ & $\begin{array}{l}\text { Other: toilets/ } \\
\text { facilities }\end{array}$ & $\begin{array}{l}\text { Not included as none of the study marshes have public toilets in immediate } \\
\text { vicinity. }\end{array}$ & NA \\
\hline \multicolumn{3}{|c|}{$\begin{array}{l}\text { Sum all scores to obtain Birdwatching value } \\
\text { (Or } 0 \text { if no public right of way onto or immediately adjacent to the marsh ) }\end{array}$} & 100 \\
\hline
\end{tabular}




\section{D7 Wildfowling}

The Wildfowling layer shows the average number of wildfowl shot per $\mathrm{km}$ of foreshore per year. Shooting grounds were drawn using maps available in Shooting and Conservation Management Plans 2011/2 - 2026/7 for the relevant associations, provided by NRW. Wildfowling bag counts were obtained from Carmarthenshire Wildfowlers Association, Llanybri Wildfowling and Conservation Association and Morlais Valley Gun Club. Numbers were averaged for the three most recent reported shooting seasons (2013/14 - 2015/16) and were standardised by the length of the shooting ground in km (excluding any no-shoot refuge areas) as site length, rather than area, is the most relevant scale for wildfowlers. The number of wildfowl shot per $\mathrm{km}$ was rescaled from $0-100 \%$ with $0 \%$ set as 0 wildfowl (no shooting permitted) and $100 \%$ set as the maximum value recorded within the study area (47). Generally, a single shooting ground covers multiple marshes, and each marsh falling within the shooting ground was allocated the wildfowling value of the shooting ground. When a marsh was overlapped by two or more shooting grounds, it was allocated the average wildfowling value across the represented shooting grounds.

\section{Limitations and confidence levels}

All shooting associations are required to report their bag returns to NRW each year. For this reason, all numbers should be accurate, and all marshes have been allocated a high confidence level. However, any unlicensed shooting will not be reported here. 
Table D4. Marsh attributes and raw ecosystem service (ES) values for all assessed marshes. Under management type $\mathrm{C}=$ common land with multiple graziers, $\mathrm{PT}=$ private owner or tenant farmer, $\mathrm{CC}=$ conservation body or local council. Distance shows the distance from the centre of the marsh to the estuary mouth (i.e. where the river opens onto Carmarthen Bay). See Appendix A for ES calculation. Plant diversity = average number of species per transect; bird habitat = no. red species $\times 3$ + no. amber species $\times 2+$ no. green species $\times 1$; bee habitat $=$ predicted bee abundance per transect $\mathrm{km}$; $\mathrm{U}=$ ungrazed, $\mathrm{L}=$ lightly grazed, $\mathrm{M}=$ moderately grazed, $\mathrm{H}=$ heavily grazed; Carbon = tonnes $\mathrm{C}$ ha ${ }^{-1}$ in the top $10 \mathrm{~cm}$ of sediment; Birdwatching = potential score out of 100 based on diversity, abundance, access, facilities and tranquillity; Wildfowling = average no. birds shot per km foreshore per year.

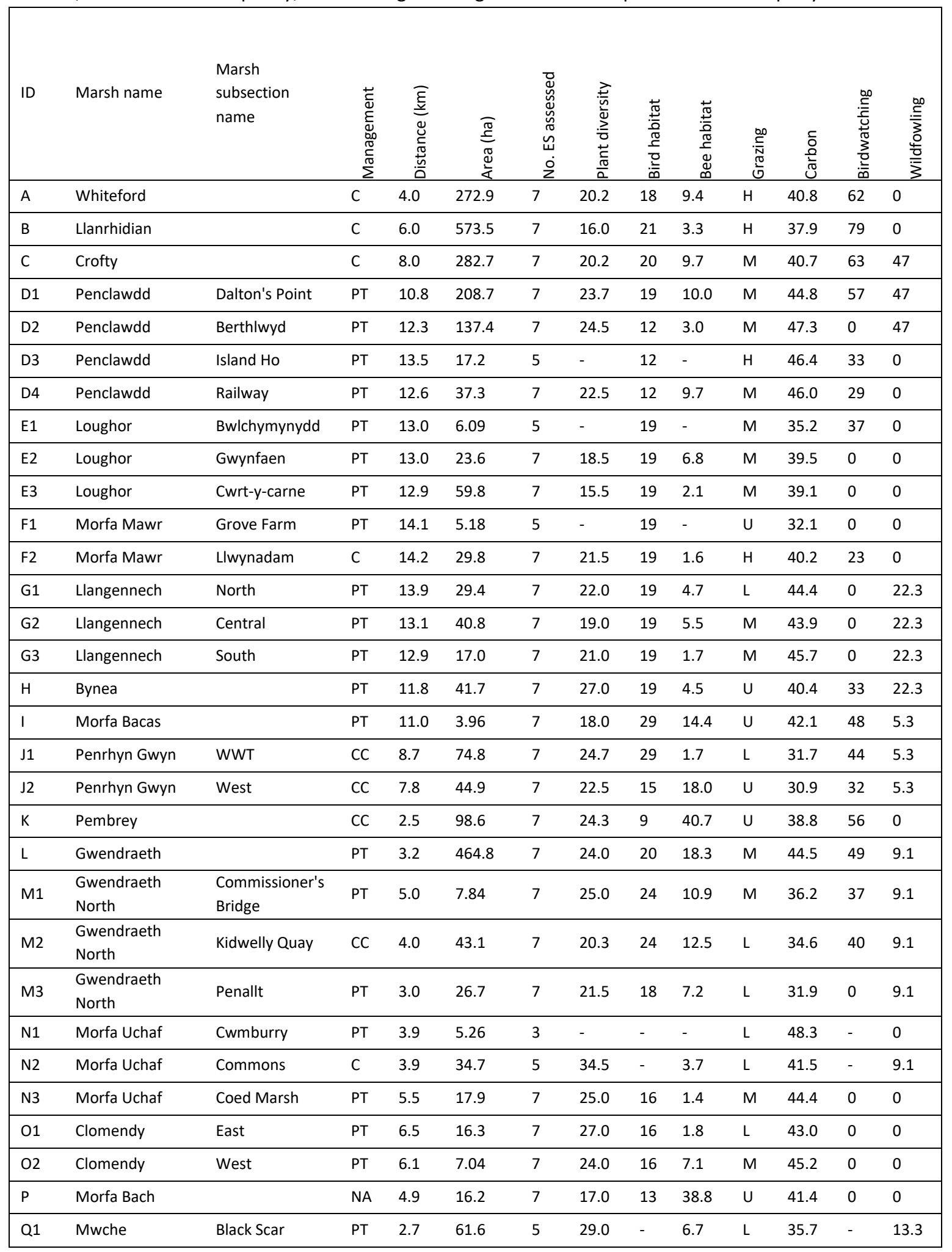




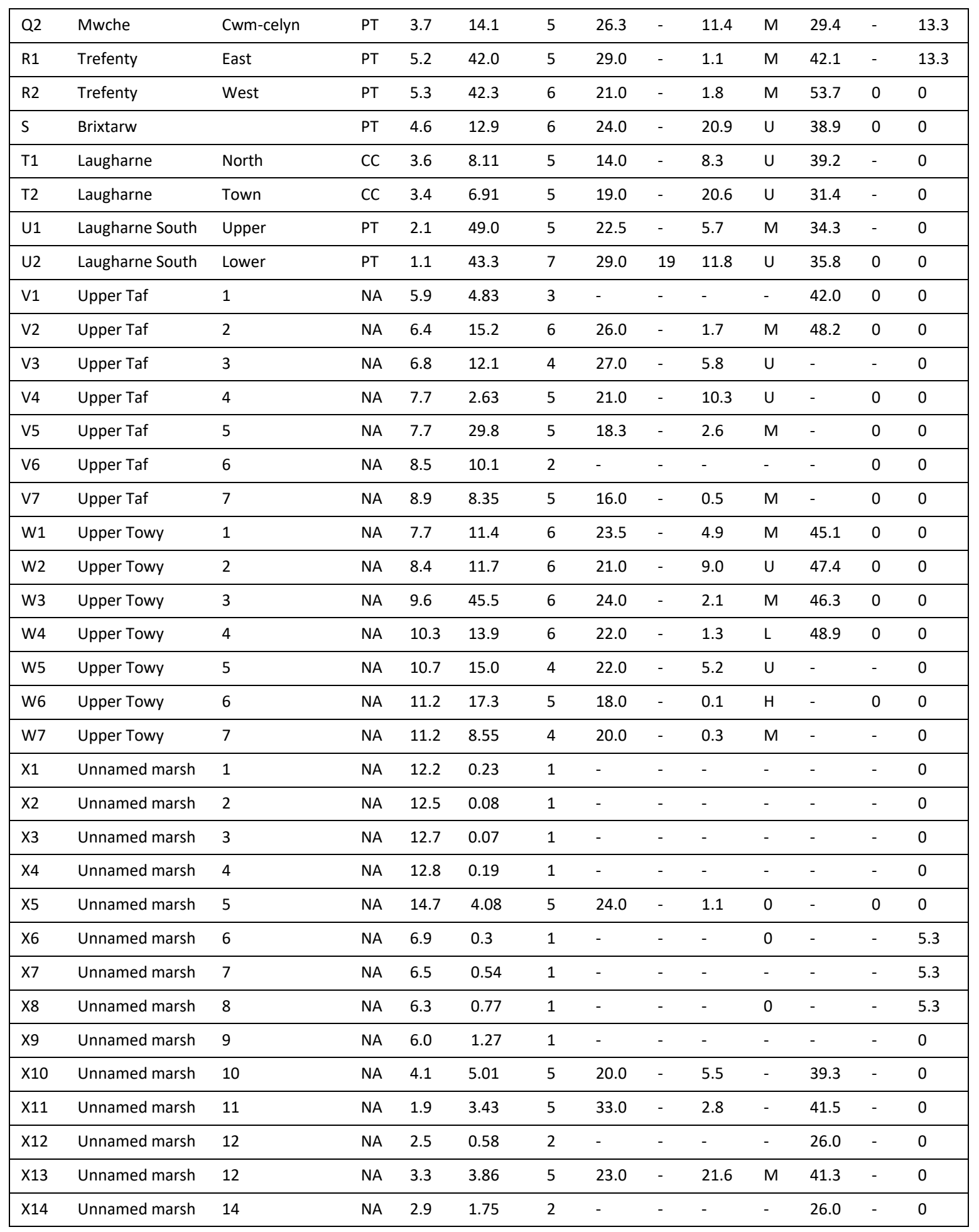


Table D5. Rescaled ecosystem service values, multifunctionality scores and ES bundles for all marshes. Values shown in brackets were estimated (as described in methods) when calculating multifunctionality scores and ES bundles to increase the sample size. ES bundles: $1=$ Grazing, no recreation, 2 = Heavy grazing \& birds, $3=$ Bee-friendly, $4=$ Biodiversity, $5=$ Multi-use, $6=$ Plant diversity. Confidence levels: 1 = low, 2 = medium, 3 = high.

\begin{tabular}{|c|c|c|c|c|c|c|c|c|c|c|c|c|c|c|}
\hline \multirow[b]{2}{*}{ ID } & \multicolumn{7}{|c|}{ Rescaled ES values } & \multicolumn{7}{|c|}{ Multifunctionality measures and ES bundles } \\
\hline & 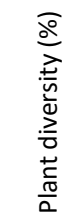 & $\begin{array}{l}\widehat{0} \\
\frac{0}{0} \\
\frac{ \pm}{0} \\
\frac{0}{0} \\
\frac{c}{0} \\
\frac{0}{0}\end{array}$ & 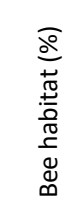 & $\begin{array}{l}\widehat{0} \\
\frac{0}{\mathfrak{N}} \\
\frac{\stackrel{0}{0}}{0}\end{array}$ & $\begin{array}{l}\widehat{o} \\
\frac{0}{0} \\
\frac{0}{2} \\
0\end{array}$ & 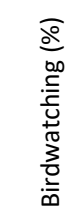 & $\begin{array}{l}\frac{\widehat{0}}{0} \\
. \stackrel{5}{3} \\
\frac{0}{3} \\
\frac{0}{0} \\
\stackrel{3}{3}\end{array}$ & 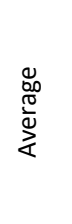 & 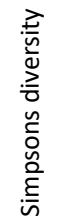 & $\begin{array}{l}\stackrel{\circ}{\circ} \\
\text { m } \\
\wedge \\
\dot{0} \\
\dot{z}\end{array}$ & $\begin{array}{l}\text { ㅇํ } \\
\text { ํ } \\
\text { ! } \\
\dot{0}\end{array}$ & $\begin{array}{l}\stackrel{\circ}{\circ} \\
\text { N1 } \\
\stackrel{0}{Z}\end{array}$ & 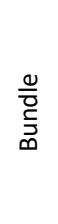 & 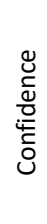 \\
\hline$A$ & 42 & 62 & 23 & 100 & 59 & 62 & 0 & 49.7 & 0.81 & 5 & 4 & 1 & 2 & 3 \\
\hline B & 24 & 71 & 8 & 100 & 50 & 79 & 0 & 47.4 & 0.78 & 4 & 4 & 3 & 2 & 3 \\
\hline C & 41 & 68 & 24 & 60 & 59 & 63 & 100 & 59.2 & 0.84 & 6 & 5 & 1 & 5 & 2 \\
\hline D1 & 56 & 66 & 25 & 60 & 72 & 57 & 100 & 62.2 & 0.84 & 6 & 6 & 2 & 5 & 3 \\
\hline D2 & 59 & 41 & 7 & 60 & 80 & 0 & 100 & 49.6 & 0.79 & 5 & 4 & 2 & 5 & 3 \\
\hline D3 & NA & 41 & NA & 100 & 77 & 33 & 0 & NA & NA & NA & NA & NA & NA & NA \\
\hline D4 & 51 & 41 & 24 & 60 & 76 & 29 & 0 & 40.0 & 0.81 & 4 & 3 & 1 & 1 & 3 \\
\hline E1 & NA & 66 & NA & 60 & 42 & 37 & 0 & NA & NA & NA & NA & NA & NA & NA \\
\hline E2 & 35 & 66 & 17 & 60 & 55 & 0 & 0 & 33.3 & 0.77 & 4 & 3 & 0 & 1 & 2 \\
\hline E3 & 22 & 66 & 5 & 60 & 54 & 0 & 0 & 29.6 & 0.73 & 3 & 3 & 0 & 1 & 3 \\
\hline F1 & NA & 66 & NA & 0 & 32 & 0 & 0 & NA & NA & NA & NA & NA & NA & NA \\
\hline F2 & 47 & 66 & 4 & 100 & 57 & 23 & 0 & 42.5 & 0.77 & 4 & 3 & 1 & 2 & 3 \\
\hline G1 & 49 & 66 & 12 & 30 & 71 & 0 & 48 & 39.3 & 0.80 & 5 & 2 & 1 & 5 & 2 \\
\hline $\mathrm{G} 2$ & 37 & 66 & 14 & 60 & 69 & 0 & 48 & 41.9 & 0.81 & 5 & 3 & 0 & 5 & 3 \\
\hline G3 & 45 & 66 & 4 & 60 & 75 & 0 & 48 & 42.6 & 0.80 & 5 & 3 & 1 & 5 & 3 \\
\hline $\mathrm{H}$ & 69 & 66 & 11 & 0 & 58 & 33 & 48 & 40.7 & 0.80 & 5 & 3 & 0 & 4 & 3 \\
\hline I & 33 & 100 & 35 & 0 & 63 & 48 & 11 & 41.6 & 0.78 & 5 & 2 & 1 & 4 & 2 \\
\hline J1 & 60 & 100 & 4 & 30 & 31 & 44 & 11 & 40.1 & 0.78 & 5 & 2 & 1 & 4 & 2 \\
\hline $\mathrm{J} 2$ & 51 & 52 & 44 & 0 & 28 & 32 & 11 & 31.1 & 0.81 & 4 & 2 & 0 & 3 & 3 \\
\hline $\mathrm{K}$ & 59 & 31 & 100 & 0 & 53 & 56 & 0 & 42.7 & 0.77 & 5 & 4 & 1 & 3 & 3 \\
\hline L & 57 & 72.5 & 45 & 60 & 71 & 49 & 19 & 53.4 & 0.84 & 6 & 4 & 2 & 2 & 2 \\
\hline M1 & 61 & 86 & 27 & 60 & 45 & 37 & 19 & 47.8 & 0.83 & 5 & 3 & 1 & 2 & 2 \\
\hline M2 & 42 & 86 & 31 & 30 & 40 & 40 & 19 & 41.0 & 0.82 & 6 & 1 & 1 & 4 & 2 \\
\hline M3 & 47 & 65.5 & 18 & 30 & 31 & 0 & 19 & 30.1 & 0.80 & 4 & 1 & 0 & 4 & 2 \\
\hline N1 & NA & (20) & NA & 30 & 83 & (48) & 0 & NA & NA & NA & NA & NA & NA & NA \\
\hline N2 & 100 & (20) & 9 & 30 & 62 & (48) & 19 & 41.0 & 0.78 & 4 & 2 & 1 & 6 & 2 \\
\hline N3 & 61 & 55 & 4 & 60 & 71 & 0 & 0 & 35.7 & 0.75 & 4 & 4 & 1 & 1 & 3 \\
\hline 01 & 69 & 55 & 4 & 30 & 66 & 0 & 0 & 32.1 & 0.74 & 4 & 3 & 0 & 1 & 2 \\
\hline $\mathrm{O} 2$ & 57 & 55 & 17 & 60 & 73 & 0 & 0 & 37.5 & 0.77 & 4 & 4 & 1 & 1 & 2 \\
\hline$P$ & 29 & 46.5 & 95 & 0 & 61 & 0 & 0 & 33.2 & 0.71 & 3 & 2 & 1 & 3 & 2 \\
\hline Q1 & 78 & (20) & 16 & 30 & 43 & (25) & 28 & 34.4 & 0.81 & 3 & 1 & 1 & 6 & 2 \\
\hline Q2 & 67 & (20) & 28 & 60 & 23 & (19) & 28 & 35.0 & 0.82 & 2 & 2 & 0 & 1 & 2 \\
\hline $\mathrm{R} 1$ & 78 & (20) & 3 & 60 & 63 & (17) & 28 & 38.4 & 0.79 & 3 & 3 & 1 & 1 & 2 \\
\hline $\mathrm{R} 2$ & 45 & (20) & 4 & 60 & 100 & 0 & 0 & 32.8 & 0.70 & 3 & 2 & 1 & 1 & 2 \\
\hline$S$ & 57 & (20) & 51 & 0 & 53 & 0 & 0 & 26.0 & 0.72 & 3 & 3 & 0 & 3 & 2 \\
\hline T1 & 16 & (20) & 20 & 0 & 54 & (38) & 0 & 21.2 & 0.75 & 2 & 1 & 0 & 6 & 2 \\
\hline
\end{tabular}




\begin{tabular}{|c|c|c|c|c|c|c|c|c|c|c|c|c|c|c|}
\hline $\mathrm{T} 2$ & 37 & (20) & 51 & 0 & 30 & (50) & 0 & 26.8 & 0.78 & 4 & 2 & 0 & 3 & 2 \\
\hline U1 & 51 & (20) & 14 & 60 & 39 & (43) & 0 & 32.3 & 0.80 & 4 & 2 & 0 & 1 & 2 \\
\hline U2 & 78 & 69 & 29 & 0 & 44 & 0 & 0 & 31.4 & 0.72 & 3 & 2 & 1 & 4 & 3 \\
\hline V1 & NA & NA & NA & NA & 63 & 0 & 0 & NA & NA & NA & NA & NA & NA & NA \\
\hline V2 & 65 & (20) & 4 & 60 & 83 & 0 & 0 & 33.1 & 0.72 & 3 & 3 & 1 & 1 & 2 \\
\hline V3 & 69 & (20) & 14 & 0 & (57) & (47) & 0 & 29.5 & 0.75 & 3 & 2 & 0 & 6 & 1 \\
\hline V4 & 45 & (20) & 25 & 0 & (57) & 0 & 0 & 21.0 & 0.71 & 2 & 1 & 0 & 6 & 1 \\
\hline V5 & 34 & (20) & 6 & 60 & (57) & 0 & 0 & 25.3 & 0.73 & 3 & 2 & 0 & 1 & 2 \\
\hline V6 & NA & NA & NA & NA & NA & 0 & 0 & NA & NA & NA & NA & NA & NA & NA \\
\hline V7 & 24 & (20) & 1 & 60 & (57) & 0 & 0 & 23.1 & 0.70 & 2 & 2 & 0 & 1 & 1 \\
\hline W1 & 55 & (20) & 12 & 60 & 73 & 0 & 0 & 31.4 & 0.74 & 3 & 3 & 1 & 1 & 2 \\
\hline W2 & 45 & (20) & 22 & 0 & 80 & 0 & 0 & 23.9 & 0.67 & 2 & 1 & 1 & 6 & 2 \\
\hline W3 & 57 & (20) & 5 & 60 & 77 & 0 & 0 & 31.3 & 0.73 & 3 & 3 & 1 & 1 & 1 \\
\hline W4 & 49 & (20) & 3 & 30 & 85 & 0 & 0 & 26.7 & 0.69 & 3 & 1 & 1 & 1 & 2 \\
\hline W5 & 49 & (20) & 13 & 0 & (57) & (42) & 0 & 25.7 & 0.76 & 3 & 1 & 0 & 6 & 1 \\
\hline W6 & 33 & (20) & 0 & 100 & (57) & 0 & 0 & 30.0 & 0.67 & 3 & 2 & 1 & 1 & 2 \\
\hline W7 & 41 & (20) & 1 & 60 & (57) & (47) & 0 & 32.2 & 0.78 & 4 & 2 & 0 & 1 & 1 \\
\hline $\mathrm{X} 1$ & NA & NA & NA & NA & NA & NA & 0 & NA & NA & NA & NA & NA & NA & NA \\
\hline$X 2$ & NA & NA & NA & NA & NA & NA & 0 & NA & NA & NA & NA & NA & NA & NA \\
\hline X3 & NA & NA & NA & NA & NA & NA & 0 & NA & NA & NA & NA & NA & NA & NA \\
\hline$X 4$ & NA & NA & NA & NA & NA & NA & 0 & NA & NA & NA & NA & NA & NA & NA \\
\hline X5 & 57 & NA & 3 & 60 & NA & 0 & 0 & 28.1 & 0.73 & 3 & 3 & 0 & 1 & 2 \\
\hline$x 6$ & NA & NA & NA & NA & NA & NA & 11 & NA & NA & NA & NA & NA & NA & NA \\
\hline X7 & NA & NA & NA & NA & NA & NA & 11 & NA & NA & NA & NA & NA & NA & NA \\
\hline$x 8$ & NA & NA & NA & NA & NA & NA & 11 & NA & NA & NA & NA & NA & NA & NA \\
\hline X9 & NA & NA & NA & NA & NA & NA & 0 & NA & NA & NA & NA & NA & NA & NA \\
\hline X10 & 41 & (20) & 14 & 0 & 55 & (30) & 0 & 22.7 & 0.76 & 3 & 1 & 0 & 6 & 1 \\
\hline X11 & 94 & (20) & 7 & 0 & 62 & (28) & 0 & 30.1 & 0.69 & 2 & 2 & 1 & 6 & 1 \\
\hline X12 & NA & NA & NA & NA & 13 & NA & 0 & NA & NA & NA & NA & NA & NA & NA \\
\hline X13 & 53 & (20) & 53 & 0 & 61 & (29) & 0 & 30.9 & 0.77 & 3 & 3 & 0 & 3 & 1 \\
\hline X14 & NA & NA & NA & NA & 13 & NA & 0 & NA & NA & NA & NA & NA & NA & NA \\
\hline
\end{tabular}


$$
\text { . }
$$
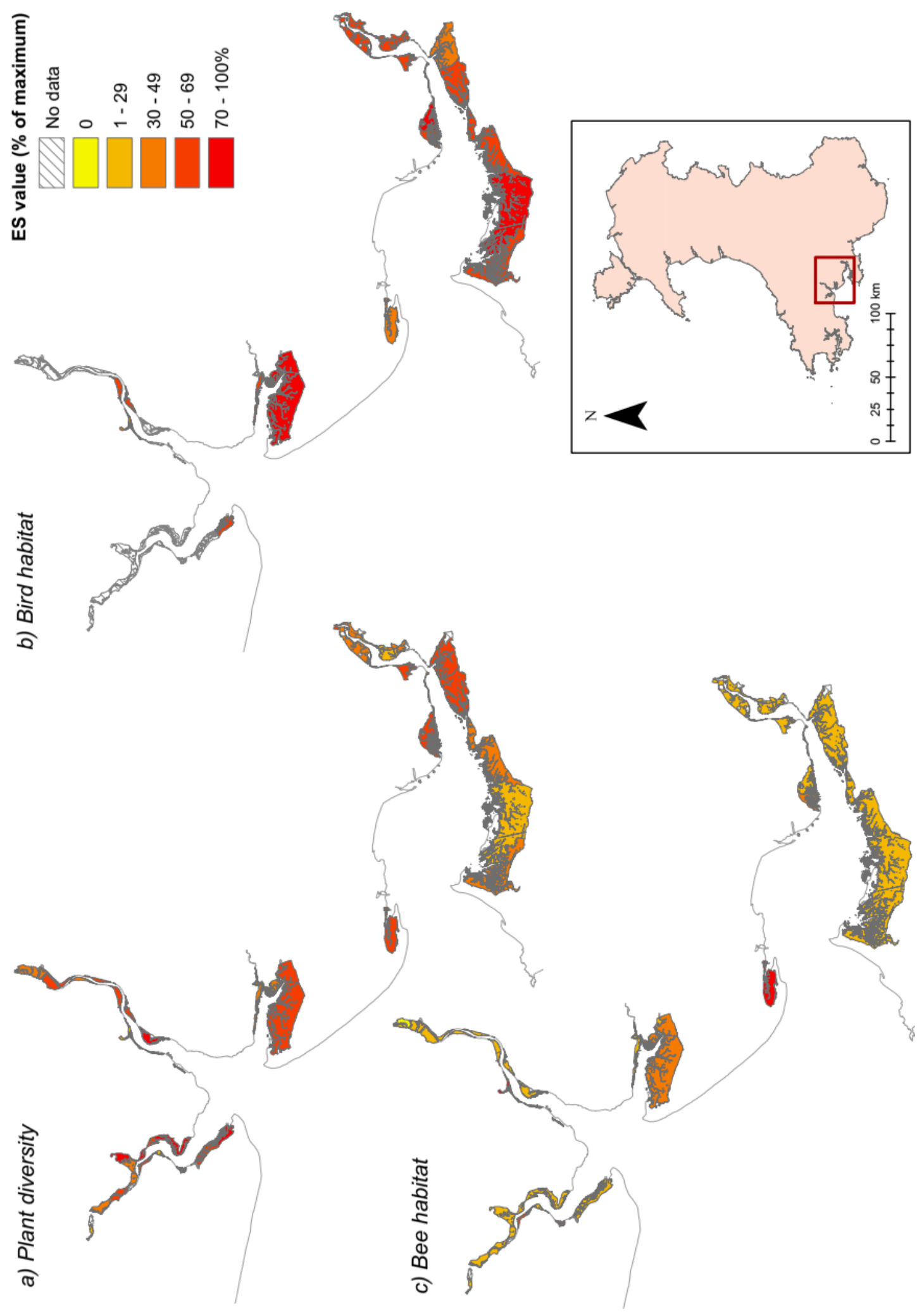

Fig. D1. Heat maps of individual ES provision. 

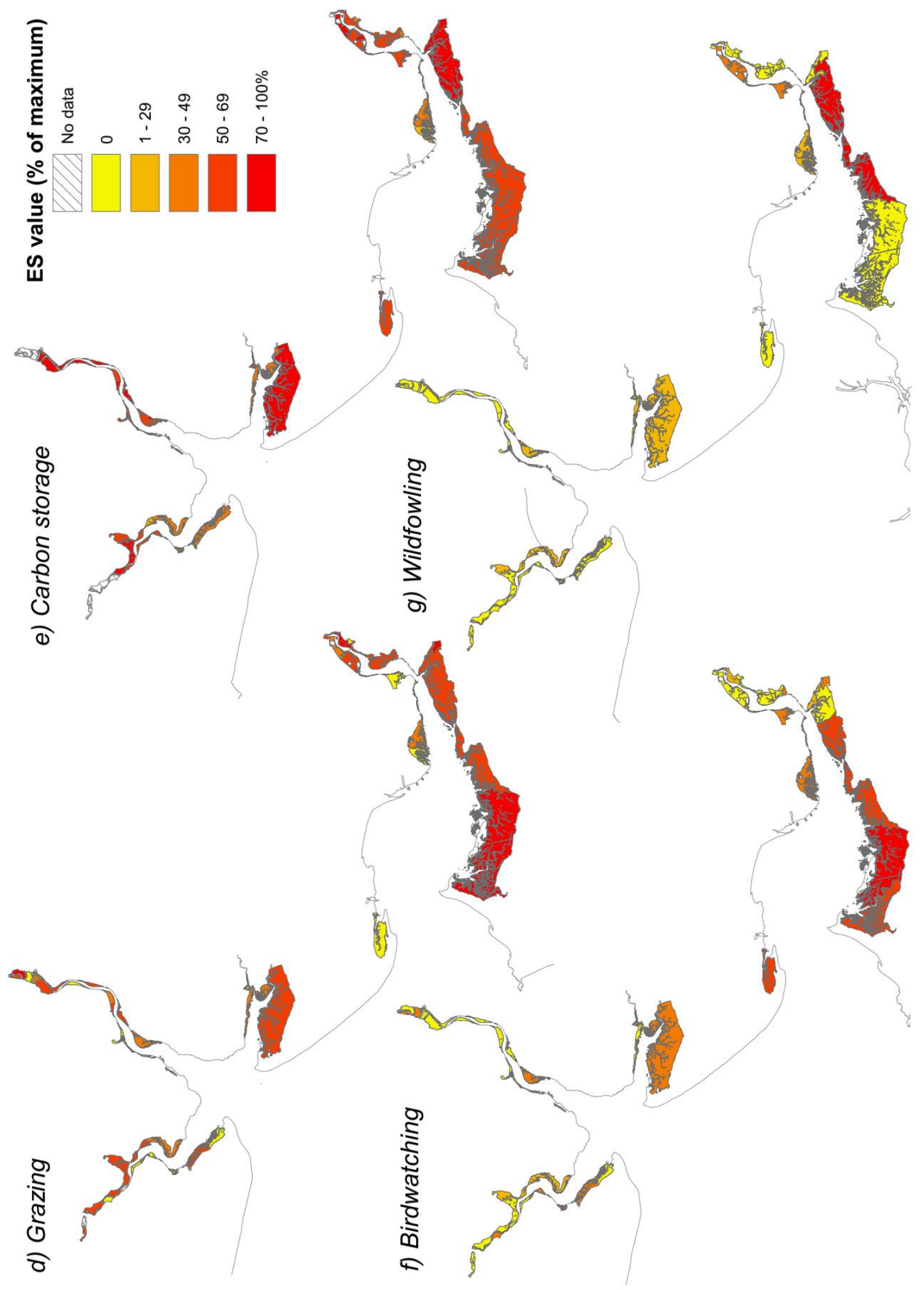

Fig. D1 cont. 


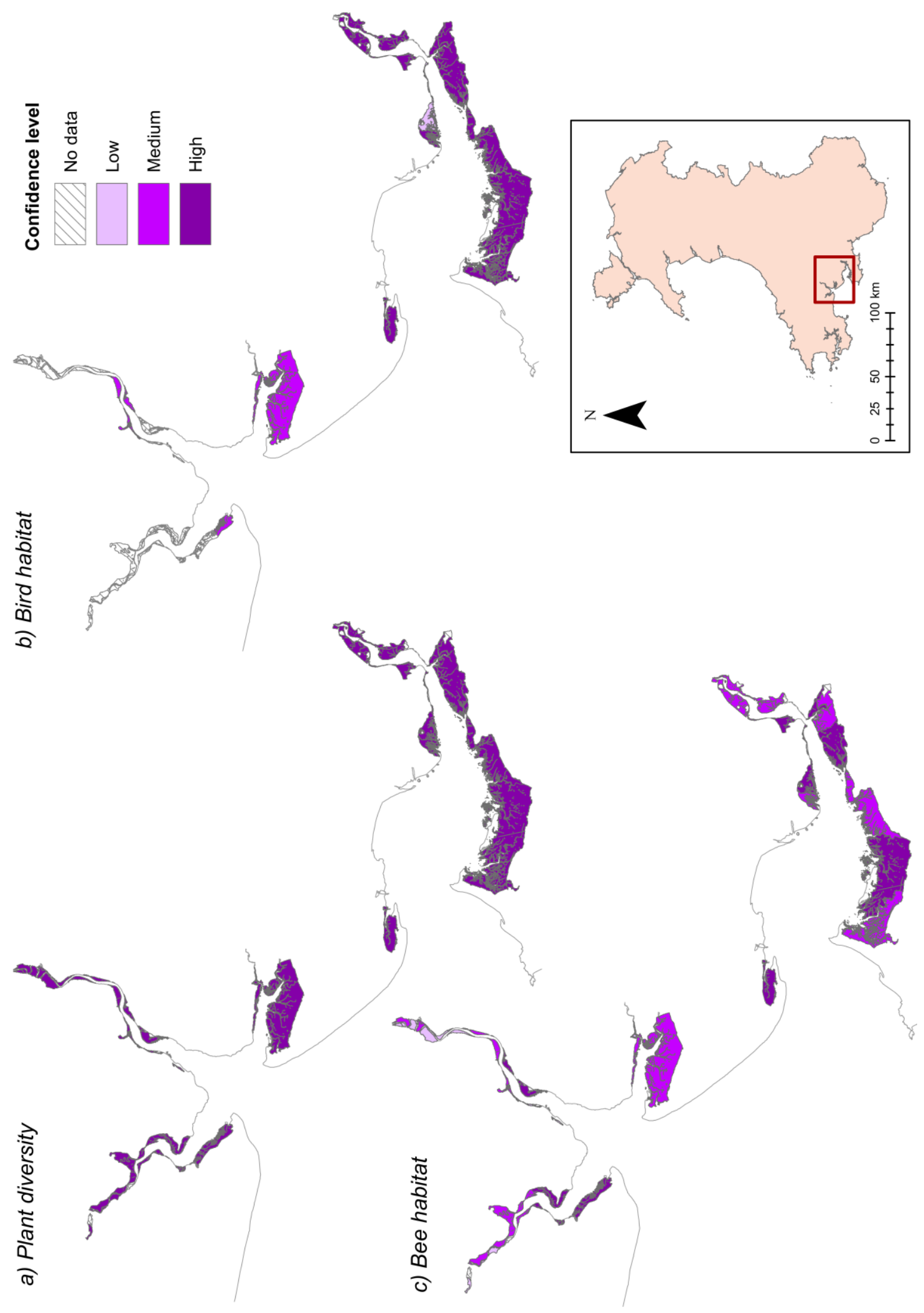

Fig. D2. Confidence in each ES map layer. 

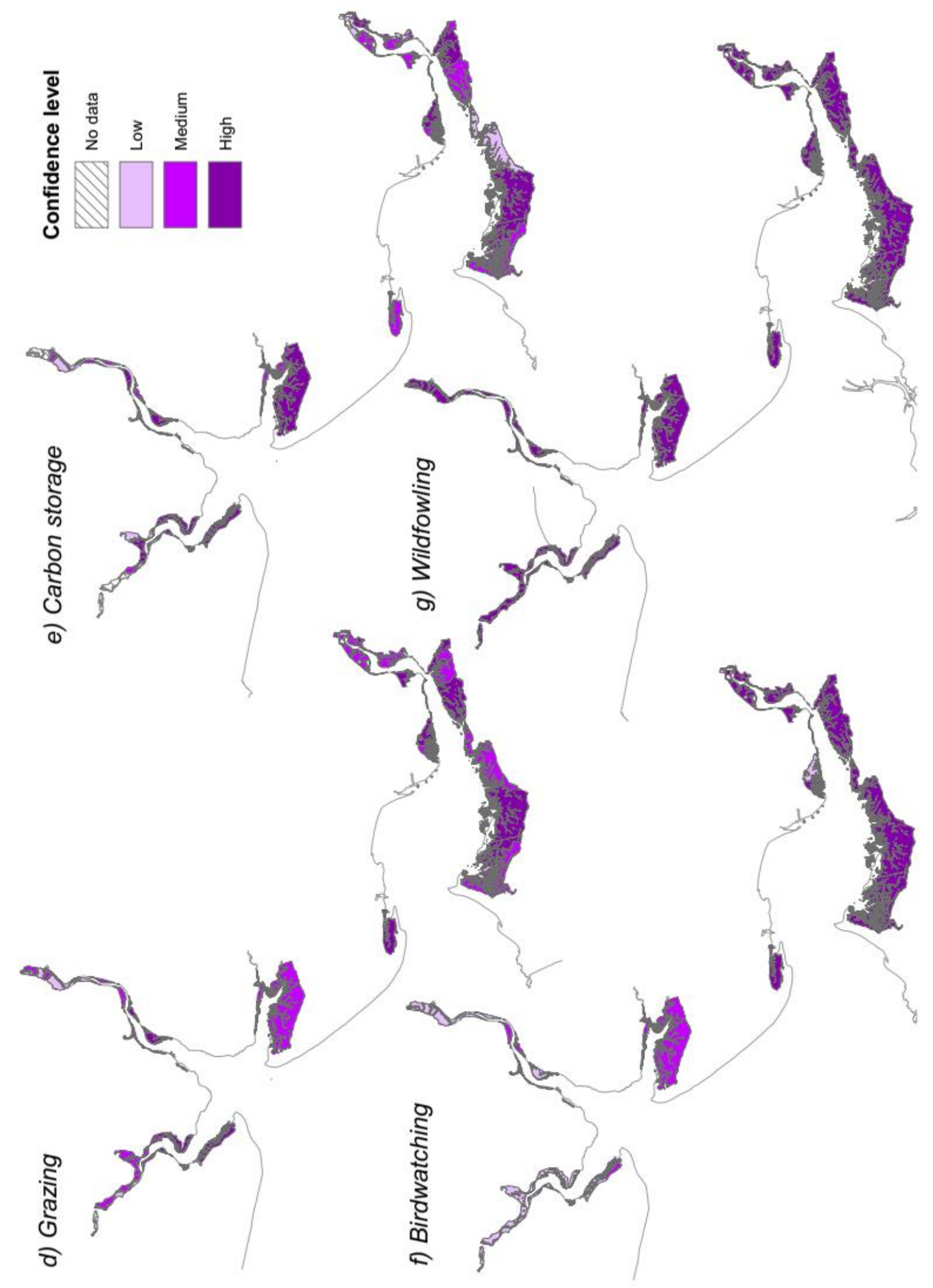

Fig. D2 cont. Confidence in each ES map layer. 


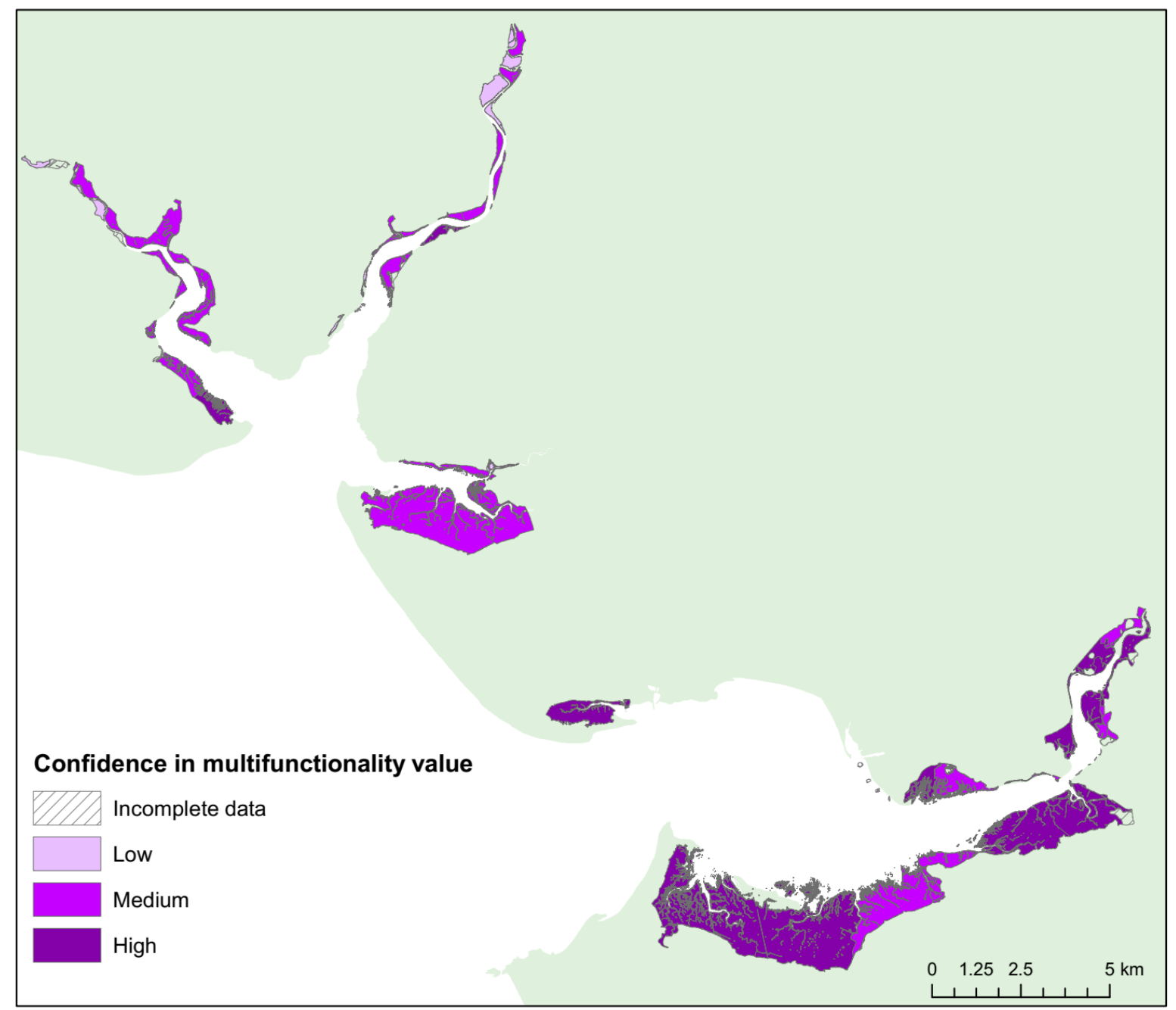

Fig. D3. Confidence levels for ES multifunctionality values and ES bundles. The confidence level was calculated by averaging across the confidence levels for each of the seven ES, where $0=$ assumed based on no WeBS survey (see section 2.3.2), 1=low, 2 =medium, 3=high confidence. The mean value was then rounded to the nearest whole number, to be assigned a confidence level of low (1), medium (2), or high (3). 
Appendix E: Owner/occupier interviews of saltmarsh management

\section{Contents}

Page

Questionnaire.

202

Table E1. Summarised responses to grazing questionnaire.

204 


\section{Questionnaire}

\section{Grazing Questionnaire}

This survey is being carried out as part of a research project on Welsh Saltmarshes (RESILCOAST, http://www.nrn-lcee.ac.uk/resilcoast/research.php.en). The project is investigating the role salt marshes play in supporting coastal communities, and how they are managed and used. One of the aspects of this is understanding how salt marshes are used for agriculture in Wales.

We would like to talk to you about your use of the marsh, through this short questionnaire. It should take approximately 15 minutes of your time.

The questionnaire is being undertaken in accordance with the Research Ethics Guidelines of Cardiff University, and has been approved by the Research Ethics Committee of the School of Earth and Ocean Sciences. Your participation in this questionnaire is entirely voluntary, and you can stop your participation at any time. All data you provide, including any personal data, will be treated with confidentiality, and will only be used for the purpose of this research study. The data will never be sold on and will not be individually attributable to you. The information you provide will be stored securely and used for research purposes only.

If you have any questions or concerns about this research, you can contact the researchers using the following e-mail address: mckinleye1@cardiff.ac.uk

\section{Please confirm you accept this and are willing to proceed with the survey by checking the box below.}

I confirm, by checking this box, that I am over 18 and I allow you to use, anonymously, the information I provide in this questionnaire.

Location of the farm:

Date of interview:

1. How long have you or your family been farming this land?

2. Do you currently farm saltmarsh land in any way?

Yes

No

3. If yes, what type of farming activity? (what type(s) of livestock/ species/ age of animals farmed)

a. Livestock type:

b. Breed:

c. Age of animals:

4. How many animals do you stock on the saltmarsh?

5. What is the approximate size of the saltmarsh area that you farm?

6. Is the area you farm one area or is it split across multiple marshes? 
7. What months of year do you graze the marsh? Please check all that apply.

January $\quad \square \quad$ February $\quad \square \quad$ March $\quad \square \quad$ April

May $\quad \square \quad$ June $\quad \square \quad$ July $\quad \square \quad$ August

September $\quad \square \quad$ October $\quad \square \quad$ November $\quad \square \quad$ December

8. Do you rotate use of the salt marsh land?

Yes $\square$ No

If yes, please explain if possible:

9. How important is the salt marsh to your general farming income?

Very Important

Important

Slightly important

Unimportant

10. Do you sell your meat as 'salt marsh' lamb/beef etc.?

Yes $\square \quad$ No

11. Is the salt marsh land that you use farmed by anyone else?

Yes $\square \quad$ No

12. Is there a right of way access on the saltmarsh that you use?

Yes $\square \quad$ No

13. Are you a tenant or the landowner of the salt marsh that you use?

Tenant

Landowner

14. If not owned by you, do the land owners require you to use the land in a certain way? Please explain:

15. Are you engaged in any kind of management scheme? E.g. Glastir, RSPB grazing regimes, etc

Yes $\square \quad$ No

If yes, please provide details if possible:

16. What other activities, if any, take place on the salt marsh that you farm?

17. What benefits do you think people can get from Welsh salt marshes? 
Table E1. Summarised responses to grazing questionnaire. Owner/occupier responses to questions related to grazing management and farm income. For grazing management, $\mathrm{C}=$ cattle, C2=young cattle $(<2$ yrs), $S=$ sheep, $P=$ ponies, ha=hectares, $y=y e a r$. Farm income indicates how important the saltmarsh grazing land is to overall income, where $\mathrm{VI}=$ very important, I=important, $\mathrm{Sl}=$ slightly important, $\mathrm{U}=\mathrm{unimportant}$. Salt marsh indicates whether the meat is marketed with a 'salt marsh' designation.

\begin{tabular}{|c|c|c|c|c|c|c|c|c|c|c|c|c|c|c|c|}
\hline \multirow[b]{2}{*}{ 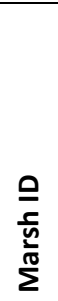 } & \multicolumn{4}{|c|}{ No. grazing animals } & \multicolumn{4}{|c|}{$\begin{array}{c}\text { No. months grazed } \\
y^{-1}\end{array}$} & \multicolumn{5}{|c|}{ Grazing intensity } & \multirow[b]{2}{*}{ 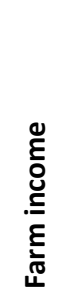 } & \multirow[b]{2}{*}{ 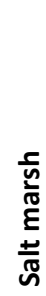 } \\
\hline & u & $\mathcal{N}$ & $n$ & a & u & $\mathcal{N}$ & $n$ & a & 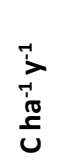 & 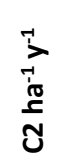 & 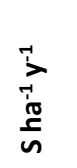 & 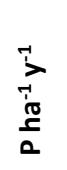 & 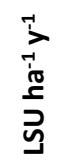 & & \\
\hline F1 & 0 & 0 & 0 & 0 & 0 & 0 & 0 & 0 & 0.00 & 0.00 & 0.00 & 0.00 & 0 & $U$ & \\
\hline A & 0 & 0 & 603 & 29 & 0 & 0 & 12 & 12 & 0.00 & 0.00 & 2.22 & 0.11 & 0.3 & 1 & yes \\
\hline B & 0 & 0 & 1270 & 61 & 0 & 0 & 12 & 12 & 0.00 & 0.00 & 2.22 & 0.11 & 0.3 & 1 & yes \\
\hline C & 0 & 0 & 627 & 30 & 0 & 0 & 12 & 12 & 0.00 & 0.00 & 2.22 & 0.11 & 0.3 & I & yes \\
\hline D1 & 0 & 0 & 1320 & 0 & 0 & 0 & 9 & 0 & 0.00 & 0.00 & 4.50 & 0.00 & 0.54 & $\mathrm{VI}$ & yes \\
\hline D2 & 0 & 0 & 480 & 0 & 0 & 0 & 9 & 0 & 0.00 & 0.00 & 4.50 & 0.00 & 0.54 & $\mathrm{VI}$ & yes \\
\hline D3 & 25 & 0 & 0 & 0 & 5 & 0 & 0 & 0 & 0.80 & 0.00 & 0.00 & 0.00 & 0.8 & $\mathrm{VI}$ & no \\
\hline G1 & $?$ & 0 & $?$ & 0 & 12 & 0 & 12 & 0 & & 0.00 & & 0.00 & (2)? & 1 & yes \\
\hline G2 & 0 & 0 & 0 & 0 & 0 & 0 & 0 & 0 & 0.00 & 0.00 & 0.00 & 0.00 & 0 & $U$ & \\
\hline J1 & 0 & 35 & 0 & 0 & 0 & 7 & 0 & 0 & 0.00 & & 0.00 & 0.00 & 0.2 & $\mathrm{VI}$ & no \\
\hline $\mathbf{L}$ & 150 & 50 & 0 & 0 & 7 & 7 & 0 & 0 & 0.16 & 0.05 & 0.00 & 0.00 & 0.2 & $\mathrm{VI}$ & no \\
\hline M3 & 10 & 0 & 0 & 0 & 6 & 0 & 0 & 0 & 0.10 & 0.00 & 0.00 & 0.00 & 0.1 & $\mathrm{SI}$ & no \\
\hline N1 & 3 & 0 & 0 & 0 & 12 & 0 & 0 & 0 & 0.30 & 0.00 & 0.00 & 0.00 & 0.3 & $\mathrm{VI}$ & no \\
\hline N2 & 15 & 0 & 0 & 0 & 12 & 0 & 0 & 0 & 0.30 & 0.00 & 0.00 & 0.00 & 0.3 & $\mathrm{VI}$ & no \\
\hline 01 & 18 & 0 & 18 & 0 & 3 & 0 & 3 & 0 & 0.28 & 0.00 & 0.28 & 0.00 & 0.3 & 1 & no \\
\hline 02 & 0 & $?$ & ? & 0 & 0 & 5 & 5 & 0 & 0.00 & & & 0.00 & 0.6 & $\mathrm{SI}$ & no \\
\hline R1 & 80 & 0 & 0 & 0 & 3 & 0 & 0 & 0 & 0.57 & 0.00 & 0.00 & 0.00 & 0.6 & $I$ & no \\
\hline R2 & 65 & 0 & 50 & 0 & 6 & 0 & 6 & 0 & 0.90 & 0.00 & 0.69 & 0.00 & 1 & $\mathrm{VI}$ & no \\
\hline U1 & 60 & 60 & 150 & 0 & 7 & 7 & 1.5 & 0 & 0.31 & 0.31 & 0.16 & 0.00 & 0.5 & $\mathrm{VI}$ & no \\
\hline
\end{tabular}


Appendix F: Expert opinion birdwatching survey

\section{Contents}

Expert opinion survey.

Page

Table F1. Summarised responses to expert opinion survey.

206

208 


\section{Expert opinion survey.}

\section{Expert opinion survey - winter birdwatching on Welsh salt marshes}

This survey is being conducted as part of a study by Swansea University into the Ecosystem Services (the ways nature benefits people) provided by the salt marshes of South Wales. The end result will be the production of Ecosystem Service maps for the Carmarthen Bay and Estuaries Special Area of Conservation (SAC), to aid NRW in their management of the site. In order to predict what marshes are best for birdwatching, we need to understand the factors that will influence a visit, and the bird sightings that are most desired.

Please complete the two sections below. If you have any further thoughts or insights, we would be very happy to hear them. Please return the form to DavidsonKE@hotmail.co.uk.

1. We would like to understand what features of a salt marsh are most influential in encouraging visits from birdwatchers. Please rank the following features from $1-8$ in terms of how likely they are to influence a visit to a particular salt marsh.

Rank from 1 (most important) - 8 (least important)

Proximity to home/work

Car parking nearby

Easy access onto site

The presence of a bird hide

Large numbers of birds present

High diversity of birds present

The presence of particular species. Please state:

Other features. Please state: 
2. We would like to know what bird sightings are the most preferred for a birdwatcher - i.e. those species that would most encourage you to visit a salt marsh for the purpose of birdwatching. The following species can all be seen during winter in the

Carmarthen Bay and Estuaries SAC. Please give each species a rating of $1-3$ in terms of how desirable a sighting is.

\begin{tabular}{|c|c|c|c|}
\hline & \multicolumn{2}{|c|}{ Most highly preferred } & Less highly preferred \\
\hline & 1 & 2 & 3 \\
\hline Bar-tailed Godwit & $\square$ & $\square$ & $\square$ \\
\hline Black-tailed Godwit & $\square$ & $\square$ & $\square$ \\
\hline Brent Goose & $\square$ & $\square$ & $\square$ \\
\hline Common Gull & $\square$ & $\square$ & $\square$ \\
\hline Common Scoter & $凶$ & $\square$ & $\square$ \\
\hline Curlew & $\square$ & $\square$ & $\square$ \\
\hline Dunlin & $\square$ & $\square$ & $\square$ \\
\hline Eider & $\square$ & $\square$ & $\square$ \\
\hline Golden Plover & $\square$ & $\square$ & $\square$ \\
\hline Goldeneye & $\square$ & $\square$ & $\square$ \\
\hline Great White Egret & $\square$ & $\square$ & $\square$ \\
\hline Green Sandpiper & $\square$ & $\square$ & $\square$ \\
\hline Greenshank & $\square$ & $\square$ & $\square$ \\
\hline Grey Plover & $\square$ & $\square$ & $\square$ \\
\hline Knot & $\square$ & $\square$ & $\square$ \\
\hline Lapwing & $\square$ & $\square$ & $\square$ \\
\hline Marsh Harrier & $\square$ & $\square$ & $\square$ \\
\hline Mediterranean Gull & $\square$ & $\square$ & $\square$ \\
\hline Merlin & $\square$ & $\square$ & $\square$ \\
\hline Pintail & $\square$ & $\square$ & $\square$ \\
\hline Pochard & $\square$ & $\square$ & $\square$ \\
\hline Red-breasted Merganser & $\square$ & $\square$ & $\square$ \\
\hline Red-throated Diver & $\square$ & $\square$ & $\square$ \\
\hline Redshank & $\square$ & $\square$ & $\square$ \\
\hline Ruff & $\square$ & $\square$ & $\square$ \\
\hline Sanderling & $\square$ & $\square$ & $\square$ \\
\hline Shoveler & $\square$ & $\square$ & $\square$ \\
\hline Slavonian Grebe & $\square$ & $\square$ & $\square$ \\
\hline Snipe & $\square$ & $\square$ & $\square$ \\
\hline Snow Goose & $\square$ & $\square$ & $\square$ \\
\hline Spotted Redshank & $\square$ & $\square$ & $\square$ \\
\hline Turnstone & $\square$ & $\square$ & $\square$ \\
\hline Whooper Swan & $\square$ & $\square$ & $\square$ \\
\hline Wigeon & $\square$ & $\square$ & $\square$ \\
\hline
\end{tabular}


Table F2. Summarised responses to expert opinion survey. Responders were as follows. A: Alistair Wilson, Natural Resources Wales, B: Ed O’Connor, Wildfowl \& Wetland Trust; C: Rachel Taylor, British Trust for Ornithology; D: Tracey Dunford, Natural Resources Wales; E: Frank Sengpiel, Cardiff University; F: Lizzie Wilberforce, Wildlife Trust of South and West Wales; G: Vaughn Matthews, Wildlife Trust of South and West Wales; H: Rich Brown, Wildlife Trust of South and West Wales.

\begin{tabular}{|c|c|c|c|c|c|c|c|c|c|c|c|c|}
\hline Feature & A & B & C & D & $E$ & $F$ & $\mathbf{G}$ & $\mathbf{H}$ & $\begin{array}{l}\text { Mea } \\
n\end{array}$ & $\begin{array}{l}\mathrm{Ma} \\
\mathrm{x}\end{array}$ & $\begin{array}{l}\mathrm{Mi} \\
\mathrm{n}\end{array}$ & $\begin{array}{l}\text { Media } \\
\mathrm{n}\end{array}$ \\
\hline Diversity & 2 & 1 & 1 & 5 & 1 & 2 & 1 & 2 & 1.9 & 1 & 5 & 1.5 \\
\hline Numbers & 1 & 2 & 5 & 4 & 4 & 3 & 2 & 3 & 3.0 & 1 & 5 & 3 \\
\hline Parking & 3 & 6 & 3 & 1 & 3 & 4 & 7 & 5 & 4.0 & 1 & 7 & 3.5 \\
\hline Access & 4 & 3 & 4 & 2 & 5 & 5 & 6 & 5 & 4.3 & 2 & 6 & 4.5 \\
\hline Hide & 5 & 8 & 2 & 3 & 2 & 6 & 5 & 4 & 4.4 & 2 & 8 & 4.5 \\
\hline $\begin{array}{l}\text { Proximit } \\
\text { y }\end{array}$ & 6 & 4 & 6 & 8 & 6 & 1 & 4 & 5 & 5.0 & 1 & 8 & 5.5 \\
\hline Species & 7 & 7 & 8 & 6 & 7 & 7 & 3 & 5 & 6.3 & 3 & 8 & 7 \\
\hline Other & 8 & 5 & 7 & 7 & 8 & 8 & 8 & 1 & 6.5 & 1 & 8 & 7.5 \\
\hline $\begin{array}{l}\text { Preferre } \\
\text { d species }\end{array}$ & & $\begin{array}{l}\text { Birds } \\
\text { of } \\
\text { prey }\end{array}$ & & $\begin{array}{l}\text { Bitter } \\
\mathrm{n}\end{array}$ & $\begin{array}{l}\text { Bitter } \\
\mathrm{n}\end{array}$ & $\begin{array}{l}\text { Rare } \\
\text { specie } \\
\mathrm{s}\end{array}$ & $\begin{array}{l}\text { Marsh } \\
\text { harrie } \\
r\end{array}$ & & & & & \\
\hline $\begin{array}{l}\text { Other } \\
\text { features }\end{array}$ & & 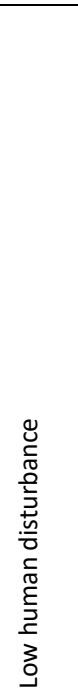 & 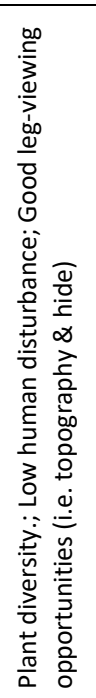 & 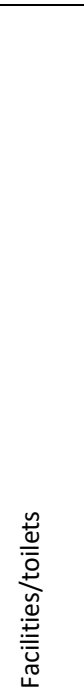 & 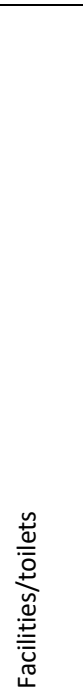 & & & 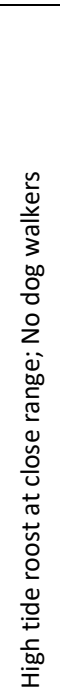 & & & & \\
\hline
\end{tabular}




\section{References}

Abdalla, M., Hastings, A., Chadwick, D.R., Jones, D.L., Evans, C.D., Jones, M.B., ... Smith, P. (2018). Critical review of the impacts of grazing intensity on soil organic carbon storage and other soil quality indicators in extensively managed grasslands. Agriculture, Ecosystems \& Environment 253:62-81. doi: https://doi.org/10.1016/j.agee.2017.10.023.

Abdi, H. and Williams, L.J. (2010). Tukey's Honestly Significant Difference (HSD) Test. In: Salkind, N. (ed.). Encyclopedia of Research Design. Thousand Oaks, CA, USA: Sage.

Adam, P. (1981). The vegetation of British saltmarshes. New Phytologist 88:143-196. doi: https://doi.org/10.1111/j.1469-8137.1981.tb04577.x.

Adam, P. (1990). Saltmarsh Ecology. Cambridge, UK: Cambridge University Press.

Adnitt, C., Brew, D., Cottle, R., Hardwick, M., John, S., Leggett, D., ... Staniland, R. (2007).

Saltmarsh Management Manual. R\&D Technical Report SC030220. Bristol, UK: Environment Agency.

Agassiz, D.J.L. (2000). Lepidoptera of British saltmarshes. In: Sherwood, B. R., Gardiner, B. G. and Harris, T. (eds.). British Saltmarshes. Ceredigion, UK: Forrest Text for The Linnean Society of London, pp. 303-308.

Alber, M., Swenson, E.M., Adamowicz, S.C. and Mendelssohn, I.A. (2008). Salt Marsh Dieback: An overview of recent events in the US. Estuarine, Coastal and Shelf Science 80(1):1-11. doi: https://doi.org/10.1016/J.ECSS.2008.08.009.

Alexander, S.K. (1979). Diet of the periwinkle Littorina irrorata in a Louisiana salt marsh. Gulf Research Reports 6(3). doi: https://doi.org/10.18785/grr.0603.11.

Alkemade, R., Reid, R.S., van den Berg, M., de Leeuw, J. and Jeuken, M. (2013). Assessing the impacts of livestock production on biodiversity in rangeland ecosystems. Proceedings of the National Academy of Sciences 110(52):20900-20905. doi: https://doi.org/10.1073/pnas.1011013108.

Allan, E., Manning, P., Alt, F., Binkenstein, J., Blaser, S., Blüthgen, N., ... Fischer, M. (2015). Land use intensification alters ecosystem multifunctionality via loss of biodiversity and changes to functional composition. Ecology Letters:834-843. doi: https://doi.org/10.1111/ele.12469.

Alldred, M. and Baines, S.B. (2016). Effects of wetland plants on denitrification rates: a metaanalysis. Ecological Applications 26(3):676-685. doi: https://doi.org/10.1890/14-1525.1.

Allen, J.R.L. (2000). Morphodynamics of Holocene salt marshes: A review sketch from the Atlantic and Southern North sea coasts of Europe. Quaternary Science Reviews 19:11551231. doi: https://doi.org/10.1016/S0277-3791(00)00157-8.

Allen, K.E. (2015). Trade-offs in nature tourism: contrasting parcel-level decisions with landscape conservation planning. Ecology and Society 20(1):21.

Altieri, A.H., Silliman, B.R. and Bertness, M.D. (2007). Hierarchical organization via a facilitation cascade in intertidal cordgrass Bed communities. The American Naturalist 169(2):195-206. doi: https://doi.org/10.1086/510603.

Anderson, M.J. (2001). A new method for non-parametric multivariate analysis of variance. Austral Ecology 26:32-46.

Anderson, M.J. (2006). Distance-based tests for homogeneity of multivariate dispersions. 
Biometrics 62:245-253.

Andresen, H., Bakker, J.P., Brongers, M., Heydemann, B. and Irmler, U. (1990). Long-term changes of salt marsh communities by cattle grazing. Vegetatio 89:137-148. doi: https://doi.org/10.1007/BF00032166.

Andriuzzi, W.S. and Wall, D.H. (2017). Responses of belowground communities to large aboveground herbivores: meta-analysis reveals biome-dependent patterns and critical research gaps. Global Change Biology. doi: https://doi.org/10.1111/gcb.13675.

Angelini, C., Griffin, J.N., van de Koppel, J., Derksen-Hooijberg, M., Smolders, A.J.P., Lamers, L., ... Silliman, B.R. (2016). A keystone mutualism underpins resilience of a coastal system to drought. Nature Communications 7:1-8. doi: https://doi.org/10.1038/ncomms12473.

Angelini, C., van der Heide, T., Griffin, J.N., Morton, J.P., Derksen-Hooijberg, M., Lamers, L.P.M., ... Silliman, B.R. (2015). Foundation species' overlap enhances biodiversity and multifunctionality from the patch to landscape scale in southeastern United States salt marshes. Proceedings of the Royal Society B 282:20150421. doi:

https://doi.org/10.1098/rspb.2015.0421.

Angelini, C., Montfrans, S.G. Van, Hensel, M.J.S., He, Q. and Silliman, B.R. (2018). The importance of an underestimated grazer under climate change: how crab density, consumer competition, and physical stress affect salt marsh resilience. Oecologia 187:205-217.

Arkema, K.K., Guannel, G., Verutes, G., Wood, S. a., Guerry, A., Ruckelshaus, M., ... Silver, J.M. (2013). Coastal habitats shield people and property from sea-level rise and storms. Nature Climate Change 3:913-918. doi: https://doi.org/10.1038/nclimate1944.

Arkema, K.K., Verutes, G.M., Wood, S.A., Clarke-Samuels, C., Rosado, S., Canto, M., ... Guerry, A.D. (2015). Embedding ecosystem services in coastal planning leads to better outcomes for people and nature. Proceedings of the National Academy of Sciences 112(24):7390-7395. doi: https://doi.org/10.1073/pnas.1406483112.

Armonies, W. (1986). Free-living plathelminthes in sheep-grazed and ungrazed supralittoral salt marshes of the North Sea: Abundance, biomass, and their significance in food chains. Netherlands Journal of Sea Research 20(4):385-395. doi: https://doi.org/10.1016/00777579(86)90005-0.

Atia, A., Rabhi, M., Debez, A., Abdelly, C., Gouia, H., Haouari, C.C. and Smaoui, A. (2014). Ecophysiological aspects in 105 plants species of saline and arid environments in Tunisia. Journal of Arid Land 6:762-770. doi: https://doi.org/10.1007/s40333-014-0028-2.

Bai, J., Zhang, G., Zhao, Q., Lu, Q., Jia, J., Cui, B. and Liu, X. (2016). Depth-distribution patterns and control of soil organic carbon in coastal salt marshes with different plant covers.

Scientific Reports 6:34835. doi: https://doi.org/10.1038/srep34835.

Baker, H.K., Nelson, J.A. and Leslie, H.M. (2016). Quantifying striped bass (Morone saxatilis) dependence on saltmarsh-derived productivity using stable isotope analysis. Estuaries and Coasts 39:1537-1542. doi: https://doi.org/10.1007/s12237-016-0092-2.

Bakker, J.P., Bos, D. and De Vries, Y. (2003). To graze or not to graze: that is the question. In: Essink, K., van Leeuwe, M., Kellermann, A. and Wolff, W. (eds.). Proceedings of the 10th International Scientific Wadden Sea Symposium. Netherlands: Ministry of Agriculture, Nature management and Fisheries, The Hague, and Department of Marine Biology, University of Groningen, pp. 67-88.

Bakker, J.P., Nielsen, K.J., Alberti, J., Chan, F., Hacker, S.D., Iribarne, O.O., ... Silliman, B.R. (2015). Bottom-up and top-down interactions in coastal interface systems. In: Hanley, T. C. 
and La Pierre, K. J. (eds.). Trophic Ecology: Bottom-Up and Top-Down Interactions across Aquatic and Terrestrial Systems. Cambridge: Cambridge University Press, pp. 157-200.

Baldock, K.C.R., Goddard, M.A., Hicks, D.M., Kunin, E., Mitschunas, N., Osgathorpe, L.M., ... Memmott, J. (2015). Where is the UK's pollinator biodiversity? The importance of urban areas for flower-visiting insects. Proceedings of the Royal Society B-Biological Sciences 282:20142849. doi: https://doi.org/10.1098.

Balvanera, P., Siddique, I., Dee, L., Paquette, A., Isbell, F., Gonzalez, A., ... Griffin, J.N. (2014). Linking biodiversity and ecosystem services: Current uncertainties and the necessary next steps. BioScience 64(1):49-57. doi: https://doi.org/10.1093/biosci/bit003.

Barbier, E.B. (2015). Valuing the storm protection service of estuarine and coastal ecosystems. Ecosystem Services 11:32-38. doi: https://doi.org/10.1016/j.ecoser.2014.06.010.

Barbier, E.B., Hacker, S.D., Kennedy, C., Koch, E.W., Stier, A.C. and Silliman, B.R. (2011). The value of estuarine and coastal ecosystem services. Ecological Monographs 81(2):169-193. doi: https://doi.org/10.1890/0012-9615-81.2.169.

Barr, K. and Bell, M. (2016). Neolithic and Bronze Age ungulate footprint-tracks of the Severn Estuary: Species, age, identification and the interpretation of husbandry practices. Environmental Archaeology. doi: https://doi.org/10.1080/14614103.2016.1222691.

Baselga, A. (2010). Partitioning the turnover and nestedness components of beta diversity. Global Ecology and Biogeography 19(1):134-143. doi: https://doi.org/10.1111/j.14668238.2009.00490.x.

Baselga, A. and Orme, C.D.L. (2012). betapart: An R package for the study of beta diversity. Methods in Ecology and Evolution 3(5):808-812. doi: https://doi.org/10.1111/j.2041210X.2012.00224.x.

Bates, D., Maechler, M., Bolker, B. and Waler, S. (2015). Fitting linear mixed-effects models using Ime4. Journal of Statistical Software 67. doi: https://doi.org/10.18637/jss.v067.i01.

Baugh, T.M., Valade, J.A. and Zoodsma, B.J. (1989). Manatee use of Spartina alterniflora in Cumberland Sound. Marine Mammal Science 5(1):88-90.

Beaumont, N.J., Mongruel, R. and Hooper, T. (2017). Practical application of the Ecosystem Service Approach (ESA): lessons learned and recommendations for the future. International Journal of Biodiversity Science, Ecosystem Services \& Management 13(3):68-78. doi: https://doi.org/10.1080/21513732.2018.1425222.

Bell, F.W. (1997). The economic valuation of saltwater marsh supporting marine recreational fishing in the southeastern United States. Ecological Economics 21(3):243-254. doi: https://doi.org/10.1016/S0921-8009(96)00105-X.

Bell, L.W., Kirkegaard, J.A., Swan, A., Hunt, J.R., Huth, N.I. and Fettell, N.A. (2011). Impacts of soil damage by grazing livestock on crop productivity. Soil and Tillage Research 113(1):19-29. doi: https://doi.org/10.1016/j.still.2011.02.003.

Belsky, J.A. and Gelbard, J.L. (2000). Livestock Grazing and Weed Invasions in the Arid West. Bend, OR.

Benjamini, Y. and Hochberg, Y. (1995). Controlling the False Discovery Rate: A practical and powerful approach to multiple testing. Journal of the Royal Statistical Society. Series $B$ (Methodological) 57(1):289-300.

Bennett, E.M. (2016). Research frontiers in ecosystem service science. Ecosystems:1-7. doi: 
https://doi.org/10.1007/s10021-016-0049-0.

Bennett, E.M., Peterson, G.D. and Gordon, L.J. (2009). Understanding relationships among multiple ecosystem services. Ecology Letters 12:1394-1404. doi: https://doi.org/10.1111/j.1461-0248.2009.01387.x.

Bennett, J.A., Gensler, G.C. and Cahill, J.F. (2014). Small-scale bee patch use is affected equally by flower availability and local habitat configuration. Basic and Applied Ecology 15(3):260-268. doi: https://doi.org/10.1016/j.baae.2014.03.004.

Bergelson, J., Newman, J.A. and Floresroux, E.M. (1993). Rates of weed spread in spatially heterogeneous environments. Ecology 74(4):999-1011. doi:

https://doi.org/10.2307/1940470.

Bertness, M.D. (1985). Fiddler crab regulation of Spartina alterniflora production on a New England salt marsh. Ecology 66(3):1042-1055. doi: https://doi.org/10.2307/1940564.

Blum, L.K. (1993). Spartina alterniflora root dynamics in a Virginia marsh. Marine Ecology Progress Series1 102:169-178.

Boerema, A., Rebelo, A.J., Bodi, M.B., Esler, K.J. and Meire, P. (2016). Are ecosystem services adequately quantified? Journal of Applied Ecology. doi: https://doi.org/10.1111/13652664.12696.

Böhme, B., Främbs, H., Hielen, B. and Kinder, M. (1999). Salt marsh project 'Wurster Kuste': Regeneration of coastal grasslands and salt marshes. Senckenbergiana Maritima 29(Suppl.):37-42.

Boorman, L.A. (1967). Limonium vulgare Mill. and L. humile Mill. Journal of Ecology 55(1):221-232.

Bos, D., Loonen, M.J.J.E., Stock, M., Hofeditz, F., van der Graaf, A.J. and Bakker, J.P. (2005). Utilisation of Wadden Sea salt marshes by geese in relation to livestock grazing. Journal for Nature Conservation 13(1):1-15. doi: https://doi.org/10.1016/j.jnc.2004.10.001.

Bouchard, V., Tessier, M., Digaire, F., Vivier, J.P., Valery, L., Gloaguen, J.C. and Lefeuvre, J.C. (2003). Sheep grazing as management tool in Western European saltmarshes. Comptes Rendus - Biologies 326(Suppl.):S148-S157.

Boyd, B.M. and Sommerfield, C.K. (2016). Marsh accretion and sediment accumulation in a managed tidal wetland complex of Delaware Bay. Ecological Engineering 92:37-46. doi: https://doi.org/10.1016/j.ecoleng.2016.03.045.

Breheny, P. and Burchett, W. (2017). Visualization of Regression Models Using visreg. The $R$ Journal 9:56-71.

Briske, D.D. and Richards, J.H. (1995). Plant responses to defoliation: A physiological, morphological and demographic evaluation. In: Bedunah, D. J. and Sosebee, R. E. (eds.). Wildland Plants: Physiological Ecology and Developmental Morphology. Denver, CO: Society for Range Management, pp. 635-710.

British Trust for Ornithology (2019). Wetland Bird Survey. Available at:

https://www.bto.org/our-science/projects/webs/about [Accessed: 21 July 2019].

Broekx, S., Liekens, I., Peelaerts, W., De Nocker, L., Landuyt, D., Staes, J., ... Cerulus, T. (2013). A web application to support the quantification and valuation of ecosystem services.

Environmental Impact Assessment Review 40:65-74. doi:

https://doi.org/10.1016/j.eiar.2013.01.003. 
Brooks, M.E., Kristensen, K., van Benthem, K.J., Magnusson, A., Berg, C.W., Nielsen, A., ... Bolker, B.M. (2017). glmmTMB balances speed and flexibility among packages for Zeroinflated Generalized Linear Mixed Modeling. The R Journal 9(2):378-400.

Brown, M.J.F.F. and Paxton, R.J. (2009). The conservation of bees : a global perspective. Apidologie 40:410-416. doi: https://doi.org/10.1051/apido/2009019.

Bruhl, J. and Wilson, K. (2007). Towards a comprehensive survey of C3 and C4 photosynthetic pathways in Cyperaceae. Aliso 23(1):99-148.

Bruins, R.J.F., Canfield, T.J., Duke, C., Kapustka, L., Nahlik, A.M. and Schäfer, R.B. (2016). Using ecological production functions to link ecological processes to ecosystem services. Integrated Environmental Assessment and Management 13(1):52-61. doi: https://doi.org/10.1002/ieam.1842.

Bruppacher, L., Pellet, J., Arlettaz, R. and Humbert, J. (2016). Simple modifications of mowing regime promote butterflies in extensively managed meadows: Evidence from field-scale experiments. Biological Conservation 196:196-202. doi:

https://doi.org/10.1016/j.biocon.2016.02.018.

Burkhard, B. and Maes, J. (eds.) (2017). Mapping Ecosystem Services. Sofia: Pensoft Publishers.

Byrnes, J.E.K., Gamfeldt, L., Isbell, F., Lefcheck, J.S., Griffin, J.N., Hector, A., ... Emmett Duffy, J. (2014). Investigating the relationship between biodiversity and ecosystem multifunctionality: challenges and solutions Freckleton, R. (ed.). Methods in Ecology and Evolution 5(2):111-124. doi: https://doi.org/10.1111/2041-210X.12143.

Cadwalladr, D.A. and Morley, J. V. (1974). Further experiments on the management of saltings pasture for Wigeon (Anas penelope L.) conservation at Bridgwater Bay National Nature Reserve, Somerset. The Journal of Applied Ecology 11(2):461-466. doi: https://doi.org/10.2307/2402200.

Cahoon, D.R. and Turner, R.E. (1989). Accretion and canal impacts in a rapidly subsiding wetland II. Feldspar marker horizon technique. Estuaries 12(4):260-268. doi: https://doi.org/10.2307/1351905.

Callaway, R.M., Kikodze, D., Chiboshvili, M. and Khetsuriani, L. (2005). Unpalatable plants protect neighbours from grazing and increase plant community diversity. Ecology 86(7):1856-1862. doi: https://doi.org/10.1890/04-0784.

Cardinale, B.J., Duffy, J.E., Gonzalez, A., Hooper, D.U., Perrings, C., Venail, P., ... Naeem, S. (2012). Biodiversity loss and its impact on humanity. Nature 486:59-67. doi: https://doi.org/10.1038/nature11148.

Cardoni, D.A., Isacch, J.P. and Iribarne, O. (2015). Avian responses to varying intensity of cattle production in Spartina densiflora saltmarshes of south-eastern South America. Emu 115(1):12-19. doi: https://doi.org/10.1071/MU13028.

Cattrijsse, A. and Hampel, H. (2006). European intertidal marshes: A review of their habitat functioning and value for aquatic organisms. Marine Ecology Progress Series 324:293-307. doi: https://doi.org/10.3354/meps324293.

Cavender-bares, J., Polasky, S., King, E. and Balvanera, P. (2015). A sustainability framework for assessing trade-offs in ecosystem services. Ecology and Society 20(1):17.

Chambers, R.M., Meyerson, L.A. and Saltonstall, K. (1999). Expansion of Phragmites australis into tidal wetlands of North America. Aquatic Botany 64(3-4):261-273. doi: 
https://doi.org/10.1016/S0304-3770(99)00055-8.

Chan, K.M.A., Balvanera, P., Benessaiah, K., Chapman, M., Díaz, S., Gómez-Baggethun, E., ... Turner, N. (2016). Opinion: Why protect nature? Rethinking values and the environment. Proceedings of the National Academy of Sciences 113(6):1462-1465. doi: https://doi.org/10.1073/pnas.1525002113.

Chan, K.M.A., Goldstein, J., Satterfield, T., Hannahs, N., Kikiloi, K., Naidoo, R., ... Woodside, U. (2011). Cultural services and non-use values. In: Kareiva, P., Tallis, H., Ricketts, T. H., Daily, G. C. and Polasky, S. (eds.). Natural Capital: Theory and Practice of Mapping Ecosystem Services. Oxford, UK: Oxford University Press, pp. 206-228.

Chan, K.M.A., Guerry, A.D., Balvanera, P., Klain, S., Satterfield, T., Basurto, X., ... Woodside, U. (2012). Where are cultural and social in ecosystem services? A framework for constructive engagement. BioScience 62(8):744-756. doi: https://doi.org/10.1525/bio.2012.62.8.7.

Chatters, C. (2004). Grazing domestic animals on British saltmarshes. British Wildlife 15(6):392-400.

Chillo, V., Ojeda, R.A., Capmourteres, V. and Anand, M. (2016). Functional diversity loss with increasing livestock grazing intensity in drylands: the mechanisms and their consequences depend on the taxa. Journal of Applied Ecology. doi: https://doi.org/10.1111/13652664.12775.

Chmura, G.L. (2009). Tidal salt marshes. In: Laffoley, D. d'A. and Grimsditch, G. (eds.). The Management of Natural Coastal Carbon Sinks. Gland, Switzerland: IUCN, pp. 5-11.

Chmura, G.L., Anisfeld, S.C., Cahoon, D.R. and Lynch, J.C. (2003). Global carbon sequestration in tidal, saline wetland soils. Global Biogeochemical Cycles 17(4):22.1-22.12. doi: https://doi.org/10.1029/2002GB001917.

Clay, G.R. and Daniel, T.C. (2000). Scenic landscape assessment: the effects of land management jurisdiction on public perception of scenic beauty. Landscape and Urban Planning 49:1-13.

Colclough, S., Fonseca, L., Astley, T., Thomas, K. and Watts, W. (2005). Fish utilisation of managed realignments. Fisheries Management and Ecology 12(6):351-360. doi: https://doi.org/10.1111/j.1365-2400.2005.00467.x.

Connell, J.H. (1978). Diversity in tropical rain forests and coral reefs. Science 199:1302-1310. doi: https://doi.org/10.1126/science.199.4335.1302.

Costa, C.S.., Iribarne, O.O. and Farina, J.M. (2009). Human impacts and threats to the conservation of South American salt marshes. In: Silliman, B. R., Grosholz, E. and Bertness, M. D. (eds.). Human Impacts on Salt Marshes: A Global Perspective. Berkeley and Los Angeles, California: University of California Press, Ltd., pp. 337-360.

Costanza, R., D’Arge, R., de Groot, R., Farber, S., Grasso, M., Hannon, B., ... van den Belt, M. (1997). The value of the world's ecosystem services and natural capital. Nature 387:253-260. doi: https://doi.org/10.1038/387253a0.

Costanza, R., Pérez-Maqueo, O., Martinez, M.L., Sutton, P., Anderson, S.J. and Mulder, K. (2008). The value of coastal wetlands for hurricane protection. AMBIO: A Journal of the Human Environment 37(4):241-248. doi: https://doi.org/10.1579/00447447(2008)37[241:TVOCWF]2.0.CO;2.

Couvillon, M.J., Schürch, R. and Ratnieks, F.L.W. (2014). Waggle dance distances as integrative indicators of seasonal foraging challenges. PLOS ONE 9(4):1-7. doi: 
https://doi.org/10.1371/journal.pone.0093495.

Coverdale, T.C., Altieri, A.H. and Bertness, M.D. (2012). Belowground herbivory increases vulnerability of New England salt marshes to die-off. Ecology 93(9):2085-94.

Craft, C.B., Seneca, E.D. and Broome, S.W. (1991). Loss on ignition and kjeldahl digestion for estimating organic carbon and total nitrogen in estuarine marsh soils: Calibration with dry combustion. Estuaries 14(2):175-179. doi: https://doi.org/10.1007/BF02689350.

Crosby, S.C., Sax, D.F., Palmer, M.E., Booth, H.S., Deegan, L.A., Bertness, M.D. and Leslie, H.M. (2016). Salt marsh persistence is threatened by predicted sea-level rise. Estuarine, Coastal and Shelf Science 181:93-99. doi: https://doi.org/10.1016/j.ecss.2016.08.018.

Cymbaluk, N.F. (1990). Comparison of forage digestion by cattle and horses. Canadian Journal of Animal Science 70(2):601-610. doi: https://doi.org/10.4141/cjas90-072.

Daily, G. (1997). Nature's Services: Societal Dependence on Natural Ecosystems. Daily, G. C. (ed.). Washington, DC: Island Press.

Daleo, P., Fanjul, E., Casariego, A.M., Silliman, B.R., Bertness, M.D. and Iribarne, O. (2007). Ecosystem engineers activate mycorrhizal mutualism in salt marshes. Ecology Letters 10(10):902-908. doi: https://doi.org/10.1111/j.1461-0248.2007.01082.x.

Daniel, T.C., Muhar, A., Arnberger, A., Aznar, O., Boyd, J.W., Chan, K.M.A., ... von der Dunk, A. (2012). Contributions of cultural services to the ecosystem services agenda. Proceedings of the National Academy of Sciences of the United States of America 109(23):8812-9. doi: https://doi.org/10.1073/pnas.1114773109.

Daskin, J.H. and Pringle, R.M. (2016). Does primary productivity modulate the indirect effects of large herbivores? A global meta-analysis. Journal of Animal Ecology 85:857-868. doi: https://doi.org/10.1111/1365-2656.12522.

Davidson, K.E., Fowler, M.S., Skov, M.W., Doerr, S.H., Beaumont, N. and Griffin, J.N. (2017). Livestock grazing alters multiple ecosystem properties and services in salt marshes: a metaanalysis. Journal of Applied Ecology 54(5). doi: https://doi.org/10.1111/1365-2664.12892.

Davidson, K.E. and Griffin, J.N. (2018). Saltmarsh Ecosystem Services in Carmarthen Bay and Estuaries SAC. NRW Evidence Report Series, Report No: 260. Bangor.

Dawson, H.J. and Ingrouille, M.J. (1995). A biometric survey of Limonium vulgare Miller and L. humile Miller in the British Isles. Watsonia 20:239-254.

DeLaune, R.D. and Reddy, K.R. (2005). Redox potential. In: Hillel, D. (ed.). Encyclopedia of Soils in the Environment. Oxford, UK: Elsevier/Academic Press, pp. 366-371.

Devane, M. and Gilpin, B. (2015). Human health risks of faecal pollution from different sources: A review of the literature.

Dijkema, K.D.S. (1990). Salt and brackish marshes around the Baltic Sea and adjacent parts of the North Sea: Their vegetation and management. Biological Conservation 51(3):191-209. doi: https://doi.org/10.1016/0006-3207(90)90151-E.

Dijkstra, J.A., Boudreau, J. and Dionne, M. (2012). Species-specific mediation of temperature and community interactions by multiple foundation species. Oikos 121(5):646-654. doi: https://doi.org/10.1111/j.1600-0706.2011.19712.x.

Dolan, P.A. (2002). Effects of Grazing by Feral Horses on Dune and Salt Marsh Vegetation at Cumberland Island, Georgia. Georgia Southern University. 
Duncan, C., Thompson, J.R. and Pettorelli, N. (2015). The quest for a mechanistic understanding of biodiversity - ecosystem services relationships. Proceedings of the Royal Society B 282:20151348.

Duursma, R. (2019). bootpredictIme4: Predict Method For Ime4 With Bootstrap. R package version 0.1 .

Eaton, M., Aebischer, N., Brown, A., Hearn, R., Lock, L., Musgrove, A., ... Gregory, R. (2015). Birds of Conservation Concern 4: the population status of birds in the UK, Channel Islands and Isle of Man. British Birds 108:708-746.

Ebrahimi, A., Milotić, T. and Hoffmann, M. (2010). A herbivore specific grazing capacity model accounting for spatio-temporal environmental variation: A tool for a more sustainable nature conservation and rangeland management. Ecological Modelling 221(6):900-910. doi: https://doi.org/10.1016/J.ECOLMODEL.2009.12.009.

Eisikowitch, D. and Woodell, S.R.J. (1975). Some aspects of pollination ecology of Armeria maritima (Mill.) Willd. in Britain. New Phytologist 74:307-322.

Ellis, E.C., Pascual, U. and Mertz, O. (2019). Ecosystem services and nature's contribution to people: negotiating diverse values and trade-offs in land systems. Current Opinion in Environmental Sustainability 38:86-94. doi: https://doi.org/10.1016/j.cosust.2019.05.001.

Elschot, K., Bakker, J., Temmerman, S., van de Koppel, J. and Bouma, T. (2015). Ecosystem engineering by large grazers enhances carbon stocks in a tidal salt marsh. Marine Ecology Progress Series 537:9-21. doi: https://doi.org/10.3354/meps11447.

Elschot, K., Bouma, T.J., Temmerman, S. and Bakker, J.P. (2013). Effects of long-term grazing on sediment deposition and salt-marsh accretion rates. Estuarine, Coastal and Shelf Science 133:109-115. doi: https://doi.org/10.1016/j.ecss.2013.08.021.

Emmett, B.A., Reynolds, B., Chamberlain, P.M., Rowe, E., Spurgeon, D., Brittain, S.A., ... Woods, C. (2010). Countryside Survey: Soils Report from 2007. CS Technical Report No. 9/07. Wallingford.

Emmett, B.E. and the GMEP team (2017). Glastir Monitoring \& Evaluation Programme. Final Report to Welsh Government - Executive Summary (Contract Reference: C147/2010/11). NERC/Centre for Ecology \& Hydrology (CEH Projects: NEC04780/NEC05371/NEC05782).

EPA (2019). Agricultural Pasture, Rangeland and Grazing.United States Environmental Protection Agency. Available at: https://www.epa.gov/agriculture/agricultural-pasturerangeland-and-grazing [Accessed: 11 September 2019].

Erwin, R.M., Allen, D.H. and Jenkins, D. (2003). Created versus natural coastal islands: Atlantic waterbird populations, habitat choices, and management implications. Estuaries 26(4):949-955. doi: https://doi.org/10.1007/BF02803353.

Evans, L., Cherrett, N. and Pemsl, D. (2011). Assessing the impact of fisheries comanagement interventions in developing countries: A meta-analysis. Journal of Environmental Management 92(8):1938-1949. doi: https://doi.org/10.1016/j.jenvman.2011.03.010.

Falk, S. and Lewington, R. (2015). Field Guide to the Bees of Great Britain and Ireland. London: Bloomsbury.

Feagin, R.A., Smith, W.K., Psuty, N.P., \{\{ D.R.Y., Luisa, M., \{\{ M., ... Koske, R.E. (2010). Barrier Islands: Coupling anthropogenic stability with ecological sustainability. Journal of Coastal Research 26(5). doi: https://doi.org/10.2112/09-1185.1. 
Le Féon, V., Schermann-Legionnet, A., Delettre, Y., Aviron, S., Billeter, R., Bugter, R., ... Burel, F. (2010). Intensification of agriculture, landscape composition and wild bee communities: A large scale study in four European countries. Agriculture, Ecosystems and Environment 137(1-2):143-150. doi: https://doi.org/10.1016/j.agee.2010.01.015.

Finch, H.J.S., Samuel, A.M., Lane, G.P.F., Finch, H.J.S., Samuel, A.M. and Lane, G.P.F. (2014). Grazing management. Lockhart \& Wiseman's Crop Husbandry Including Grassland. Woodhead Publishing, pp. 499-512.

Ford, H. (2012). Biodiversity, Ecosystem Function and Ecosystem Service Provision in Saltmarsh and Sand Dune. Bangor University.

Ford, H., Garbutt, A., Jones, L. and Jones, D.L. (2012). Methane, carbon dioxide and nitrous oxide fluxes from a temperate salt marsh: Grazing management does not alter Global Warming Potential. Estuarine, Coastal and Shelf Science 113:182-191. doi: https://doi.org/10.1016/j.ecss.2012.08.002.

Ford, H., Garbutt, A., Jones, D.L. and Jones, L. (2012b). Impacts of grazing abandonment on ecosystem service provision: Coastal grassland as a model system. Agriculture, Ecosystems \& Environment 162:108-115. doi: https://doi.org/10.1016/j.agee.2012.09.003.

Ford, H., Garbutt, A., Jones, L. and Jones, D.L. (2013). Grazing management in saltmarsh ecosystems drives invertebrate diversity, abundance and functional group structure. Insect Conservation and Diversity 6(2):189-200. doi: https://doi.org/10.1111/j.17524598.2012.00202.x.

Ford, H., Garbutt, A., Ladd, C., Malarkey, J. and Skov, M.W. (2016). Erosion stabilisation linked to plant diversity and environmental context in coastal grasslands. Journal of Vegetation Science:1-10. doi: https://doi.org/10.1111/jvs.12367.

France-Presse, A. (2015). Bees are worth billions to farmers across the globe, study suggests. The Guardian.

Free, J.B. (1963). The flower constancy of honeybees. The Journal of Animal Ecology 32(1):119-131. doi: https://doi.org/10.2307/2521.

Friese, J., Temming, A. and Dänhardt, A. (2018). Grazing management affects fish diets in a Wadden Sea salt marsh. Estuarine, Coastal and Shelf Science 212:341-352.

Furbish, C.E. and Albano, M. (1994). Selective herbivory and plant community structure in a mid-Atlantic salt marsh. Ecology 75(4):1015-1022.

Gabler, C.A., Osland, M.J., Grace, J.B., Stagg, C.L., Day, R.H., Hartley, S.B., ... McLeod, J.L. (2017). Macroclimatic change expected to transform coastal wetland ecosystems this century. Nature Climate Change 7:142-150. doi: https://doi.org/10.1038/nclimate3203.

Gallai, N., Salles, J.-M., Settele, J. and Vaissière, B.E. (2009). Economic valuation of the vulnerability of world agriculture confronted with pollinator decline. Ecological Economics 68(3):810-821. doi: https://doi.org/10.1016/j.ecolecon.2008.06.014.

Garibaldi, L.A., Steffen-Dewenter, I., Kremen, C., Morales, J.M., Bommarco, R., Cunningham, S.., ... Klein, A.M. (2011). Stability of pollination services decreases with isolation from natural areas despite honey bee visits. Ecology Letters. doi: https://doi.org/10.1111/j.14610248.2011.01669.x.

Garrido, P., Mårell, A., Öckinger, E., Skarin, A., Jansson, A. and Thulin, C.G. (2019). Experimental rewilding enhances grassland functional composition and pollinator habitat use. Journal of Applied Ecology(56):946-955. doi: https://doi.org/10.1111/1365-2664.13338. 
Gascon, C., Brooks, T.M., Contreras-Macbeath, T., Heard, N., Konstant, W., Lamoreux, J., ... Vi??, J.C. (2015). The importance and benefits of species. Current Biology 25(10):R431-R438. doi: https://doi.org/10.1016/j.cub.2015.03.041.

Gedan, K.B., Silliman, B.R. and Bertness, M.D. (2009). Centuries of human-driven change in salt marsh ecosystems. Annual Review of Marine Science 1:117-141. doi: https://doi.org/10.1146/annurev.marine.010908.163930.

Gegear, R.J. and Laverty, T.M. (1998). How many flower types can bumble bees work at the same time? Canadian Journal of Zoology 76(7):1358-1365. doi: https://doi.org/10.1139/z98059.

Ghebreiyessus, Y.T., Gantzer, C.J., Alberts, E.E. and Lentz, R.W. (1994). Soil erosion by concentrated flow: shear stress and bulk density. Transactions of the American Society of Agricultural and Biological Engineers 37(6):1791-1797. doi: https://doi.org/10.13031/2013.28268.

Gittman, R.K. and Keller, D.A. (2013). Fiddler crabs facilitate Spartina alterniflora growth, mitigating periwinkle overgrazing of marsh habitat. Ecology 94(12):2709-2718.

GMEP (2019). Glastir Monitoring and Evaluation Programme: Resources. Available at: https://gmep.wales/resources [Accessed: 26 June 2019].

Good, R.E., Good, N.F. and Frasco, B.R. (1982). A review of primary production and decomposition dynamics of the belowground marsh component. Estuarine Comparisons. Proceedings of the Sixth Biennial International Estuarine Research Conference, Gleneden Beach, Oregon, November 1-6, 1981:139-157. doi: https://doi.org/10.1016/B978-0-12404070-0.50015-6.

Goodloe, R.B., Warren, R.J., Osborn, D.A. and Hall, C. (2000). Population characteristics of feral horses on Cumberland Island, Georgia and their management implications. The Journal of Wildlife Management 64(1):114. doi: https://doi.org/10.2307/3802980.

Gos, P., Loucougaray, G., Colace, M.-P., Arnoldi, C., Gaucherand, S., Dumazel, D., ... Lavorel, S. (2016). Relative contribution of soil, management and traits to co-variations of multiple ecosystem properties in grasslands. Oecologia 180(4):1001-13. doi: https://doi.org/10.1007/s00442-016-3551-3.

Goulson, D., Nicholls, E., Botías, C. and Rotheray, E.L. (2015). Bee declines driven by combined Stress from parasites, pesticides, and lack of flowers. Science 347(6229):1255957. doi: https://doi.org/10.1126/science.1255957.

Gower Salt Marsh Lamb (2019). Gower Salt Marsh Lamb.Pasture For Life. Available at: https://www.pastureforlife.org/profile/B067/ [Accessed: 30 August 2019].

Green, A.J. and Elmberg, J. (2014). Ecosystem services provided by waterbirds. Biological reviews of the Cambridge Philosophical Society 89(1):105-22. doi: https://doi.org/10.1111/brv.12045.

Greenberg, R., Cardoni, A., Ens, B.J., Gan, X., Isacch, J.P., Koffijberg, K. and Loyn, R. (2014). The distribution and conservation of birds of coastal salt marshes. In: Maslo, B. and Lockwood, J. L. (eds.). Coastal Conservation. Cambridge, UK: Cambridge University Press, pp. 180-212.

Greenleaf, S.S., Williams, N.M., Winfree, R. and Kremen, C. (2007). Bee foraging ranges and their relationship to body size. Oecologia 153(3):589-596. doi:

https://doi.org/10.1007/s00442-007-0752-9. 
Griffin, J.N., O'Gorman, E.J., Emmerson, M.C., Jenkins, S.R., Klein, A.M., Loreau, M. and Symstad, A. (2009). Biodiversity and the stability of ecosystem functioning. In: Naeem, S., Bunker, D. E., Hector, A., Loreau, M. and Perrings, C. (eds.). Biodiversity, Ecosystem Functioning, and Human Wellbeing: An Ecological and Economic Perspective. Oxford, UK: Oxford University Press.

Grime, J.P. (1973). Competitive exclusion in herbaceous vegetation. Nature 242:344-347. doi: https://doi.org/10.1038/242344a0.

de Groot, R.S., Alkemade, R., Braat, L., Hein, L. and Willemen, L. (2010). Challenges in integrating the concept of ecosystem services and values in landscape planning, management and decision making. Ecological Complexity 7(3):260-272. doi: https://doi.org/10.1016/j.ecocom.2009.10.006.

De Groot, R.S., Wilson, M.A. and Boumans, R.M.J. (2002). A typology for the classification, description and valuation of ecosystem functions, goods and services. Ecological Economics 41:393-408. doi: https://doi.org/10.1016/S0921-8009(02)00089-7.

Gruenberg, B.U. (2015). The Wild Horse Dilemma: Conflicts and Controversies of the Atlantic Coast Herds. New Providence, PA: Quagga Press.

Haines-Young, R. and Potschin, M. (2010). The links between biodiversity, ecosystem services and human well-being. In: Raffaelli, D. and Frid, C. (eds.). Ecosystem Ecology: A New Synthesis. Cambridge, UK: Cambridge University Press, pp. 110-139.

Haines-Young, R. and Potschin, M.B. (2018). Common international classification of ecosystem services (CICES) V5.1 and guidance on the application of the revised structure.

Halls, L.K. (1970). Nutrient requirements of livestock and game. Range and Wildlife Habitat Evaluation - A Research Symposium. Washington, DC: U.D. Department of Agriculture.

Hanley, N., Breeze, T.D., Ellis, C. and Goulson, D. (2015). Measuring the economic value of pollination services: Principles, evidence and knowledge gaps. Ecosystem Services 14:124132. doi: https://doi.org/10.1016/j.ecoser.2014.09.013.

Hartig, F. (2019). DHARMa: Residual Diagnostics for Hierarchical (Multi-Level / Mixed) Regression Models. R package version 0.2.4.

Harvey, R.J., Garbutt, A., Hawkins, S.J. and Skov, M.W. (2019). No detectable broad-scale effect of livestock grazing on soil blue-carbon stock in salt marshes. Frontiers in Ecology and Evolution 7:151. doi: https://doi.org/10.3389/fevo.2019.00151.

Hay, M.E. and Wells, J.T. (1991). Effects of feral horses on the production, distribution, abundance, and stability of salt-marsh plants: Rachel Carson North Carolina National Estuarine Research Reserve.

Hazelton, E.L.G., Mozdzer, T.J., Burdick, D.M., Kettenring, K.M. and Whigham, D.F. (2014). Phragmites australis management in the United States: 40 years of methods and outcomes. AoB PLANTS 6. doi: https://doi.org/10.1093/aobpla/plu001.

$\mathrm{He}, \mathrm{Q}$. and Silliman, B.R. (2016). Consumer control as a common driver of coastal vegetation worldwide. Ecological Monographs 86(3):278-294. doi: https://doi.org/10.1002/ecm.1221.

Hedges, L. V. and Olkin, I. (1985). Statistical Methods for Meta-Analysis. San Diego: Academic Press.

Hemminga, M.A., Kok, C.J. and de Munck, W. (1988). Decomposition of Spartina anglica roots and rhizomes in a salt marsh of the Westerschelde Estuary. Marine Ecology Progress Series1 48:175-184. 
Henning, C. (2019). fpc: Flexible Procedures for Clustering. R package version 2.2-3.

Hensel, M.J.S. and Silliman, B.R. (2013). Consumer diversity across kingdoms supports multiple functions in a coastal ecosystem. Proceedings of the National Academy of Sciences of the United States of America 110(51):20621-20626. doi: https://doi.org/10.1073/pnas.1312317110.

Hervé, M. (2019). RVAideMemoire: Testing and Plotting Procedures for Biostatistics. R package version 0.9-72.

Hess, G.R., Bartel, R.A., Leidner, A.K., Rosenfeld, K.M., Rubino, M.J., Snider, S.B. and Ricketts, T.H. (2006). Effectiveness of biodiversity indicators varies with extent, grain, and region. Biological Conservation 132(4):448-457. doi: https://doi.org/10.1016/j.biocon.2006.04.037.

Hestbeck, J.B., Nichols, J.D. and Malecki, R.A. (1991). Estimates of movement and site fidelity using mark-resight data of wintering Canada Geese. Ecology 72(2):523-533. doi: https://doi.org/10.2307/2937193.

Heuner, M., Silinski, A., Schoelynck, J., Bouma, T.J., Puijalon, S., Troch, P., ... Temmerman, S. (2015). Ecosystem engineering by plants on wave-exposed intertidal flats is governed by relationships between effect and response traits. PLOS ONE 10(9):e0138086. doi: https://doi.org/10.1371/journal.pone.0138086.

Hobbs, R.J. and Huenneke, L.F. (1992). Disturbance, diversity, and invasion: Implications for conservation. Conservation Biology 6(3):324-337. doi: https://doi.org/10.2307/2386033.

Hobley, E.U., Baldock, J. and Wilson, B. (2016). Environmental and human influences on organic carbon fractions down the soil profile. Agriculture, Ecosystems and Environment 223:152-166. doi: https://doi.org/10.1016/j.agee.2016.03.004.

Holdredge, C., Bertness, M.D. and Altieri, A.H. (2009). Role of crab herbivory in die-off of New England salt marshes. Conservation Biology 23(3):672-679. doi:

https://doi.org/10.1111/j.1523-1739.2008.01137.x.

Holt, A.R., Alix, A., Thompson, A. and Maltby, L. (2016). Food production, ecosystem services and biodiversity: We can't have it all everywhere. Science of The Total Environment 573:1422-1429. doi: https://doi.org/10.1016/j.scitotenv.2016.07.139.

Hoogsteen, M.J.J., Lantinga, E.A., Bakker, E.J., Groot, J.C.J. and Tittonell, P.A. (2015). Estimating soil organic carbon through loss on ignition: Effects of ignition conditions and structural water loss. European Journal of Soil Science 66(2):320-328. doi: https://doi.org/10.1111/ejss.12224.

Howe, C., Suich, H., Vira, B. and Mace, G.M. (2014). Creating win-wins from trade-offs? Ecosystem services for human well-being: A meta-analysis of ecosystem service trade-offs and synergies in the real world. Global Environmental Change 28:263-275. doi: https://doi.org/10.1016/j.gloenvcha.2014.07.005.

Hu, Y., Wang, L., Tang, Y., Li, Y., Chen, J., Xi, X., ... Sun, Y. (2014). Variability in soil microbial community and activity between coastal and riparian wetlands in the Yangtze River estuary Potential impacts on carbon sequestration. Soil Biology and Biochemistry 70:221-228. doi: https://doi.org/10.1016/j.soilbio.2013.12.025.

Hubbard, R.E. and Hansen, R.M. (1976). Diets of wild horses, cattle, and mule deer in the Piceance Basin, Colorado. Journal of Range Management 29(5):389-392. doi: https://doi.org/10.2307/3897147.

Husson, O. (2013). Redox potential (Eh) and pH as drivers of soil/plant/microorganism 
systems: A transdisciplinary overview pointing to integrative opportunities for agronomy. Plant and Soil 362:389-417. doi: https://doi.org/10.1007/s11104-012-1429-7.

Ives, A.R. (2015). For testing the significance of regression coefficients, go ahead and logtransform count data. Methods in Ecology and Evolution 6(7):828-835. doi: https://doi.org/10.1111/2041-210X.12386.

JNCC (2019). UK BAP Broad Habitats. http://jncc.defra.gov.uk/page-5711. Accessed on 16th May 2019.

Johansen, L., Taugourdeau, S., Hovstad, K.A. and Wehn, S. (2019). Ceased grazing management changes the ecosystem services of semi-natural grasslands. Ecosystems and People 15:192-203. doi: https://doi.org/10.1080/26395916.2019.1644534.

Jones, K. and Obiri-Danso, K. (1998). Non-compliance of beaches with the EU directives of bathing water quality: evidence of non-point sources of pollution in Morecambe Bay. Journal of Applied Microbiology Symposium Supplement 85:101S-107S. doi: https://doi.org/10.1111/j.1365-2672.1998.tb05288.x.

Jones, L., Angus, S., Cooper, A., Doody, P., Everard, M., Garbutt, A., ... Whitehouse, A. (2011). Coastal Margins. In: UK National Ecosystem Assessment (ed.). UK National Ecosystem Assessment Technical Report. Cambridge, UK: UNEP-WCMC, pp. 411-458.

Jutila, H.M. (1997). Vascular plant species richness in grazed and ungrazed coastal meadows, SW Finland. Annales Botanici Fennici 34(4):245-263.

Kauffman, J.B., Hernandez Trejo, H., del Carmen Jesus Garcia, M., Heider, C. and Contreras, W. (2015). Carbon stocks of mangroves and losses arising from their conversion to cattle pastures in the Pantanos de Centla, Mexico. Wetlands Ecology and Management 24(2):1-14. doi: https://doi.org/10.1007/s11273-015-9453-z.

Kelleway, J.J., Saintilan, N., Macreadie, P.I. and Ralph, P.J. (2016). Sedimentary factors are key predictors of carbon storage in SE Australian saltmarshes. Ecosystems 19:865-880. doi: https://doi.org/10.1007/s10021-016-9972-3.

Kershaw, H.M. and Mallik, A.U. (2013). Predicting plant diversity response to disturbance: applicability of the Intermediate Disturbance Hypothesis and Mass Ratio Hypothesis. Critical Reviews in Plant Sciences 32(6):383-395. doi: https://doi.org/10.1080/07352689.2013.791501.

Kershaw, S., Campos, C. and Kay, D. (2012). Review of knowledge on the impact of chronic microbial contamination on bivalve shellfish. Cefas/CREH report to Defra, Project WT0923.

Keuskamp, J.A., Dingemans, B.J.J., Lehtinen, T., Sarneel, J.M. and Hefting, M.M. (2013). Tea Bag Index: A novel approach to collect uniform decomposition data across ecosystems. Methods in Ecology and Evolution 4(11):1070-1075. doi: https://doi.org/10.1111/2041210X.12097.

Kim, J.H., Jobbagy, E.G. and Jackson, R.B. (2016). Trade-offs in water and carbon ecosystem services with land-use changes in grasslands. Ecological Applications 26(6):1633-1644.

Kimoto, C., Debano, S.J., Thorp, R.W., Taylor, R. V., Schmalz, H., Delcurto, T., ... Rao, S. (2012). Short-term responses of native bees to livestock and implications for managing ecosystem services in grasslands. Ecosphere 3(10):88. doi: https://doi.org/10.1890/ES12-00118.1.

Kingham, R. (2013). The Broad-Scale Impacts of Livestock Grazing on Saltmarsh Carbon Stocks. Bangor University.

Kirk, W.D.. and Howes, F.. (2012). Plants for Bees. A Guide to the Plants That Benefit the Bees 
of the British Isles. Cardiff, UK: International Bee Research Association.

Kirkpatrick, J.F. and Fazio, P.M. (2010). Wild Horses as Native North American Wildlife.The Science and Conservation Centre, ZooMontana, Billings. Available at:

https://awionline.org/content/wild-horses-native-north-american-wildlife [Accessed: 26 July 2019].

Kirwan, M.L., Temmerman, S., Skeehan, E.E., Guntenspergen, G.R. and Faghe, S. (2016). Overestimation of marsh vulnerability to sea level rise. Nature Climate Change 6(3):253-260. doi: https://doi.org/10.1038/nclimate2909.

van Klink, R., Nolte, S., Mandema, F.S., Lagendijk, G., WallisDeVries, M.F., Bakker, J.P., ... Smit, C. (2016). Effects of grazing management on biodiversity across trophic levels - The importance of livestock species and stocking density in salt marshes. Agriculture, Ecosystems and Environment 235:329-339. doi: https://doi.org/10.1016/j.agee.2016.11.001.

Knapton, S. (2015). Bees contribute more to British economy than Royal family. The Telegraph.

Knowles, J.E. and Frederick, C. (2016). merTools: Tools for analyzing mixed effect regression models 0.2.1.

Koricheva, J., Gurevitch, J. and Mengersen, K. (eds.) (2013). Handbook of Meta-Analysis in Ecology and Evolution. Princeton, New Jersey: Princeton University Press.

Kritzer, J.P., DeLucia, M.-B., Greene, E., Shumway, C., Topolski, M.F., Thomas-Blate, J., ... Smith, K. (2016). The importance of benthic habitats for coastal fisheries. BioScience 66(4):biw014. doi: https://doi.org/10.1093/biosci/biw014.

Kruess, A. and Tscharntke, T. (2002). Grazing intensity and the diversity of grasshoppers, butterflies, and trap-nesting bees and wasps. Conservation Biology 16(6):1570-1580. doi: https://doi.org/10.1046/j.1523-1739.2002.01334.x.

Kuenzler, E.J. (1961). Structure and energy flow of a mussel population in a Georgia salt marsh. Limonology and Oceanography 6(2):191-204.

Kuijper, D.P.J., Beek, P., van Wieren, S.E. and Bakker, J.P. (2008). Time-scale effects in the interaction between a large and a small herbivore. Basic and Applied Ecology 9(2):126-134. doi: https://doi.org/10.1016/j.baae.2006.08.008.

Kuznetsova, A., Brockhoff, P.B. and Christensen, R.H.B. (2017). ImerTest Package: Tests in Linear Mixed Effects Models . Journal of Statistical Software 82(13). doi:

https://doi.org/10.18637/jss.v082.i13.

Ladd, C.J.T., Duggan-Edwards, M.F., Bouma, T.J., Pagès, J.F. and Skov, M.W. Sediment supply explains long-term and large-scale patterns in saltmarsh lateral expansion and erosion. Geophysical Research Letters.

Laffaille, P., Lefeuvre, J. and Feunteun, E. (2000). Impact of sheep grazing on juvenile sea bass, Dicentrarchus labrax L., in tidal salt marshes. Biological Conservation 96(3):271-277. doi: https://doi.org/10.1016/S0006-3207(00)00081-1.

Lagendijk, D.D.G., Howison, R.A., Esselink, P. and Smit, C. (2018). Grazing as a conservation management tool : Responses of voles to grazer species and densities. Basic and Applied Ecology.

Lagendijk, D.D.G., Howison, R.A., Esselink, P., Ubels, R. and Smit, C. (2017). Rotation grazing as a conservation management tool: Vegetation changes after six years of application in a salt marsh ecosystem. Agriculture, Ecosystems and Environment 246:361-366. 
Lal, R. (2008). Carbon sequestration. Philosophical Transactions of the Royal Society $B$ 363:815-830. doi: https://doi.org/10.1098/rstb.2007.2185.

Lambert, R. (2000). Practical management of grazed saltmarshes. In: Sherwood, B. R., Gardiner, B. G. and Harris, T. (eds.). British Saltmarshes. Ceredigion, UK: Forrest Text for The Linnean Society of London, pp. 333-340.

Laursen, K. (1977). The effect of reed harvesting on a population of passerine birds. Dansk Ornitologisk Forenings Tidsskrift 71:95-101.

Layman, C.A. (2000). Fish assemblage structure of the shallow ocean surf-zone on the eastern shore of Virginia Barrier Islands. Estuarine, Coastal and Shelf Science 51(2):201-213. doi: https://doi.org/10.1006/ECSS.2000.0636.

Lázaro, A., Tscheulin, T., Devalez, J., Nakas, G. and Petanidou, T. (2016). Effects of grazing intensity on pollinator abundance and diversity, and on pollination services. Ecological Entomology 41(4):400-412. doi: https://doi.org/10.1111/een.12310.

Lefcheck, J.S. (2016). piecewiseSEM: Piecewise structural equation modelling in $r$ for ecology, evolution, and systematics. Methods in Ecology and Evolution 7(5):573-579. doi: https://doi.org/10.1111/2041-210X.12512.

Lenth, R., Singmann, H., Love, J., Buerkner, P. and Herve, M. (2019). Package 'emmeans'. Estimated Marginal Means, aka Least-Squares Means.

Levin, P.S., Ellis, J., Petrik, R. and Hay, M.E. (2002). Indirect effects of feral horses on estuarine communities. Conservation Biology 16(5):1364-1371.

Lin, S., Wu, R., Yang, F., Wang, J. and Wu, W. (2018). Spatial trade-offs and synergies among ecosystem services within a global biodiversity hotspot. Ecological Indicators 84:371-381. doi: https://doi.org/10.1016/j.ecolind.2017.09.007.

Loomis, M.J. and Craft, C.B. (2010). Carbon sequestration and nutrient (Nitrogen, Phosphorus) accumulation in river-dominated tidal marshes, Georgia, USA. Soil Science Society of America Journal 74(3):1028-1036. doi: https://doi.org/10.2136/sssaj2009.0171.

van Loon-Steensma, J.M. and Vellinga, P. (2013). Trade-offs between biodiversity and flood protection services of coastal salt marshes. Current Opinion in Environmental Sustainability 5(3-4):320-326. doi: https://doi.org/10.1016/j.cosust.2013.07.007.

Lüdecke, D. (2019). sjsstats: Statistical functions for regression models. R package version 0.17.4.

Lunt, I.D., Eldridge, D.J., Morgan, J.W. and Witt, G.B. (2007). A framework to predict the effects of livestock grazing and grazing exclusion on conservation values in natural ecosystems in Australia. Australian Journal of Botany 55:401-415. doi: https://doi.org/10.1071/BT06178.

MA (2005a). Ecosystems and Human Well-Being: Synthesis. Washington, DC: Island Press.

MA (2005b). Food. Ecosystems and Human Well-Being: Current State and Trends Assessment. Washington, DC: Island Press, pp. 209-241.

MA (2005c). Coastal systems. In: Hassan, R., Scholes, R. and Ash, N. (eds.). Ecosystems and Human Well-Being: Current State and Trends, Volume 1. Washington, DC: Island Press.

Mace, G.M., Bateman, I., Albon, S., Balmford, A., Brown, C., Church, A., ... Winn, J. (2011). Conceptual Framework and Methodology. In: UK National Ecosystem Assessment (ed.). UK National Ecosystem Assessment Technical Report. Cambridge, UK: UNEP-WCMC, pp. 11-26. 
MacKenzie, R.A. and Dionne, M. (2008). Habitat heterogeneity: Importance of salt marsh pools and high marsh surfaces to fish production in two Gulf of Maine salt marshes. Marine Ecology Progress Series 368:217-230. doi: https://doi.org/10.3354/meps07560.

Macreadie, P.I., Ollivier, Q.R., Kelleway, J.J., Serrano, O., Carnell, P.E., Ewers Lewis, C.J., ... Lovelock, C.E. (2017). Carbon sequestration by Australian tidal marshes. Scientific Reports 7(1):44071. doi: https://doi.org/10.1038/srep44071.

Maes, J., Egoh, B., Willemen, L., Liquete, C., Vihervaara, P., Schägner, J.P., ... Bidoglio, G. (2012). Mapping ecosystem services for policy support and decision making in the European Union. Ecosystem Services 1(2012):31-39. doi: https://doi.org/10.1016/j.ecoser.2012.06.004.

Malpas, L.R., Smart, J., Drewitt, A., Sharps, E. and Garbutt, A. (2013). Continued declines of Redshank Tringa totanus breeding on saltmarsh in Great Britain: is there a solution to this conservation problem? Bird Study 60(3):370-383. doi: https://doi.org/10.1080/00063657.2013.781112.

Manning, P., Van Der Plas, F., Soliveres, S., Allan, E., Maestre, F.T., Mace, G., ... Fischer, M. (2018). Redefining ecosystem multifunctionality. Nature Ecology and Evolution 2(3):427-436. doi: https://doi.org/10.1038/s41559-017-0461-7.

Martín-López, B., Iniesta-Arandia, I., García-Llorente, M., Palomo, I., Casado-Arzuaga, I., Amo, D.G. Del, ... Montes, C. (2012). Uncovering ecosystem service bundles through social preferences Bawa, K. (ed.). PLOS ONE 7(6):e38970. doi: https://doi.org/10.1371/journal.pone.0038970.

Mason, L.R., Feather, A., Godden, N., Vreugdenhil, C.C. and Smart, J. (2019). Are agrienvironment schemes successful in delivering conservation grazing management on saltmarsh? Journal of Applied Ecology.

Mastrangelo, M.E., Weyland, F., Villarino, S.H., Barral, M.P., Nahuelhual, L. and Laterra, P. (2014). Concepts and methods for landscape multifunctionality and a unifying framework based on ecosystem services. Landscape Ecology 29(2):345-358. doi: https://doi.org/10.1007/s10980-013-9959-9.

McCraith, B.J., Gardner, L.R., Wethey, D.S. and Moore, W.S. (2003). The effect of fiddler crab burrowing on sediment mixing and radionuclide profiles along a topographic gradient in a southeastern salt marsh. Journal of Marine Research 61(3):359-390. doi: https://doi.org/10.1357/002224003322201232.

McKee, K.L., Mendelssohn, I.A. and D. Materne, M. (2004). Acute salt marsh dieback in the Mississippi River deltaic plain: a drought-induced phenomenon? Global Ecology and Biogeography 13(1):65-73. doi: https://doi.org/10.1111/j.1466-882X.2004.00075.x.

McKinley, E., Ballinger, R.C. and Beaumont, N.J. (2018). Saltmarshes, ecosystem services, and an evolving policy landscape: A case study of Wales, UK. Marine Policy 91.

McKinley, E., Pagès, J.F., Wyles, K.J. and Beaumont, N. (2019). Ecosystem services: A bridge or barrier for UK marine stakeholders? Ecosystem Services 37:100922. doi: https://doi.org/10.1016/J.ECOSER.2019.100922.

McLeod, E., Chmura, G.L., Bouillon, S., Salm, R., Bjork, M., Duarte, C.M., ... Silliman, B.R. (2011). A blueprint for blue carbon: toward an improved understanding of the role of vegetated coastal habitats in sequestering $\mathrm{CO} 2$. Frontiers in Ecology and The Environment 9:552-560.

McOwen, C., Weatherdon, L., Bochove, J.-W., Sullivan, E., Blyth, S., Zockler, C., ... Fletcher, S. 
(2017). A global map of saltmarshes. Biodiversity Data Journal 5:e11764. doi: https://doi.org/10.3897/bdj.5.e11764.

McSherry, M.E. and Ritchie, M.E. (2013). Effects of grazing on grassland soil carbon: a global review. Global Change Biology 19(5):1347-57. doi: https://doi.org/10.1111/gcb.12144.

Meirland, A., Bouvet, A., Rybarczyk, H., Dubois, F. and Chabrerie, O. (2013). Effects of sheep grazing on salt-marsh plant communities in the bay of somme (France). Revue d'Ecologie ( $L a$ Terre et la Vie) 68:319-333.

Memmott, J. (1999). The structure of a plant-pollinator food web. Ecology Letters 2(5):276280. doi: https://doi.org/10.1046/j.1461-0248.1999.00087.x.

Meyer, H., Fock, H., Haase, A., Reinke, H.D. and Tulowitzki, I. (1995). Structure of the invertebrate fauna in salt marshes of the Wadden Sea coast of Schleswig-Holstein influenced by sheep-grazing. Helgoländer Meeresuntersuchungen 49:563-589. doi: https://doi.org/10.1007/BF02368383.

Meyer, S.T., Ebeling, A., Eisenhauer, N., Hertzog, L., Hillebrand, H., Milcu, A., ... Weisser, W.W. (2016). Effects of biodiversity strengthen over time as ecosystem functioning declines at low and increases at high biodiversity. Ecosphere 7(12):e01619. doi: https://doi.org/10.1002/ecs2.1619.

Mitsch, W.J. and Gosselink, J.G. (2008). Wetlands. New York, USA: Van Nostrand Reinhold.

Möller, I., Kudella, M., Rupprecht, F., Spencer, T., Paul, M., van Wesenbeeck, B.K., ... Schimmels, S. (2014). Wave attenuation over coastal salt marshes under storm surge conditions. Nature Geoscience 7(10):727-731. doi: https://doi.org/10.1038/ngeo2251.

Möller, I., Spencer, T., French, J.R., Leggett, D.J. and Dixon, M. (1999). Wave transformation over salt marshes: A field and numerical modelling study from north Norfolk, England. Estuarine, Coastal and Shelf Science 49:411-426. doi: https://doi.org/10.1006/ecss.1999.0509.

Monbiot, G. (2014). Put a price on nature? We must stop this neoliberal road to ruin. The Guardian.

Mu, J., Zeng, Y., Wu, Q., Niklas, K.J. and Niu, K. (2016). Traditional grazing regimes promote biodiversity and increase nectar production in Tibetan alpine meadows. Agriculture, Ecosystems and Environment 233:336-342. doi: https://doi.org/10.1016/j.agee.2016.09.030.

Mudd, S.M., D'Alpaos, A. and Morris, J.T. (2010). How does vegetation affect sedimentation on tidal marshes? Investigating particle capture and hydrodynamic controls on biologically mediated sedimentation. Journal of Geophysical Research 115(F3):F03029. doi: https://doi.org/10.1029/2009JF001566.

Murphy, S., Jordan, P., Mellander, P.-E. and O' Flaherty, V. (2015). Quantifying faecal indicator organism hydrological transfer pathways and phases in agricultural catchments. Science of The Total Environment 520:286-299. doi: https://doi.org/10.1016/J.SCITOTENV.2015.02.017.

Murray, B.C., Pendleton, L., Jenkins, W.A. and Sifleet, S. (2011). Green Payments for Blue Carbon: Economic Incentives for Protecting Threatened Coastal Habitats. Durham, North Carolina.

Murray, T.E., Fitzpatrick, Ú., Byrne, A., Fealy, R., Brown, M.J.F. and Paxton, R.J. (2012). Localscale factors structure wild bee communities in protected areas. Journal of Applied Ecology 49(5):998-1008. doi: https://doi.org/10.1111/j.1365-2664.2012.02175.x. 
Narayan, S., Beck, M.W., Reguero, B.G., Losada, I.J., van Wesenbeeck, B., Pontee, N., ... Burks-Copes, K.A. (2016). The effectiveness, costs and coastal protection benefits of natural and nature-based defences. PloS one 11(5):e0154735. doi:

https://doi.org/10.1371/journal.pone.0154735.

National Park Service (1991). Natural Resource Management Reference Manual \#77. NPS-77. Washington DC.

National Park Service (2014). Foundation Document: Cumberland Island National Seashore, Georgia.

National Park Service (2019). Ameliorating Salt Marsh Vegetation Losses.National Park Service. Cape Cod National Seashore. Available at:

https://www.nps.gov/caco/learn/nature/revegetation-of-salt-marsh-dieback-areas.htm [Accessed: 29 August 2019].

Natural England (2009). Grazing livestock in the lowlands. Environmental Impacts of Land Management. Natural England Research Report NERR030, pp. 62-75.

Newton, A.C., Stewart, G.B., Myers, G., Diaz, A., Lake, S., Bullock, J.M. and Pullin, A.S. (2009). Impacts of grazing on lowland heathland in north-west Europe. Biological Conservation 142(5):935-947. doi: https://doi.org/10.1016/j.biocon.2008.10.018.

Nilsson, L., Andersson, G.K.S., Birkhofer, K. and Smith, H.G. (2017). Ignoring ecosystemservice cascades undermines policy for multifunctional agricultural landscapes. Frontiers in Ecology and Evolution 5:109. doi: https://doi.org/10.3389/fevo.2017.00109.

Nolte, S., Koppenaal, E.C., Esselink, P., Dijkema, K.S., Schuerch, M., De Groot, A. V., ... Temmerman, S. (2013a). Measuring sedimentation in tidal marshes: A review on methods and their applicability in biogeomorphological studies. Journal of Coastal Conservation 17(3):301-325. doi: https://doi.org/10.1007/s11852-013-0238-3.

Nolte, S., Mueller, F., Schuerch, M., Wanner, A., Esselink, P., Bakker, J.P. and Jensen, K. (2013b). Does livestock grazing affect sediment deposition and accretion rates in salt marshes? Estuarine Coastal and Shelf Science 135:296-305. doi: https://doi.org/10.1016/j.ecss.2013.10.026.

Norris, K. (2000). The conservation and management of saltmarshes for birds. In: Sherwood, B. R., Gardiner, B. G. and Harris, T. (eds.). British Saltmarshes. Ceredigion, UK: Forrest Text for The Linnean Society of London, pp. 341-358.

O'Rourke, E. and Kramm, N. (2012). High nature value (HNV) farming and the management of upland diversity. A review. European Countryside 4(2):116-133. doi:

https://doi.org/10.2478/v10091-012-0018-3.

Oksanen, J., Blanchet, F.G., Friendly, M., Kindt, R., Legendre, P., McGlinn, D., ... Wagner, H. (2019). vegan: Community Ecology Package. R package version 2.5-4.

Oliver, T.H., Heard, M.S., Isaac, N.J.B., Roy, D.B., Procter, D., Eigenbrod, F., ... Bullock, J.M. (2015). Biodiversity and resilience of ecosystem functions. Trends in Ecology \& Evolution 30(11):673-684. doi: https://doi.org/10.1016/j.tree.2015.08.009.

Olsen, Y.S., Dausse, A., Garbutt, A., Ford, H., Thomas, D.N. and Jones, D.L. (2011). Cattle grazing drives nitrogen and carbon cycling in a temperate salt marsh. Soil Biology and Biochemistry 43(3):531-541. doi: https://doi.org/10.1016/j.soilbio.2010.11.018.

Osborne, C.P., Salomaa, A., Kluyver, T. a, Visser, V., Kellogg, E. a, Morrone, O., ... Simpson, D. a (2014). A global database of C4 photosynthesis in grasses. New Phytologist 204(3):441- 
446. doi: https://doi.org/10.1111/nph.12942.

Van Oudenhoven, A.P.E., Petz, K., Alkemade, R., Hein, L. and De Groot, R.S. (2012).

Framework for systematic indicator selection to assess effects of land management on ecosystem services. Ecological Indicators 21:110-122. doi:

https://doi.org/10.1016/j.ecolind.2012.01.012.

Ouyang, X. and Lee, S.Y. (2014). Updated estimates of carbon accumulation rates in coastal marsh sediments. Biogeosciences 11(18):5057-5071. doi: https://doi.org/10.5194/bg-115057-2014.

Owen, M. (1990). The damage-conservation interface illustrated by geese. Ibis 132(2):238252. doi: https://doi.org/10.1111/j.1474-919X.1990.tb01042.x.

Pagès, J.F., Jenkins, S.R., Bouma, T.J., Sharps, E. and Skov, M.W. (2018). Opposing indirect effects of domestic herbivores on saltmarsh erosion. Ecosystems. doi: https://doi.org/10.1007/s10021-018-0322-5.

De Palma, A., Kuhlmann, M., Roberts, S.P.M., Potts, S.G., Börger, L., Hudson, L.N., ... Purvis, A. (2015). Ecological traits affect the sensitivity of bees to land-use pressures in European agricultural landscapes. Journal of Applied Ecology 52(6):1567-1577. doi:

https://doi.org/10.1111/1365-2664.12524.

Pardoe, I. (2012). Applied Regression Modeling. 2nd ed. New Jersey: John Wiley \& Sons, Inc.

Paul, M., Rupprecht, F., Moller, I., Bouma, T.J., Spencer, T., Kudella, M., ... Schimmels, S.

(2016). Plant stiffness and biomass as drivers for drag forces under extreme wave loading: A flume study on mimics. Coastal Engineering 117:70-78. doi:

https://doi.org/10.1016/j.coastaleng.2016.07.004.

Pauls, L. (2017). Carmarthen Bay and Estuaries SAC Atlantic Salt Meadows Monitoring Report.

Paz-Kagan, T., Ohana-Levi, N., Herrmann, I., Zaady, E., Henkin, Z. and Karnieli, A. (2016). Grazing intensity effects on soil quality: A spatial analysis of a Mediterranean grassland. Catena. doi: https://doi.org/10.1016/j.catena.2016.04.020.

Perry, S. (2016). The Essex Serpent. London: Serpent's Tail.

Petz, K., Alkemade, R., Bakkenes, M., Schulp, C.J.E., van der Velde, M. and Leemans, R. (2014). Mapping and modelling trade-offs and synergies between grazing intensity and ecosystem services in rangelands using global-scale datasets and models. Global Environmental Change 29:223-234. doi: https://doi.org/10.1016/j.gloenvcha.2014.08.007.

Pidgeon, E. (2009). Carbon sequestration by coastal marine habitats: Important missing sinks. In: Laffoley, D. d'A. and Grimsditch, G. (eds.). The Management of Natural Coastal Carbon Sinks. Gland, Switzerland: IUCN, pp. 47-51.

Pollard, E. and Yates, T.. (1993). Monitoring Butterflies for Ecology and Conservation. London: Chapman \& Hall.

Potschin, M.B., Primmer, E., Furman, E. and Haines-young, R.H. (2016). Have Ecosystem Services Been Oversold? A Response to Silvertown. Trends in Ecology \& Evolution 2090:1-2. doi: https://doi.org/10.1016/j.tree.2016.03.008.

Potts, S.G., Biesmeijer, J.C., Kremen, C., Neumann, P., Schweiger, O. and Kunin, W.E. (2010). Global pollinator declines: Trends, impacts and drivers. Trends in Ecology and Evolution 25(6):345-353. doi: https://doi.org/10.1016/j.tree.2010.01.007. 
Potts, S.G., Roberts, S.P.M., Dean, R., Marris, G., Brown, M.A., Jones, R., ... Settele, J. (2010). Declines of managed honey bees and beekeepers in Europe. Journal of Apicultural Research 49(1):15-22. doi: https://doi.org/10.3896/IBRA.1.49.1.02.

Potts, S.G., Vulliamy, B., Roberts, S., O'Toole, C., Dafni, A., Ne'eman, G. and Willmer, P.G. (2004). Nectar resource diversity organises flower-visitor community structure. Entomologia Experimentalis et Applicata 113(2):103-107. doi: https://doi.org/10.1111/j.0013-

8703.2004.00212.x.

Powney, G.D., Carvell, C., Edwards, M., Morris, R.K.A., Roy, H.E., Woodcock, B.A. and Isaac, N.J.B. (2019). Widespread losses of pollinating insects in Britain. Nature Communications 10(1):1-6. doi: https://doi.org/10.1038/s41467-019-08974-9.

Prince, R. (2011). Tried and tested: salt marsh lamb. The Telegraph.

Pullin, A.S. and Stewart, G.B. (2006). Guidelines for systematic review in conservation and environmental management. Conservation Biology 20(6):1647-1656. doi: https://doi.org/10.1111/j.1523-1739.2006.00485.x.

Quirk, M. (2002). Managing grazing. In: Grice, A. C. and Hodgkinson, K. C. (eds.). Global Rangelands. Progress and Prospects. Wallingford, UK: CABI Publishing in association with Center for International Forestry Research (CIFOR), pp. 131-146.

R Core Team (2014). R: A language and environment for statistical computing. R Foundation for Statistical Computing, Vienna, Austria. http://www.R-project.org/.

R Core Team (2018). R: A language and environment for statistical computing. R Foundation for Statistical Computing, Vienna, Austria. http://www.R-project.org/.

Rannap, R., Kaart, T., Pehlak, H., Kana, S., Soomets, E. and Lanno, K. (2017). Coastal meadow management for threatened waders has a strong supporting impact on meadow plants and amphibians. Journal for Nature Conservation 35:77-91. doi:

https://doi.org/10.1016/j.jnc.2016.12.004.

Ranwell, D. (1961). Spartina salt marshes in southern England: I. The effects of sheep grazing at the upper limits of Spartina marsh in Bridgwater Bay. Journal of Ecology 49(325-340).

Rassweiler, A., Costello, C., Hilborn, R. and Siegel, D.A. (2014). Integrating scientific guidance into marine spatial planning. Proceedings of the Royal Society B: Biological Sciences 281(1781):20132252. doi: https://doi.org/10.1098/rspb.2013.2252.

Raudsepp-Hearne, C., Peterson, G.D. and Bennett, E.M. (2010). Ecosystem service bundles for analyzing tradeoffs in diverse landscapes. Proceedings of the National Academy of Sciences 107(11):5242-5247. doi: https://doi.org/10.1073/pnas.0907284107.

Reader, J. and Craft, C. (1999). Comparison of wetland structure and function on grazed and ungrazed salt marshes. Journal of the Elisha Mitchell Scientific Society 115(4):236-249.

Reimold, R.J., Linthurst, R.A. and Wolf, P.L. (1975). Effects of grazing on a salt marsh. Biological Conservation 8(2):105-125. doi: https://doi.org/10.1016/0006-3207(75)90036-1.

Ren, H., Eviner, V.T., Gui, W., Wilson, G.W.T., Cobb, A.B., Yang, G., ... Bai, Y. (2018). Livestock grazing regulates ecosystem multifunctionality in semi-arid grassland. Functional Ecology 32(12):2790-2800. doi: https://doi.org/10.1111/1365-2435.13215.

Renard, D., Rhemtulla, J.M. and Bennett, E.M. (2015). Historical dynamics in ecosystem service bundles. Proceedings of the National Academy of Sciences:201502565. doi: https://doi.org/10.1073/pnas.1502565112. 
Ribeiro, H. and Mucha, A.P. (2011). Hydrocarbon degradation potential of salt marsh plantmicroorganisms associations. Biodegradation:729-739. doi: https://doi.org/10.1007/s10532010-9446-9.

Rickert, C., Fichtner, A. and van Klink, R. (2018). Livestock grazing disrupts plant-insect interactions on salt marshes. Insect Conservation and Diversity 11:152-161. doi: https://doi.org/10.1111/icad.12251.

Rickert, C., Fichtner, A., van Klink, R. and Bakker, J.P. (2012). Alpha- and beta-diversity in moth communities in salt marshes is driven by grazing management. Biological Conservation 146(1):24-31. doi: https://doi.org/10.1016/j.biocon.2011.11.024.

Ricketts, T.H., Regetz, J., Steffan-Dewenter, I., Cunningham, S.A., Kremen, C., Bogdanski, A., ... Viana, B.F. (2008). Landscape effects on crop pollination services: Are there general patterns? Ecology Letters 11(5):499-515. doi: https://doi.org/10.1111/j.1461-

0248.2008.01157.x.

Rodríguez, J.P., Beard, T.D., Bennett, E.M., Cumming, G.S., Cork, S.J., Agard, J., ... Peterson, G.D. (2006). Trade-offs across space, time, and ecosystem services. Ecology and Society 11(1):28. doi: https://doi.org/10.5751/ES-01667-110128.

Rodwell, J.S. (2006). NVC Users' Handbook. Peterborough, UK: Joint Nature Conservation Committee.

Roman, C.T. and Burdick, D.M. (eds.) (2012). Tidal Marsh Restoration :A Synthesis of Science and Management. Washington, DC: Island Press.

Rook, A.J., Dumont, B., Isselstein, J., Osoro, K., WallisDeVries, M.F., Parente, G. and Mills, J. (2004). Matching type of livestock to desired biodiversity outcomes in pastures - a review. Biological Conservation 119(2):137-150. doi: https://doi.org/10.1016/j.biocon.2003.11.010.

Root-Bernstein, M. and Jaksic, F.M. (2017). Making research relevant? Ecological methods and the ecosystem services framework. Earth's Future 5:664-678. doi:

https://doi.org/10.1002/eft2.222.

Rowland, C.S., Morton, R.D., Carrasco, L., McShane, G., O'Neil, A.W. and Wood, C.M. (2017). Land Cover Map 2015 (vector, GB).

RSPB (2018). Bird A-Z. Available at: https://www.rspb.org.uk/birds-and-wildlife/wildlifeguides/bird-a-z/ [Accessed: 29 December 2017].

Ryan, J. (2011). Anthoethnography: Emerging research into the culture of flora, aesthetic experience of plants, and the wildflower tourism of the future. New Scholar 1(1):28-40.

Scholte, S.S.K., Todorova, M., van Teeffelen, A.J.A. and Verburg, P.H. (2016). Public support for wetland restoration: what is the link with Ecosystem Service values? Wetlands 36:467481. doi: https://doi.org/10.1007/s13157-016-0755-6.

Schrama, M., Heijning, P., Bakker, J.P., van Wijnen, H.J., Berg, M.P. and Olff, H. (2013). Herbivore trampling as an alternative pathway for explaining differences in nitrogen mineralization in moist grasslands. Oecologia 172:231-243. doi:

https://doi.org/10.1007/s00442-012-2484-8.

Schuerch, M., Spencer, T., Temmerman, S., Kirwan, M.L., Wolff, C., Lincke, D., ... Brown, S. (2018). Future response of global coastal wetlands to sea-level rise. Nature 561:231-234. doi: https://doi.org/10.1038/s41586-018-0476-5.

Scottish Government (2016). Information on Livestock Units. Available at: http://www.gov.scot/Topics/farmingrural/Agriculture/grants/Schemes/LMCMS/Options/Info 
/LivestockInfo [Accessed: 2 February 2016].

Seddon, N., Mace, G., Naeem, S., Pigot, A., Mouillot, D., Cavanagh, R.D., ... Vause, J. (2016). Biodiversity in the Anthropocene: prospects and policy. Proceedings of the Royal Society $B$ : Biological Sciences 283:20162094. doi: https://doi.org/10.1098/rspb.2016.2094.

Sedgwick, P. (2013). Meta-analyses: how to read a funnel plot. BMJ 346:f1342. doi: https://doi.org/http://dx.doi.org/10.1136/bmj.f1342.

Sharp, S.J. and Angelini, C. (2016). Whether disturbances alter salt marsh soil structure dramatically affects Spartina alterniflora recolonization rate. Ecosphere 7(11):1-16. doi: https://doi.org/10.1002/ecs2.1540.

Sharp, S.J. and Angelini, C. (2019). The role of landscape composition and disturbance type in mediating salt marsh resilience to feral hog invasion. Biological Invasions 21(9):2857-2869. doi: https://doi.org/10.1007/s10530-019-02018-5.

Sharps, E., Smart, J., Mason, L.R., Jones, K., Skov, M.W., Garbutt, A. and Hiddink, J.G. (2017). Nest trampling and ground nesting birds: Quantifying temporal and spatial overlap between cattle activity and breeding redshank. Ecology and Evolution 7(16):6622-6633. doi: https://doi.org/10.1002/ece3.3271.

Sharps, E., Smart, J., Skov, M.W., Garbutt, A. and Hiddink, J.A.N.G. (2015). Light grazing of saltmarshes is a direct and indirect cause of nest failure in Common Redshank Tringa totanus. Ibis 157:239-249.

Shepard, C.C., Crain, C.M. and Beck, M.W. (2011). The protective role of coastal marshes: a systematic review and meta-analysis. PloS one 6(11):e27374. doi: https://doi.org/10.1371/journal.pone.0027374.

Sherr, E.B. (2015). Marsh Mud and Mummichogs: An Intimate Natural History of Coastal Georgia. Athens, GA, USA: University of Georgia Press.

Silliman, B.R. and Bertness, M.D. (2002). A trophic cascade regulates salt marsh primary production. Proceedings of the National Academy of Sciences of the United States of America 99(16):10500-5. doi: https://doi.org/10.1073/pnas.162366599.

Silliman, B.R., van de Koppel, J., Bertness, M.D., Stanton, L.E. and Mendelssohn, I.A. (2005). Drought, snails, and large-scale die-off of southern U.S. salt marshes. Science (New York, N.Y.) 310(5755):1803-6. doi: https://doi.org/10.1126/science.1118229.

Silliman, B.R. and Newell, S.Y. (2003). Fungal farming in a snail. Proceedings of the National Academy of Sciences of the United States of America 100(26):15643-8. doi: https://doi.org/10.1073/pnas.2535227100.

Silvertown, J. (2015). Have ecosystem services been oversold? Trends in Ecology \& Evolution 30(11):641-648. doi: https://doi.org/10.1016/j.tree.2015.08.007.

Skov, M.W., Ford, H., Webb, J., Kayoueche-Reeve, M., Hockley, N., Paterson, D. and Garbutt, A. (2016). The Saltmarsh Carbon Stock Predictor: A Tool for Predicting Carbon Stocks of Welsh and English Salt Marshes, UK. Bangor University, UK, UK: CBESS, Biodiversity and Ecosystem Service Sustainability programme (NERC NE/J015350/1).

Small, N., Munday, M. and Durance, I. (2017). The challenge of valuing ecosystem services that have no material benefits. Global Environmental Change 44:57-67. doi: https://doi.org/10.1016/j.gloenvcha.2017.03.005.

Smith, D.C., Konrad, V., Koulouris, H., Borns, E.H. and H.W. and Jr. (1989). Salt marshes as a factor in the agriculture of northeastern North America. Agricultural History 63(2):270-294. 
doi: https://doi.org/10.2307/3743517.

Soil Classification Working Group (1998). Terminology for Describing Soils. The Canadian System of Soil Classification. 3rd editio. Agriculture and Agri-Food Canada Publication, 187 pp.

Soliveres, S., Manning, P., Prati, D., Gossner, M.M., Alt, F., Arndt, H., ... Allan, E. (2016). Locally rare species influence grassland ecosystem multifunctionality. Philosophical Transactions of the Royal Society B: Biological Sciences 371(1694):20150269. doi: https://doi.org/10.1098/rstb.2015.0269.

Soomdat, N.N., Griffin, J.N., McCoy, M., Hensel, M.J.S., Buhler, S., Chejanovski, Z. and Silliman, B.R. (2014). Independent and combined effects of multiple predators across ontogeny of a dominant grazer. Oikos 123(9):1081-1090. doi:

https://doi.org/10.1111/oik.01579.

Southorn, N. and Cattle, S. (2004). The dynamics of soil quality in livestock grazing systems. SuperSoil 2004: 3rd Australian New Zealand Soils Conference, 5-9 December 2004. University of Sydney, Australia.

Spake, R., Lasseur, R., Crouzat, E., Bullock, J.M., Lavorel, S., Parks, K.E., ... Eigenbrod, F. (2017). Unpacking ecosystem service bundles: Towards predictive mapping of synergies and trade-offs between ecosystem services. Global Environmental Change 47:37-50. doi: https://doi.org/10.1016/j.gloenvcha.2017.08.004.

Steffan-Dewenter, I., Munzenberg, U., Burger, C., Thies, C. and Tscharntke, T. (2002). Scaledependent effects of landscape context on three pollinator guilds. Ecology 83(5):1421-1432.

Sterne, J.A.C. and Egger, M. (2001). Funnel plots for detecting bias in meta-analysis: Guidelines on choice of axis. Journal of Clinical Epidemiology 54(10):1046-1055. doi: https://doi.org/10.1016/S0895-4356(01)00377-8.

Stevens, B.F. (1986). The Feral Ponies of the Rachel Carson Estuarine Sanctuary. A Management Study.

Stewart, G.B. and Pullin, A.S. (2008). The relative importance of grazing stock type and grazing intensity for conservation of mesotrophic 'old meadow' pasture. Journal for Nature Conservation 16(3):175-185. doi: https://doi.org/10.1016/j.jnc.2008.09.005.

Stigner, M.G., Beyer, H.L., Klein, C.J. and Fuller, R.A. (2016). Reconciling recreational use and conservation values in a coastal protected area. Journal of Applied Ecology. doi: https://doi.org/10.1111/1365-2664.12662.

Taggart, J.B. (2008). Management of feral horses at the North Carolina National Estuarine Research Reserve. Natural Areas Journal 28(2):187-195. doi: https://doi.org/10.3375/08858608(2008)28[187:MOFHAT]2.0.CO;2.

Tanentzap, A.J. and Coomes, D.A. (2012). Carbon storage in terrestrial ecosystems: do browsing and grazing herbivores matter? Biological reviews of the Cambridge Philosophical Society 87(1):72-94. doi: https://doi.org/10.1111/j.1469-185X.2011.00185.x.

Taubert, R.B. and Murphy, K.J. (2012). Long-term dynamics in Scottish saltmarsh plant communities. The Glasgow Naturalist 25(4):111-118.

Teal, J.M. (1962). Energy flow in the salt marsh ecosystem of Georgia. Ecology 43(4):614624.

TEEB (2019). Ecosystem Services. The Economics of Ecosystems \& Biodiversity. Available at: http://www.teebweb.org/resources/ecosystem-services/ [Accessed: 4 September 2019]. 
Thien, S. (1979). A flow diagram for teaching texture-by-feel analysis. Journal of Agronomic Education 8:54-55.

Timberlake, T.P., Vaughan, I.P. and Memmott, J. (2019). Phenology of farmland floral resources reveals seasonal gaps in nectar availability for bumblebees. Journal of Applied Ecology:preprint. doi: https://doi.org/10.1111/1365-2664.13403.

Török, P., Penksza, K., Tóth, E., Kelemen, A., Sonkoly, J. and Tóthmérész, B. (2018). Vegetation type and grazing intensity jointly shape grazing effects on grassland biodiversity. Ecology and Evolution 8(20):10326-10335. doi: https://doi.org/10.1002/ece3.4508.

Tscharntke, T. (1997). Vertebrate effects on plant-invertebrate food webs. In: Gange, A. C. and Brown, V. K. (eds.). Multitrophic Interactions in Terrestrial Systems. Oxford, UK: Backwell Science, pp. 277-298.

Turner, K.G., Odgaard, M.V., Bøcher, P.K., Dalgaard, T. and Svenning, J.-C. (2014). Bundling ecosystem services in Denmark: Trade-offs and synergies in a cultural landscape. Landscape and Urban Planning 125:89-104. doi: https://doi.org/10.1016/j.landurbplan.2014.02.007.

Turner, M.G. (1987). Effects of grazing by feral horses, clipping, trampling, and burning on a Georgia salt marsh. Estuaries 10(1):54-60. doi: https://doi.org/10.2307/1352025.

Turner, M.G. (1988). Simulation and management implications of feral horse grazing on Cumberland Island, Georgia. Journal of Range Management 41(5):441-447. doi: https://doi.org/10.2307/3899586.

Turner, R.E., Swenson, E.M. and Milan, C.S. (2002). Organic and inorganic contributions to vertical accretion in salt marsh sediments Weinstein, M. P. and Kreeger, D. A. (eds.). Concepts and Controversies in Tidal Marsh Ecology 30(11):583-595. doi: https://doi.org/10.1007/0-306-47534-0_27.

UKBMS (2019). Wider Countryside Butterfly Survey.United Kingdom Butterfly Monitoring Scheme. Available at: http://www.ukbms.org/wcbs [Accessed: 24 July 2019].

United Nations (2019). World Population Prospects 2019: Highlights.

USDA (2017). Guide to Texture by Feel.United States Department of Agriculture, Natural Resources Conservation Science. Available at: https://www.nrcs.usda.gov/wps/portal/nrcs/detail/soils/edu/?cid=nrcs142p2_054311 [Accessed: 10 September 2017].

Valdes, K., Hunter, E.A. and Nibbelink, N.P. (2016). Salt marsh elevation is a strong determinant of nest-site selection by Clapper Rails in Georgia, USA. Journal of Field Ornithology 87(1):65-73. doi: https://doi.org/10.1111/jofo.12134.

VanderMeer, J. (2014). Annihilation. New York, USA: Farrar, Strauss and Giroux.

Vernberg, F.J. (1993). Saltmarsh processes: A review. Environmental Toxicology and Chemistry 12:2167-2195. doi: https://doi.org/10.1002/etc.5620121203.

Viana, B.F., Boscolo, D., Neto, E.M., Lopes, L.E., Lopes, A. V, Ferreira, P.A., ... Primo, L.M. (2012). How well do we understand landscape effects on pollinators and pollination services? Journal of Pollination Ecology 7(5):31-41. doi:

https://doi.org/10.1098/rspb.2006.3721.

Viechtbauer, W. (2010). Conducting meta-analyses in R with the metafor package. Journal of Statistical Software 36(3):1-48.

de Vlas, J., Mandema, F., Nolte, S., van Klink, R. and Esselink, P. (2013). Nature conservation 
of salt marshes: The influence of grazing on biodiversity. :32.

Vu, H.D., Więski, K. and Pennings, S.C. (2017). Ecosystem engineers drive creek formation in salt marshes. Ecology 98(1):162-174. doi: https://doi.org/10.1002/ecy.1628.

Vulliamy, B., Potts, S.G. and Willmer, P.G. (2006). The effects of cattle grazing on plantpollinator communities in a fragmented Mediterranean landscape. Oikos 114(3):529-543. doi: https://doi.org/10.1111/j.2006.0030-1299.14004.x.

van der Wal, R., van Lieshout, S.M.J. and Loonen, M.J.J.E. (2001). Herbivore impact on moss depth, soil temperature and arctic plant growth. Polar Biology 24:29-32. doi: https://doi.org/10.1007/s003000000170.

WallisDeVries, M.F., Bakker, J.P. and Van Wieren, S.E. (eds.) (1998). Grazing and Conservation Management. Dordrecht, The Netherlands: Kluwer Academic Publishers.

Wanner, A., Suchrow, S., Kiehl, K., Meyer, W., Pohlmann, N., Stock, M. and Jensen, K. (2014). Scale matters: Impact of management regime on plant species richness and vegetation type diversity in Wadden Sea salt marshes. Agriculture, Ecosystems \& Environment 182:69-79. doi: https://doi.org/10.1016/j.agee.2013.08.014.

Weis, J.S. and Butler, C.A. (2009). Salt Marshes: A Natural and Unnatural History. New Brunswick, NJ: Rutgers University Press.

Welsh Government (2013a). The Action Plan for Pollinators in Wales. Aberwystwyth.

Welsh Government (2013b). Glastir Commons 2014 Explanatory Booklet and How to Complete Guide. Available at: www.wales.gov.uk/glastir [Accessed: 2 February 2016].

Wichern, J., Wichern, F. and Joergensen, R.G. (2006). Impact of salinity on soil microbial communities and the decomposition of maize in acidic soils. Geoderma 137(1):100-108. doi: https://doi.org/10.1016/j.geoderma.2006.08.001.

Wickham, H. (2016). Ggplot2: Elegant Graphics for Data Analysis. New York: Springer-Verlag.

Wood, G.W., Mengak, M.T. and Murphy, M. (1987). Ecological importance of feral ungulates at Shackleford Banks, North Carolina. American Midland Naturalist 118(2):236-244.

Woodward, R.T. and Wui, Y.S. (2001). The economic value of wetland services: a metaanalysis. Ecological Economics 37(2):257-270. doi: https://doi.org/10.1016/s09218009(00)00276-7.

Wuellner, C.T. (1999). Nest site preference and success in a gregarious, ground-nesting bee Dieunomia triangulifera. Ecological Entomology 24(4):471-479. doi:

https://doi.org/10.1046/j.1365-2311.1999.00215.x.

Yando, E.S., Osland, M.J., Jones, S.F. and Hester, M.W. (2019). Jump-starting coastal wetland restoration: A comparison of marsh and mangrove foundation species. Restoration Ecology:e12963. doi: https://doi.org/10.1111/rec.12963.

Yu, O.T. and Chmura, G.L. (2010). Soil carbon may be maintained under grazing in a St Lawrence Estuary tidal marsh. Environmental Conservation 36(4):312-320. doi: https://doi.org/10.1017/s0376892910000184.

van Zanten, B.T., Verburg, P.H., Scholte, S.S.K. and Tieskens, K.F. (2016). Using choice modeling to map aesthetic values at a landscape scale: Lessons from a Dutch case study. Ecological Economics 130:221-231. doi: https://doi.org/10.1016/j.ecolecon.2016.07.008. 